Pacific Journal of Mathematics

ON A REMARKABLE CLASS OF POLYHEDRA IN COMPLEX 


\title{
ON A REMARKABLE CLASS OF POLYHEDRA IN COMPLEX HYPERBOLIC SPACE
}

\author{
G. D. Mostow
}

Dedicated to Gerhard Hochschild on the occasion of his 65th birthday

The Selberg, Piatetsky-Shapiro conjecture, now established by Margoulis, asserts that an irreducible lattice in a semi-simple group $G$ is arithmetic if the real rank of $G$ is greater than one. Arithmetic lattices are known to exist in the real-rank one group $\mathrm{SO}(n, 1)$, the motion group of real hyperbolic $n$-space, for $n \leqq 5$. These examples due to Makarov for $n=3$ and Vinberg for $n \leqq 5$ are defined by reflecting certain finite volume polyhedra in real hyperbolic $n$-space through their faces. The purpose of the present paper is to show that there are also nonarithmetic lattices in the real-rank one group $\mathrm{PU}(2,1)$, the group of motions of complex hyperbolic 2-space which can be defined algebraically and leads to remarkable polyhedra. This serves to help determine the limits of the Selberg, Piatetsky-Shapiro conjecture. The analysis of these polyhedra also leads to the first known example of a compact negatively curved Riemannian space which is not diffeomorphic to a locally symmetric space.

This paper arose out of an attempt to determine the limits of validity of the Selberg, Piatetsky-Shapiro conjecture on the arithmeticity of lattice subgroups. In $1960 \mathrm{~A}$. Selberg conjectured that apart from some exceptional $G$, an irreducible noncocompact lattice subgroup $\Gamma$ of a semi-simple group $G$ is arithmetic ("irreducible" in the sense that $\Gamma$ is not commensurable with a direct product of its intersections with factors of $G$ ). Later Piatetsky-Shapiro conjectured: An irreducible lattice of a semi-simple group $G$ is arithmetic if $R$-rank $G>1$.

The Selberg, Piatetsky-Shapiro conjecture was settled affirmatively by G. A. Margoulis in the striking paper that he submitted to the 1974 International Congress of Mathematicians in Vancouver.

The simple groups of $\boldsymbol{R}$-rank 1 are (up to a local isomorphism)

$$
\mathrm{SO}(n, 1), \mathrm{SU}(n, 1), \mathrm{SP}(n, 1), F_{4}
$$

which act as isometries on the hyperbolic space

$$
\boldsymbol{R} h^{n}, \boldsymbol{C h} h^{n} \boldsymbol{H} h^{n}, O h^{2}
$$

over the real numbers $\boldsymbol{R}$, the complex numbers $\boldsymbol{C}$, the quaternions (or Hamiltonians) $\boldsymbol{H}$, the octonians (or Cayley numbers) $\boldsymbol{O}$ respectively. Nonarithmetic lattices in $\mathrm{SO}(2,1)\left(=\mathrm{SL}_{2}(\boldsymbol{R}) / \pm 1\right)$ have been 
known for a long time. Nonarithmetic lattices in $\operatorname{SO}(n, 1)$ for $n=3$ where first found by V. S. Makarov (in 1965) and shortly thereafter E. B. Vinberg initiated an extensive investigation which turned up nonarithmetic lattices in $\mathrm{SO}(n, 1)$ for $n \leqq 5$. Both the Makarov and Vinberg examples are defined by reflecting a polyhedron $F$ of finite volume in $R h^{n}$ in its $(n-1)$-dimensional faces, which are pointwise fixed under the reflections. The $(n-1)$-dimensional faces thus must lie on geodesic subspaces of codimension ${ }_{R} 1$. The group $\Gamma$ generated by the reflections in the codimension- 1 faces of $F$ is a discrete group of isometries on $R h^{n}$ and has $F$ as a fundamental domain if and only if all the dihedral angles of $F$ are of the form $\pi$ integer.

Straightforward generalization of this method for finding lattice subgroups of isometries on the other hyperbolic spaces is blocked by the fact that only in $\boldsymbol{R}$-hyperbolic spaces do there exist codimension 1 geodesic subspaces. Thus it is not a priori clear what to take as the bounding surfaces of a polyhedron $F$ out of which we are to construct a group $\Gamma$ of isometries with $F$ as fundamental domain.

The principal result of this paper is the construction of a class of such polyhedra $F$ in $C h^{2}$. The guiding principle in the discovery of $F$ is the exploitation of symmetry. The polyhedron $F$ depends on two parameters, $(p, t)$ where $p=3,4,5$, and $|t|<3(1 / 2-1 / p)$. To each $F(p, t)$, there corresponds an infinite subgroup $\Gamma$ of $U(2,1)$ generated by three $\boldsymbol{C}$-reflections of order $p$. For some values of the parameter, the polyhedron $F$ is stabilized by a subgroup of $\Gamma$ of order 3. For only a finite number of values of the parameters is the group $\Gamma$ discrete. Whenever $\Gamma$ is discrete, it is a lattice subgroup.

The main theorems proved in this paper are:

TheOREM A (cf. §17.3). There exist in $\mathrm{PU}(2,1)$ nonarithmetic lattices generated by C-reflections of order 3,4, or 5 . Up to an isometry, any such lattice with Coxeter diagram

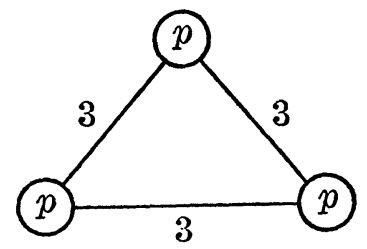

and phase shift $\varphi, \varphi^{3}=\exp \pi i t$, is given by the seven values

$$
\begin{aligned}
(p, t)= & (3,5 / 42),(3,1 / 12),(3,1 / 30) \\
& (4,3 / 20),(4,1 / 12) \\
& (5,1 / 5),(5,11 / 30)
\end{aligned}
$$


The noncocompact lattices $\Gamma(p, t)$ are arithmetic.

THEOREM (cf. §19). For every rational value of $t$, with $|t|<$ $1 / 2-1 / p$, there is a complex analytic manifold $Y(p, t)$ and a canonical $\Gamma(p, t)$ map $\pi: Y(p, t) \rightarrow C h^{2}$ such that $\Gamma(p, t)$ operates discontinuously and holomorphically on $Y(p, t)$.

The quotient of $Y$ by a torsion-free subgroup of $\Gamma$ leads to the first known example of a compact negatively curved Riemannian space $M$ which is not diffeomorphic to a locally symmetric space (cf. [8]). $M$ is in fact an algebraic surface and is negatively curved with respect to a Kaehler metric.

The major effort in proving Theorem A once the groups $\Gamma(p, t)$ have been defined, is to decide when $\Gamma(p, t)$ is discrete. Apart from $\S 4$ which gives a criterion for the arithmeticity of a lattice, most of $\S 3$ to $\S 17$ is aimed at the discreteness question. The choice of the complex analytic structure in Theorem $B$ with respect to which $\Gamma$ operates discontinuously and holomorphically depends on the results in $\S 6$ (though not on $\S 6.5$ ) and $\S 18$. In particular, explicit information (cf. $\$ 18.2$ ) about the star of each vertex in the polyhedral space $Y$ is exploited.

In anticipation of generalizing the construction given here to $n=3$ and 4 , the results in $\S 3$ on spinal surfaces are presented for general $n$.

Part of the results contained in this paper were announced in [7].

A large part of this paper is devoted to computations. At an early stage of the investigation of discreteness, I profited greatly from computer exploration. Without the computer it would have been very difficult to recognize how much more complicated is the fundamental domain of the group of order 72 generated by two $C$-reflections with Coxeter diagram

$$
\text { (3) } \frac{4}{-3}
$$

than the fundamental domain for the group of order $24(p / 6-p)^{2}$ generated by two $\boldsymbol{C}$-reflections with Coxeter diagram

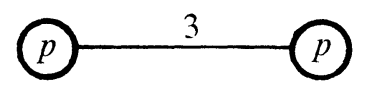

Thus the discovery of the family $F(p, t)$ owes much to the vastly wider exploration which the computer permits. Once the target began to be discerned, it became possible to verify all the remarkable properties of the family $F(p, t)$ by a combination of geometric 
and algebraic methods. The account here is in the strictly logical (rather than psychological) sense totally independent of the computer.

I take pleasure in acknowledging my debt and gratitude to my associate and students at Yale who programmed the algorithms described in $\S 7,8$ and other related capabilities. In chronological order of their assistance, I was enormously helped by Dr. Sidnie Feit, Alex Feingold, James Cogdell, Daniel Bar-Yacov, and Edna Bar-Yacov.

\section{Algebraic preliminaries.}

\subsection{C-reflections.}

Let $V$ be an $n$-dimensional vector space over $C$. A $C$-reflection in $V$ is a linear map $R: V \rightarrow V$ of finite order with $n-1$ eigenvalues equal to 1 . A $C$-reflection can be expressed in the form $x \rightarrow x+\beta(x) e$ where $\beta$ is a linear function and $1+\beta(e)$ is a root of unity other than 1 .

Let $H$ be a nondegenerate hermitian form on $V$ and denote $H(v, w)$ by $\langle v, w\rangle$ for all $v, w \in V$. Given $e \in V$ with $\langle e, e\rangle=1$ and a positive integer $p$, we denote by $R_{e, p}$ the $C$-reflection

$$
x \longrightarrow x+(\xi-1)\langle x, e\rangle e, \quad \xi=\exp 2 \pi \sqrt{-1} / p .
$$

$R_{e, p}$ fixes each point in $e^{\perp}=\{x \in V ;\langle x, e\rangle=0\}$ and has order $p$. Clearly $R_{e, p}$ preserves $H$.

Suppose $\left\{e_{1}, \cdots, e_{n}\right\}$ is a linearly independent set of unit vectors and let $R_{i}=R_{e_{i}, p_{i}}$. Set $\Gamma=\left\{\left\{R_{1}, R_{2}, \cdots, R_{n}\right\}\right\}$, the group generated by the set $\left\{R_{1}, \cdots, R_{n}\right\}$. Then $\Gamma \subset U(H)$, the unitary group of the hermitian form $H$.

The hermitian form $H$ need not be positive definite. However if $\Gamma$ is finite and irreducible, then $H$ must be positive definite. For by Schur's lemma, an irreducible group stabilizes a unique hermitian form up to scalar factor; on the other hand, a finite group preserves a definite hermitian form. Since $H\left(e_{i}, e_{i}\right)=1, H$ is positive definite if $\Gamma$ is irreducible. We will see in $\S 2.3$ how to drop this last hypothesis.

\subsection{Finite groups generated by two C-reflections.}

Let $\left\{e_{1}, e_{2}\right\}$ be a base of the $C$-vector space $V$ and let $H$ be a hermitian form on $V$. The condition that $\left\{R_{e_{1}, p_{1}}, R_{e_{2}, p_{2}}\right\}$ generate a finite group $\Gamma$ may be determined as follows.

Consider the 1 dimensional projective space $C P^{1}$ of one-dimensional subspaces of $V$; let $\pi: V-\{0\} \rightarrow C P^{1}$ denote the canonical 
projection. Let $R_{i}$ denote the action of $R_{e_{1}, p_{1}}$ on $C P^{1}$, and denote $\pi\left(e_{i}^{\frac{1}{i}}\right)$ by $* e_{i}^{\frac{1}{2}}(i=1,2)$.

$C P^{1}$ may be identified with the standard 2-sphere $S^{2}$, and $\Gamma$ operates on $S^{2}$ by rotations. Thus $\Gamma$ is finite if and only if the group $\left\{R_{1}, R_{2}\right\}$ generated by rotations of angle $2 \pi / p_{i}$ at ${ }^{*} e_{i}^{\frac{1}{i}}$ is finite. This is the case if and only if there is a $\gamma$ in $\Gamma$ such that the geodesic triwngle with base $\gamma\left({ }^{*} e_{1}^{\frac{1}{1}}\right)^{*} e_{2}^{\perp}$ and base angles $\pi / p_{1}, \pi / p_{2}$ has as third angle $2 \pi / q$ where

$$
\frac{1}{p_{1}}+\frac{1}{p_{2}}+\frac{2}{q} \geqq 1 \text { and } q \text { is even if } p_{1} \neq p_{2} .
$$

From spherical trigonometry the length of the base ${ }^{*} e_{1}^{L *} e_{2}^{\perp}$ in a geodesic triangle with angles $\pi / p_{1}, \pi / p_{2}, 2 \pi / q$ is given by

$$
\cos d\left({ }^{*} e_{1}^{\perp},{ }^{*} e_{2}^{\perp-1}\right)=\frac{\cos \pi / p_{1} \cdot \cos \pi / p_{2}+\cos 2 \pi / q}{\sin \pi / p_{1} \cdot \sin \pi / p_{2}} .
$$

On the other hand, the inner-product on $V$ is related to the metric on $S^{2}$ by the formulae

$$
\left|\left\langle e_{1}, e_{2}\right\rangle\right|=\cos \sigma, d\left({ }^{*} e_{1}^{\perp},{ }^{*} e_{2}^{\llcorner}\right)=2 \sigma .
$$

Therefore, by the half-angle formula

$$
\left|\left\langle e_{1}, e_{2}\right\rangle\right|=\left(\frac{\cos \left(\pi / p_{1}-\pi / p_{2}\right)+\cos 2 \pi / q}{2 \sin \pi / p_{1} \cdot \sin \pi / p_{2}}\right)^{1 / 2} .
$$

Let $\varphi$ be any complex number with $|\varphi|=1$ and let $H(\varphi)$ denote the hermitian form on $C^{2}$ given by

$$
\begin{aligned}
& \left\langle e_{i}, e_{i}\right\rangle=1, \quad(i=1,2) \\
& \left\langle e_{1}, e_{2}\right\rangle=\alpha \varphi
\end{aligned}
$$

where $\alpha$ is given by (2.2.2). Let $\Delta(\varphi)$ denote the determinant of the matrix $\left(\left\langle e_{i}, e_{j}\right\rangle\right)$. Then

$$
\Delta(\varphi)=1-\alpha^{2}=\sin ^{2} \sigma>0
$$

if $H$ is nondegenerate. We note that $\Delta(\varphi)$ is independent of $\varphi$. Let $\Gamma(\varphi)$ denote the group $\left\{\left\{R_{e_{1}, p_{1}}, R_{e_{2}, p_{2}}\right\}\right\}$ corresponding to $H_{\varphi}$. Replacing $e_{1}$ by $e_{1} \phi$ does not change $R_{e_{1}, p_{1}}$ and provides an isometry of $H(1)$ to $H(\varphi)$. Thus $\Gamma(\varphi)$ is independent of $\varphi$.

Set $R_{i}=R_{e_{i}, p_{i}}(i=1,2)$. Suppose that $H$ satisfies (2.2.1) and (2.2.2). Then the group $\Gamma=\left\{\left\{R_{1}, R_{2}\right\}\right\}$ has the relations

$$
\begin{gathered}
R_{1}^{p_{1}}=1,\left(R_{2}\right)^{p_{2}} \\
\left(R_{1} R_{2}\right)^{g / 2}=\left(R_{2} R_{1}\right)^{q^{\prime / 2}} .
\end{gathered}
$$

If $q$ is odd, the second relation signifies 


$$
R_{1} R_{2} \cdots R_{1}=R_{2} R_{1} \cdots R_{2}
$$

(cf. [4]). Moreover, the center of the group $\Gamma$ is cyclic and generated by

$$
\left\{\begin{array}{l}
\left(R_{1} R_{2}\right)^{q} \text { if } q \text { is odd } \\
\left(R_{1} R_{2}\right)^{q / 2} \text { if } q \text { is even }
\end{array}\right.
$$

(this corresponds to the fact that on $S^{2}, R_{1} R_{2}$ rotates about the vertex $e_{0}$ opposite ${ }^{*} e_{1}^{\perp *} e_{2}^{\perp}$ through an angle of $\left.4 \pi / q\right)$.

Let $s$ denote the reciprocal of the spherical excess of the geodesic triangle $e_{0}^{*} e_{1}^{*} e_{2}^{\perp}$ i.e., $s^{-1}=p_{1}^{-1}+p_{2}^{-1}+2 q^{-1}-1$ then the order of the center is (cf. [4])

$$
\left\{\begin{array}{l}
\frac{2 s}{q}, q \text { odd } \\
\frac{4 s}{q}, q \text { even }
\end{array}\right.
$$

and the order of $\Gamma$ is given by

$$
\# \Gamma=\frac{8 s^{2}}{q} .
$$

\subsection{Coxeter diagrams, phase shifts.}

To each finite group generated by $\boldsymbol{C}$-reflections, Coxeter has associated a diagram. In the case of a group generated by two reflections $\left\{R_{e_{1}, p_{1}}, R_{e_{2}, p_{2}}\right\}$ with

$$
\left\langle e_{1}, e_{2}\right\rangle=\alpha \varphi
$$

with $\alpha$ given by (2.2.2) and (2.2.1) satisfied, the Coxeter diagram consists of two nodes and a line

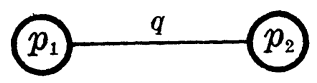

if $q>2$; if $q=2$, no line is drawn joining the nodes. This diagram determines $\left\{R_{e_{1}, p_{1}}, R_{e_{2}, p_{2}}\right\}$ uniquely up to an isomorphism.

To a group generated by $n$-reflections every two of which generate a finite group, one associates a diagram made up of $n$ nodes, attaching $p_{i}$ the order of the reflection $R_{i}$ to the $i$ th node; the nodes $i, j$ are joined by a line labeled $q_{i j}$ if $R_{i}=R_{e_{i}, p_{i}}, R_{j}=$ $R_{e_{j}, p_{j}}$ and the $q_{i j}$ (resp. $q_{i j} / 2$ ) is the lowest power $p$ of $R_{i} R_{j}$ which is in the center of $\Gamma_{i j}=\left\{\left\{R_{i}, R_{j}\right\}\right\}$ if $p$ is odd (resp. even). Conversely, to any diagram $\mathscr{D}$

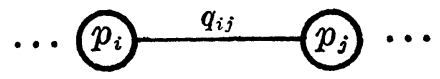

with $n$ nodes, satisfying (2.2.1), one associates a family of groups 
$I$ generated by $n$-reflections as follows:

In $C^{n}$, let $e_{1}, e_{2}, \cdots, e_{n}$ be the standard base. Define the hermitian form $H$ :

$$
\begin{aligned}
& \left\langle e_{i}, e_{j}\right\rangle=-\alpha_{i j} \varphi_{i j} \quad i \neq j \\
& \left\langle e_{i}, e_{i}\right\rangle=1
\end{aligned}
$$

where

$$
\alpha_{i j}=\left(\frac{\cos \left(\pi / p_{1}-\pi / p_{2}\right)+\cos 2 \pi / q_{i j}}{2 \sin \pi / p_{1} \cdot \sin \pi / p_{2}}\right)^{1 / 2},\left|\varphi_{i j}\right|=1
$$

and define $R_{i}=R_{e_{i}, p_{i}}(i=1, \cdots, n)$. For any $i, j$, the group $\Gamma_{i j}=$ $\left\{\left\{R_{i}, R_{j}\right\}\right\}$ is finite and independent of the choice of $\varphi_{i j}$, up to an isomorphism. More generally, if the graph of $\mathscr{D}$ is a tree, then one can replace $e_{i}$ by $\varphi_{i} e_{i}(i=1, \cdots, n)\left|\varphi_{i}\right|=1$, so as to get an isomorphism between any two groups $\Gamma$ in the above family. For any loop $i_{1} i_{2} \cdots i_{k}$ in the graph, the product $\varphi_{i, i_{2}} \cdot \varphi_{i_{2} i_{2}} \cdots \varphi_{i_{k} i_{1}}$ is invariant under such changes, and indeed two groups $\Gamma$ with the same diagram need not be isomorphic. The set $\left\{\varphi_{i j} ; i \neq j, i, j=\right.$ $1, \cdots, n\}$ is called the phase shift of the hermitian form $H$. Two phase shifts define isomorphic groups $\Gamma$ if the products of phase shifts over all closed loops are equal. A phase shift is called rational if it is a root of unity.

The data ( $\mathscr{D}$, phase shifts) determines a unique hermitian form $H$ and a unique group $\Gamma$ generated by $C$-reflections; we sometimes denote $\Gamma$ by $\Gamma(\mathscr{D}, H)$.

(2.3.3) If the Coxeter diagram of a group $\Gamma$ is connected and the hermitian form $H$ is nondegenerate, then the group operates irreducibly on the underlying vector space.

The proof is essentially the same as for Coxeter groups of $\boldsymbol{R}$ -

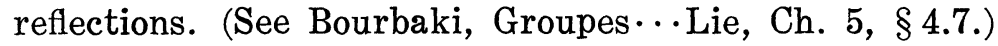

Unlike the case of $\boldsymbol{R}$-reflection groups, groups with different Coxeter diagrams may be isomorphic.

As a consequence of (2.3.3), if the hermitian form $H$ of the data, $(\mathscr{D}, H)$ is nondegenerate and the group $\Gamma$ is finite, then $H$ is positive definite. For $\Gamma$ is a direct sum of irreducible groups corresponding to the connected components on the diagram $\mathscr{D}$.

On the summand corresponding to each component, $H$ is positive definite by the result in $\S 2.1$. Hence $H$ is positive definite.

\subsection{Finite groups generated by $C$-reflections.}

Finite subgroups of $\operatorname{PGL}(n, \boldsymbol{C})$ generated by $\boldsymbol{C}$-reflections were 
classified near the turn of the century (cf. G. Bagnera, I gruppi finiti di tranzformazioni lineari dello spazio che contegono omologie, Rend. Circ. Mat. Palermo, 19 (1905), pp. 1-56; $C$-reflections on projective space were named homologies in those days). Subsequently the classification was redone by G. L. Shephard and J. A. Todd in 1953 (see [10]) and then by Coxeter in 1966 (see [4]). Coxeter has listed the group diagrams.

Finite linear groups $\Gamma$ in $n$-variables generated by $C$-reflections are characterized by the property:

$\Gamma$ possesses a set of $n$ algebraically independent invariant forms $I_{1}, I_{2}, \cdots, I_{n}$ of degrees $m_{1}+1, m_{2}+1, \cdots, m_{n}+1$ such that

$$
\prod_{i=1}^{n}\left(m_{i}+1\right)=\# \Gamma
$$

(see [10] or (Bourbaki, Groupes $\cdots$ Lie, Ch. V §5.5) for this and other properties).

If $\Gamma$ is a finite linear group generated by $\boldsymbol{C}$-reflections (2.4.2) $\quad\left\{R_{1}, \cdots, R_{n}\right\}$, then any $C$-reflection $R \in \Gamma$ is conjugate to a power of some $R_{i}(i=1, \cdots, n)$ : (See [3], Lemma 4.11 (iii).)

Given complex reflections $R_{e_{1}, p_{1}}$ and $R_{e_{2}, p_{2}}$ on a vector space $V$, they generate a group $\Gamma^{\prime}$ which stabilizes $W=C e_{1}+C e_{2}$ and fixes each point of $e_{1}^{\perp} \cap e_{2}^{\perp}$. If

$$
\operatorname{det}\left(\begin{array}{cc}
\left\langle e_{1}, e_{1}\right\rangle & \left\langle e_{1}, e_{2}\right\rangle \\
\left\langle e_{2}, e_{1}\right\rangle & \left\langle e_{2}, e_{2}\right\rangle
\end{array}\right)=1-\left|\left\langle e_{1}, e_{2}\right\rangle\right|^{2}>0
$$

the restriction of the hermitian form $H$ to $W$ is positive definite. Hence $\Gamma^{\prime}$ is faithfully represented in the compact unitary group of the restriction of $H$ to $W$. Thus $\Gamma^{\prime}$ is discrete if and only if it is finite.

One can use this observation to determine that some groups are not discrete. For this purpose, one needs a list of all possible admissible values of $\left|\left\langle e_{1}, e_{2}\right\rangle\right|$ if $R_{e_{1}, p_{1}}$ and $R_{e_{2}, p_{2}}$ are to lie in a compact group and be discrete. Such reflections lie in groups $\Gamma$ generated by two $C$-reflections, such that $\left|\left\langle e_{1}, e_{2}\right\rangle\right|$ is given by the number $\alpha$ on the right hand side of (2.2.2). For a finite linear group $\Gamma$ generated by $\boldsymbol{C}$-reflections, any reflection is conjugate to a power of a generator (cf. (2.4.2)). Thus the admissible values can be read off from the list of $\left\{\left|\left\langle e_{1}, \gamma e_{2}\right\rangle\right| ; \gamma \in \Gamma\right\}$ corresponding to Coxeter diagrams with two nodes. We list only the connected diagrams with $p_{1}=p_{2}$ and values of $\left|\left\langle e_{1}, \gamma e_{2}\right\rangle\right| \neq 0,1$. 

$\mathscr{D}$
$\left|\left\langle e_{1}, \gamma e_{2}\right\rangle\right|$
(2) $\frac{q}{(2)}$
$\cos k \pi \mid q$
(3) 3
.577
(4) $\frac{3}{-(4)}$
.707
(5) 3
$.851, .526$
(3) $\frac{4}{5}$
$.816, .577$
(3) 5
$.934, .356, .951, .309$

(2.4.3)

\subsection{Lattice subgroups.}

Let $G$ be a locally compact group. A lattice $\Gamma$ in $G$ is a discrete subgroup such that $G / \Gamma$ has finite Haar measure. Two subgroups $\Gamma$ and $\Gamma^{\prime}$ of $G$ are called commensurable if $\Gamma \cap \Gamma^{\prime}$ is of finite index in both $\Gamma$ and $\Gamma^{\prime}$.

If $G$ is a semi-simple matrix group having no compact normal subgroup of positive dimension and $\Gamma$ is a lattice in $G$, then $\Gamma$ is dense in the Zariski-topology of $G$; that is, any polynomial in the matrix entries of $G$ which vanishes on $\Gamma$ vanishes on $G$ ([1], cf. [9]). In particular, if $G$ acts irreducibly on the underlying vector space, so does $\Gamma$. This last remark holds equally well if $G$ is reductive (i.e., $G$ is a product of a semi-simple group and a commuting abelian subgroup), and $G$ acts (absolutely) irreducibly on the underlying complex vector space.

If $G$ is a Lie group, we denote by $\mathrm{Ad} G$ the representation of $G$ on its Lie algebra $G$ induced by $x \rightarrow g x g^{-1}$.

Suppose now that $G$ is a reductive Lie group of matrices and $\Gamma$ a lattice subgroup such that Ad $\Gamma$ is Zariski-dense in Ad $G$. Let $k$ denote the field generated over the field $\boldsymbol{Q}$ of rational numbers by $\operatorname{Tr} \operatorname{Ad} \Gamma=\{\operatorname{Tr} \operatorname{Ad} \gamma ; \gamma \in \Gamma\}$. Let $T$ denote the function $g \rightarrow \operatorname{Tr} \operatorname{Ad} g$ on $G$. Let $\mathscr{L}$ denote the $\boldsymbol{C}$-linear span of the functions

$$
x \longrightarrow T(x \gamma), \quad x \in G, \gamma \in \Gamma \text {. }
$$

The group $G$ acts on the space $\mathscr{F}$ of all. polynomial functions on $G$ via the translations:

$$
f \longrightarrow g \cdot f, \quad f \in \mathscr{F}, g \in G
$$

where we define $(g \cdot f)(x)=f(x g)$. Clearly $\mathscr{L} \subset \mathscr{F}$ and $\Gamma \cdot \mathscr{L} \subset \mathscr{L}$. Since $T(x \gamma)=\operatorname{Tr}(\operatorname{Ad} x \gamma)=\operatorname{Tr}(\operatorname{Ad} x \operatorname{Ad} \gamma)$ and $\operatorname{Ad} \Gamma$ is Zariski-dense in $G$, it follows that $G \cdot \mathscr{L} \subset \mathscr{L}$. The set of functions $\{\gamma \cdot T ; \gamma \in \Gamma\}$ span $\mathscr{L}$ and from among them we select a base $B=\left\{\gamma_{1} \cdot T, \cdots, \gamma_{n} \cdot T\right\}$ (note: $n \leqq(\operatorname{dim} G)^{2}$ and is therefore finite). 
In the representation $\rho$ of $G$ on $\mathscr{L}$, the matrix of $\rho(\gamma)$ with respect to the base $B$ is k-valued.

Proof. (cf. [5]). For any $c \in G$, let $\hat{c}$ denote the evaluation map $\mathscr{F} \rightarrow C$ given by $f \rightarrow f(c) . \quad \hat{c}$ is linear. Since Ad $\Gamma$ is Zariski dense in $\operatorname{Ad} G,\{\hat{\gamma} ; \gamma \in \Gamma\}$ is a separating family of linear functions on $\mathscr{L}$. Hence we can select $\beta_{1}, \cdots, \beta_{n}$ in $\Gamma$ so that $\left\{\widehat{\beta}_{1}, \cdots, \widehat{\beta}_{n}\right\}$ separates $\mathscr{L}$. For any $\gamma \in \Gamma$ and for fixed $i$,

$$
\begin{aligned}
& \widehat{\beta}_{k}\left(\gamma \cdot \gamma_{i} T\right)=\widehat{\beta}_{k}\left(\sum_{j} c_{i j} \gamma_{j} \cdot T\right) \\
& T\left(\beta_{k} \gamma \gamma_{i}\right)=\sum_{j=1} c_{i j} T\left(\beta_{k} \gamma_{j}\right) \quad k=1, \cdots, n .
\end{aligned}
$$

This gives a system of $n$ equations for the $n$ unknowns $c_{i 1}, \cdots$, $c_{i n}$, with all coefficients in $k$. Hence the solutions are in $k$. Since $\rho(\gamma)=\left(c_{i j}\right)$, our assertion is proved.

The representation $\rho$ provides a faithful representation of $\mathrm{Ad} G$. The Zariski-closure $G^{*}$ of $\rho(G)$ in the full $n^{2} \times n^{2}$ matrix algebra coincides with the Zariski-closure of $\rho(\Gamma)$. Let $I$ denote the ideal of polynomial functions on $\operatorname{Hom}_{c}(\mathscr{L}, \mathscr{L})$ which vanish on $G^{*}$. Since $\rho(\Gamma)$ is Zariski-dense in $G^{*}$ and the matrix entries of $\rho(\Gamma)$ are in $k$, the ideal $I$ has a base of polynomial functions with coefficients in $k$. Thus (strictly by definition),

(2.5.1) The algebraic group $G^{*}$ is defined over the field $k$.

\section{Geometric preliminaries.}

\subsection{The ball $B$ and its isometries.}

Let $V$ be an $n+1$-dimensional vector space over $C$ on which we fix a hermitian form $H$ of signature $(n, 1)$ i.e., $n$ plus signs and 1 minus sign. The unitary group of $H$ operates on the projective space $\boldsymbol{P}_{n}$ of one-dimensional $\boldsymbol{C}$-subspaces of $V$; we denote the resulting group on $\boldsymbol{P}_{n}$ by $P U(H)$. Set $\langle p, q\rangle=H(p, q)$ and

$$
\begin{aligned}
& V^{-}=\{p \in V ;\langle p, p\rangle\langle 0\} \\
& V^{0}=\{p \in V ;\langle p, p\rangle=0\} \\
& { }^{\circ} V^{+}=\{p \in V ;\langle p, p\rangle>0\} .
\end{aligned}
$$

Let $\pi$ denote the canonical map of $V-\{\{0\}\}$ onto $\boldsymbol{P}_{n}$. Set

$$
B=\pi\left(V^{-}\right) \text {, Aut } B=\text { the restriction of } P U(H) \text { to } B \text {. }
$$

On $B$ one can define a Riemannian positive definite infinitesimal metric invariant under Aut $B$ by the formula (cf. [6])

$$
d s^{2}=-\frac{1}{\langle p, p\rangle^{2}}\left|\begin{array}{ll}
\langle d p, d p\rangle & \langle d p, p\rangle \\
\langle p, d p\rangle & \langle p, p\rangle
\end{array}\right| \text {. }
$$


Although there are many choices for $p$ which map to the same $\pi(p)$, the formula above yields a well-defined metric. The angle between two tangent vectors to $B$ at a point $\pi(p)$ is defined by the usual associated real-valued inner product formula

$$
(x, y)=-\operatorname{Re} \frac{1}{\langle p, p\rangle^{2}}\left|\begin{array}{ll}
\langle x, y\rangle & \langle x, p\rangle \\
\langle p, y\rangle & \langle p, p\rangle
\end{array}\right|,
$$

thus

$$
\cos \Varangle(x, y)=\frac{(x, y)}{[(x, x)(y, y)]^{1 / 2}}
$$

In particular, if $x$ and $y$ are nonzero tangent vectors to $B$ at a point $\pi(p)$ with $y=\alpha x, \alpha \in C$, then $\Varangle(x, y)=\arg \alpha$.

The distance between two points $\pi(p)$ and $\pi(q)$ in $B$ is given by the formula (cf. [6])

$$
\cosh d(\pi(p), \pi(q))=\frac{|\langle p, q\rangle|}{(\langle p, p\rangle\langle q, q\rangle)^{1 / 2}} .
$$

For any vector $C$-subspace $W$ of $V$ with $\operatorname{dim}_{c} W=k+1$ on which the signature of the restriction of $H$ to $W$ is $(k, 1)$, the intersection $W \cap V^{-}$is nonempty. $\pi\left(W \cap V^{-}\right)$is called a $C$-k-plane in $B$; and $W$ is called its preimage in $V$. In case $k=1, \pi\left(W \cap V^{-}\right)$is called a $C$-line in $B$. A $C$ - $k$-plane is clearly a geodesic subspace of $B$ for $k=0,1, \cdots, n$.

By the principal axis theorem, there is a base of $C$-linear functions on $V$ such that

$$
H=-\left|x_{0}\right|^{2}+\left|x_{1}\right|^{2}+\cdots+\left|x_{n}\right|^{2} .
$$

Such a base $\beta$ is called a standardizing coordinate system; it is not unique, any other differing from it by a unitary transformation of $H$. The associated nonhomogeneous coordinate system $\left\{x_{1} / x_{0}, \cdots\right.$, $\left.x_{n} / x_{0}\right\}$ on the complement of $x_{0}=0$ in $\boldsymbol{P}_{n}$ is called a standard nonhomogeneous system. In the nonhomogeneous coordinates $x_{1} / x_{0}, \cdots$, $x_{n} / x_{0}$ of $\beta, B$ becomes the open unit ball in $C^{n}$ :

$$
\left(\frac{x_{1}}{x_{0}}\right)^{2}+\cdots+\left(\frac{x_{n}}{x_{0}}\right)^{2}<1 .
$$

The point $o$ of $B$ defined by the $n$ equations

$$
0=x_{1}=x_{2}=\cdots=x_{n}
$$

is called the center of the standardizing and of the standard nonhomogeneous coordinates. Every point of $B$ is the center of some 
standardizing $\beta$.

The stabilizer (Aut $B)_{0}$ of the center $o$ of $\beta$ is the image in Aut $B$ of the subgroup of $\mathrm{PU}(H)$ given in any standardizing coordinates by

$$
\begin{gathered}
x_{0}^{\prime}=\alpha x_{0} \\
\left(\begin{array}{c}
x_{1}^{\prime} \\
\vdots \\
x_{n}
\end{array}\right)=U\left(\begin{array}{c}
x_{1} \\
\vdots \\
x_{n}
\end{array}\right)
\end{gathered}
$$

where ${ }^{t} \bar{U}=U^{-1}$. The group $U(H)$ is transitive on $\pi\left(V^{-}\right)$and the subgroup (Aut $B)_{o}$ is transitive on the set of all $C$-lines through $o$. It follows at once that Aut $B$ is transitive on all pointed lines i.e., pairs $(p, L)$ with $p$ a point on the $C$-line $L$ of $B$.

The geodesic subspaces of $B$ having constant negative curvature are of two distinct kinds. On the one hand by a direct computation one finds that all $C$-lines have constant curvature -2 . On the other hand, given in $V$ any $\boldsymbol{C}$-linearly independent vectors $f_{0}, \cdots, f_{k}$ such that $\left\langle f_{i}, f_{j}\right\rangle \in \boldsymbol{R}(i, j=0, \cdots, k)$, the subspace $\pi\left(\left[\boldsymbol{R} f_{0}+\cdots+\right.\right.$ $\left.\left.\boldsymbol{R} f_{k}\right] \cap V^{-}\right)$is called an $\boldsymbol{R}$-k-plane if it is nonempty; its sectional curvature is $-1 / 2$. If $k=1$, we call the $R$-1-plane an $R$-line.

REMARK 1. The ratio of 4 between the curvatures of $\boldsymbol{C}$-lines and $\boldsymbol{R}$-planes arises from the existence of a restricted $\boldsymbol{R}$-root $2 \alpha$. Any $C$-line is isometric to real hyperbolic 2-space i.e., the Poincare disc.

REMARK 2. With respect to any nonhomogeneous coordinate system on $B$, a $C$ - $k$-plane is the intersection with $B$ of a $k$-plane; or as we shall say for short, a $C$-k-plane is linear with respect to any nonhomogeneous coordinate system. By contrast, an $\boldsymbol{R}$ - $k$-plane $\pi\left(\left[\boldsymbol{R} f_{0}+\cdots+\boldsymbol{R} f_{k}\right] \cap V^{-}\right)$is the intersection with $B$ of a $k$-dimensional $\boldsymbol{R}$-linear subspace of $\boldsymbol{C}^{n}$ relative to nonhomogeneous coordinates $x_{1} / x_{0}, \cdots, x_{n} / x_{0}$ provided that $f_{0}, \cdots, f_{k}$ lies in the $\boldsymbol{R}$-linear span of the dual base to $x_{0}, \cdots, x_{n}$. Thus, with respect to a standard nonhomogeneous coordinate system, an $\boldsymbol{R}$ - $k$-plane need not be linear; for example, a general $\boldsymbol{R}$-line is a circular arc meeting the boundary of $A$ orthogonally, where $A$ is the unique $C$-line containing it.

Remark 3. Any geodesic line $P$ in $B$ is an $R$-line. For let $p_{0}, p_{1}$ be vectors in $V^{-}$such that $\pi\left(p_{0}\right)$ and $\pi\left(p_{1}\right)$ are distinct points of $P$. Set $M=C p_{0}+C p_{1}$ and $L=\pi\left(M \cap V^{-}\right)$. Then $L$ is the unique $C$-line containing $P$. Set $e_{0}=\left\langle p_{0}, p_{0}\right\rangle^{-1} p_{0}$ and $e_{1} \in M \cap\left(C e_{0}\right)^{\perp}$ where $\perp$ denotes the orthogonal subspace of $V$ with respect to $H$. Then 
$P=\pi\left(\left(e_{0}+\boldsymbol{R} e_{1}\right) \cap V^{-}\right)$and is thus an $\boldsymbol{R}$-line. We note that $\left\langle p_{0}, p_{1}\right\rangle \neq$ 0 since the restriction of the hermitian form $H$ to $M$ is of signature $(1,1)$. Since $\pi\left(p_{0}\right)=\pi\left(\left\langle p_{0}, p_{1}\right\rangle^{-1} p_{0}\right)$, we find that $P=\pi\left(\left\langle p_{0}, p_{1}\right\rangle^{-1} p_{0}+\right.$ $\left.\boldsymbol{R} p_{1}\right)$ is the geodesic line through $\pi\left(p_{0}\right)$ and $\pi\left(p_{1}\right)$.

One can say that with respect to a standard nonhomogeneous coordinate $z$ on a $\boldsymbol{C}$-line, an $\boldsymbol{R}$-line is linear with respect to $\operatorname{Re} z$ and $\operatorname{Im} z$ if and only if it passes through the origin.

REMARK 4. Inasmuch as $U(n)$ is transitive on all points of $\left\{z \in C^{n} ;|z|=1\right\}$, it follows that Aut $B$ is transitive on all pairs $(p, P)$ with $p \in B$ and $P$ an $R$-line through $p$.

\subsection{Properties of an equidistant surface.}

Given two points $\pi\left(p_{1}\right)$ and $\pi\left(p_{2}\right)$ in $B$, the equidistant surface $S$ of $\pi\left(p_{1}\right), \pi\left(p_{2}\right)$ is by definition

$$
S=\left\{\pi(x) \in B ; d\left(\pi(x), \pi\left(p_{1}\right)\right)=d\left(\pi(x), \pi\left(p_{2}\right)\right)\right\} .
$$

The equation of $S$ is easily obtained:

$$
\begin{aligned}
& \cosh d\left(\pi(x), \pi\left(p_{1}\right)\right)=\cosh d\left(\pi(x), \pi\left(p_{2}\right)\right) \\
& \frac{\left|\left\langle x, p_{1}\right\rangle\right|}{\left(\langle x, x\rangle\left\langle p_{1}, p_{1}\right\rangle\right)^{1 / 2}}=\frac{\left|\left\langle x, p_{2}\right\rangle\right|}{\left(\langle x, x\rangle\left\langle p_{2}, p_{2}\right\rangle\right)^{1 / 2}} .
\end{aligned}
$$

If moreover we normalize the points of $V$ representing $\pi\left(p_{1}\right)$ and $\pi\left(p_{2}\right)$ so that $\left\langle p_{1}, p_{1}\right\rangle=\left\langle p_{2}, p_{2}\right\rangle$, the equation of $S$ becomes simply

$$
\left|\left\langle x, p_{1}\right\rangle\right|=\left|\left\langle x, p_{2}\right\rangle\right| \text {. }
$$

The locus of this equation is easy to describe. Let $M$ denote the 2 dimensional $\boldsymbol{C}$-linear subspace $\boldsymbol{C} p_{1}+\boldsymbol{C} p_{2}$ of $V$. Let $A$ denote the orthogonal complement to $M$ in $V$ with respect to $H$. We have $A \cap M=(0)$ since the restriction of $H$ to $M$ has signature $(1,1)$ and thus $H$ on $A$ must be positive definite. $\pi\left(M \cap V^{-}\right)$is a $C$-line in $B$ and $P=S \cap \pi\left(M \cap V^{-}\right)$is the perpendicular bisector in the Poincare disc $\pi\left(M \cap V^{-}\right)$of the points $\pi\left(p_{1}\right)$ and $\pi\left(p_{2}\right)$. Now for any $x$ in $V$, if $x$ satisfies $\left|\left\langle x, p_{1}\right\rangle\right|=\left|\left\langle x, p_{2}\right\rangle\right|$ so also do the points $x+A$. Hence $S$ contains $\bigcup_{\pi(x) \in P} \pi(C x+A) \cap B$.

Let $x_{0}, \cdots, x_{n}$ be standardizing coordinates with $x_{2}=x_{3}=\cdots=$ $x_{n}=0$ on $M$ and $x_{0}=x_{1}=0$ on $A$. With respect to nonhomogeneous coordinates $x_{1} / x_{0}, \cdots, x_{n} / x_{0}$ on $C^{n}$ each of the planes $\pi(C x+A)$ is parallel to $\pi(\boldsymbol{C} y+\boldsymbol{A})$ (i.e., they meet only in the line at infinity $x_{0}=0$ ). Thus we see that $S$ contains the $R$ - $(2 n-1)$-dimensional cylinder erected on the geodesic line $P$.

Consider now the orthogonal projection $\mu$ (with respect to the 
Riemannian metric) of $B$ onto the geodesic $C$-line $\pi\left(M \cap V^{-}\right)$. If $\pi(x) \in S$ it is easy to verify that in the Riemannian metric the $C$ $(n-1)$-plane $\pi(C x+A) \cap B$ is orthogonal to the $C$-line $\pi(M) \cap B$. Hence $\mu(\pi(x)) \in P$. Thus $\pi(C x+A) \cap B=\mu^{-1} \mu(\pi(x))$ for all $x \in S$,

$$
S=\bigcup_{p \in P} \mu^{-1}(p)
$$

and $S$ is indeed the $(2 n-1)$-dimensional cylinder elected on $P$ with respect to both the Riemannian metric as well as nonhomogeneous standard coordinates on $B$. We call $P$ the spine of $S$; for any $\pi(x) \in S$, we call each subset $\mu^{-1} \mu(\pi(x))=\pi(C x+A) \cap B$ the slice of $S$ through $\pi(x)$ and it is denoted $A_{\pi(x)}$. An $S$-function is any function $c_{0}+c_{1} y_{1}+\cdots+c_{n} y_{n}$ which is constant on all slices of $S$, where $y_{1}, \cdots y_{n}$ is a nonhomogeneous coordinate system on $B$.

The spine $P$ of an equidistant surface $S$ satisfies the two properties

(P1) $P$ is a geodesic line contained in $S$.

(P2) Let $L$ denote the unique $C$-line of $B$ containing $P$, and let $\mu_{L}: B \rightarrow L$ denote the orthogonal projection of $B$ onto $L$. Then $S=$ $\mathrm{U}_{p \in P} \mu_{L}^{-1}(p)$.

The following simple lemma will be useful in showing that the spine of an equidistant surface is uniquely characterized by properties (P1) and (P2).

Lemma 3.2.1. Let $C$ be a $C$-linear subspace of $V$ which contains an open (in the Euclidean topology) subset 0 such that $\pi\left(V^{-} \cap 0\right)$ is a nonempty subset of the equidistant surface $S$. Let $P$ be a subset of $S$ satisfying properties (P1) and (P2), and let $L$ be the $C$-line of $B$ containing $P$. Then $\mu_{L} \pi\left(V^{-} \cap C\right)$ consists of a single point.

Proof. Let $M$ denote the preimage of $L$, i.e., the $C$-linear span of $\pi^{-1}(L)$. Then $\operatorname{dim}_{C} M=2$. It is easy to verify that orthogonal projection $\mu_{L}$ of $B$ onto $L$ can be described as the map $\pi(v) \rightarrow \pi(\lambda(v)$ ), $v \in V^{-}$where $\lambda: V \rightarrow M$ is the orthogonal projection of $V$ onto $M$ with respect to the hermitian form $H$. In particular, $\mu_{L}$ is a holomorphic map. On the other hand, the geodesic line $P$ is an $R$-line and by hypothesis $\mu_{L} \pi\left(V^{-} \cap 0\right) \subset \mu_{L}(S) \subset P$. Consequently $\mu_{L} \pi$ is constant on a nonempty open subset of $C$ and therefore is constant on $V^{-} \cap C$.

Let $P$ be a geodesic line in an equidistant surface $S$ satisfying property (P2) and let $\mu_{L}$ be as in (P2). For any $p \in P$, we call $\mu_{L^{-1}}^{-1}(p)$ the slice at $p$ with respect to $P$.

Lemma 3.2.2. Let $P$ and $P^{\prime}$ be lines in the equidistant surface 
$S$ satisfying (P1) and (P2). Then $P=P^{\prime}$.

Proof. Let $p$ and $q$ be distinct points of $P$ and let $A_{p}, A_{q}$ be the slices at $p, q$ respectively with respect to $P$. Let $C_{p}$ denote the $C$-linear span of $\pi^{-1}\left(A_{p}\right)$. Let $\mu_{L^{\prime}}$ denote the orthogonal projection of $B$ onto the unique $C$-line $L^{\prime}$ containing $P^{\prime}$. By Lemma 3.2.1, one sees that $\mu_{L^{\prime}}\left(A_{p}\right)=\mu_{L^{\prime}} \pi\left(C_{p} \cap V^{-}\right)=p^{\prime}$, a single point of $P^{\prime}$. Consequently $p^{\prime}=A_{p} \cap P^{\prime}$ and $A_{p}=A_{p^{\prime}}$, the slice at $p^{\prime}$ with respect to $P^{\prime}$. Similarly, $q^{\prime}=A_{q} \cap P^{\prime}$ and $A_{q}=A_{a^{\prime}}$, the slice at $q$ with respect to $P$ and the slice at $q^{\prime}$ with respect to $P^{\prime}$.

Consider now the quadrilateral $p, q, q^{\prime}, p^{\prime}$. Since each slice meets $P$ and $P^{\prime}$ at right angles, the sum of the four angles of the quadrilateral is $360^{\circ}$. Hence it lies in a flat subspace of $B$ (cf. [6]). But $B$ has rank 1; that is, the maximal flat geodesic subspaces have $R$ dimension 1 . Hence $P=P^{\prime}$.

Terminology. An equidistant surface is hereafter called a spinal surface.

REMARK. It is clear that the coordinate system in (iii) can be selected to be standardizing.

Let Aut $S$ denote the subgroup of $B$ stabilizing the spinal surface $S$. Then Aut $S$ stabilizes the spine $P$ and indeed operates transitively on $P$. Thus $S$ is the orbit of any of its slices under Aut $S$, and Aut $S$ is the direct product $\operatorname{Aut}_{P} A \times$ Aut $P$ where $A$ denotes the slice through a point $p \in P$ and $A t_{P} A$ the subgroup of Aut $B$ stabilizing $A$ and fixing each point of the normal to $A$ at $p$ (i.e., the $C$-line $L$ containing $P$ ), and Aut $P$ is the one parameter subgroup of Aut $B$ which operates transitively on $P$ and moves each tangent vector to $B$ at a point of $P$ parallel to itself. Explicitly, if $P$ is the $R$-line $\operatorname{Im} x_{1} / x_{0}=0, x_{2}=\cdots=x_{n}=0$ with respect to a standardizing coordinate system $x_{0}, \cdots, x_{n}$ then Aut $P$ is given by the maps

$$
\left\{\begin{array}{l}
x_{0}^{\prime}=a x_{0}+b x_{1}, a, b \in R, a^{2}-b^{2}=1 \\
x_{1}^{\prime}=b x_{0}+a x_{1} \\
x_{i}^{\prime}=x_{i} \quad(i=2, \cdots, n) .
\end{array}\right.
$$

In purely algebraic terms, Aut $S$ is the centralizer in $\mathrm{PU}(H)$ of $T_{R}$ where $T$ is a maximal $R$-split torus in $P U(H)$, i.e., $T_{R}$ is a oneparameter diagonalizable subgroup with positive real eigenvalues. In the terminology of transformation groups, a slice of $S$ is a slice for Aut $S$ operating on $S$. 
Lemma 3.2.3. Let $S$ be a spinal surface, $P$ the spine of $S, A$ a slice of $S, p=P \cap A, L$ the unique $C$-line containing $P$, and let $M, C$ denote the preimage subspaces in $V$ of $L, A$ respectively. Then

(i) $M=(M \cap C)+C^{\perp}$ and $C=(M \cap C)+M^{\perp}$.

(ii) Let $x_{0}$ and $x_{1}$ be independent linear functions on $V$ vanishing on $M^{\perp}$ and let $y_{1}=x_{1} / x_{0}$. Then $y_{1}$ is an S-function.

(iii) If moreover $x_{0}$ vanishes on $(M \cap C)^{\perp}$ and $x_{1}(M \cap C)=0$ then the equation of $S$ is arg $y_{1}=\theta$, where $\theta$ is a constant and the equations of $P$ are:

$$
\arg y_{1}=\theta, y_{2}=y_{3}=\cdots=y_{n}=0
$$

where $y_{i}=x_{i} / x_{0}$ and $x_{2}, \cdots, x_{n}$ is a base for the annihilator of $M(i=2, \cdots, n)$.

Proof. (i) and (ii) are essentially a reformulation of previous remarks (cf. proof of Lemma 3.2.1). As for (iii), let $e_{0}$ be a nonzero element in $M \cap C$ and $e_{1}$ a nonzero element in $C^{\perp}$ such that $\pi\left(e_{0}+e_{1}\right) \in P$. Then $P=\pi\left(\left(e_{0}+\operatorname{Re}_{1}\right) \cap V^{-}\right)$and $y_{1}\left(\pi\left(e_{0}+t e_{1}\right)\right)=x_{1}\left(e_{0}+\right.$ $\left.t e_{1}\right) / x_{0}\left(e_{0}+t e_{1}\right)=t x_{1}\left(e_{1}\right) / x_{0}\left(e_{0}\right)=t x_{1}\left(e_{0}+e_{1}\right) / x_{0}\left(e_{0}+e_{1}\right)=t y_{1}\left(\pi\left(e_{0}+e_{1}\right)\right)$. Thus on $P, \arg y_{1}=\theta$, where $\theta=\arg y_{1}\left(\pi\left(e_{0}+e_{1}\right)\right)$. Since $y_{1}$ is an $S$-function, $\arg y_{1}=\theta$ is the equation of $S$. The conclusion in (iii) is now evident.

LEMMA 3.2.4. Let $S_{1}$ and $S_{2}$ each be spinal surfaces whose spines lie in the same $C$-line and intersect. Then $S_{1}$ and $S_{2}$ meet at a constant Riemannian angle at all points of $S_{1} \cap S_{2}$.

Proof. Let $P_{i}$ denote the spine of $S_{i}$ and let $L$ denote the $C$ line containing $P_{i}(i=1,2)$. Since the geodesic joining two distinct points of $B$ is unique, either $P_{1}=P_{2}$ or $P_{1} \cap P_{2}$ consists of a single point. We need only consider the latter case. Set $\{\mathscr{O}\}=P_{1} \cap P_{2}$. Then $S_{1} \cap S_{2}=\mu_{L}^{-1}(\mathcal{O})$, a common slice. Set $A=S_{1} \cap S_{2}$. Since Aut $B$ acts transitively on (point, line) pairs, we can assume with no loss of generality that the spine of $S_{1}$ is given by the line $0=$ $x_{1}=x_{2}=\cdots=x_{n-1} \operatorname{Im} x_{n} / x_{0}=0$ with respect to a standardizing coordinate system $x_{0}, \cdots, x_{n}$ and that the common slice $A$ is at $x_{n} / x_{0}=0$. Then by Lemma 3.2.3, both $S_{1}$ and $S_{2}$ are $R$-linear in $x_{1} / x_{0}, \cdots, x_{n} / x_{0}$ coordinates.

Let $C$ denote the preimage subspace of $A$ in $V$ and let $H_{C}$ denote the restriction of the hermitian form $H$ to $C$. For any $\pi(x) \in A$ there is a $g_{C} \in U\left(H_{C}\right)$ such that $g_{C} O=\pi(x)$. Then $g_{C}$ is given by equations 


$$
\begin{aligned}
& x_{0}^{\prime}=a_{0 \prime \prime} x_{0}+a_{01} x_{1}+\cdots+a_{-(n-1)} x_{n-1} \\
& \vdots \\
& x_{n-1}^{\prime}=a_{(n-1) 0} x_{0}+\cdots+a_{(n-1)(n-1)} x_{n-1} .
\end{aligned}
$$

We complete these equations to get an element $g$ of $U(H)$ by setting

$$
x_{n}^{\prime}=a x_{n}, \text { where } a=a_{00}\left|a_{00}\right|^{-1} .
$$

With respect to the nonhomogeneous coordinates $x_{1} / x_{0}, \cdots x_{n} / x_{0}$ on $B, g$ acts affinely on $L$. Indeed the element $g$ sends any $p$ with $p \in L$ into the point $g\left(O^{\circ}\right)+a p /\left(a_{00}+0\right)=g(O)+\left|a_{00}\right|^{-1} P$. Thus $g$ sends vectors $O P$ with $p \in L$ into vectors $g(\mathcal{O}) g(p)$ parallel with respect to the coordinate system $x_{1} / x_{0}, \cdots, x_{n} / x_{0}$. Hence $g(p) \in S_{i}$ for $p \in S_{i} \cap L$ since $S_{i}$ is $R$-linear $(i=1,2)$. Consequently, $g$ sends $\left(S_{1} \cap L, S_{2} \cap L\right)$ to $\left(S_{1} \cap g(L), S_{1} \cap g(L)\right)$. Inasmuch as $L$ is the normal to $A$ at $O$ with respect to the Riemannian metric, $g(L)$ is the normal to $A$ at $g\left(O^{\circ}\right)$, and hence by definition $\Varangle\left(S_{1} \cap g(L), S_{a} \cap g(L)\right)$ is the Riemannian angle formed by $S_{1}, S_{2}$ at $g(\mathcal{O})$. Since $g$ induces an isometry on $B$, it preserves Riemannian angles. The proof of Lemma 3.2 .4 is now complete.

Lemma 3.2.5. Let $S$ be a spinal surface and let $I$ be a $C$-line. If $I$ is orthogonal to a slice of $S$, then $I \cap S$ is a geodesic line.

Proof. Let $A$ be a slice of $S$ such that $I$ is orthogonal to $A$ at a point $q$ of $A$. Let $P$ denote the spine of $A$ and set $\mathcal{O}=A \cap P$. Let $y_{1}, y_{2}, \cdots, y_{n}$ be a standard nonhomogeneous coordinate system centered at $\mathcal{O}$ with $y_{1}=y_{2}=\cdots=y_{n-1}=0$ on $P$ and $y_{n}=0$ on $A$. The proof of Lemma 3.2.4 shows that the $C$-line $I$ orthogonal to $A$ at $q$ is given by the equations

$$
y_{1}=y_{1}(q), \cdots, y_{n-1}=y_{n-1}(q),
$$

and moreover, the geodesic lines in $I$ through the point $q$ are given by the additional equation

$$
\arg y_{n}=\text { constant. }
$$

In particular, $I \cap S$ is a geodesic line by Remark 3 of 3.1 .

The common Riemannian angle between $S_{1}$ and $S_{2}$ along $S_{1} \cap S_{2}$ is denoted as $\Varangle\left(S_{1}, S_{2}\right)$; it is called the angle formed by $S_{1}, S_{2}$.

Lemma 3.2.6. Let $S_{i}(i=1,2)$ be a spinal surface in $B$, and assume that $z$ is both an $S_{1}$-function and an $S_{2}$-function. Then the Riemannian angle between $S_{1}$ and $S_{2}$ is given by the euclidean angle 
in the complex $z$-plane of the image sets $z\left(S_{1}\right)$ and $z\left(S_{2}\right)$.

Proof. By definition, $z$ is nonzero polynomial of degree 1 in nonhomogeneous coordinates on $B$ which is constant on all slices. Let $L$ be a $C$-line containing the spine of $S_{1}$. Then $L$ is orthogonal to $S_{1} \cap S_{2}$ at the point $L \cap A$. By definition, as Riemannian angles, $\Varangle\left(S_{1}, S_{2}\right)=\Varangle\left(S_{1} \cap L, S_{2} \cap L\right)$. As a Riemannian space $L$ has constant curvature and the Riemannian angle coincides with the euclidean. The function $z$ restricted to $L$ is a complex analytic map of a $\boldsymbol{C}$-line and is therefore conformal. Consequently

$$
\Varangle\left(S_{1} \cap L, S_{2} \cap L\right)=\Varangle\left(z\left(S_{1} \cap L\right), z\left(S_{2} \cap L\right)\right) .
$$

Since $z$ is an $S_{i}$-function, $z\left(S_{i} \cap L\right)=z\left(S_{i}\right)(i=1,2)$. Consequently, $\Varangle\left(S_{1}, S_{2}\right)=\Varangle\left(Z\left(S_{1}\right), z\left(S_{2}\right)\right)$.

Lemma 3.2.7. Let $S$ be a spinal surface, and let $I$ be a $C$-line, and let $\mu$ denote the projection of $S$ onto its spine.

(i) With respect to any nonhomogeneous coordinate system, $I \cap S$ is an (Riemannian-unbounded circular arc in the disc $I$ or is empty.

(ii) If $I$ meets the spine of $S$, then $I \cap S$ is a geodesic subspace.

(iii) $I \cap S$ is a geodesic line if and only if for any $p \in I \cap S$, the line $p \mu(p)$ and $I \cap S$ span an $R$ - $k$-plane $k \leqq 2$.

(iv) If $I \cap S$ is a geodesic line, then $I \cap S$ and the spine of $S$ span an $\boldsymbol{R}$-k-plane with $k \leqq \inf (3, n)$.

( v) Let $v_{0}, v_{1}, v_{2}$ be elements in $V$ such that $\pi\left(v_{1}\right)$ and $\pi\left(v_{2}\right)$ are distinct points of $I \cap S$ and $\pi\left(v_{0}\right)=\mu\left(\pi\left(v_{1}\right)\right)$. Then $I \cap S$ is a geodesic line if and only if $\left\langle v_{0}, v_{1}\right\rangle\left\langle v_{1}, v_{2}\right\rangle\left\langle v_{2}, v_{0}\right\rangle \in \boldsymbol{R}$.

Proof. Let $P$ denote the spine of $S$. Let $y_{1}, \cdots, y_{n}$ be any standard nonhomogeneous coordinate system centered at a point $\mathscr{O} \in P$ with $y_{n}=0$ on the slice through $\mathcal{O}$. By Lemma 3.2.3, the surface $S$ is $R$-linear in $\operatorname{Re} y_{i}, \operatorname{Im} y_{i}(i=1, \cdots, n)$, and for any $C$ line $I$ the same is also true. Hence $I \cap S$ is $R$-linear. With respect to any other standard nonhomogeneous coordinate system, $I$ is a disc and $I \cap S$ is the transform of a straight line by a fractional linear transformation. Hence $I \cap S$ is an unbounded circular arc.

Assertion (ii) follows from the fact that any straight line in the $y_{1}, \cdots, y_{n}$ coordinate which passes through $\mathcal{O}$ is a geodesic line.

To prove (iii), suppose first that $I \cap S$ is a geodesic line. Let $p, p^{\prime}$ be distinct points on $I \cap S$, and let $\mathcal{O}, \mathcal{O}^{\prime}$ be the orthogonal projections of $p, p^{\prime}$ on $P$. We can choose $e_{0}, v, v^{\prime}$ in $V$ which are 
mapped by $\pi$ onto $\mathcal{O}^{\circ}, p, p^{\prime}$ respectively and standardizing coordinates $x_{0}, \cdots, x_{n}$ with dual base $e_{0}, e_{1}, \cdots, e_{n}$ so that $y_{n}=0$ on the slice through $\mathcal{O}, y_{1}(v) \in \boldsymbol{R}$,

$$
v=e_{n}+a_{1} e_{1}, v^{\prime}=e_{0}+b_{1} e_{1}+b_{n} e_{n}+f
$$

where $a_{1} \in \boldsymbol{R}, b_{n} \in \boldsymbol{R}$, and $f=b_{2} e_{2}+\cdots+b_{n-1} e_{n-1}$. We have $I \cap S \subset$ $\pi\left(v+\boldsymbol{R} v^{\prime}\right)$. Since $I \cap S$ is a geodesic line, $\left\langle v, v^{\prime}\right\rangle \in \boldsymbol{R}$. Clearly $\left\langle e_{1,}, v\right\rangle=\left\langle e_{0}, v^{\prime}\right\rangle=\left\langle e_{0}, e_{0}\right\rangle \in \boldsymbol{R}$. Consequently $\pi\left(\left(\boldsymbol{R} e_{0}+\boldsymbol{R} v+\boldsymbol{R} v^{\prime}\right) \cap V^{-}\right)$ is an $\boldsymbol{R}$-k-plane spanned by $I \cap S$ and $p \mathscr{O}$ of dimension 2 or 1 according as $p \neq 0$ or $p=0$. Set $e^{\prime}=e_{0}+b_{n} e_{n}$. Then $\pi\left(e^{\prime}\right)=C^{\prime \prime}$ and $\left\langle e^{\prime}, v\right\rangle,\left\langle e^{\prime}, v^{\prime}\right),\left\langle e^{\prime}, e_{0}\right\rangle$ are all in $\boldsymbol{R}$. Hence $\pi\left(\left(\boldsymbol{R} e_{0}+\boldsymbol{R} v+\boldsymbol{R} v^{\prime}+\right.\right.$ $\left.\left.R e^{\prime}\right) \cap V^{-}\right)$is an $R$-k-plane with $k \leqq 3$ and $k \leqq 2$ if $n=2$.

Conversely, if $I \cap S$ and $p \mu(p)$ span an $R$-2-plane, then $I \cap S$ is the intersection of two geodesic subspaces and is therefore geodesic.

Assertion ( $v$ ) is clearly a restatement of (iii)

Lemma 3.2.8. Let $A$ and $B$ be orthogonal $C$-planes in $C^{n}$ with $\operatorname{dim}_{c} A=k$ and $\operatorname{dim}_{c} B=n-k$. Let $G$ be a geodesic $R$ - $j$-plane. If $A \cap B \subset G$ and $\operatorname{dim}_{R} A \cap G=k$, then $\operatorname{dim}_{R} B \cap G=j-k$.

Proof. Let $p$ denote the unique point in $A \cap B$, let $T$ denote the tangent space to $C h^{n}$ at $p$, and let $\dot{A}, \dot{B}, \dot{G}$ denote the tangent space at $p$ to $A, B, G$ respectively. It suffices to prove that $\operatorname{dim}_{n} \dot{B} \cap \dot{G}=k-j$. Clearly we can choose $e_{1}, \cdots, e_{j}, \cdots, e_{n}$ an orthonormal base of $\dot{T}$ with $e_{1}, \cdots, e_{j}$ a base for $\dot{G}$, and $e_{1}, \cdots, e_{k}$ a base for $\dot{A}$. Then $e_{k+1}, \cdots, e_{n}$ is a base for $\dot{B}$. Hence $\dot{B} \cap \dot{G}$ has a base $e_{k+1}, \cdots, e_{j}$. Thus $\operatorname{dim}_{k} \dot{B} \cap \dot{G}=j-k$.

LEMMA 3.2.9. Let $S_{\imath}$ be a spinal surface with spine $P_{i}$ and let $L_{2}$ be the $C$-line containing $P_{i}(i=1,2)$. Assume that $L_{1} \cap L_{2}$ contains a point $p_{0}$ which is not on $P_{1}$ or on $P_{2}$. (i) Let $\mathcal{O} \in S_{1} \cap S_{2}$. Then the tangent space to $S_{1}$ and $S_{2}$ at $O$ are distinct. (ii) If $S_{1}$ and $S_{2}$ have a common slice, then $L_{1}=L_{2}$ and $P_{1} \cap P_{2}$ is not empty.

Proof. Suppose not. Let $A_{i}$ denote the slice of $S_{i}$ through $\odot$ $(i=1,2)$. Let $\dot{A}_{\imath}$ and $\dot{S}_{i}$ denote the tangent space to $A_{\imath}$ and $S_{\imath}$ at 0 respectively $(i=1,2)$. Then $\dot{A}_{i}$ is the maximum $C$-linear subspace of $\dot{S}_{i}(i=1,2)$. To prove (i), suppose that $\dot{S}_{1}=\dot{S}_{2}$. Hence $\dot{A}_{1}=\dot{A}_{2}$. This implies that $A_{1}=A_{2}$ since $A_{i}$ is a $C$-linear subspace $(i=1,2)$. Let $\mu_{i}$ denote the orthogonal projection of the ball onto $L_{i} \quad(i=1,2)$. Then $\mu_{i}(\mathscr{O})=A_{i} \cap L_{i} \quad(i=1,2)$. Consequently the geodesic line $p_{0} \mu_{1}(\mathscr{O})$ forms a right angle with the geodesic line $\mu_{2}(\mathcal{O}) \mu_{1}(\mathcal{O})$. Similarly, $\mu_{1}(\mathcal{O}) \mu_{2}(\mathbb{O})$ forms a right angle with $p_{0} \mu_{2}(\mathscr{O})$ 
at $\mu_{2}(\mathscr{O})$. Inasmuch as the sum of the three angles in the geodesic triangle $p_{0} \mu_{1}(\mathcal{O}) \mu_{2}(\mathcal{O})$ is less than or equal to two right angles with equality only if the triangle is degenerate, either $p_{0}=\mu_{i}(\mathscr{O})(i=1$ or 2) or $\mu_{1}(\mathscr{O})=\mu_{2}(\mathscr{O})$. The first possibility is excluded since $\mu_{i}(\mathscr{O}) \epsilon$ $P_{i}(i=1,2)$. Consequently $\mu_{1}(\mathscr{O})=\mu_{2}(\mathcal{O}) \in P_{1} \cap P_{2}$. In particular, $L_{1} \cap L_{2}$ contains the two distinct points $p_{0}$ and $\mu_{1}(\mathcal{O})$. Therefore $L_{1}=L_{2}$ since a $C$-line is uniquely determined by two points. Applying Lemma 3.2.4, one infers that $\Varangle\left(S_{1}, S_{2}\right)=0$ at $\mu_{1}(\mathcal{O})$ as well as at $O$. Hence $P_{1}=P_{2}$ and $S_{1}=S_{2}$.

To prove (ii), let $A$ denote a common slice of $S_{1}$ and $S_{2}$. Choose $\mathscr{O} \in A$, and consider the geodesic triangle $p_{0} \mu_{1}(\mathcal{O}) \mu_{2}(\mathcal{O})$. By the argument above, $\mu_{1}(\mathcal{O})=\mu_{2}(\mathscr{O}) \in P_{1} \cap P_{2}$ and $L_{1}=L_{2}$.

LEMMA 3.2.10. If the spines of two spinal surfaces $S_{1}$ and $S_{2}$ have two distinct common perpendicular lines which meet one spine in two distinct points, then $S_{1}=S_{2}$. In particular, if two spinal surfaces have two common slices, they coincide.

Proof. Let $P_{i}$ denote the spine of $S_{i}(i=1,2)$ and let $g^{1}$ and $g^{2}$ be distinct geodesic lines orthogonal to both $P_{1}$ and $P_{2}$. Then the quadrilateral formed by $g^{1}, P_{1}, g^{2}, P_{2}$ has four right angles. Consequently, it must be degenerate and $P_{1}=P_{2}$. Hence $S_{1}=S_{2}$.

\subsection{Intersections of half-spaces.}

LEMMA 3.3.1. Let $S_{i}$ be a spinal surface with spine $P_{i}(i=1,2)$. Let $L_{i}$ denote the $C$-line containing $P_{i}(i=1,2)$ and assume that $L_{1} \cap L_{2}$ contains a point $p_{0}$. Let $S_{1}^{+}$denote the half-space bounded by $S_{1}$ and containing $p_{0}$, then

(i) for any slice $A_{2}$ of $S_{2}, S_{1}^{+} \cap A_{2}$ is convex (with respect to geodesics in $\mathrm{Ch}^{n}$ );

(ii) $S_{1} \cap S_{2}$ is an unbounded connected $(2 n-2)$ manifold or is empty.

Proof. To prove (i) it suffices to prove $A_{2}^{\prime} \cap S_{1}^{+}$is convex for any $C$-line $A_{2}^{\prime}$ in $A_{2}$. Choose a standard nonhomogeneous coordinate system $y_{1}, y_{2}, \cdots, y_{n}$ centered at $p_{0}$ so that $L_{1}$ has the equation $y_{2}=\cdots=$ $y_{n}=0$. Then the ball $B$ is given by $\left|y_{1}\right|^{2}+\cdots+\left|y_{n}\right|^{2}<1$, the spinal surface $S_{1}$ is given by the equation $\left|y_{1}-a\right|=b$ where $|a|>b$, and $S_{1}^{+}$is given by $\left|y_{1}-a\right|>b$. Moreover the slice $A_{2}$ is a disc orthogonal to the $C$-line $L_{2}$. Consider now the orthogonal projection $\mu_{1}$ of the ball $B$ onto $L_{1}$. The restriction of $\mu_{1}$ to $A_{2}^{\prime}$ is holomorphic and hence a conformal map if $L_{1} \neq L_{2}$; if $L_{1}=L_{2}, \mu_{1}$ maps $A_{2}$ onto a point. In the former case, $\mu_{1}\left(A_{2}^{\prime}\right)$ is a circular disc lying in the 
disc $L_{1}:\left|y_{1}\right|<1$; and $\mu_{1}\left(A_{2}^{\prime}\right) \cap S_{1}^{+}$is therefore a convex subset of $\mu_{1}\left(A_{2}^{\prime}\right)$ with respect to the induced metrics, (which coincides with the Poincaré metric). The inverse image of $\mu_{1}\left(A_{2}^{\prime} \cap S_{1}^{+}\right)$in $A_{2}$ is therefore also convex, because conformal maps of Poincaré discs are isometries. If $L_{1}=L_{2}$, then $\mu_{1}\left(A_{2}\right) \cap S_{1}^{+}=\mu_{1}\left(A_{2}\right)$ or is empty, and correspondingly, $A_{2} \cap S_{1}^{+}=A_{2}$ or is empty. This proves (i).

To prove (ii) for $n=2$, consider the projection $\mu_{1}$ of the spinal surface $S_{1}$ onto its spine $P_{1}$. By Lemma 3.2.7 (i), for each slice $A_{1}$ of $S_{1}, A_{1} \cap S_{2}$ is either an unbounded circular arc in $A_{1}$ or is empty. From this (ii) follows. For larger $n$, a similar inductive argument can be given. Proof of the lemma is now complete.

LEMMA 3.3.2. Let $\Delta$ be a finite subset of isometries of $\mathrm{Ch}^{n}$. Let $p_{0} \in C h^{n}$. For any $\gamma \in \Delta$, set

$$
\begin{aligned}
& \gamma^{+}=\left\{x \in C h^{n} ; d\left(x, p_{0}\right) \leqq d\left(\gamma x, p_{0}\right)\right\} \\
& \hat{\gamma}=\left\{x \in C h^{n} ; d\left(x, p_{0}\right)=d\left(\gamma x, p_{0}\right)\right\} \\
& F(\Delta)=\bigcap_{\gamma \in J} \gamma^{+} .
\end{aligned}
$$

Then $F(\Delta)$ and each of its $k$-dimensional faces is topologically a cell, $k=0,1,2, \cdots, 2 n$.

Proof. We prove the result for $n=2$. For larger $n$, an inductive argument based on similar considerations can be given.

The region $F$ is clearly star-shaped with respect to geodesics originating at $p_{0}$. Inasmuch as the boundary of $F$ is made up of a finite number of piecewise smooth 3 -surfaces, $F$ is a 4-cell.

Let $e^{3}$ be a 3 -face of $F$; that is, $e_{3}$ is a connected component of $\hat{\gamma}_{0} \cap \bigcap_{\gamma \in \Delta} \gamma^{+}$.

Let $\mu$ denote the projection of the spinal surface $\hat{\gamma}_{0}$ onto its spine $P$. By Lemma 3.3.1, each slice of $\hat{\gamma}_{0}$ meets $\gamma^{+}$in a convex set and consequently each fiber of the map $\mu$ is a cell. Since $e^{3}$ is connected, $\mu\left(e^{3}\right)$ is an interval. It follows that $e^{3}$ is a cell. The proofs for 2-cells and 1 cells are similar.

4. Arithmeticity of groups generated by $C$-reflections.

Let $G$ be a semi-simple real Lie group and let $\Gamma$ be a lattice subgroup of $G$.

Definition. $\quad \Gamma$ is an arithmetic lattice in $G$ if and only if there is an algebraic matrix group $A$ defined over the field $\boldsymbol{Q}$ of rational numbers and containing Ad $G$, the adjoint group of $G$ such that

(i ) $A_{R}=\operatorname{Ad} G \times K$ (direct) with $K$ compact. 
(ii) $\operatorname{Ad} \Gamma$ is commensurable with the projection of $A_{z}$ into $G$ i.e., $\operatorname{Ad} \Gamma \cap\left(A_{z} K \cap G\right)$ is of finite index in both $\Gamma$ and $A_{z} K \cap G$.

REMARK. Let $G^{*}$ denote the Zariski-closure of $\mathrm{Ad} G$ in the full matrix algebra $\mathscr{E}$ over complex numbers and let $k$ denote the field generated by $Q[\operatorname{Tr} A d \Gamma]$. Let $\mathcal{O}$ denote the ring of algebraic integers of $k$ and let Gal $k$ denote the set of all monomorphisms of $k$ into $C$. It is known (cf. (2.5.1)) that the algebraic group $G^{*}$ can be defined over the field $k$. Let $I$ denote the ideal of polynomial functions (in the entries of matrices in $\mathscr{E}$ ) with coefficients in $k$ which vanish on $G^{*}$ and let ${ }^{\sigma} I$ denote the image of $I$ under $\sigma \in$ Gal k. One denotes by ${ }^{\sigma} G^{*}$ the algebraic matrix group on which the ideal ${ }^{\circ} I$ vanishes; ${ }^{\sigma} G^{*}$ is defined over the field ${ }^{\circ} k$.

In this paper, the group $\operatorname{Ad} G$ will usually be $\operatorname{PU}(n, 1)$ and $G^{*}=$ PGL $(n+1, C)$ which is a simple group. Whenever $G^{*}$ is a simple group, the groups $A$ and $K$ in the definition of arithmetic group are easy to identify. Set $\mathscr{G}=$ Gal $k$, and

$$
\begin{aligned}
& \mathscr{G}_{R}=\{\sigma \in \text { Gal } k ; \bar{\sigma}=\sigma\} \\
& \mathscr{G}_{-}=\left\{\sigma \in \mathscr{G}_{R} ;\left({ }^{\circ} G^{*}\right)_{R} \text { is compact }\right\} .
\end{aligned}
$$

For $\sigma \in \mathscr{G}-\mathscr{G}_{\boldsymbol{R}},{ }^{\sigma} G^{*} \times{ }^{\sigma} G^{*}$ is defined over $\boldsymbol{R}$, and, as is easy to see, $\left({ }^{\circ} G^{*} \times{ }^{\sigma} G\right)_{R}=\left({ }^{\sigma} G^{*}\right)_{c}$. It is well-known (and easy to prove) that a connected compact complex group is abelian. Thus $\sigma \in \mathscr{G}-\mathscr{G}_{R}$ implies that $\left({ }^{\sigma} G^{*} \times{ }^{\sigma} G^{*}\right)_{R}$ is not compact. Consequently if we form

$$
A=\prod_{\sigma \in \mathscr{G}}{ }^{\sigma} G^{*}
$$

and set

$$
K=\prod_{\sigma \in \mathscr{G}_{-}}\left({ }^{\sigma} G^{*}\right)_{R}, B=\prod_{\sigma \in \mathscr{G}-\mathscr{G}_{-}}{ }^{\sigma} G^{*}
$$

then $B$ is defined over $R$ and the algebraic group $A$ is defined over $\boldsymbol{Q}$; it is the group obtained from $G^{*}$ by "restriction from $k$ to $\boldsymbol{Q}$ ". Moreover, $B_{R}$ is the product of all the noncompact factors of $A_{R}$. By definition, the subgroup $A_{z} K \cap B$ is an arithmetic lattice in $B_{R}$, the fact that it is a lattice following from the theorem of BorelHarish Chandra that $A_{R} / A_{z}$ has finite measure (cf. [2]).

If $G^{*}$ is simple and $k=Q[\operatorname{Tr} \operatorname{Ad} \Gamma]$, then $B_{R}=\operatorname{Ad} G, \mathscr{G}_{-}=\mathscr{G}_{-}$ \{identity\} and thus ${ }^{\sigma} k \subset \boldsymbol{R}$ for all $\sigma \neq$ identity. Since $G$ is a real Lie group, $k \subset \boldsymbol{R}$, thus $k$ is a totally real field with ${ }^{\sigma}\left(G^{*}\right)_{\boldsymbol{R}}$ compact for all $\sigma \in \mathscr{G}_{-}$.

The following lemma provides us with a test for arithmeticity of a lattice generated by complex reflections.

LEMMA 4.1. Let $H$ be a nondegenerate hermitian form $\Sigma a_{i j} z_{i} \bar{z}_{j}$ 
on $C^{n}$, let $h=\boldsymbol{Q}\left\{\left\{a_{i j}\right\} ; i, j=1, \cdots, n\right\}$ and let $G=U(H)$, the unitary group of $H$. Let $\Gamma$ be a lattice subgroup of $G$, let $k=Q[\operatorname{Tr} A d \Gamma]$ and let $O$ be the ring of algebraic integers in $k$. Let $E$ denote the composite field of $h$ and $k$. Assume

(1) The field $k$ is totally real.

(2) For all $x \in h$ and $\sigma \in \operatorname{Gal} h, \sigma(\bar{x})=\overline{\sigma(x)}$.

(3) $\operatorname{Tr}[\operatorname{Ad} \Gamma] \subset \mathcal{O}$.

Then $\operatorname{Ad} \Gamma$ is arithmetic in $\operatorname{Ad} G$ if and only if:

For all $\sigma \in$ Gal $E$ with $\sigma \neq 1$ on $k,{ }^{\sigma} H$ is definite.

Proof. Let $G^{*}$ denote the Zariski-closure of Ad $G$. Then $G^{*}=$ PGL $(n, C)$, a simple group. Let $A=\boldsymbol{R}_{k / \mathbf{Q}} G$, the group defined over $\boldsymbol{Q}$ by restricting the ground field from $k$ to $\boldsymbol{Q}$. Then

$$
A=\prod_{\sigma \in \varrho}{ }^{\sigma} G^{*}
$$

where $=$ Gal $k$. For any $\sigma \in$ Gal $E$ which is not the identity on $k,{ }^{\sigma} G^{*}$ is defined over $\boldsymbol{R}$ by hypothesis (1) and ${ }^{\circ} H$ is a hermitian form by (2), $\left(G^{*}\right)_{R}=\mathrm{PU}(H)$ and $\left({ }^{\circ} G^{*}\right)_{R}=\mathrm{PU}\left({ }^{\circ} H\right)$.

Assume ${ }^{\sigma} H$ is definite for $\sigma \neq 1$ on $k$. Then ${ }^{\sigma}\left(G^{*}\right)_{R}$ is compact for all $\sigma \in \mathrm{Gal} k$ with $\sigma \neq 1$. Let $\Gamma^{\prime}$ denote the projection of $A_{z}$ into the $\sigma=1$ factor. Since $\Pi_{\sigma \neq 1}\left({ }^{\sigma} G^{*}\right)_{R}$ is compact, $\Gamma^{\prime}$ is a discrete subgroup. The $\boldsymbol{Q}$-structure on $A$ arises from the $\boldsymbol{Q}$-structure on the field $k$ regarded as a $Q$-vector space, embedded into

$$
\prod_{\sigma \in \mathscr{G}}{ }^{\sigma} k \approx k \underset{Q}{\otimes} C
$$

via the diagonal embedding $x \rightarrow \Pi_{\sigma \in \mathscr{S}}{ }^{\sigma} x$. Hence $\Gamma^{\prime}=\left(G^{*}\right)_{\sigma}$. By hypothesis (3), $\Gamma \subset \Gamma^{\prime}$. To prove that $\Gamma$ is arithmetic, it remains only to prove that $\Gamma^{\prime} / \Gamma$ is a finite set. By hypothesis, $G / \Gamma$ has finite Haar measure. Hence $\operatorname{Ad} G / \operatorname{Ad} \Gamma$ has finite Haar measure. We have $G_{R}^{*}=\operatorname{Ad} G$, and $(\operatorname{Ad} G) / \Gamma^{\prime}$ has finite measure. Moreover

$$
\mu\left(\operatorname{Ad} G / \Gamma^{\prime}\right) \cdot \mu\left(\Gamma^{\prime} / \Gamma\right)=\mu(\operatorname{Ad} G / \Gamma)
$$

where "' denotes Haar measure. It follows that $\Gamma^{\prime} / \Gamma$ is finite. Hence $I$ is arithmetic.

Conversely, assume that $\mathrm{Ad} \Gamma$ is arithmetic in $\operatorname{Ad} G$. Then ${ }^{\circ}$ Ad $\Gamma$ is bounded for all $\sigma \in$ Gal $k$ with $\sigma \neq 1$. Since the map of $\mathrm{GL}(n, C)$ to PGL $(n, C)$ has compact kernel and since ${ }^{\sigma} \mathrm{Ad} \gamma=\operatorname{Ad} \sigma \gamma$, the matrices ${ }^{\sigma} T$ are bounded in PU $\left({ }^{\sigma} H\right)$ for any $\sigma \in$ Gal $E$ with $\sigma \neq$ 1 on $k$. Hence the topological closure ${ }^{\sigma} \Gamma$ is a compact group and as is well-known stabilizes a positive definite hermitian form on $\boldsymbol{C}^{n}$. But ${ }^{\circ}{ }^{\prime}$ is irreducible on $C^{n}$ (cf. (2.3.3)) and therefore stabilizes aunique hermitian form up to a scalar factor. Consequently ${ }^{\sigma} H$ is definite. 
REMARK 1. The proof of Lemma 4.1 shows that the group Ad $\Gamma$ is commensurable to $G_{\mathscr{Q}}^{*}$ if $G^{*}$ is simple and $\mathcal{O}$ is the ring of integers in $Q[\operatorname{Tr} A d \Gamma]$.

By the same argument one can prove:

Let $G$ be a semi-simple matrix group defined over a field $k$, let $O$ denote the ring of algebraic integers in $k$. If

(1) $k$ is totally real

(2) ${ }^{\sigma} \operatorname{Tr} \operatorname{Ad} \Gamma$ is bounded for all $\sigma \in$ Gal $k$ with $\sigma \neq 1$, then $G_{0}$ is discrete (and an arithmetic lattice in $G$ ).

REMARK 2. Let $k$ be a subfield of $R$ and $\mathcal{O}$ the ring of integers of $k$. Let $G$ be an algebraic matrix group defined over $k$. If $G_{0}$ is discrete, it is an arithmetic lattice in $G_{R}$. The proof involves "weak approximation" i.e., a generalization of the Chinese remainder theorem to algebraic groups.

Lemma 4.3. Let $\mathscr{D}$ be a Coxeter diagram, let $H$ be a nondegenerate hermitian form associated to $\mathscr{D}$, and let $\Gamma$ be the group generated by $C$-reflections associated to $(\mathscr{D}, H)$ and rational phaseshifts. Then for all $\gamma \in \Gamma, \operatorname{Tr} \gamma$ is an algebraic integer, and moreover, it is a sum of roots of unity if $p_{1}=p_{2}=\cdots$.

Proof. Let $n$ be the number of nodes of $\mathscr{D}$, let $e_{1}, \cdots, e_{n}$ denote the canonical base of $C^{n}$, and let $\left\{p_{i}, q_{i j}, i, j=1, \cdots, n, i \neq j\right\}$ denote the data of $\mathscr{D}$. Then

$$
\left\langle e_{i}, e_{j}\right\rangle=H\left(e_{i}, e_{j}\right)=\alpha_{i j} \varphi_{i j},\left|\varphi_{i j}\right|=1
$$

and

$$
\begin{aligned}
& \alpha_{i i}=1, \quad \varphi_{i i}=1 \\
& \alpha_{i j}=-\frac{c_{i j}}{s_{i j}}, i \neq j
\end{aligned}
$$

where

$$
\begin{aligned}
c_{i j} & =\left(\frac{\cos \left(\pi / p_{i}-\pi / p_{j}\right)+\cos 2 \pi / q_{i j}}{2}\right)^{1 / 2} \\
s_{i j} & =\left(\sin \pi / p_{i} \sin \pi / p_{j}\right)^{1 / 2} .
\end{aligned}
$$

By definition, the $C$-reflection $R_{i}$ in $e_{i}^{\perp}$ is:

$$
R_{i} x=x+\left(\eta_{i}^{2}-1\right)\left\langle x, e_{i}\right\rangle e_{i}
$$

that is, $R_{i}=1+e_{i} \otimes \beta_{i}$, where $\beta_{i}$ is the linear map $x \rightarrow\left(\eta_{i}^{2}-1\right)\langle x$, $\left.e_{i}\right\rangle$, and $e \otimes \beta$ denote the endomorphism of $C^{n}:(e \otimes \beta)(v)=\beta(v) e$. In 
this notation, $(e \otimes \beta)(f \otimes \alpha)=\beta(f) e \otimes \alpha$. Thus

$$
\operatorname{Tr} R_{i_{1}} R_{i_{2}} \cdots R_{i_{m}}=\sum_{i=1}^{m} \sum_{1 \leq j_{1}<j_{2}<\cdots<j_{l} \leq m} \beta_{i_{j_{1}}}\left(e_{i_{j_{2}}}\right) \beta_{i_{j_{2}}}\left(e_{i_{j_{3}}}\right) \cdots \beta_{i_{j_{l}}}\left(e_{i_{j_{1}}}\right) .
$$

Thus $\operatorname{Tr} \gamma$ is a sum of terms of the type

$$
\pm\left(\eta_{i_{1}}^{2}-1\right) \cdots\left(\eta_{i_{p}}^{2}-1\right) \alpha_{i_{1} i_{2}} \alpha_{i_{2} i_{3}} \cdots \alpha_{i_{p} i_{1}} \rho_{i_{1} i_{2}} \cdots \rho_{i_{p} i_{1}}
$$

or

$$
\pm \prod_{j=1}^{p}\left(\eta_{i_{j}}^{2}-1\right) \frac{1}{\sin \frac{\pi}{p_{i_{j}}}} \cdot \prod_{j=1}^{p} c_{i_{j i} i_{j+1}}
$$

Now

$$
\begin{aligned}
\left(\eta_{j}^{2}-1\right) \cdot \frac{1}{\sin \frac{\pi}{p_{j}}} & =\eta_{j}\left(\eta_{i}-\bar{\eta}_{j}\right) \frac{2 \sqrt{-1}}{\eta_{j}-\eta_{j}} \\
& =2 \eta_{j} \sqrt{-1} .
\end{aligned}
$$

Hence $\operatorname{Tr} \gamma$ is a sum of terms of the form $i^{m} \prod_{j=1}^{p} \eta_{i j} \cdot \prod_{j=1}^{p}\left(2 c_{i_{j} i j+1}\right)$. Since $2 c_{i j}$ is an algebraic integer and moreover, a sum of roots of unity, if $p_{1}=p_{2}=\cdots=p_{n}$ (by the $\cos (\theta / 2)$ formula) the same is true of $\operatorname{Tr} \gamma$ provided $\prod_{j=1}^{p} \varphi_{i_{i} i_{j+1}}$ is a root of unity.

This last condition is assured by the hypothesis of rational phase shifts. Proof of the lemma is now complete.

LemMa 4.4. Let $\Gamma$ be a group generated by C-reflections associated to a Coxeter diagram $\mathscr{D}$ with $n+1$ nodes and Hermitian form $H$. Assume

(1) $H$ is of type ( $n$ plus, 1 minus).

(2) The group $\Gamma^{i}$ generated by the n-reflections $R_{1}, \cdots, \widehat{R_{i}}, \cdots, R_{n+1}$ of the reflections $R_{1}, \cdots, R_{n+1}$ has a connected diagram and is finite.

(3) $H$ has rational phase shifts.

(4) $p_{1}=p_{2}=\cdots=p_{n+1}$.

Then $\operatorname{Ad} \Gamma$ is arithmetic in $\mathrm{PU}(H)$ if and only if: For all automorphisms of $\boldsymbol{C}$ which are not the identity on the field $\mathbf{Q}[\operatorname{Ad} \Gamma]$, ${ }^{\circ} H$ has positive determinant.

Proof. Let $W=C e_{1}+\cdots C e_{\hat{i}}+\cdots C e_{n+1}$. By (2.3.3) and hypothesis (2), $\Gamma^{i}$ is irreducible on $W$. Consequently $\Gamma^{i}$ stabilizes a unique hermitian form on $W$ modulo a scalar factor. Since $\Gamma^{i}$ is a finite group, it stabilizes a positive definite hermitian form on $W$. Hence any hermitian form on $W$ stabilized by $\Gamma^{i}$ is definite. Then the restriction of ${ }^{\sigma} H$ to $W$ is definite for each automorphism $\sigma$ of 
C. Inasmuch as $\left\langle e_{i}, e_{i}\right\rangle=1$ for all $i,{ }^{\circ} H$ is positive definite on $W$ and is of type ( $n$ plus, 1 minus) or else positive definite for each automorphism $\sigma$ of $C$; these two possibilities correspond to ${ }^{\sigma} \Delta<0$ and ${ }^{\sigma} \Delta>0$ respectively, where $\Delta$ denotes the determinant of the $(n+1) \times(n+1)$ matrix $H\left(e_{i}, e_{j}\right)$.

Let $k=\boldsymbol{Q}[\operatorname{Tr} \operatorname{Ad} \Gamma] . \quad$ By Lemma $4.2, k=\boldsymbol{Q}\left[|\operatorname{Tr} \Gamma|^{2}\right] . \quad$ By hypothesis (4) and Lemma $4.3, k$ consists of sums of real parts of roots of unity. Hence $k$ is totally real. By hypothesis (3) and (4) $H\left(e_{i}, e_{j}\right)$ lies in a field generated by roots of unity for $i, j=1, \cdots, n+1$; let $k$ denote the field generated by $h_{i j}$. Then for all $\sigma \in \mathrm{Gal} h$ and $x \in h, \sigma(\bar{x})=\overline{\sigma(x)}$. Thus hypotheses (1), (2), and (3) of Lemma 4.1 are satisfied. Let $E$ denote the field generated by $h$ and $k$. We have seen that for all $\sigma \in \mathrm{Gal} E,{ }^{\circ} H$ is either of type $(n, 1)$ or positive definite according as ${ }^{\sigma} \Delta<0$ or ${ }^{\circ} \Delta>0$. The conclusion of the lemma follows from Lemma 4.1.

\section{Verification of discreteness.}

In the search for nonarithmetic lattices among groups $\Gamma$ generated by $\boldsymbol{C}$-reflections, we can test a lattice for arithmeticity by the criterion of Lemma 4.4. But how do we test whether $\Gamma$ is a lattice? Most particularly, is there a test for $\Gamma$ to be discrete?

It may be of interest to describe an algorithm applicable to a wide class of $\Gamma$ which is finite if and only if $\Gamma$ is discrete.

It is based on the following general simple lemma.

LEMMA. Let $X$ be a connected simply connected metric space and $\Gamma$ a group of isometries of $X$. Let $F$ be a closed subset of $X$ and $\triangle$ a finite subset of $\Gamma$ satisfying

(1) $F$ lies in the interior of $\Delta F=\bigcup_{i \in\lrcorner} \gamma F$.

(2) If $\gamma_{1}, \gamma_{2} \in \Delta$ and $\gamma_{1} F \cap \gamma_{2} F \neq \phi$, then $\gamma_{1}^{-1} \gamma_{2} \in \Delta$.

(3) The induced metric on $F$ modulo $\triangle$ is complete. ${ }^{1}$

Then $\triangle$ generates $\Gamma$ and $\Gamma$ is discrete.

Proof. On the space $\Gamma \times F$ define the equivalence relation $\left(\gamma_{1}\right.$, $\left.x_{1}\right) \equiv\left(\gamma_{2}, x_{2}\right)$ if and only if $\gamma_{1} x_{1}=\gamma_{2} x_{2}$ and $\gamma_{1}^{-1} \gamma_{2} \in \Delta$. Let $Y$ denote the quotient space $\bmod \equiv$ of $\Gamma \times F$ and let $\eta: \Gamma \times F \rightarrow Y$ the quotient map. Let $\pi$ denote the map of $Y$ to $X$ induced by $(\gamma, x) \rightarrow \gamma x$.

$\Gamma$ acts on $Y$ in the obvious way and $\pi$ is a $\Gamma$ map. It is easy to see that $\Gamma F$ is open and by (3) is closed in $X$. Hence $\Gamma F=X$.

Moreover, the map $\pi$ is a covering map. To prove this, it suffices to prove it is an even covering at each interior point of $F$ in view of hypothesis (1) and $\Gamma F=F$.

\footnotetext{
${ }^{1}$ I am indebted to Bernard Maskit for pointing out the necessity of this hypothesis.
} 
On $\eta(\Delta \times F)$, the map $\pi$ is a homeomorphism by definition of the quotient topology. Given $\eta(\gamma, x) \in \pi^{-1}\left(x^{\prime}\right)$ with $\gamma \in \Gamma$ and $x, x^{\prime} \in F$, then $\gamma x=x^{\prime}$ and hence $\eta(\gamma \Delta, F)$ maps homeomorphically onto $\gamma \Delta F$. The latter contains $\gamma F$ in its interior. Indeed $U=\bigcap_{\substack{\gamma \in \Gamma \\ \gamma x=x^{\prime}}} \gamma \Delta F$ is a neighborhood of $x^{\prime}$; since $\Gamma \subset$ Isom $X, U$ contains a ball centered at $x^{\prime}$ of radius $d\left(F, x^{\prime}-\Delta F\right)$. Then $\pi$ maps each connected component of $\pi^{-1}\left(U^{\prime}\right)$ homeomorphically onto $U^{\prime}$.

Since $X$ is simply connected, $\pi$ is a homeomorphism. Clearly $\Gamma$ is discontinuous on $Y$. Hence it is discontinuous on $X$.

Suppose next that $\Gamma$ is a group of isometries on $X=\boldsymbol{C} h^{n}$. Let $F$ be a compact region in $X$ and let $\Delta_{1}$ be a finite subset of $\Gamma$ satisfying

(1) $F$ lies in the interior of $\Delta_{1} F$.

(2) $\Delta_{1}=\Delta_{1}^{-1}$.

Set $\Delta_{1}^{2}=\left\{\gamma \gamma^{\prime} ; \gamma, \gamma^{\prime} \in \Delta_{1}\right\}$.

$$
E_{1}=\left\{\gamma \in \Delta_{1}^{2}-\Delta_{1} ; \gamma F \cap F \neq \dot{\phi}\right\} .
$$

Inductively set

$$
\begin{aligned}
& \Delta_{i+1}=\Delta_{i} \cup E_{i} \\
& E_{i+1}=\left\{\gamma \in \Delta_{i+1}^{2}-\Delta_{i} ; \gamma F \cap F \neq \phi\right\} .
\end{aligned}
$$

Proposition. $\Gamma$ is discrete if and only if $E_{i}$ is empty for some $i,(i=1,2, \cdots)$.

Proof. (њ). This assertion follows immediately from the lemma. $(\rightarrow)$. This assertion follows immediately from the definition of discontinuous group.

This algorithm for proving discreteness is impractical because if the group $\Gamma$ is not discrete, the algorithm never comes to a conclusion. However, a refinement of the lemma on which it is based leads under the added hypotheses of $\S 6$, to a criterion which is more effective i.e., it implies discreteness in some cases, and enables us to prove nondiscreteness in others by suggesting where to look for pairs of $\boldsymbol{C}$-reflections with nonadmissible values.

6. Joined spaces and a discreteness criterion.

\subsection{Abutted families of polyhedra.}

For any $n$-dimensional polyhedron $F$ we denote by $E_{k}(F)$ the set of its $k$-condimensional faces. We consider only finite polyhedra $F$ which are cells minus faces and whose faces are also of this type. In particular, each $e \in E_{2}(F)$ lies on exactly two elements of $E_{1}(F)$. Write $E_{n+1}(F)$ for the empty set $\varnothing$ and set $E_{0}(F)=F$. 
Definition. Let $X$ be a topological space. An abutted family $\mathscr{F}$ of polyhedra in $X$ is a family $\mathscr{F}$ of polyhedra together with a distinguished subset $\mathscr{N}$ of $\mathscr{F} \times \mathscr{F}$ satisfying;

(1) If $\left(F, F^{\prime}\right) \in \mathscr{N}$, then $F \neq F^{\prime}$ and $\left(F^{\prime}, F\right) \in \mathscr{N}$.

(2) If $\left(F, F^{\prime}\right) \in \mathscr{N}$, then $F \cap F^{\prime} \in E_{1}(F) \cap E_{1}\left(F^{\prime}\right)$.

(3) If $\left(F, F^{\prime}\right) \in \mathscr{N}$ and $\left(F, F^{\prime \prime}\right) \in \mathscr{N}$ and $F \cap F^{\prime}=F \cap F^{\prime \prime}$, then $F^{\prime}=F^{\prime \prime}$.

(4) For each $e \in E_{1}(F)$, there is an $F^{\prime} \in \mathscr{F}$ with $F \cap F^{\prime}=e$ and $\left(F, F^{\prime}\right) \in \mathscr{N}$.

The $F^{\prime}$ in (4) is necessarily unique and is denoted $e(F) . \quad \mathfrak{n}$ is called the adjacency of $\mathscr{F}$; two elements of $\mathscr{F}$ are called adjacent if and only if $\left(F, F^{\prime}\right) \in \mathscr{N}$ :

For any $F_{0}$ and $F$ in $\mathscr{F}$ and $e \in E_{k}\left(F_{0}\right) \cap E_{k}(F)$, we say that $F_{0}$ and $F$ are $e$-connected, if there if a sequence $F_{1}, F_{2}, \cdots, F_{n}=F$ with $\left(F_{i}, F_{i+1}\right) \in \mathscr{N}(i=1,2, \cdots, n-1)$ and $e \in E_{k}\left(F_{0}\right) \cap \cdots \cap E_{k}\left(F_{1}\right) \cap$ $E_{k}\left(F_{n}\right)$; in case $k=n+1$, we say simply that $F_{0}$ and $F$ are connected. We called the abutted family $\mathscr{F}$ connected if every two of its elements are connected.

We set for any $F \in \mathscr{F}$

$$
\mathscr{N}(F)=\left\{e(F) ; e \in E_{1}(F)\right\} .
$$

For any subset $\mathscr{S} \subset \mathscr{F}$, we write

$$
\mathscr{N}(\mathscr{S})=\{\mathfrak{L}(F) ; F \in \mathscr{S}\} \text {. }
$$

A subfamily $\mathscr{S} \subset \mathscr{F}$ is called open if $\mathscr{N}(\mathscr{S})=\mathscr{S}$. Clearly any open subfamily of $\mathscr{F}$ which contains $F$ contains any connected subfamily $\mathscr{C}$ with $F \in \mathscr{C}$.

\subsection{The joined $\mathscr{F}$-space.}

Let $\mathscr{F}$ be an abutted family of polyhedra in a topological $n$ manifold $X$. Let $X \times \mathscr{F}$ denote the topological direct product of the spaces $X$ and $\mathscr{F}$ where $\mathscr{F}$ is given the discrete topology. Set

$$
D=\bigcup_{F \in \sim} F x\{F\}=\{(x, F) ; x \in F, F \in \mathscr{F}\} .
$$

On the topological space $D$ consider the relation $\equiv$ which is generated by the equivalences

$$
(x, F)=\left(x^{\prime}, F^{\prime}\right) \text { if } x=x^{\prime} \text { and } x \in E_{1}(F) \cap E_{1}\left(F^{\prime}\right) .
$$

Set $Y=D \bmod \equiv$. Let $\eta$ denote the canonical map of $D$ to $Y$.

Definition. The space $Y$ is called the joined $\mathscr{F}$-space.

The projection $X \times \mathscr{F} \rightarrow X$ induces a well-defined continuous 
map $\pi$ of $Y$ into $X$. We call $\pi$ the canonical map of the joined $\mathscr{F}$ space into $X$.

For any face $e$ of an $F \in \mathscr{F}$, let $F_{e}$ denote the union of the interiors (of $F$ and) of all faces of $F$ which contain $e$. Set

$$
\mathscr{F}_{e}=\left\{F_{e} ; F \in \mathscr{F}, e \subset F\right\} \text {. }
$$

Then $\mathscr{F}_{e}$ is an abutted family of polyhedra on the space $X_{e}$ which is the union of $\left\{\pi\left(F_{e}\right) ; F_{e} \in \mathscr{F}_{e}\right\}$, when we restrict adjacency in $\mathscr{F}_{e}$ from $\mathscr{F}$.

For any $e \in E_{k}(F)$, let $\mathscr{F}_{e, F}$ denote the maximum connected subfamily of $\mathscr{F}_{e}$ which contains $F_{e}$. Clearly $\mathscr{F}_{e, F^{\prime}}=\mathscr{F}_{e, F}$ for all $F^{\prime} \in$ $\mathscr{F}_{e}$. Let $Y(e, F)$ denote the joined $\mathscr{F}_{e, F}$ space. It is easy to see that $Y(e, F)$ is a neighborhood in $Y$ of $\{\eta(x, F) ; x \in$ interior of $e\}$.

Suppose that $X$ is a topological manifold. Let $F \in \mathscr{F}, e \in E_{k}(F)$, and let $e^{\perp}$ be a closed small (topological) $k$ ball transversal to $e$. Set $S_{e}=\partial e^{\perp} . \quad S_{e}$ is a $(k-1)$-sphere. Set

$$
\begin{aligned}
& \mathscr{F}_{e}\left(S_{e}\right)=\left\{F \cap S_{e} ; F \in \mathscr{F}_{e}\right\} ; \\
& \mathscr{F}_{e, F}\left(S_{e}\right)=\left\{F \cap S_{e} ; F \in \mathscr{F}_{e, F}\right\} .
\end{aligned}
$$

Definition. The abutted family $\mathscr{F}$ on $X$ is called smooth if it satisfies the following conditions:

(1) The polyhedra of $\mathscr{F}$ are "nice" in the sense that for any $F \in \mathscr{F}, e \in E_{k}(F)$, and "nicely" embedded small transversal $k$ ball $e^{\perp}, F \cap S_{e}$ is a polyhedron.

(2) $\mathscr{F}_{e}\left(S_{e}\right)$ is an abutted family of polyhedra on the $(k-1)$ sphere $S_{e}$, and combinatorially, this family is independent of the choice of the nicely embedded small transversal $k$ ball $e^{\perp}$.

(3) Let $S(e, F)$ denote the joined $\mathscr{F}_{e, F}\left(S_{e}\right)$ space. Then $Y(e, F)$ is homeomorphic to the direct product Int $e \times S(e, F)$, where Int denotes interior.

\subsection{A criterion for discreteness.}

THEOREM 6.3.1. Let $\mathscr{F}$ be a connected abutted smooth family of compact polyhedra in the (connected) simply connected topological n-manifold $X$. Assume that $X$ has a metric and that there exists a positive number $r$ such that each $F \in \mathscr{F}$ contains a ball of radius $r$. Assume also that $\mathscr{F}$ satisfies the condition

(CD2) For any $F_{0} \in \mathscr{F}$ and $e \in E_{2}\left(F_{0}\right)$, and any sequence $F_{0}, F_{1}$, $F_{2}, \cdots, F_{n}$ of successively adjacent polyhedra with $e \in E_{2}\left(F_{0}\right) \cap E_{2}\left(F_{1}\right) \cap$ $\cdots \cap E_{2}\left(F_{n}\right)$, if $F_{0} \cap F_{n}$ has a nonempty interior, then $F_{0}=F_{n}$.

Let $Y$ denote the jointed $\mathscr{F}$-space. Then the canonical map $\pi: Y \rightarrow X$ is a homeomorphism of $Y$ onto $X$. 
Proof. We use induction on $n=\operatorname{dim} X$. If $\operatorname{dim} X=1$, then $X$ is homeomorphic to the real line, and the hypotheses imply that $\mathscr{F}$ is a locally finite covering of the line by intervals which meet only at their end points.

Now assume the result for spaces of dimension less than $n$. If $k=2$, then by hypothesis (CD2) any maximal connected subfamily of $\mathscr{F}_{e}$ gives a cell decomposition with disjoint interiors of the circle $S_{e}$. For $k \geqq 2$ and $e \in E_{k}$, it is clear that the family $\mathscr{F}_{e}\left(S_{e}\right)$ inherits property (CD2) from $\mathscr{F}$. Thus any maximal connected subfamily of $\mathscr{F}_{e}\left(S_{e}\right)$ yields a finite cell decomposition with disjoint interiors of the $(k-1)$-sphere $S_{e}$.

It follows, using the smoothness, that for any $F \in \mathscr{F}, e \in E_{k}(F)$, and $p$ an interior point of the $k$-polyhedron $e$, that the space $Y(e, F)$ is an $n$-manifold. Moreover the canonical map $\pi: Y \rightarrow X$ is a homeomorphism of $Y(e, F)$ onto a neighborhood of Int $e$.

Consider now the canonical map $\pi: Y \rightarrow X$. The hypothesis that each $F \in \mathscr{F}$ contains an $r$-ball easily implies that the $\pi$ image of each connected component of $Y$ can contain no limit points and that it must be all of $X$. It follows at once that $\pi$ is a covering map. Since $X$ is simply connected, $\pi$ is univalent on each connected component. Since $\mathscr{F}$ is a connected family, $Y$ is connected and $\pi$ is a homeomorphism of $Y$ onto $X$.

THEOREM 6.3.2. (I) Let $F$ be a smooth polyhedron in the Riemannian manifold $X$. Let $\Delta$ be a finite subset of the isometry group Isom $X$ and let $\Gamma$ denote the subgroup of Isom $X$ generated by 4 . Assume

(1) $\Delta=\Delta^{-1}$.

(2) There is a bijective map $\gamma \rightarrow e(\gamma)$ of $\Delta$ onto $E_{1}(F)$ satisfying $\gamma(F) \cap F=e(\gamma)$ for all $\gamma \in \Delta$.

Set $\mathscr{N}=\{(\gamma F, \gamma \delta F) ; \gamma \in D\}, \mathscr{F}=\Gamma F$. Then $\mathscr{F}$ is a connected abutted family of polyhedra with adjacency $\mathscr{N}$. Moreover $\Gamma$ operates discontinuously on the joined $\mathscr{F}$-space $Y$.

(II) If, in addition, $\mathscr{F}$ satisfies the codimension 2 condition, (CD2) and $X$ is simply connected, then

(1) $\Gamma$ is a discrete subgroup of Isom $X$.

(2) Let $\operatorname{Aut}_{\Gamma} F$ denote the stabilizer of $F$ in $\Gamma$. Then a fundamental domain for Aut $F$ in $F$ is a fundamental domain for $\Gamma$ in $X$ (i.e., $F$ is a fundamental domain $\bmod \operatorname{Aut}_{F} F$ ).

Proof. That $\mathscr{F}$ is a connected abutted smooth family of polyhedra with adjacency $\mathfrak{h}$ follows directly from definitions. Clearly $\mathscr{F}$ yields a decomposition of the space $Y$ into polyhedra with disjoint interiors. Consider the action of $\Gamma$ on $Y$. It follows at 
once that $\Gamma$ operates discontinuously on $Y$, since it permutes the interiors of the polyhedra of $\mathscr{F}$. Under the additional hypotheses in (II), we can apply Theorem 6.3 .1 to conclude that $\pi: Y \rightarrow X$ is a homeomorphism. Hence $\Gamma$ is discontinuous on $X$.

REMARK. Let $\Gamma$ be a discontinuous group of isometries on a Riemannian space such as $C h^{n}$. Let $p_{0} \in X$, and set

$$
F=\left\{x \in X ; d\left(x, p_{0}\right) \leqq d\left(x, \Gamma p_{0}\right)\right\} .
$$

Then clearly $X=\Gamma F$. Also, for all $\gamma \in \Gamma$ with $\gamma p_{0} \neq p_{0}$, the interiors of $\gamma F$ and $F$ are disjoint. $F$ is called a "Dirichlet" fundamental domain" for $\Gamma$. For any $k$, let $E_{k}(F)$ denote the set of codimension $k$ faces of $F$. For each $e \in E_{1}(F)$, let $T(e)$ denote an element $\gamma^{-1}$ of $\Gamma$ such that

$$
e \subset\left\{x \in X ; d\left(x, p_{0}\right)=d\left(\gamma x, p_{0}\right)\right\} .
$$

Set $\Delta=T\left(E_{1}(F)\right)$. Then $T: E_{1}(F) \rightarrow \Delta$ is a bijective map and $\Delta=\Delta^{-1}$. Moreover, $F$ satisfies the condition

$$
T(e) F \cap F=e \text { for all } e \in E_{1}(F),
$$

and also the codimension-two condition (CD2). Thus the converse of Theorem 6.2 is valid, so that (CD1) and (CD2) are necessary conditions for a Dirichlet fundamental domain.

THEOREM 6.3.3. We continue the notation and hypotheses of Theorem 6.3.2 I and II. A presentation for the group $\Gamma$ is given by the generators $\triangle$ with the following relations:

For each $e \in E_{2}(F)$, choose $e_{1} \in E_{1}(F)$ with $e \subset e_{1}$ and let $r_{e}$ denote the word of shortest positive length $\gamma_{1} \gamma_{2} \cdots \gamma_{n}\left(\gamma_{i} \in \Delta\right)$ such that

(i) $e \subset \gamma_{1} \gamma_{2} \cdots \gamma_{i} F \cap \gamma_{1} \gamma_{2} \cdots \gamma_{i+1} F \in E_{1}\left(\gamma_{1} \cdots \gamma_{i} F\right)(i=1, \cdots, n-1)$.

(ii) $F \cap \gamma_{1} F=e_{1}$.

(iii) $F \cap \gamma_{1} \gamma_{2} \cdots \gamma_{n} F$ has a nonempty interior.

Set $R_{2}=\left\{r_{e} ; e \in E_{2}(F)\right\}$. (By condition (CD2), the words of $R_{2}$ yield elements of $\operatorname{Aut}_{\Gamma} F$. Set $R_{F}=$ \{relations among words of $R_{2}$ as elements of $\mathrm{Aut}_{\Gamma} F$ \}. Then

(a) $R_{2}$ generates $\mathrm{Aut}_{\Gamma} F$.

(b) $\left(\Delta, R_{F}\right)$ is a presentation for $\Gamma$.

Proof. Let $\mathscr{G}$ denote the free group generated by the elements of $\Delta$, let $R$ denote the kernel of the canonical homomorphism of $\mathscr{G}$ onto $\Gamma$ and let $R^{\prime}$ denote the preimage in $\mathscr{G}$ of $\operatorname{Aut}_{\Gamma} F$. One defines a homomorphism of $R^{\prime}$ into the fundamental group of $X-\Gamma E_{2}(F)$

${ }^{1}$ (Also known as Poincaré or normal fundamental domain.) 
as follows. Choose a base point $P_{0}$ interior to $F$ and a point $p_{r} \in$ $\gamma F$ for each $\gamma \in \Gamma$. For each $\gamma \in \Delta$, choose a path in $F \cup \gamma F$ from $p_{0}$ to $p_{\gamma}$ which does not meet any codimension 2-face of $F$ or $\gamma F$. To each element in $w$ in $\mathscr{G}$ there corresponds a path $\varphi(w)$ in $\Gamma F-\Gamma E_{2}(F)=X-\Gamma E_{2}(F)$ with initial point $p_{0}$; the path $\varphi(w)$ is a closed path if and only if $w \in R^{\prime}$. Moreover $\varphi\left(w_{1} w_{2}\right)=\varphi\left(w_{1}\right) \cdot \varphi\left(w_{2}\right)$. The resulting map $\theta: R^{\prime} \rightarrow \pi_{1}\left(X-\Gamma E_{2}(F)\right)$ is a homomorphism.

Inasmuch as $X$ is simply connected, $\pi_{1}\left(X-\Gamma E_{2}(F)\right)$ is generated by $\Gamma$-conjugates of closed paths linking $E_{2}(F)$, and therefore $\left\{\theta\left(r_{e}\right)\right.$; $e \in E_{2}(F)$ \} generates $\pi_{1}\left(X-\Gamma E_{2}(F)\right)$. It is easy to see that for $w \in$ $R^{\prime}$, any homotopy of $\theta(w)$ in $X-\Gamma E_{2}(F)$ leads to a path $\theta\left(w^{\prime}\right)$ where $w^{\prime}$ is obtained from $w$ by successive substitution of subwords $x z$ for $x y y^{-1} z(x, y, z \in \Delta)$, and vice-versa. It follows at once that the kernel of $\theta$ is (1) and $\theta$ is an isomorphism. In particular, $R_{2}=\left\{r_{e} ; e \in\right.$ $E_{2}(F)$ \} generates $R^{\prime}$. This proves (a).

Let $R_{F^{\prime}}$ denote the normal subgroup of $R^{\prime}$ generated by words in $R_{2}$ that represent 1 in $\operatorname{Aut}_{\Gamma} F$. Then $R_{F}$ represents 1 in $\Gamma$ and it is clear that $R_{F}=R$. Hence $\left(\Delta, R_{F}\right)$ is a presentation of $\Gamma$.

\subsection{Branching and complex analytic joined spaces.}

The foregoing results provide a criterion for deciding which of the subgroups of $U(2,1)$ presented in $\S 9$ are discrete. We shall require a generalization of the above discussion in order to treat the action of some nondiscrete subgroups of $U(2,1)$. The results of the rest of this section will not be required until $\S 18.3$.

Proposition 6.4.1. Let $\mathscr{F}$ be a connected abutted smooth family of compact polyhedra in the topological n-manifold $X$. Assume also that for any $F \in \mathscr{F}$ and $e \in E_{k}(F)$ the joined $\mathscr{F}_{e}\left(S_{e}\right)$ space is an $(k-1)$-sphere $(k=1, \cdots, n)$ (which is a finite branched over of $\left.S_{e}\right)$. Then the joined $\mathscr{F}$-space $Y$ is a topological n-manifold and $\mathscr{F}$ gives a polyhedral decomposition with disjoint interiors of $Y$.

Proof. This proposition follows directly from definitions. As asserted above, $Y(e, F)$ is a neighborhood of $\eta($ Int $e, F)$ in $Y$. By the hypothesis on $\mathscr{F}_{e}\left(S_{e}\right)$ and the smoothness of $\mathscr{F}$, Int $Y(e, F)$ is an $n$-manifold for every $k$-face $e(k=1,2, \cdots, n)$. It follows at once that $Y$ is an $n$-manifold. That $\mathscr{F}$ gives a polyhedral decomposition of $Y$ with disjoint interiors is obvious.

DEFINITION. Let $\mathscr{F}$ be an abutted smooth family on a space $X$. We say that $\mathscr{F}$ satisfies condition $\mathrm{BR}$ if 
(BR)

for all $F \in \mathscr{F}$ and $e \in E_{2}(\mathscr{F}), S(e, F)$, the joined ${ }_{e, F}\left(S_{e}\right)$ space is a circle.

If $\mathscr{F}^{-}$satisfies condition $\mathrm{BR}$, then the canonical map of $S(e, F)$ onto $S_{e}$ is an even covering map for every codimension 2 -face $e$; we denote the degree of this map by $\beta(e)$. We call $\beta(e)$ the branching order of $\mathscr{F}$ around $e$. We call a codimension ${ }_{R}$ 2-face $e$ branching if $\beta(e)>1$. Set

$$
B_{2}(\mathscr{F})=\{e ; \beta(e)>1\} .
$$

For any face $e \in E_{k}(F)$, where $F \in \mathscr{F}$, set $B^{2}(e)=\left\{e^{\prime} ; e^{\prime} \in E_{2}\left(F^{\prime}\right)\right.$ with $\left.F^{\prime} \in \mathscr{F}_{e, F}, \beta\left(e^{\prime}\right)>1\right\}$. Thus $B^{2}(e) \subset B_{2}(\mathscr{F})$ and $e \subset e^{\prime}$ for all $e^{\prime} \in B^{2}(e)$.

Proposition 6.4.2. Let $X$ be a complex analytic manifold of $n$ complex dimensions. Let $\mathscr{F}$ be an abutted smooth family of polyhedra on $X$, which satisfies condition BR. Assume:

(1) Each codimension ${ }_{R}$ 2-face $e$ such that $\beta(e)>1$ lies on $a$ hypersurface of $\boldsymbol{C}$-codimension 1.

(2) For any s branching codimension ${ }_{R} 2$-faces $\left\{e_{1}, \cdots, e_{s}\right\}$ whose hypersurfaces are distinct, $e_{1} \cap \cdots \cap e_{s}$ is either empty or has dimen$\operatorname{sion} 2 n-2 s$.

(3) Let $e \in E_{k}(F)$ with $F \in \mathscr{F}$, and let $e_{1}, \cdots, e_{s}$ be the distinct elements of $B^{2}(e)$ (thus $\left.k \geqq 2 s\right)$. Then for any compact subset $K \subset$ $e$, there exists an admissible complex analytic coordinate system $z=\left(z_{1}, \cdots, z_{n}\right)$ in a neighborhood $U$ in $X$ and a neighborhood $W$ of $\eta(K, F)$ in $Y(e, F)$ so that $U \cap e_{\imath} \subset\left\{z ; z_{i}=0\right\}, K \subset U=\pi(W)$, and the restriction to $W$ of the canonical map of $Y(e, F)$ into $X$ is equivalent to the $\operatorname{map}\left(w_{1}, \cdots, w_{n}\right) \rightarrow\left(z_{1}, \cdots, z_{n}\right)$ given by

$$
\begin{array}{ll}
z_{i}=w_{i}^{\beta\left(e_{\imath}\right)} & (i=1, \cdots, s) \\
z_{\imath}=w_{i} & (i=s+1, \cdots, n) .
\end{array}
$$

Then the joined $\mathscr{F}$-space has the structure of a complex analytic n-manifold such that the canonical map into $X$ is holomorphic.

Proof. For any face $e$ such that $\beta\left(e^{\prime}\right)=1$ for all codimension 2 -faces $e^{\prime}$ containing $e$, the (codim 2) condition of Theorem 6.3.1 is satisfied, and we can argue just as we did there that the canonical map of $Y(e, F)$ into $X$ is a homeomorphism onto a neighborhood in $X$; we endow such $Y(e, F)$ with the structure pulled back from $X$.

For faces $e$ lying in $s$ branching codimension 2-faces with branching orders $m_{1}, m_{2}, \cdots, m_{s}$ the hypotheses give us the structure of a complex analytic manifold on $Y(e, F)$, such that the canonical map of $Y(e, F)$ into $X$ has degree $m_{1} m_{2} \cdots m_{s}$. Putting together 
these complex analytic structures from the various $Y(e, F)$, it can be verified directly that we get the desired complex analytic structure on $Y$.

REMARK. Let $X$ be a complex analytic manifold. Let $\mathscr{F}$ be an abutted smooth family of compact polyhedra on $X$ which satisfy condition BR. Hypothesis (3) of Proposition 6.5 follows from the apparently weaker hypotheses in which we restrict the face $e \in$ $E_{k}(F)$ to satisfy the condition: $B^{2}(e)$ is a maximal subset among $\left\{B^{2}\left(e^{\prime}\right)\right.$; all faces $\left.e^{\prime}\right\}$. The verification of this observation comes from straightforwardly studying the situation of $Y\left(e^{\prime}, \mathscr{F}\right)$ in $Y(e, \mathscr{F})$ when $e \subset e^{\prime}$.

\subsection{Polyhedral $\Gamma$-spaces $Y$ and the $\Gamma$-cover $Y^{\# \text {. }}$}

A polyhedral $\Gamma$-space $Y$ is a topological space which is covered by a family $\mathscr{F}$ of polyhedra with disjoint interiors, together with a group $I$ of transformations of $Y$ which permute the polyhedra of $\mathscr{F}$. The polyhedral $\Gamma$-space is called a joined $\Gamma$-space if

(1) $\Gamma$ acts transitively on $\mathscr{F}$ i.e.. $\mathscr{F}=\Gamma F$ where $F \in \mathscr{F}$.

(2) There is an injective map $E_{1}(F) \rightarrow \Gamma$ satisfying

(1) $\Delta=\Delta^{-1}$ where $\Delta=T\left(E_{1}(F)\right)$,

(2) $T(e) F \cap F=e$ for all $e \in E_{1}(F)$.

(3) $\Delta$ generates the group $\Gamma$.

We define the subset of $\mathscr{F} \times \mathscr{F}$ :

$$
\mathscr{N}=\{(\gamma F, \gamma \delta F), \gamma \in \Gamma, \delta \in \Delta\} .
$$

Then $\mathscr{F}$ is clearly an abutted family of polyhedra on $Y$ with adjacency $\mathscr{N}$, and the joined $\mathscr{F}$-space may be identified with $Y$.

Let $\Gamma_{F}$ denote the stabilizer in $\Gamma$ of the polyhedron $F$. We can define a joined $\Gamma$-space $Y^{\sharp}$ covered by a family $\mathscr{F}^{\sharp}$ of polyhedra and a $\Gamma$-map $\pi^{\sharp}: Y^{\sharp} \rightarrow Y$ such that $\pi^{\sharp} \mathscr{F}^{\sharp}=\mathscr{F}$ and $\Gamma_{F \sharp}=(1)$ for $F^{\sharp} \in \mathscr{F}^{\sharp}$. Namely, fix $F \in \mathscr{F}$, let $\Gamma$ operate on the topological space $\Gamma \times F$ via left multiplication on the first factor. Set

$$
F^{\#}=(1, F)
$$

and define the $\Gamma$-stable equivalence relation on $\Gamma \times F$ generated by

$$
(1, y)=\left(\delta, \delta^{-1}(y)\right) \text { for all } y \in e, \delta=T(e)
$$

where $e$ varies over $E_{1}(F)$. Let $Y^{\#}$ denote the quotient topological space $\Gamma \times F \bmod \equiv$ and $\mathscr{F}^{\sharp}=\left\{\gamma F^{\sharp} ; \gamma \in \Gamma\right\}$. The map $\pi^{\sharp}:(\gamma, y) \rightarrow \gamma y$ of $\Gamma \times F \rightarrow Y$ induces a well-defined continuous $\Gamma$ map of $Y^{\sharp}$ to $Y$. Moreover the stabilizer $\Gamma_{F^{\sharp}}=(1)$; for if $(1, y)$ is an interior point of 
$F^{*}$ and $\gamma \in \Gamma_{F^{*}}$, then $\gamma(1, y) \equiv\left(1, y^{\prime}\right)$ with $y, y^{\prime}$ interior points of $F$. Since the only identifications on interior points of $(1, F)$ come from equality, we conclude that $\gamma(1, y)=\left(1, y^{\prime}\right)$. Consequently $(\gamma, y)=$ $\left(1, y^{\prime}\right)$ and $\gamma=1$.

Definition. $\quad Y^{\ddagger}$ is called the $\Gamma$-cover of the joined $\Gamma$-space $Y$.

On the associated family of polydedra $\mathscr{F}^{\sharp}, \Gamma$ operates simply transitively, since $\Gamma_{F \sharp}=(1)$.

Warning. Even if $Y$ is a manifold, $Y^{\sharp}$ need not be. However, $Y^{\#}$ is connected, locally connected, and locally simply connected if $Y$ is a manifold.

\subsection{The stabilizer of a face in a joined $\Gamma$-space.}

Let $Y$ be a joined $\Gamma$-space, let $\mathscr{F}$ denote the associated abutted family of polyhedra on which $\Gamma$ operates transitively, and let $\mathfrak{h}$ denote its adjacency. Let $F \in \mathscr{F}$ and $e \in E_{k}(F)$. In keeping with previous notation, set

$$
\begin{aligned}
& F_{e}=\cup\left\{\operatorname{Int} f ; f \in E_{k}(F)(k=0,1,2, \cdots) \text { and } e \subset f\right\} \\
& \mathscr{F}_{e}=\left\{F_{e} ; F \in \mathscr{F} \text { and } e \subset F\right\} \\
& \mathscr{F}_{e, F}=\text { the maximum connected subfamily of } \mathscr{F}_{e} \text { containing } F_{e} \\
& G(e, F)=\left\{\gamma \in \Gamma ;(\gamma F)_{e} \in \mathscr{F}_{e, F}\right\} \\
& \Gamma_{e, F^{*}}=\text { stabilizer of } e \text { and of } \mathscr{F}_{e, F} \text { in } \Gamma .
\end{aligned}
$$

Clearly $\Gamma_{(e, F)} \subset G(e, F), \Gamma_{(e, F)} G(e, F)=G(e, F)$, and $\left\{G(e, F) F_{e}\right\}=\mathscr{F}_{\left(e, F^{\prime}\right)}$. Tracing back definitions, one sees that

$$
\begin{gathered}
G(e, F)=\left\{\gamma \in \Gamma ; \gamma=\gamma_{1} \gamma_{2} \cdots \gamma_{m}, \gamma_{i} \in \Delta, e \in E_{k}\left(\gamma_{1} \gamma_{2} \cdots \gamma_{i} F\right),\right. \\
i=1,2, \cdots, m ; m=1,2\} .
\end{gathered}
$$

REMARK 1. If the underlying topological space $Y$ is a $n$-manifold as it is in the cases of interest here, then $\mathscr{F}_{e, F}=\mathscr{F}_{e}$. Theorem 6.3.2 (I) describes the situation out of which our joined $\Gamma$-spaces will arise.

The family $\mathscr{F}_{e}$ is an abutted family of polyhedra and we denote $\mathscr{N} \cap\left(\mathscr{F}_{e} \times \mathscr{F}_{e}\right)$ by $\mathscr{N}$ also; it is the adjacency of $\mathscr{F}_{e}$.

Proposition 6.6. Let $e \in E_{k}(F)(k=0,1,2, \cdots, n)$ and let $\Gamma^{\prime}$ be a subgroup of $\Gamma(e, F)$. If

$$
\mathscr{N}\left(\mathscr{N}\left(\mathscr{F}_{e}\right) \subset \Gamma^{\prime}\left(\mathscr{N}\left(F_{e}\right) \cup F_{e}\right)\right.
$$

then $\mathscr{F}_{e, F}=\Gamma^{\prime}\left(\mathscr{N}\left(F_{e}\right) \cup F_{e}\right)$ and $\Gamma_{\left(e, k^{\prime}\right)}=\Gamma^{\prime} S$ where $S=\{\gamma \in \Gamma ; \gamma F \in$ $\mathscr{N}(F), \gamma e=e\}$. 
Proof.

$$
\begin{aligned}
\mathscr{N}\left(\Gamma^{\prime}\left(\mathfrak{h}\left(F_{e}\right) \cup F_{e}\right)\right) & =\Gamma^{\prime}\left(\mathscr{N}\left(\mathscr{N}\left(F_{e}\right) \cup \mathscr{N}\left(F_{e}\right)\right)\right) \\
& \subset \Gamma^{\prime}\left(\mathscr{N}\left(F_{e}\right) \cup \Gamma^{\prime} F_{e}\right) \\
& \subset \Gamma^{\prime}\left(\mathscr{N}\left(F_{e}\right) \cup F_{e}\right) .
\end{aligned}
$$

Hence $\Gamma^{\prime}\left(\mathscr{N}\left(F_{e} \cup F_{e}\right)\right)$ is an open subfamily of $\mathscr{F}_{e, F}$; since it contains $F_{e}$ it contains the e-connected family $\mathscr{F}_{e, F}$. This proves the first assertion.

As mentioned above, $\mathscr{F}_{e, F}=G(e, F) F_{e}$ and $I_{\left(e, F^{\prime}\right)} G(e, F)=G(e, F)$. Let $\Gamma=\left\{\gamma \in \Gamma ; \gamma F_{e} \in \mathscr{N}\left(F_{e}\right) \cup F_{e}\right\}$. Then we have $G(e, F)=I^{\prime \prime} T$. Hence $\Gamma_{\left(e, F^{\prime}\right)}=\Gamma^{\prime}\left(T \cap \Gamma_{\left(e, F^{\prime}\right)}\right)$. Clearly $T \cap \Gamma_{\left(e, F^{\prime}\right)}=S$. Consequently $\Gamma_{\left(e, F^{\prime}\right)}=\Gamma^{\prime} S$.

REMARK 2. Set $F[e]=\left\{\gamma \in \Delta ; \gamma F_{e} \in \mathfrak{b}\left(F_{e}\right)\right\}, T^{\prime}=\{1\} \cup F[e]$, and let $S^{\prime}$ be any subset of $T^{\prime}$ such that $\Gamma^{\prime} S^{\prime}=\Gamma^{\prime} T^{\prime}$. Then $S=T^{\prime} \Gamma_{r}$, $\Gamma_{(e, F)}$ and therefore $\Gamma^{\prime} S=\Gamma^{\prime}\left(T^{\prime} \Gamma_{F} \cap \Gamma_{(e, F)}\right)=\Gamma^{\prime} T^{\prime} \Gamma_{F} \cap \Gamma_{\left(e, F^{\prime}\right)}=\Gamma^{\prime} S^{\prime} \Gamma_{F^{\prime}} \cap$ $\Gamma_{\left(e, F^{\prime}\right)}=\Gamma^{\prime}\left(S^{\prime} \Gamma_{F} \cap \Gamma_{(e, F)}\right)$.

REMARK 3. Set $S^{\#}=\left(F[e] \cap \Gamma_{(e, F)}\right) \cup\{1\}$. In the $\Gamma$-cover $Y^{\#}$ of the joined $\Gamma$-space $Y$, let $\mathscr{F}^{\#}$ and $\mathscr{N}^{\#}$ denote the associated abutted family and adjacency. For $F \in \mathscr{F}$ and $e \in E_{k}(F)$, set $F^{\sharp}=(1, F)$ and $e^{\sharp}=(1, e)$. Then $G\left(e^{\sharp}, F^{\sharp}\right)=G(e, F)$ and $\Gamma(e, F) G\left(e^{\sharp}, F^{\sharp}\right)=G\left(e^{\sharp}\right.$, $\left.F^{\sharp}\right)$ and $\Gamma_{\left(e \sharp, l^{\prime} \sharp\right)}=\Gamma^{\prime}\left(S^{\sharp} \cup\{1\}\right)$.

7. Fundamental domains for finite groups generated by $C$ reflections.

7.1. An algorithm for finding the faces of a fundamental domain in $\mathrm{Ch}^{2}$

Let $\Gamma_{12}$ be a finite group generated by two $C$-reflections $\left\{R_{e_{1}, p_{1}}\right.$, $\left.R_{e_{2}, p_{2}}\right\}$ in $C^{3}$ and preserving a hermitian form $H$ of type $(2,1)$. We follow the notation of $\S 3$ writing $V(H)$ or $V$ for $C^{3}$ with $H$ as inner product:

$$
\begin{aligned}
& \langle p, q\rangle=H(p, q) \quad \quad p, q \in V \\
& V^{-}=\{p \in V ;\langle p, p\rangle\langle 0\} \\
& C h^{2}=\pi\left(V^{-}\right), \quad X=C h^{2} .
\end{aligned}
$$

Fix a point $p_{0} \in C h^{2}$ fixed by no $\gamma \in \Gamma_{12}, \gamma \neq 1$. For any $\gamma \in \Gamma_{12}$, set

$$
\begin{aligned}
& \gamma^{+}=\left\{x \in X ; d\left(x, p_{0}\right) \leqq d\left(\gamma x, p_{0}\right)\right\} \\
& \hat{\gamma}=\left\{x \in X ; d\left(x, p_{0}\right)=d\left(\gamma x, p_{0}\right)\right\} \\
& F_{12}=\bigcap_{i \in l} \gamma^{+} .
\end{aligned}
$$


It is easy to see that

(1) $\Gamma_{12} F_{12}=X$

(2) $\gamma F_{12} \cap F_{12}$ has empty interior if $\gamma \neq 1$.

These two properties define a fundamental domain.

In order to determine whether the domains we encounter in $\S 9$ satisfy condition (CD1), we must explicitly know the faces of the fundamental domain $F_{12}$. Some of the groups under consideration have order 600 , and thus the explicit computation of (7.1.1) would be lengthy, even if we were merely seeking a fundamental domain for the action of $F_{12}$ in $C^{2}$.

Despite the length of the computation, it is clearly finite. Therefore a computing machine can be used to settle the question.

Let $p_{12}=\pi\left(e_{1}^{\perp} \cap e_{2}^{\perp}\right)$, then $p_{12}$ is the point in $\pi(V)$ fixed under $R_{e_{1}, p_{1}}$ and $R_{e_{2}, p_{2}}$. Inasmuch as $0 \leqq\left|\left\langle e_{1}, e_{2}\right\rangle\right|<1, e_{1}^{\perp} \cap e_{2}^{\perp} \subset V^{-} \cup\{0\}$ and thus $p_{12} \in \pi\left(V^{-}\right)=C h^{2}$. We call $p_{12}$ the apex of $F_{12}$ since $\gamma p_{12}=$ $p_{12}$ for all $\gamma \in \Gamma_{12}$ and thus $p_{12} \in \hat{\gamma}$ for all $\gamma \in \Gamma_{12}$. Let $S_{r}$ denote the boundary of the ball in $C h^{2}$ with center at $p_{12}$ and radius $r$. Then $S_{r}$ is $\Gamma$-stable. A machine can best be used to compute numbers. Thus, the machine can be programmed to compute the coordinates of the vertices of $S_{r} \cap F_{12}$ for any given value of $r$. If we select $r=d\left(p_{12}, p_{0}\right)$, then $p_{0} \in S_{r}$ and $S_{r} \cap F_{12}$ is a Dirichlet fundamental domain for $\Gamma_{12}$ acting on $S_{r}$ (with distance on $S_{r}$ defined by the ambient $\left.C h^{2}\right)$.

A machine can be programmed to find the vertices of $S_{r} \cap F_{12}$ as follows.

Set

$$
\begin{aligned}
& d=\inf \left\{d\left(\gamma p_{0}, p_{0}\right) ; \gamma \in \Gamma_{12}\right\} \\
& D_{0}=\left\{\gamma \in \Gamma_{12} ; d\left(\gamma p_{0}, p_{0}\right)<2 d\right\}
\end{aligned}
$$

(The 2 is merely a good empirical choice.) Let $T_{0}$ denote the set of distinct triplets of elements of $D_{0}$. In each distinct triplet $t=$ $\left(\gamma_{1}, \gamma_{2}, \gamma_{3}\right)$ of elements in $\Gamma$, set

$$
V_{t}=\hat{\gamma}_{1} \cap \hat{\gamma}_{2} \cap \hat{\gamma}_{3} \cap S_{r}
$$

Set $V_{0}=\cup\left\{V_{t} ; t \in T_{0}, V_{t}\right.$ is finite $\}$. For any $x \in X$, and for any subset $D \subset X$, set

$$
\begin{aligned}
& D^{\sharp}(x)=\left\{\gamma \in D ; d\left(\gamma x, p_{0}\right)=d\left(x, p_{0}\right)\right\} \\
& E(x)=\left\{\gamma \in \Gamma_{12} ; d\left(\gamma x, p_{0}\right)<d\left(x, p_{0}\right)\right\}
\end{aligned}
$$

and let $\sigma(x)$ denote an element of $\Gamma_{12}$ such that

$$
d\left(\sigma(x) x, p_{0}\right)=\inf \left\{d\left(\gamma x, p_{0}\right) ; \gamma \in E(x)\right\} .
$$

Set 


$$
\begin{aligned}
& V_{0}^{\sharp}=\left\{x \in V_{0} ; d\left(x, p_{0}\right) \leqq d\left(\gamma x, p_{0}\right) \text { for all } \gamma \in D_{0}\right\} \\
& D_{0}^{\ddagger}=\cup\left\{D_{0}^{\ddagger}(x) ; x \in V_{0}^{\ddagger}\right\} \\
& S_{0}=\left\{x \in V_{0}^{\ddagger} ; E(x) \text { is not empty }\right\} \\
& E_{0}=\left\{\sigma(x) ; x \in S_{0}\right\} \\
& D_{1}=D_{0}^{\ddagger} \cup E_{0} .
\end{aligned}
$$

Recursively, define

$$
\begin{aligned}
& D_{i+1}=D_{i}^{*} \cup E_{i} \\
& T_{i+1}=\text { the set of distinct triplets of elements of } D_{i+1} \\
& V_{i+1}=\bigcup\left\{V_{t} ; t \in T_{i+1}, V_{t} \text { is finite }\right\} \\
& V_{i+1}^{*}=\left\{x \in V_{i+1} ; d\left(x, p_{0}\right) \leqq d\left(\gamma x, p_{0}\right) \text { for all } x \in D_{i+1}\right\} \\
& D_{i+1}^{*}=\bigcup\left\{D_{i+1}^{*}(x) ; x \in V_{i+1}^{\#}\right\} \\
& S_{i+1}=\left\{x \in V_{i+1}^{*} ; E(x) \text { is not empty }\right\} \\
& E_{i+1}=\left\{\sigma(x), x \in S_{i+1}\right\} .
\end{aligned}
$$

Each element of $V_{i}^{*}$ is a vertex of

$$
\cap\left\{\gamma^{+} ; \gamma \in D_{i}^{\sharp}\right\} \text {. }
$$

Each $x \in V_{i}^{\sharp}$ with $E(x)$ not empty is cut off by $\hat{\gamma}$ for $\gamma \in E(x)(\sigma(x)$ makes a deepest cut) and disappears from $V_{i+1}^{\#}$. After a finite number of cuts, the process stops. At the final stage, no more vertices of $V_{n}^{\sharp}$ can be cut off and $V_{n}^{\sharp}$ is the set of vertices of $F_{12} \cap$ $S_{r}$. Let $\Delta_{12}$ denote the set of all $\gamma \in D_{n}^{\sharp}$ such that $\hat{\gamma}$ contains at least three points of $V_{n}^{\sharp}$. Then $\left\{\hat{\gamma} ; \gamma \in \Delta_{12}\right\}$ yield the 3 -faces of $F_{12}$ which meet the sphere $S_{r}$.

REMARK. It turns out in most cases that $\Delta_{12} \subset D_{0}$.

\subsection{Enumeration of $\Gamma_{12}$.}

In programming the algorithm of $\S 7.1$ for the computation of the faces of $F_{12} \cap S_{r}$, it is convenient to have a simple way of enumerating the finite group $\Gamma_{12}$, for repeatedly one has to compute

$$
\left\{d\left(\gamma x, p_{0}\right) ; \gamma \in \Gamma_{12}\right\} \text {. }
$$

In $\S 2.1$, we remarked that the canonical map of $C^{2}-0$ to the complex projective line gives a representation $\rho$ of $\Gamma_{12}$ in $\operatorname{SO}(3, \boldsymbol{R})$ the group of notations of the standard 2-sphere $S^{2}$. The kernel of $\rho$ is the center $Z$ of $\Gamma_{12}$ whose generator and order is given by (2.2.3) and (2.2.4).

For the groups $\Gamma_{12}$ with Coxeter diagram 


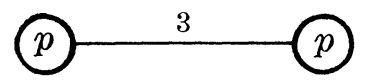

the group $\rho\left(\Gamma_{12}\right)$ is the group of rotational symmetries of the regular tetahedron, cube, and duodecahedron respectively according as $p=$ 3, 4, and 5. Each generating $C$-reflection $R_{i}$ corresponds to a rotation of a face of the regular polyhedron $\mathscr{R}(p)$. Let $\Gamma_{1}=\left\{\left\{R_{1}\right\}\right\}$, the subgroup generated by $R_{1}$.

Each element of $\Gamma_{12}$ can be expressed as a product $\gamma \cdot R_{1}^{n} z^{m}$ where $\gamma$ is a representative of a coset of $\Gamma_{12} / \Gamma_{1} Z$, and $z$ is a generator of $Z$. In turn $\Gamma_{1} Z$ is the stabilizer in $\Gamma$ of the face of $\mathscr{R}(p)$ stabilized by $\Gamma_{1}$. Hence $\sharp \Gamma_{12} / \Gamma_{1} Z=4,6,12$ according as $p=3,4,5$. Explicit representatives are indicated in Figures $7.2 \mathrm{a}, \mathrm{b}$, c: the face labeled 1 is stabilized by $R_{1}$ and the face labeled $\gamma$ is the image of the face 1 under $\gamma$. Thus the computer runs through $\Gamma_{12}$ by running

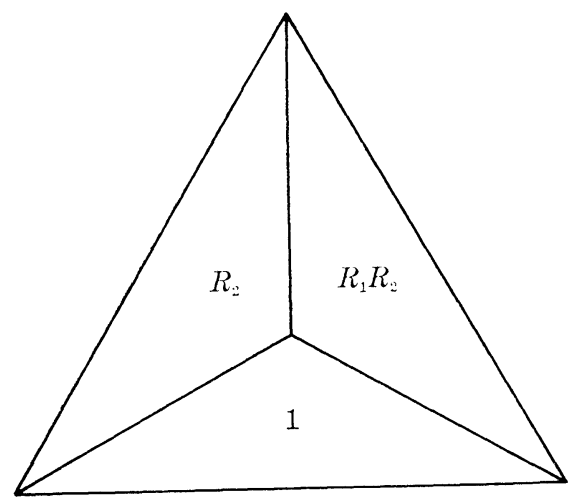

Figure 7.2a. $\quad p=3$ : Representatives for $\Gamma_{12} / \Gamma_{1} Z$

The bottom face represents the coset $R_{1}^{2} R_{2} \Gamma_{1} Z$.

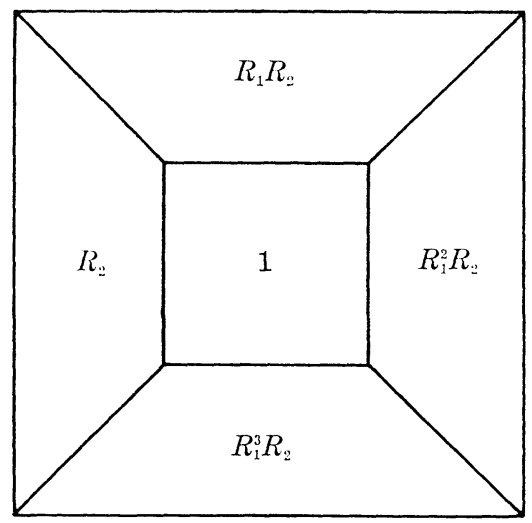

Figure 7.2b. $\quad p=4$ : Representatives for $\Gamma_{12} / \Gamma_{1} Z$

The bottom face represents the coset $R_{2}^{2} \Gamma_{1} Z$. 


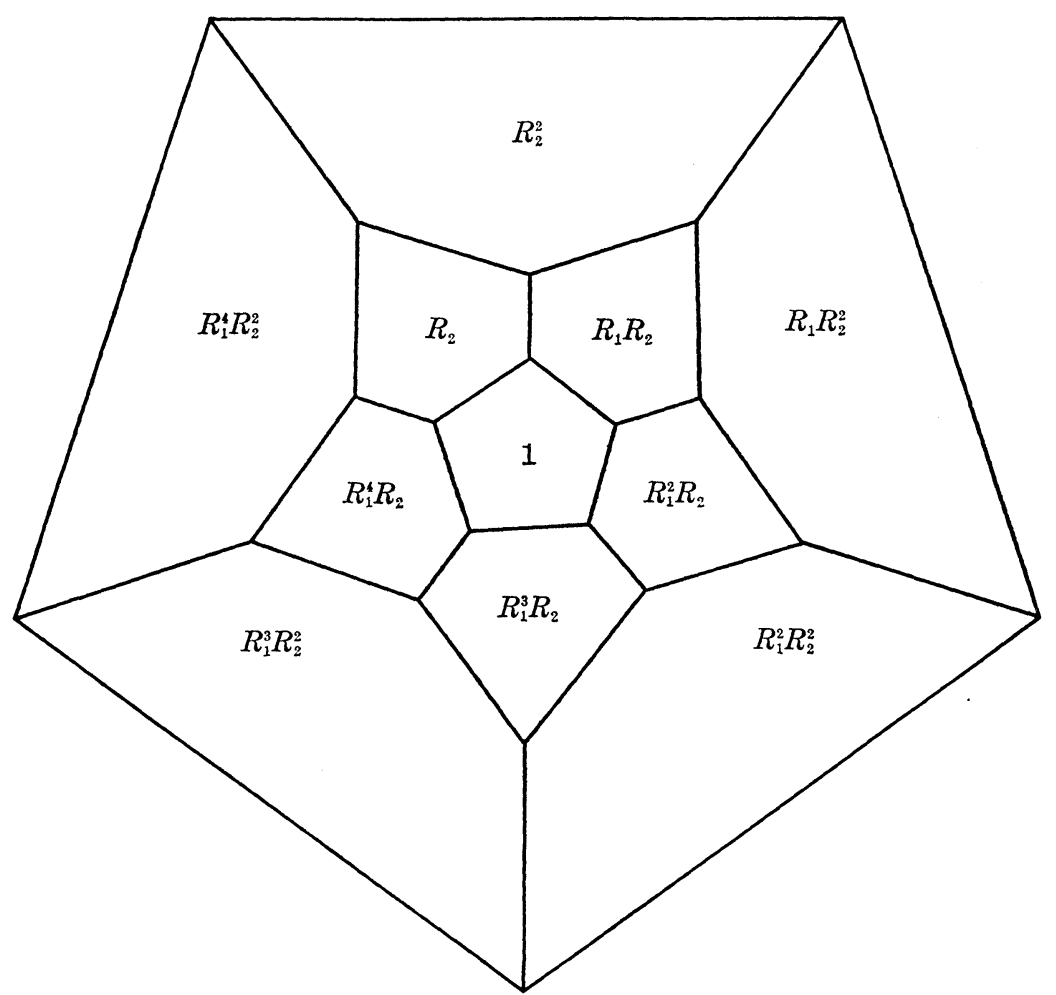

FIGURE 7.2c. $p=5$ : Representatives for $\Gamma_{12} / \Gamma_{1} Z$

The bottom face represents the coset $R_{2}^{-1} R_{1}^{2} R_{2}^{2} \Gamma_{1} Z$.

through the 3 -fold product $\left(\Gamma_{12} / \Gamma_{1} Z, \Gamma_{1}, Z\right)$ of orders

$$
\begin{aligned}
& \frac{12}{6-p} \times p \times \frac{2 p}{6-p}=24\left(\frac{p}{6-p}\right)^{2} \text { i.e., } \\
& 24,96,600 \text { according as } p=3,4,5 .
\end{aligned}
$$

Similarly, if $\Gamma^{\prime}$ has the Coxeter diagram

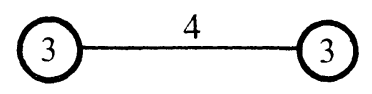

then the representatives of $\Gamma^{\prime} / \Gamma_{1} Z$ can be read off the faces of the regular octohedron. Note that $\# \Gamma^{\prime}=72$ by (2.2.5).

In $\S 10.1$, we give an explicit description of the Dirichlet fundamental domain $F_{12}$ for $\Gamma_{12}$ on $C h^{2}$ : its 3-dimensional faces lie in $\left\{\hat{\gamma}_{\text {; }}\right.$ $\left.\gamma \in \Delta_{12}\right\}$, where

$$
\Delta_{12}=\left\{R_{i}^{ \pm 1},\left(R_{i} R_{j}\right)^{ \pm 1},\left(R_{i} R_{j} R_{i}\right)^{ \pm 1}, i \neq j, i, j=1,2,3\right\},
$$

a set of 10 elements. By contrast the group $\Gamma^{\prime}$ has as Dirichlet fundamental domain a much more complicated region having 24 faces. 


\section{Solving a system of four equations of degree 2 .}

In $\S 7.1$, we described an algorithm for finding a fundamental domain for a finite group generated by two $C$-reflections in $C h^{2}$. That algorithm can be executed readily by a computer, provided that there is a program for computing the intersection of surfaces such as $\hat{\gamma}_{1} \cap \hat{\gamma}_{2} \cap \hat{\gamma}_{3} \cap S_{r}$. In $\S 9$, one deals with intersections $\hat{\gamma}_{1} \cap \hat{\gamma}_{2} \cap \hat{\gamma}_{3} \cap \hat{\gamma}_{4}$. If we introduce nonhomogeneous coordinates $y_{1}, y_{2}$ on $C h^{2}$ and express the equation of $\hat{\gamma}$ or of $S_{r}$ in terms of $\operatorname{Re} y_{1}, \operatorname{Im} y_{1}, \operatorname{Re} y_{2}, \operatorname{Im} y_{2}$ the equations turn out to be real polynomial equations of degree 2. Strange to say, as of 1978 there seemed to be no reliable program in the computation centers' bibliography which could provide all solutions of four such simultaneous equations. Accordingly the implementation of the algorithm necessitates elimination of variables and reduction to a polynomial equation of degree six in a single variable. We sketch the solution.

For any $\gamma \in U(H)$, the surface $\hat{\gamma}$ is by definition $\left\{x \in C h^{2} ; d\left(\gamma x, p_{0}\right)=\right.$ $\left.d\left(x, p_{0}\right)\right\}$. For convenience, we denote a point $x \in C^{3}-\{0\}$ and $\pi(x) \in$ $\boldsymbol{C} p^{2}$ by the same letter $x$. Thus the preimage of $\hat{\gamma}$ in $\boldsymbol{C}^{3}$ satisfies (in view of $\langle\gamma x, \gamma x\rangle=\langle x, x\rangle$ ):

$$
\left|\left\langle\gamma x, p_{0}\right\rangle\right|=\left|\left\langle x, p_{0}\right\rangle\right|
$$

where $\gamma e_{i}=\sum_{j=1}^{3} \gamma_{j i} e_{j}$,

$$
x=x_{1} e_{1}+x_{2} e_{2}+x_{3} e_{3} \text {, and } p_{0}
$$

is selected so that $\left\langle e_{1}, p_{0}\right\rangle=\left\langle e_{2}, p_{0}\right\rangle=\left\langle e_{3}, p_{0}\right\rangle=h$ and $\left\langle p_{0}, p_{0}\right\rangle\langle 0$. Thus (8.1) is equivalent to

$$
\begin{aligned}
& \left|(\gamma x)_{1}+(\gamma x)_{2}+(\gamma x)_{3}\right|=\left|x_{1}+x_{2}+x_{3}\right| \\
& y_{i}=\frac{x_{i}}{x_{1}+x_{2}+x_{3}} \quad(i=1,2,3) .
\end{aligned}
$$

Then $y_{1}+y_{2}+y_{3}=1$ and $y_{1}, y_{2}$ form a nonhomogeneous coordinate system on $C P^{2}$. Thus (8.2) is equivalent to

$$
\left|\alpha_{1} y_{1}+\alpha_{2} y_{2}+\alpha_{3} y_{3}\right|=1, \quad \alpha_{i}=\gamma_{1 i}+\gamma_{2 i}+\gamma_{3 i}
$$

or

$$
\begin{aligned}
& \left|\left(\alpha_{1}-\alpha_{3}\right) y_{1}+\left(\alpha_{2}-\alpha_{3}\right) y_{2}+\alpha_{3}\right|=1 \\
& \left|a y_{1}+b y_{2}+c\right|=1
\end{aligned}
$$

The equation of a sphere with center at a point $p$

$$
S_{r}=\left\{x \in C h^{2} ; d(x, p)=r\right\}
$$

and radius $r$ is given by 


$$
\begin{gathered}
\cosh ^{-1} \frac{|\langle x, p\rangle|}{(\langle x, x\rangle\langle p, p\rangle)^{1 / 2}}=r \\
|\langle x, p\rangle|^{2}=(\cosh r)^{2}\langle p, p\rangle\langle x, x\rangle=k\langle x, x\rangle, k<0 \\
\left|\alpha_{1} x_{1}+\alpha_{2} x_{2}+\alpha_{3} x_{3}\right|^{2}=k \sum_{i, j=1}^{3} h_{i j} x_{i} \bar{x}_{j} .
\end{gathered}
$$

Dividing both sides by $\left|x_{1}+x_{2}+x_{3}\right|^{2}$ and collecting terms, one gets

$$
2 \operatorname{Re}\left(g_{1} y_{1}+g_{2} y_{2}+g_{5} y_{1} \bar{y}_{2}\right)+g_{3} y_{1} \bar{y}_{1}+g_{4} y_{2} \bar{y}_{2}+g_{6}=0
$$

where $g_{3}, g_{4}, g_{6}$ are real.

Given two distinct surfaces of type (8.3):

$$
\begin{aligned}
& \left|a_{1} y_{1}+b_{1} y_{2}+c_{1}\right|=1 \\
& \left|a_{2} y_{1}+b_{2} y_{2}+c_{2}\right|=1
\end{aligned}
$$

Set

$$
\begin{aligned}
& v_{1}=a_{1} y_{1}+b_{1} y_{2} \\
& v_{2}=a_{2} y_{1}+b_{2} y_{2} .
\end{aligned}
$$

If $v_{2}=c v_{1}$, then (8.5) is equivalent to $\left|v_{1}+c_{1}\right|=1,\left|v_{1}+c_{2} / c\right|=$ $1 /|c|$, and these can be solved in the $v_{1}$-plane to give 2 solutions for $v_{1}$; only one solution is in $C h^{2}$ by Lemma 3.4.1. The solution of a system of 3 equations of type (8.3) with a fourth of type (8.3) or (8.4) then reduces to intersecting these surfaces with the $\boldsymbol{C}$-line $v_{1}=$ constant. The problem reduces to solving a quadratic equation in one real variable. Suppose therefore that $v_{1}$ and $v_{2}$ are independent. Then (8.5) is equivalent to

$$
\left|v_{1}\right|=1,\left|v_{2}\right|=1 \text {. }
$$

Expressing a third equation of type (8.3) in terms of $v_{1}, v_{2}$ yields

$$
\left|a_{3} v_{1}+b_{3} v_{2}+c_{3}\right|=1
$$

Squaring both sides, we get using (8.6)

$$
2 \operatorname{Re} v_{1}\left(b_{3} v_{2}+c_{3}\right)+d v_{2}+e=0
$$

with $e$ real.

Thus we need only solve the system

$$
\begin{aligned}
\left|v_{1}\right| & =1 \\
\left|v_{2}\right| & =1 \\
& v_{1}\left(\bar{b}_{1} \bar{v}_{2}+\bar{c}_{1}\right)+\bar{v}_{1}\left(b_{1} v_{2}+c_{1}\right)+d_{1} v_{2}+\bar{d}_{1} \bar{v}_{2}+e_{1}=0 \\
& v_{1}\left(\bar{b}_{2} \bar{v}_{2}+\bar{c}_{2}\right)+\bar{v}_{1}\left(b_{2} v_{2}+c_{2}\right)+d_{2} v_{2}+\bar{d}_{2} \bar{v}_{2}+e_{2}=0
\end{aligned}
$$


with $e_{1}, e_{2}$ real.

Elimination of $\bar{v}_{1}$ from these last two equations ${ }^{2}$ yields

$$
v_{1}\left(G_{1} v_{2}-\bar{G}_{1} \bar{v}_{2}+G_{3}\right)-F_{1} v_{2}^{2}-F_{2} v_{2}-F_{3} \bar{v}_{2}-F_{4}=0,
$$

and solving for $v_{1}$

$$
v_{1}=\frac{F_{1} v_{2}^{2}+F_{2} v_{2}+F_{3} \bar{v}_{2}+F_{4}}{G_{1} v_{2}-\bar{G}_{1} \bar{v}_{2}+G_{3}}
$$

Since $\left|v_{1}\right|=1$, we get

$$
\left|F_{1} v_{2}^{2}+F_{2} v_{2}+F_{3} \bar{v}_{2}+F_{4}\right|=\left|G_{1} v_{2}-\bar{G}_{1} \bar{v}_{2}+G_{3}\right| \text {. }
$$

Squaring both sides yields an equation

$$
2 \operatorname{Re}\left(P_{1} v_{2}^{3}+P_{2} v_{2}^{2}+P_{3} v_{2}\right)+P_{4}=0
$$

with $P_{4}$ real. Multiplying the above equation by $2 \operatorname{Re}\left(\bar{P}_{1} v_{2}^{3}+\bar{P}_{2} v_{2}^{2}+\right.$ $\left.\bar{P}_{3} v_{2}\right)+P_{4}$, one obtains an equation of the form

$$
2 \operatorname{Re}\left(Q_{1} v_{2}^{5}+Q_{2} v_{2}^{5}+Q_{3} v_{2}^{4}+\cdots+Q_{6} v_{2}+Q_{7}\right)=0
$$

with $Q_{i} \in R$ for $i=1, \cdots, 7$. Set

$$
v_{2}=z+i w \text {. }
$$

Since $\left|v_{2}\right|=1$, Re $v^{n}$ is a polynomial in $z$ and thus (8.8) becomes

$$
R_{1} z^{6}+R_{2} z^{5}+R_{3} z^{4}+R_{4} z^{3}+R_{5} z^{2}+R_{6} z+R_{\tau}=0
$$

with $R_{i}$ real, $i=1, \cdots, 7$.

One can use a packaged program to solve (8.9) to any desired degree of accuracy for $z$. Thereafter one gets, in turn, $w, v_{2}, v_{1}$ (from (8.8)), and $y_{1}, y_{2}$ (from $\left.(8.5)^{\prime}\right)$. One admits only common solutions which lie in $\pi\left(V^{-}\right)$.

9. $\Gamma(\varphi)$ and its automorphisms.

\section{1. $\Gamma(\varphi)$ and some of its elements.}

Let $I^{\prime}(\varphi)$ denote the group generated by $\boldsymbol{C}$-reflections corresponding to the diagram

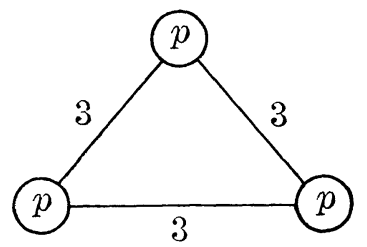

${ }^{2}$ The solution presented here is an improvement of the original solution. I am indebted to Dr. Sidnie Feit for this solution. 
where the corresponding hermitian form $H_{\varphi}$ is given by

$$
\left\langle e_{1}, e_{2}\right\rangle=\left\langle e_{2}, e_{3}\right\rangle=\left\langle e_{3}, e_{1}\right\rangle=-\frac{1}{2 \sin \frac{\pi}{p}} \varphi,
$$

$\varphi \in C,|\varphi|=1$. Select $p$ so that $1 / p+1 / p+2 / 3>1$, i.e., $p<6$. Set $\alpha=1 /(2 \sin \pi / p)$,

$$
\begin{aligned}
V & =\boldsymbol{C} e_{1}+\boldsymbol{C} e_{2}+\boldsymbol{C} e_{3} \\
\Delta & =\operatorname{det}\left(\left\langle e_{i}, e_{j}\right\rangle\right) \quad(i, j=1,2,3) \\
& =\operatorname{det}\left(\begin{array}{ccc}
1 & -\alpha \varphi & -\alpha \bar{\varphi} \\
-\alpha \varphi & 1 & -\alpha \varphi \\
-\alpha \varphi & -\alpha \bar{\varphi} & 1
\end{array}\right) \\
& =1-3 \alpha^{2}-\alpha^{3}\left(\varphi^{3}+\bar{\varphi}^{3}\right) .
\end{aligned}
$$

Set $\varphi=e^{i \theta}$. Then $\Delta=1-3 \alpha^{2}-2 \alpha^{3} \cos 3 \theta$. Recalling that $\cos 3 \theta=$ $4 \cos ^{3} \theta-3 \cos \theta$, we find that for $\cos \theta=1 / 2 \alpha$,

$$
\begin{aligned}
& \Delta=1-3 \alpha^{2}-2 \alpha^{3}\left(4 \cdot \frac{1}{8 \alpha^{3}}-3 \cdot \frac{1}{2 \alpha}\right)=0, \\
& \Delta<0 \text { if and only if } \cos \theta>\frac{1}{2 \alpha} ;
\end{aligned}
$$

that is, $\cos \theta>\sin \pi / p$ or $|\theta|<\pi / 2-\pi / p$. Thus the hermitian form $H$ has signature (two + , one -) only for $p>2$. Therefore for $p=3,4,5$ and

$$
\arg \left(\varphi^{3}\right)<3\left(\frac{\pi}{2}-\frac{\pi}{p}\right)
$$

the group $\Gamma(\varphi)$, which preserves the hermitian form $H_{\varphi}$ is embedded in $U(2,1)$. Hereafter we impose these conditions on $p$ and $\varphi$. We write $V(\varphi)$ for the vector space $V$ with $H_{\varphi}$ as inner product; when there is no ambiguity, we write $V$ for $V(\varphi)$.

Set $\eta=\exp (\pi \sqrt{-1} / p), \quad R_{i}(x)=x+\left(\eta^{2}-1\right)\left\langle x, e_{i}\right\rangle e_{i} \quad(i=1,2,3)$. The $C$-reflection $R_{i}$ depends on $\varphi$ and we sometimes write it as $R_{i}(\varphi)$. Since $\left(\eta^{2}-1\right) \alpha=\left(\eta^{2}-1\right) i /\left(\eta-\eta^{-1}\right)=\eta i$ we find

$$
\begin{aligned}
& R_{1}=\left(\begin{array}{ccc}
\eta^{2} & -\eta i \bar{\varphi} & -\eta i \varphi \\
0 & 1 & 0 \\
0 & 0 & 1
\end{array}\right) R_{2}=\left(\begin{array}{ccc}
1 & 0 & 0 \\
-\eta i \varphi & \eta^{2} & -\eta i \bar{\varphi} \\
0 & 0 & 1
\end{array}\right) R_{3}=\left(\begin{array}{ccc}
1 & 0 & 0 \\
0 & 1 & 0 \\
-\eta i \bar{\varphi} & -\eta i \varphi & \eta^{2}
\end{array}\right) \\
& R_{1} R_{2}=\left(\begin{array}{ccc}
0 & -\eta^{3} i \bar{\varphi} & -\eta^{2} \bar{\varphi}^{2}-\eta i \varphi \\
-\eta i \varphi & \eta^{2} & -\eta i \bar{\varphi} \\
0 & 0 & 1
\end{array}\right)
\end{aligned}
$$




$$
\begin{aligned}
& R_{1} R_{2} R_{3}=\left(\begin{array}{ccc}
\eta^{3} i \bar{\varphi}^{3}-\eta^{2} & -\eta^{2} \varphi^{2} & -\eta^{4} \bar{\varphi}^{2}-\eta^{3} i \varphi \\
-\eta i \varphi-\eta^{2} \bar{\varphi}^{2} & 0 & -\eta^{3} i \bar{\varphi} \\
-\eta i \bar{\varphi} & -\eta i \varphi & \eta^{2}
\end{array}\right) \\
& \left(R_{1} R_{2} R_{3}\right)^{2}=\left(\begin{array}{ccc}
-\eta^{6} \bar{\varphi}^{6}-\eta^{5} i \bar{\varphi}^{3}+\eta^{4}+\eta^{3} \varphi^{3} i & 0 & -\varphi \eta^{3} i\left(\eta^{4} \bar{\varphi}^{6}-\eta^{2}+\eta^{3} i \varphi^{3}\right) \\
-\eta^{5} i \bar{\varphi}^{5}+\eta^{4} \bar{\varphi}^{2}+\eta^{3} i \varphi & \eta^{3} i \varphi^{3} & \varphi^{2} \eta^{4}\left(-1+\eta^{2} \bar{\varphi}^{6}+\eta i \bar{\varphi}^{3}\right) \\
\eta^{4} \bar{\varphi}^{4}-\eta^{2} \varphi^{2}+\eta^{3} i \bar{\varphi} & 0 & -\eta^{4}-\eta^{5} i \bar{\varphi}^{3}
\end{array}\right) \\
& \left(R_{3} R_{2} R_{1}\right)^{2}=\left(\begin{array}{ccc}
-\eta^{4}-\eta^{5} i \varphi^{3} & 0 & \eta^{4} \varphi^{4}-\eta^{2} \bar{\varphi}^{2}-\eta^{3} i \varphi \\
\bar{\varphi}^{2} \eta^{4}\left(-1+\eta^{2} \varphi^{6}+\eta i \varphi^{3}\right) & \eta^{3} i \bar{\varphi}^{3} & -\eta^{3} i \varphi^{5}+\eta^{4} \varphi^{2}+\eta^{3} i \bar{\varphi} \\
-\bar{\varphi} \eta^{3} i\left(\eta^{4} \varphi^{6}-\eta^{2}+\eta^{3} i \varphi^{3}\right) & 0 & -\eta^{6} \phi^{6}-\eta^{5} i \varphi^{3}+\eta^{4}+\eta^{3} \bar{\varphi}^{3} i
\end{array}\right) \\
& R_{2} R_{1} R_{2} R_{3}=\left(\begin{array}{ccc}
\eta^{3} i \bar{\varphi}^{3}-\eta^{2} & -\eta^{2} \varphi^{2} & -\eta^{3} \varphi\left(\eta \bar{\varphi}^{3}+i\right) \\
-\eta^{2} \varphi^{2} & i \eta^{2}\left(\eta \varphi^{3}+i\right) & -\eta^{3} \bar{\varphi}\left(\eta \varphi^{3}+i\right) \\
-\eta i \bar{\varphi} & -\eta i \varphi & \eta^{2}
\end{array}\right) .
\end{aligned}
$$

The characteristic polynomial of $R_{1} R_{2} R_{3}$ is:

$$
-\lambda^{3}+\lambda^{2}\left[\eta^{3} i \bar{\varphi}^{3}\right]-\lambda\left[\eta^{4}-\left(\eta^{3} i \varphi^{3}+\eta^{4}\right)+\left(\eta^{5} i \bar{\varphi}^{3}-\eta^{4}-\left(\eta^{5} i \bar{\varphi}^{3}-\eta^{4}\right)\right]+\eta^{6}\right.
$$

and its roots satisfy

$$
\lambda^{3}-\lambda^{2}\left[\eta^{3} i \bar{\varphi}^{3}\right]-\lambda\left[\eta^{3} i \varphi^{3}\right]-\eta^{6}=0
$$

which factors

$$
\left(\lambda^{2}-\eta^{3} i \varphi^{3}\right)\left(\lambda-\eta^{3} i \bar{\varphi}^{3}\right)=0
$$

yielding as eigenvalues $\lambda=\eta^{3} i \bar{\varphi}^{3}$ and the roots of $\lambda^{2}=\eta^{3} i \varphi^{3}$. Similarly, the eigenvalues of $R_{3} R_{2} R_{1}$ satisfy

$$
\lambda^{3}-\lambda^{2}\left[\eta i \varphi^{3}\right]-\lambda\left[\eta i \bar{\varphi}^{3}\right]-\eta^{6}=0 ;
$$

this yields $\lambda=\eta^{3} i \varphi^{3}$ and the roots of $\lambda^{2}=\eta^{3} i \varphi^{-3}$.

The eigenvalues of $\left(R_{1} R_{2} R_{3}\right)^{2}$ are therefore

$$
-\eta^{6} \bar{\Phi}^{6}, \eta^{3} i \varphi^{3}, \eta^{3} i \varphi^{3}
$$

and the eigenvalues of $\left(R_{3} R_{2} R_{1}\right)^{2}$ are

$$
-\eta^{6} \varphi^{6}, \eta^{3} i \bar{\varphi}^{3}, \eta^{3} i \bar{\varphi}^{3}
$$

We next compute the image of $\left(R_{1} R_{2} R_{3}\right)^{2}-\eta^{3} i \varphi^{3} \times 1_{3}$ where $1_{s}$ denotes the identity $3 \times 3$ matrix.

$$
\begin{aligned}
& \left(R_{1} R_{2} R_{3}\right)^{2}-\eta^{3} i \varphi^{3} \cdot 1_{3} \\
& \quad=\left(-\eta^{2} i \bar{\varphi}^{3}+i \varphi^{3}+\eta\right) \bar{\varphi}^{2} \eta^{3}\left(\begin{array}{ccc}
-\bar{\varphi} \eta i & 0 & -\bar{\varphi} \eta i \cdot \varphi \eta i \\
1 & 0 & 1 \cdot \varphi \eta i \\
\varphi \bar{\eta} i & 0 & \varphi \bar{\eta} i \cdot \varphi \eta i
\end{array}\right) .
\end{aligned}
$$


Set

$$
\begin{aligned}
& v_{123}=\left(\begin{array}{c}
-\bar{\varphi} \eta i \\
1 \\
\varphi \bar{\eta} i
\end{array}\right) \quad v_{231}=\left(\begin{array}{c}
\varphi \bar{\eta} i \\
-\bar{\varphi} \eta i \\
1
\end{array}\right) \quad v_{312}=\left(\begin{array}{c}
1 \\
\varphi \bar{\eta} i \\
-\bar{\varphi} \bar{\eta} i
\end{array}\right) \\
& v_{321}=\left(\begin{array}{c}
\bar{\varphi} \bar{\eta} i \\
1 \\
-\varphi \eta i
\end{array}\right) \quad v_{132}=\left(\begin{array}{c}
-\varphi \eta i \\
\bar{\varphi} \bar{\eta} i \\
1
\end{array}\right) \quad v_{213}=\left(\begin{array}{c}
1 \\
-\varphi \eta i \\
\bar{\varphi} \bar{\eta} i
\end{array}\right) .
\end{aligned}
$$

$v_{i j k}$ is the normal in $V(\varphi)$ to the multiplicity-two eigenspace of $\left(R_{i} R_{j} R_{k}\right)^{2}$ where $i, j, k$ denotes a permutation of $1,2,3$.

$$
\begin{aligned}
\left\langle v_{123},\right. & \left.v_{123}\right\rangle=\left\langle-\bar{\varphi} \eta i e_{1}+e_{2}+\varphi \bar{\eta} i e_{3},-\bar{\varphi} \eta i e_{1}+e_{2}+\phi \bar{\eta} i e_{3}\right\rangle \\
& =3-\frac{i}{\eta-\eta}[-\bar{\varphi} \eta i(\varphi-\bar{\varphi} \eta i \bar{\varphi})+(\varphi \bar{\eta} i \bar{\varphi}-\bar{\varphi} \eta i \varphi)+\varphi \bar{\eta} i(\varphi \bar{\eta} i \varphi+\bar{\varphi})] \\
& =3-\frac{i}{\eta-\bar{\eta}}\left[-\eta i-\bar{\varphi}^{3} \eta^{2}+\bar{\eta} i-\eta i-\varphi^{3} \bar{\eta}^{2}+\bar{\eta} i\right] \\
& =3-\frac{i}{\eta-\bar{\eta}}\left[-i(\eta-\bar{\eta}) \cdot 2-\varphi^{3} \bar{\eta}^{2}-\bar{\eta}^{3} \eta^{2}\right] \\
& =1+\frac{i}{\eta-\bar{\eta}}\left(\phi^{3} \bar{\eta}^{2}+\bar{\varphi}^{3} \eta^{2}\right)
\end{aligned}
$$

$$
\begin{gathered}
=1+\frac{\cos \left(\frac{2 \pi}{p}-3 \theta\right)}{\sin \frac{\pi}{p}}, 3 \theta=\arg \varphi^{3} . \\
\left\langle v_{321}, v_{321}\right\rangle=1+\frac{i}{\eta-\bar{\eta}}\left(\bar{\varphi}^{3} \bar{\eta}^{2}+\phi^{3} \eta^{2}\right) \\
=1+\frac{\cos \left(\frac{2 \pi}{p}+3 \theta\right)}{\sin \frac{\pi}{p}} .
\end{gathered}
$$

We have

$$
\left\langle v_{123}, v_{123}\right\rangle>0 \text { if and only if }
$$

$$
-\left(\frac{\pi}{2}-\frac{\pi}{p}\right)<\arg \varphi^{3}<3\left(\frac{\pi}{2}-\frac{\pi}{p}\right)
$$

and

$$
\left\langle v_{321}, v_{321}\right\rangle>0 \text { if and only if }
$$

$$
-3\left(\frac{\pi}{2}-\frac{\pi}{p}\right)<\arg \varphi^{3}<\frac{\pi}{2}-\frac{\pi}{p} .
$$


Similar computation shows that the characteristic polynomial of $R_{2} R_{1} R_{2} R_{3}$ is

$$
-\lambda^{3}+\lambda^{2}\left[\eta^{3} i \bar{\varphi}^{3}+\eta^{3} i \varphi^{3}-\eta^{2}\right]-\lambda\left[-\eta^{6}-\eta^{5} i-\varphi^{5} i \bar{\varphi}\right]+\eta^{8}=0
$$

so that its eigenvalues are

$$
\eta^{3} i \varphi^{3}, \eta^{3} i \bar{\varphi}^{3},-\eta^{2}
$$

9.2. Automorphisms of $\Gamma(\varphi)$.

Let $J$ and $J^{\prime}$ denote the linear maps of $V$ onto itself given by:

$$
\begin{aligned}
& J: x_{1} e_{1}+x_{2} e_{2}+x_{3} e_{3} \longrightarrow x_{1} e_{2}+x_{2} e_{3}+x_{3} e_{1} \\
& J^{\prime}: x_{1} e_{1}+x_{2} x_{2}+x_{3} e_{3} \longrightarrow x_{1} e_{3}+x_{2} e_{2}+x_{3} e_{1} .
\end{aligned}
$$

Then $J$ is an isometry of $V(\varphi)$ to $V(\varphi)$, and $J^{\prime}$ is an isometry of $V(\varphi)$ to $V(\bar{\varphi})$ with $J^{\prime}$ of order two. Since $H_{\varphi}\left(e_{1}, e_{2}\right)=-\alpha \varphi=$ $H_{\bar{\varphi}}\left(e_{2}, e_{1}\right)=H_{\bar{\varphi}}\left(e_{3}, e_{2}\right)$.

Define

$$
\kappa: \sum_{i=1}^{3} x_{i} e_{i} \longrightarrow \sum_{i=1}^{3} \bar{x}_{i} e_{i} .
$$

Then $\kappa$ is a semi-linear map of $V(\varphi)$ to $V(\bar{\varphi})$ which is an isometry since,

$$
\begin{aligned}
H_{\bar{\varphi}}\left(\kappa\left(\sum_{i} x_{i} e_{i}\right), \kappa\left(\sum_{i} x_{i} e_{i}\right)\right) & =H_{\bar{\varphi}}\left(\sum \bar{x}_{i} e_{i}, \sum \bar{x}_{i} e_{i}\right) \\
& =\sum_{i, j} \bar{x}_{i} x_{j} H_{\bar{\varphi}}\left(e_{i}, e_{j}\right) \\
& =\sum_{i, j} x_{i} \bar{x}_{j} H_{\varphi}\left(e_{i}, e_{j}\right) \\
& =H_{\varphi}\left(\sum x_{i} e_{i}, \sum x_{i} e_{i}\right) .
\end{aligned}
$$

Regarding the index as an integer modulo 3 , we have

$$
\begin{aligned}
& J\left(R_{i}(\varphi)\right)=R_{i+1}(\varphi) \\
& J^{\prime}\left(R_{i}(\varphi)\right)=R_{1-i}(\bar{\varphi}) \\
& \kappa\left(R_{i}(\varphi)\right)=\overline{R_{i}(\varphi)}=R_{i}(\bar{\varphi})^{-1} \quad i=1,2,3 .
\end{aligned}
$$

Set $a_{13}=J^{\prime} \kappa$. Then $a_{13}$ is a semi-linear isometry of $V(\varphi)$ to $V(\varphi)$ with

$$
a_{13}: R_{i}(\varphi) \longrightarrow R_{1-i}(\varphi)^{-1} \quad 1=1,2,3 .
$$

Set $a_{21}=J \alpha_{13} J^{-1}, a_{32}=J^{-1} a_{13} J$.

The group of isometries of $V(\varphi)$ generated by $a_{12}$ and $J$ is of order 6 -these are the isometries which permute $e_{1}, e_{2}, e_{3}$. (For $\varphi=1$, there is the additional isometry $\kappa$.) The subgroup of linear isometries is of order 3. All these isometries induce automorphisms 
of $\Gamma(\varphi)$.

10. The region $F(\varphi)$.

10.1. A fundamential domain for $\Gamma_{i j}$.

Set $p_{0}=e_{1}+e_{2}+e_{3}$. Then

$$
\left\langle p_{0}, e_{1}\right\rangle=\left\langle p_{0}, e_{2}\right\rangle=\left\langle p_{0}, e_{3}\right\rangle=1-\alpha(\varphi+\bar{\varphi})
$$

As in $\S 3$, we take as our model of hermitian hyperbolic space $C h^{2}$ the image in projective space $C P^{2}$ under the map $\pi: V-\{0\} \rightarrow C P^{2}$ of the negative cone

$$
V^{-}=\left\{z \in V ;\langle z, z\rangle\langle 0\} \quad\left(=\text { the ball in } C^{2}\right)\right.
$$

with metric

$$
d\left(z_{1}, z_{2}\right)=\cosh ^{-1}\left(\frac{\left\langle z_{1}, z_{2}\right\rangle\left\langle z_{2}, z_{1}\right\rangle}{\left\langle z_{1}, z_{1}\right\rangle\left\langle z_{2}, z_{2}\right\rangle}\right)^{1 / 2}
$$

Since $\left\langle p_{0}, p_{0}\right\rangle=3(1-\alpha(\varphi+\bar{\varphi})),\left\langle p_{0}, p_{0}\right\rangle<0$ for $|\arg \varphi|<\pi / 2-\pi / p$.

We denote by $p_{0}$ also, the image of $p_{0}$ in $C h^{2}$. For any $\varphi \in \Gamma$, set

$$
\begin{aligned}
& \gamma^{+}=\left\{x \in C h^{2} ; d\left(x, p_{0}\right) \leqq d\left(\gamma x, p_{0}\right)\right\} \\
& \hat{\gamma}=\left\{x \in C h^{2} ; d\left(x, p_{0}\right)=d\left(\gamma x, p_{0}\right)\right\}
\end{aligned}
$$

Set $\Gamma_{i j}=\left\{\left\{R_{i}, R_{j}\right\}\right\}(i \neq j ; i, j \in(1,2,3))$ where $\{\{a, b\}\}$ denotes the group generated by $\{a, b\} . \quad \Gamma_{i j}$ is a finite group. Since $\Gamma_{i j}$ is irreducible on $C e_{1}+C e_{2}, \gamma p_{0} \neq p_{0}$ if $\gamma \in \Gamma_{i j}, \gamma \neq 1$. Set

$$
F_{i j}=\bigcap_{r \in i j} \gamma^{+} \text {. }
$$

Then $F_{i j}$ is a fundamental domain for $\Gamma_{i j}$. Set $e_{i}^{\downarrow}=\left\{z \in V ;\left\langle z, e_{i}\right\rangle=\right.$ $0\}$ and denote also by $e_{i}^{\frac{1}{i}}$ the subset $\pi\left(e_{i}^{+} \cap V^{-}\right)$of $C h^{2} i=1,2,3$. Then $e_{i}^{L}$ is the fixed point set of $R_{i}$ whether in $V$ or in $C h^{2}$.

Set $p_{i j}=\pi\left(e_{i}^{\perp} \cap e_{j}^{\perp}\right)$. The equation of $e_{i}^{\perp}$ is given by:

$$
\begin{aligned}
& e_{1}^{\perp}: 0=\left\langle x_{1} e_{1}+x_{2} e_{2}+x_{3} e_{3}, e_{1}\right\rangle=x_{1}-\alpha \bar{\varphi} x_{2}-\alpha \varphi x_{3} \\
& e_{2}^{\perp}: 0=\left\langle x_{1} e_{1}+x_{2} e_{2}+x_{3} e_{3}, e_{2}\right\rangle=-\alpha \varphi x_{1}+x_{2}-\alpha \bar{\varphi} x_{3} \\
& e_{3}^{\perp}: 0=\left\langle x_{1} e_{1}+x_{2} e_{2}+x_{3} e_{3}, e_{3}\right\rangle=-\alpha \bar{\varphi} x_{1}-\alpha \varphi x_{2}+x_{3} \\
& p_{12}: x_{1}=x_{3}\left|\begin{array}{cc}
\alpha \varphi & -\alpha \bar{\varphi} \\
\frac{\alpha \bar{\varphi}}{1} & 1 \\
1 & -\alpha \bar{\varphi} \\
-\alpha \varphi & 1
\end{array}\right|=x_{3} \frac{\alpha \varphi+\alpha^{2} \bar{\varphi}^{2}}{1-\alpha^{2}}, x_{2}=x_{3} \frac{\alpha \bar{\varphi}+\alpha^{2} \bar{\varphi}^{2}}{1-\alpha^{2}} .
\end{aligned}
$$


Thus $p_{12}=\pi\left(\varphi \alpha\left(1+\alpha \bar{\varphi}^{3}\right), \bar{\varphi} \alpha\left(1+\alpha \varphi^{3}\right), 1-\alpha^{2}\right)$. Set $\xi=\varphi \alpha\left(1+\alpha \bar{\varphi}^{3}\right)$. Then $\left\langle p_{12}, p_{12}\right\rangle=\left(1-\alpha^{2}\right) \Delta<0, p_{12}=\pi\left(\xi, \bar{\xi}, 1-\alpha^{2}\right), p_{23}=\pi\left(1-\alpha^{2}, \xi, \bar{\xi}\right)$, $p_{31}=\pi\left(\bar{\xi}, 1-\alpha^{2}, \xi\right)$. We also write $p_{12}=\left(\xi, \bar{\xi}, 1-\alpha^{2}\right)$ and similarly for $p_{23}, p_{31}$.

For any $\gamma \in I_{12}$, the equation of $\hat{\gamma}$ is given by

$$
\left|\left\langle x, p_{0}\right\rangle\right|=\left|\left\langle\gamma x, p_{0}\right\rangle\right|
$$

since $\langle x, x\rangle=\langle\gamma x, \gamma x\rangle$. Since $R_{1} p_{12}=p_{12}=R_{2} p_{12}$, we have $\gamma p_{12}=p_{12}$ for all $\gamma \in \Gamma_{12}$ and thus $p_{12} \in \hat{\gamma}$ for all $\gamma \in \Gamma_{12} . \quad F_{12}$ is a curvilinear solid angle with apex at $p_{12}$. We must know the 3 -faces of $F_{12}$. In principle, the 3 -faces of $F_{12}$ in some neighborhood of the apex $p_{12}$ can be computed by hand thanks to Lemma 3.2.9. For by that lemma, the tangent planes to each $\hat{\gamma}$ at $p_{12}$ are distinct for all $\gamma \in$ $F_{12}$. Hence the fundamental domain $\dot{F}_{12}$ for $I_{12}$ acting on the tangent space to $C h^{2}$ at $p_{12}$ determines the faces of $F_{12}$ near $p_{12}$. The computation of the faces of $\dot{F}_{12}$ involves the solution of a system of $24(p / 6-p)^{2}$ linear inequalities $(p=3,4,5)$ and in principal this can be done by hand.

For any $r>0$, set

$$
S_{r}=\left\{x \in C h^{2} ; d\left(x, p_{12}\right)=r\right\} .
$$

Then $S_{r}$ is stable under $\Gamma_{12}$. A fundamental domain $F_{12}^{*}$ for $I_{12}$ on $S_{r}$ can be calculated (for any fixed $\varphi$ ) via computer.

In Figure 1, we exhibit the domain $F_{12}^{*}$ with $r=d\left(p_{12}, p_{0}\right)$. The faces of $F_{1.2}^{*}$ correspond to the 10 elements

$$
R_{1}^{ \pm 1}, R_{2}^{ \pm 1},\left(R_{1} R_{2}\right)^{ \pm 1},\left(R_{2} R_{1}\right)^{ \pm 1},\left(R_{1} R_{2} R_{1}\right)^{ \pm 1}
$$

of the finite group $\Gamma_{12}$. In Figures 2, 3, and 4, we show the 3-faces of $F_{12}$ which lie on the surfaces $\hat{R}_{1}, \widehat{R_{1} R_{2}}$, and $R_{1} \hat{R}_{2} R_{1}$. The definition of the vertices $s_{2 j}, t_{i k}$ are given in $\S 11$.

From the remark following Theorem 6.3.2, we know that $F_{12}$ satisfies condition

$$
\gamma^{-1}\left(\hat{\gamma}^{-1} \cap F_{12}\right)=\hat{\gamma} \cap F_{12}
$$

for all $\gamma \in\left\{R_{1}^{ \pm 1}, R_{2}^{ \pm 1},\left(R_{1} R_{2}\right)^{ \pm 1},\left(R_{2} R_{1}\right)^{ \pm 1},\left(R_{1} R_{2} R_{1}\right)^{ \pm 1}\right\}$ and also condition (CD2). This last condition yields for each two faces of $F_{12}$ an identity corresponding to the circuit $F_{1} \gamma_{1} F, \gamma_{2} \gamma_{2} F, \cdots$ where

$$
e=\hat{\gamma}_{0} \cap \hat{\gamma}_{1}^{-1} \cap F_{12}, \gamma_{1}^{-1}(e)=\hat{\gamma}_{1} \cap \hat{\gamma}_{2}^{-1} \cap F_{12}, \gamma_{2}^{-1} \gamma_{1}^{-1} e=\hat{\gamma}_{2} \cap \hat{\gamma}_{33}^{-1} \cap F_{12}, \cdots,
$$

and $\gamma_{1} \gamma_{2} \cdots \gamma_{n} F_{12} \cap F_{12}$ has a nonempty interior; namely

$$
\gamma_{1} \gamma_{2} \cdots \gamma_{n}=1
$$




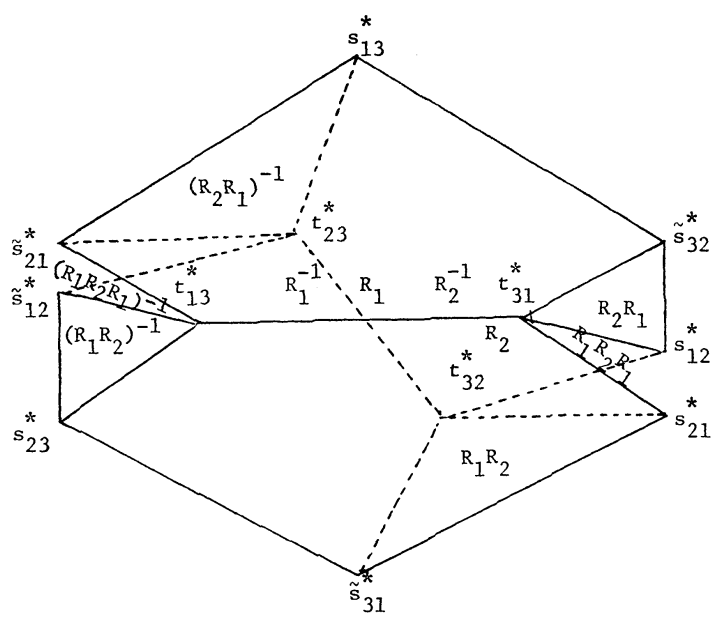

Figure 10.1. The intersection $F_{12}^{*}$ of $F_{12}$ with a 3 -sphere $S_{r}$ centered at $p_{12}$ of radius $r=d\left(p_{12}, p_{0}\right)$.

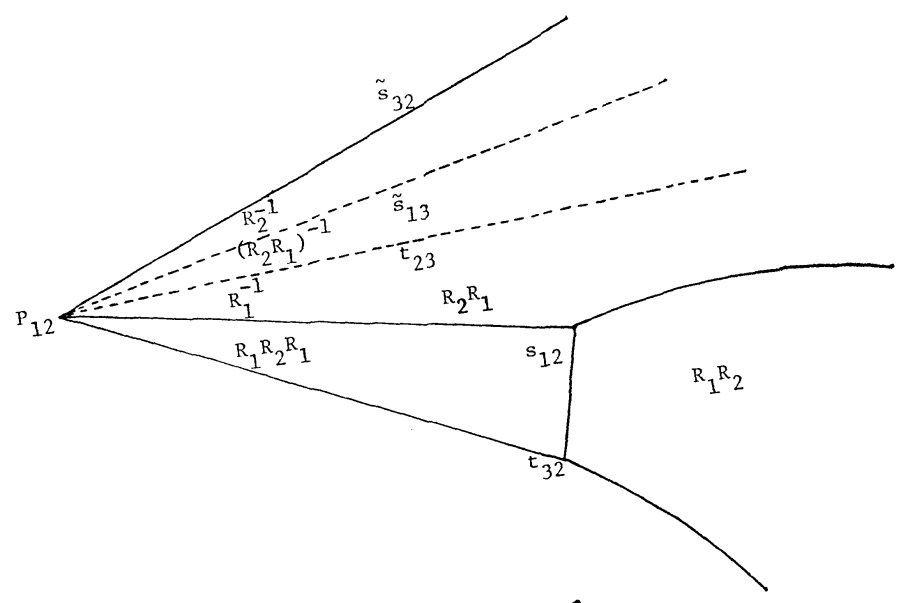

FIGURE 10.2. $\quad F_{12} \cap \hat{R}_{1}$

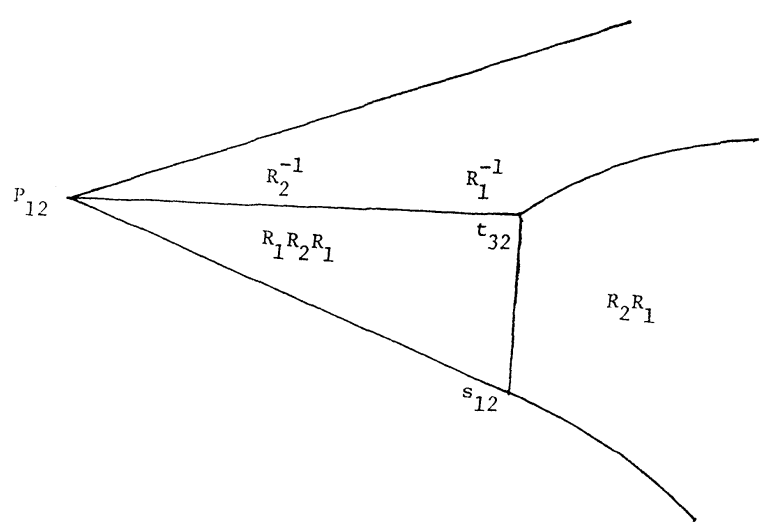

FIGURE 10.3. $\quad F_{12} \cap\left(\widehat{R_{1} R_{2}}\right)$ 
From Figure 1, one can read off the elements of $E_{2}\left(F_{12}\right)$ which contain $p_{12}$; they correspond to the twenty edges of $F_{12} \cap S_{r}$. For any $\gamma$ such that $\hat{\gamma} \cap F_{12} \in E_{1}\left(F_{12}\right)$, set

$$
{ }^{*} \gamma=\hat{\gamma} \cap F_{12} .
$$

Then along with the knowledge of $E_{1}\left(F_{12}\right)$ one can determine the circuits around each $e \in E_{2}\left(F_{12}\right)$ and the corresponding identity. For ${ }^{*} R_{i} \cap{ }^{*} R_{i}^{-1}$, the circuit yields the identity $R_{i}^{p}=1 \quad(i=1,2)$. The remaining eighteen 2 -faces of $F_{12}$ occur in circuits of three terms each

$$
\begin{aligned}
& { }^{*} R_{1} \cap{ }^{*}\left(R_{2} R_{1}\right) \\
& \left(R_{2} R_{1}\right)^{-1} \nearrow \quad \backslash R_{1} \quad\left(R_{2} R_{1}\right)^{-1} R_{2} R_{1}=1 \\
& *\left(R_{2} R_{1}\right)^{-1} \cap * R_{2}^{1-1} \stackrel{R_{2}}{\longleftarrow} R_{2} \cap * R_{1}^{-1} \\
& { }^{*} R_{2} \cap *\left(R_{1} R_{2}\right) \\
& \left(R_{1} R_{2}\right)^{-1} \nearrow \quad \backslash R_{2} \quad\left(R_{1} R_{2}\right)^{-1} R_{1} R_{2}=1 \\
& *\left(R_{1} R_{2}\right)^{-1} \cap * R_{1}^{-1} \stackrel{R_{1}}{\longleftarrow}{ }^{*} R_{1} \cap R_{2}^{*-1} \\
& { }^{*} R_{1} \cap *\left(R_{1} R_{2} R_{1}\right) \\
& \left(R_{1} R_{2} R_{1}\right)^{-1} \nearrow \\
& \backslash R_{1} \\
& *\left(R_{1} R_{2} R_{1}\right)^{-1} \cap *\left(R_{1} R_{2}\right)^{-1} \stackrel{R_{1} R_{2}}{\longleftarrow} * R_{1} R_{2} \cap * R_{1}^{-1} \\
& { }^{*} R_{2} \cap *\left(R_{1} R_{2} R_{1}\right) \\
& \left(R_{1} R_{2} R_{1}\right)^{-1} \nearrow \quad \backslash R_{2} \quad\left(R_{1} R_{2} R_{1}\right)^{-1} R_{2} R_{1} R_{2}=1 \\
& *\left(R_{1} R_{2} R_{1}\right)^{-1} \cap *\left(R_{2} R_{1}\right)^{-1} \stackrel{R_{2} R_{1}}{\longleftarrow} *\left(R_{2} R_{1}\right)^{-1} \cap * R_{2}^{-1} \\
& *\left(R_{1} R_{2}\right) \cap *\left(R_{1} R_{2} R_{1}\right) \\
& \left(R_{1} R_{2} R_{1}\right)^{-1} \nearrow \backslash R_{1} R_{2} \quad\left(R_{1} R_{2} R_{1}\right)^{-} R_{2} R_{1} R_{2}=1 \\
& *\left(R_{1} R_{2} R_{1}\right)^{-1} \cap * R_{2}^{-1} \stackrel{R_{2}}{\longleftarrow} * R_{2} \cap *\left(R_{1} R_{2}\right)^{-1} \\
& { }^{*}\left(R_{2} R_{1}\right) \cap{ }^{*}\left(R_{1} R_{2} R_{1}\right) \\
& \left(R_{1} R_{2} R_{1}\right)^{-1} \nearrow \backslash R_{2} R_{1} \quad\left(R_{1} R_{2} R_{1}\right)^{-1} R_{1} R_{2} R_{1}=1 . \\
& *\left(R_{1} R_{2} R_{1}\right)^{-1} \cap * R_{1}^{-1} \stackrel{R_{1}}{\longleftarrow} R_{1} \cap *\left(R_{2} R_{1}\right)^{-1}
\end{aligned}
$$

REMARK. In $\S 13$, we shall show the eighteen mappings of (10.1.2) can be verified easily. Thus these circuits yield the presentation

$$
R_{1}^{p}=1=R_{2}^{p}, \quad R_{1} R_{2} R_{1}=R_{2} R_{1} R_{2}
$$

of the finite group $\Gamma_{12}$. 
The isometry $a_{12}$ corresponding to the automorphism $R_{1} \rightarrow R_{2}^{-1}$, $R_{2} \rightarrow R_{1}^{-1}$ of $\Gamma_{12}$ permutes the elements of $E_{1}\left(F_{12}\right)$ sending $*\left(R_{1} R_{2}\right)$ to ${ }^{*}\left(R_{2}^{-1} R_{1}^{-1}\right)={ }^{*}\left(R_{1} R_{2}\right)^{-1}{ }^{*}\left(R_{2} R_{1}\right)$ to ${ }^{*}\left(R_{2} R_{1}\right)^{-1}$, and ${ }^{*}\left(R_{1} R_{2} R_{1}\right)$ to ${ }^{*}\left(R_{1} R_{2} R_{1}\right)^{-1}$.

The isometry $a_{12}$ sends $E_{2}\left(F_{12}\right)$ to $E_{2}\left(F_{12}\right)$ and thus we get two additional isometries

$$
\begin{aligned}
& { }^{*} R_{1} \cap *\left(R_{1} R_{2} R_{1}\right) \cong{ }^{*}\left(R_{1} R_{2} R_{1}\right)^{-1} \cap *\left(R_{1} R_{2}\right)^{-1} \cong{ }^{*}\left(R_{1} R_{2}\right) \cap *\left(R_{1} R_{2} R_{1}\right) \\
& { }^{*} R_{2} \cap *\left(R_{1} R_{2} R_{1}\right) \cong{ }^{*}\left(R_{1} R_{2} R_{1}\right)^{-1} \cap *\left(R_{2} R_{1}\right)^{-1} \cong *\left(R_{2} R_{1}\right) \cap *\left(R_{1} R_{2} R_{2}\right) .
\end{aligned}
$$

10.2. The domain $F(\varphi),|\arg \varphi|<\pi / 2-\pi / p$.

We continue the preceding notation. Set

$$
F(\varphi)=F_{12} \cap F_{23} \cap F_{31} .
$$

When there is no ambiguity, we write $F$ for $F(\varphi)$. In $\S 12$, we shall determine certain vertices of $F$. For the present we note that for all $\varphi$ with $|\arg \varphi|<\pi / 2-\pi / p, F$ is stable under the isometries $J$ and $\left\{a_{i j} ;(i \neq j, i, j=1,2,3)\right\}$ and that $J^{\prime}$ and $K^{\prime}$ are isometries of $F(\varphi)$ to $F(\bar{\varphi})$. We denote by Isom $F$ the group of six isometries of $V(\varphi)$ generated by $J$ and $a_{12}$.

It will turn out that the combinatorial scheme of $F$ and its faces remains unchanged for $\left|\arg \left(\varphi^{3}\right)\right|<\pi / 2-\pi / p$, is unbounded for $\arg \left(\varphi^{3}\right)=\pi / 2-\pi / p$, and becomes bounded, and combinatorially constant for $1 / 3(\pi / 2-\pi / p)<|\arg \varphi|<\pi / 2-\pi / 2$; but it is combinatorially different than the case $\left|\left(\arg \left(\Phi^{3}\right)\right)\right|<\pi / 2-\pi / p$.

\section{2-faces of $F(\varphi)$ not containing an apex.}

For any permutation $(i, j, k)$ of $(1,2,3)$, set

$$
I_{i j k}=\left(\widehat{R_{i} R_{j}}\right)^{-1} \cap \widehat{R_{j} R_{k}}
$$

and using indices modulo 3

$$
\begin{aligned}
& I_{j}=I_{j-1, j, j+1} \\
& I_{j}^{\prime}=I_{j+1, j, j-1} .
\end{aligned}
$$

LEMMA 11.1. $I_{i j k}$ is the common slice of $\left(\widehat{R_{i} R_{j}}\right)^{-1}$ and $\widehat{R_{j} R_{k}}$.

Proof. Let $J$ and $J^{\prime}$ denote the isometries defined in $\S 9.2$, Then clearly

$$
J I_{2}=I_{3} J^{-1} I_{2}=I_{1} \text {. }
$$

Moreover, $J^{\prime} R_{i}(\varphi)=R_{1-i}(\bar{\varphi})$ so that

$$
J^{\prime} I_{123}(\varphi)=I_{321}(\bar{\varphi}) .
$$


Consequently, it suffices to prove that $I_{2}$ is a common slice of $\left(\widehat{R_{1}} R_{2}\right)^{-1}$ and $\widehat{R_{2} R_{3}}$. We have

$$
\begin{aligned}
\left(R_{1} R_{2}\right)^{-1}=R_{2}^{-1} R_{1}^{-1} & =\left(\begin{array}{ccc}
1 & 0 & 0 \\
\bar{\eta} i \varphi & \bar{\eta}^{2} & \bar{\eta} i \bar{\varphi} \\
0 & 0 & 1
\end{array}\right)\left(\begin{array}{ccc}
\bar{\eta}^{2} & \bar{\eta} i \bar{\varphi} & \bar{\eta} i \varphi \\
0 & 1 & 0 \\
0 & 0 & 1
\end{array}\right) \\
& =\left(\begin{array}{cccc}
\bar{\eta}^{2} & \bar{\eta} i \bar{\varphi} & \bar{\eta} i \varphi & \\
\bar{\eta}^{3} i \varphi & 0 & -\bar{\eta}^{2} \varphi^{2}+\bar{\eta} i \bar{\varphi} \\
0 & 0 & 1
\end{array}\right) \\
R_{2} R_{3} & =\left(\begin{array}{ccc}
1 & 0 & 0 \\
-\eta i \varphi & \eta^{2} & -\eta i \bar{\varphi} \\
0 & 0 & 1
\end{array}\right)\left(\begin{array}{ccc}
1 & 0 & 0 \\
0 & 1 & 0 \\
-\eta i \bar{\varphi} & -\eta i \varphi & \eta^{2}
\end{array}\right) \\
& =\left(\begin{array}{cccc}
1 & 0 & 0 \\
-\eta i \varphi-\eta^{2} \bar{\varphi}^{2} & 0 & -\eta^{3} i \bar{\varphi} \\
-\eta i \bar{\varphi} & -\eta i \varphi & \eta^{2}
\end{array}\right) .
\end{aligned}
$$

In the homogeneous coordinates dual to $e_{1}, e_{2}, e_{3}$, we get the equations

$$
\begin{aligned}
& \left(\widehat{R_{1} R_{2}}\right)^{-1}:\left|\left(\bar{\eta}^{2}+\bar{\eta}^{3} i \varphi\right) x_{1}+\bar{\eta} i \bar{\varphi} x_{2}+\left(1+\bar{\eta} i \varphi-\bar{\eta}^{2} \varphi^{2}+\bar{\eta} i \bar{\varphi}\right) x_{3}\right|=1 \\
& \widehat{R_{2} R_{3}}:\left|\left(1-\eta i \bar{\varphi}-\eta^{2} \bar{\varphi}^{2}-\eta i \varphi\right) x_{1}-\eta i \varphi x_{2}+\left(\eta^{2}-\eta^{3} i \bar{\varphi}\right) x_{3}\right|=1
\end{aligned}
$$

multiplying the equation of $\left(\widehat{R_{1} R_{2}}\right)^{-1}$ by $-\eta^{3} i \bar{\varphi}$ it takes the form

$$
\left(\widehat{R_{1} R_{2}}\right)^{-1}:\left|(1-\eta i \bar{\varphi}) x_{1}+\eta^{2} \bar{\varphi}^{2} x_{2}+\left(\eta^{2}-\eta^{3} i \bar{\varphi}+\eta i \varphi+\eta^{2} \bar{\varphi}^{2}\right) x_{3}\right|=1
$$

or

$$
\left|\left(1-\eta i \bar{\varphi}-\eta^{2} \bar{\varphi}^{2}\right) x_{1}+\left(\eta^{2}-\eta^{3} i \bar{\varphi}+\eta i \varphi\right) x_{3}+\eta^{2} \bar{\varphi}^{2}\left(x_{1}+x_{2}+x_{3}\right)\right|=1 .
$$

\section{Similarly}

$$
\widehat{R_{2} R_{3}}:\left|\left(1-\eta i \bar{\varphi}-\eta^{2} \bar{\varphi}^{2}\right) x_{1}+\left(\eta^{2}-\eta^{3} i \bar{\varphi}+\eta i \varphi\right) x_{3}-\eta i \varphi\left(x_{1}+x_{2}+x_{3}\right)\right|=1 .
$$

Therefore, set

$$
z=\frac{\left(1-\eta i \bar{\varphi}-\eta^{2} \bar{\varphi}^{2}\right) x_{1}+\left(\eta^{2}-\eta^{3} i \bar{\varphi}+\eta i \varphi\right) x_{3}}{x_{1}+x_{2}+x_{3}}
$$

to obtain as equations

$$
\begin{aligned}
& \left(\widehat{R_{1} R_{2}}\right)^{-1}:\left|z+\eta^{2} \bar{\varphi}^{2}\right|=1 \\
& \widehat{R_{2} R_{3}}:|z-\eta i \varphi|=1 .
\end{aligned}
$$

These equations have 2 solutions (see figure): 


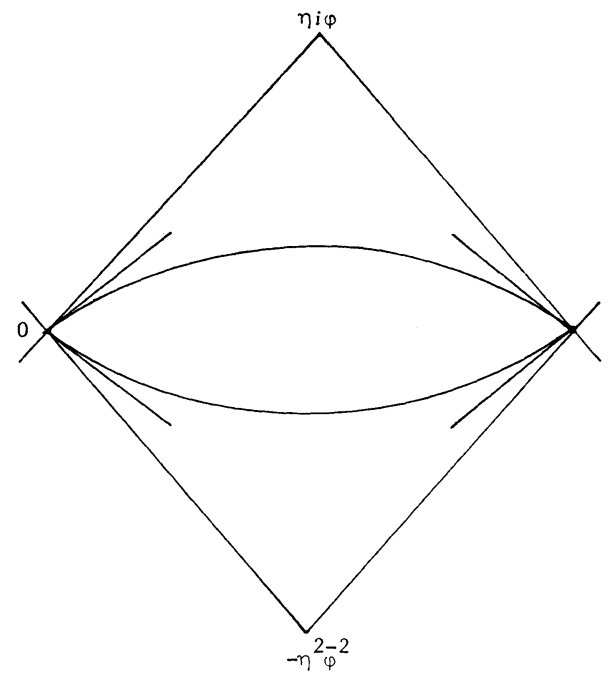

FIGURE 11.1

$$
z=0 \text { and } z=\eta i \varphi-\eta^{2} \bar{\varphi}^{2} \text {. }
$$

We note that $1-\eta i \bar{\varphi}-\eta^{2} \bar{\varphi}^{2}=-\bar{\eta} i \bar{\varphi}\left(\eta^{2}-\eta^{3} i \bar{\varphi}+\eta i \varphi\right)$ and thus

$$
z=\left(\eta^{2}-\eta^{3} i \bar{\varphi}+\eta i \varphi\right) \frac{\left(-\bar{\eta} i \bar{\varphi} x_{1}+x_{3}\right)}{x_{1}+x_{2}+x_{3}} .
$$

The above two solutions yield two possibilities for the preimage of $I_{2}$ and by Lemma 3.4.1, exactly one of two possibilities.

$$
\text { Case a: }-\bar{\eta} i \bar{\varphi} x_{1}+x_{3}=0
$$

$$
\text { Case b: } \quad-\bar{\eta} i \bar{\varphi} x_{1}+x_{3}=\frac{\eta i \varphi-\eta^{2} \bar{\varphi}^{2}}{\eta^{2}-\eta^{3} i \bar{\varphi}+\eta i \varphi}\left(x_{1}+x_{2}+x_{3}\right) \text {. }
$$

Case $\mathrm{b}$ is equivalent to

$$
\left(1-\eta i \bar{\varphi}-\eta^{2} \bar{\varphi}^{2}\right) x_{1}+\left(\eta^{2}-\eta^{3} i \bar{\varphi}+\eta i \varphi\right) x_{3}=\left(\eta i \varphi-\eta^{2} \bar{\varphi}^{2}\right)\left(x_{1}+x_{2}+x_{3}\right)
$$

or

$$
(1-\eta i \bar{\varphi}-\eta i \varphi) x_{i}-\left(\eta i \varphi-\eta^{2} \bar{\Phi}^{2}\right) x_{2}+\left(\eta^{2}-\eta^{3} i \bar{\varphi}+\eta^{2} \bar{\Phi}^{2}\right) x_{3}=0
$$

or

$$
\begin{array}{r}
(1-\eta i \varphi-\eta i \bar{\varphi}) x_{1}-\eta i \varphi\left(1+\eta i \bar{\varphi}^{3}\right) x_{2} \\
+\eta^{2}\left(1-\eta i \bar{\varphi}+\bar{\varphi}^{2}\right) x_{3}=0 .
\end{array}
$$

Applying $J$, the equation for the preimage of $I_{2}^{\prime}$ is exactly one of the two possibilities

Case a: $\quad-\bar{\eta} i \varphi x_{3}+x_{1}=0$ 
$(11.3 \mathrm{~b})^{\prime} \quad$ Case b: $\quad(1-\eta i \varphi) x_{3}-\eta i \bar{\varphi}\left(1-\eta i \varphi^{3}\right) x_{2}+\eta^{2}(1-\eta i \varphi) x_{1}=0$.

The next lemma determines the values of $\phi$ for which Cases a and $b$ apply.

LEMMA 11.2. Let $v_{i j k}$ be the point in $V$ defined in $\S 9.1$. $(i, j, k)$ being a permutation of $(1,2,3)$

(1) The equation of $\pi^{-1}\left(I_{2}\right)$ is given by $-\bar{\eta} i \bar{\varphi} x_{1}+x_{3}=0$ and $v_{123}$ is the normal in $V$ to $\pi^{-1}\left(I_{2}\right)$ for

$$
-\left(\frac{\pi}{2}-\frac{\pi}{p}\right)<\arg \left(\varphi^{3}\right)<3\left(\frac{\pi}{2}-\frac{\pi}{p}\right) ;
$$

and $\pi^{-1}\left(I_{2}\right)$ is given by $(11.3 \mathrm{~b})$ for $-3(\pi / 2-\pi / p)<\arg \varphi^{3} \leqq-(\pi / 2-$ $\pi / p)$.

(2) The equation of $\pi^{-1}\left(I_{2}^{\prime}\right)$ is given by $x_{1}-\bar{\eta} i \varphi x_{3}=0$ and $v_{321}$ is the normal in $V$ to $\pi^{-1}\left(I_{2}^{\prime}\right)$ for

$$
-3\left(\frac{\pi}{2}-\frac{\pi}{p}\right)<\arg \left(\varphi^{3}\right)<\frac{\pi}{2}-\frac{\pi}{p} ;
$$

and $\pi^{-1}\left(I_{2}\right)$ is given by $(11.3 b)^{\prime}$ for $\pi / 2-\pi / p \leqq \arg \varphi^{3}<3(\pi / 2-\pi / p)$.

Proof. Consider the orthogonal subspace $v_{123}^{\perp}$ to $v_{123}$ in $V$ with respect to the inner product $H$. Then $x_{1} e_{1}+x_{2} e_{2}+x_{3} e_{3}$ is in $v_{123}^{\perp}$ if and only if

$$
\begin{aligned}
& \left\langle x_{1} e_{1}+x_{2} e_{2}+x_{3} e_{3},-\eta i \bar{\varphi} e_{1}+e_{2}+\bar{\eta} i \varphi\right\rangle=0 \\
& x_{1}\left[\bar{\eta} i \varphi-\alpha\left(\varphi-\eta i \bar{\varphi}^{2}\right]+x_{2}\left[1-\alpha\left(\bar{\eta}^{i}-\eta i\right)\right]+x_{3}\left[-\eta i \bar{\varphi}-\alpha\left(\bar{\eta} i \varphi^{2}+\bar{\varphi}\right]=0 .\right.\right.
\end{aligned}
$$

Replace $\alpha$ by $i /(\eta-\bar{\eta})$; we find

$$
x_{1}\left[\varphi\left(-\bar{\eta}^{2} i-\eta \bar{\varphi}^{3}\right]+x_{3}\left[\bar{\varphi}\left(-\eta^{2} i+\bar{\eta} \varphi^{3}\right]=0 .\right.\right.
$$

Note that $\varphi\left(\bar{\eta}^{2} i+\bar{\eta}^{3}\right)=\bar{\eta} i \bar{\varphi} \cdot \bar{\varphi}\left(-\eta^{2} i+\bar{\eta} \varphi^{3}\right)$. Hence we get

$$
v_{123}^{1}:-\bar{\eta} i \bar{\varphi} x_{1}+x_{3}=0 \text {. }
$$

By (9.1.4), $\left\langle v_{123}, v_{123}\right\rangle>0$ for $-(\pi / 2-\pi / p)<\arg \varphi^{3}<3(\pi / 2-\pi / p)$. Hence for such $\varphi, v_{123}^{\perp} \cap V^{-}$is not empty and $\pi\left(v_{123}^{\perp} \cap V^{-}\right)$is a $C$-line in $C h^{2}$. Its equation is precisely that of $I_{2}$. Hence $I_{2}=\pi\left(v_{123}^{\perp} \cap V^{-}\right)$. For the remaining values of $\varphi, I_{2}$ is given by (11.3a). This proves (1). (2) follows from (1) by applying the isometry $J^{\prime}: V(\varphi) \rightarrow V(\bar{\varphi})$.

REMARK 1. In Case a, the preimage of $I_{2}$ in $V^{-}$has an equation independent of $x_{2}$. It follows at once that $I_{2}$ is stable under the $C$-reflection $R_{2}: x \rightarrow x+\left(\eta^{2}-1\right)\left\langle x, e_{2}\right\rangle e_{2}$, since $x_{1}\left(e_{2}\right)=x_{3}\left(e_{2}\right)=0$. Clearly $I_{2}$ is not pointwise fixed under $R_{2}$. Being stable under $R_{2}$, 
it must be orthogonal to $\pi\left(e_{2}^{1}\right)$, the fixed point set of $R_{2}$. From this it follows that the $C$-reflection in the $C$-line $I_{2}$ commutes with $R_{2}$. But by (9.1.3), $\left(R_{1} R_{2} R_{3}\right)^{2}$ multiplies each element of $v_{123}^{\perp}$ by the scalar $\eta^{3} i \varphi^{3}$. Thus $I_{2}$ is the fixed point set of $\left(R_{1} R_{2} R_{3}\right)^{2}$. Consequently $R_{2}$ commutes with $\left(R_{1} R_{2} R_{3}\right)^{2}$ in PU $(H)$ the projective unitary group of $H$. We shall prove in $\S 14$ that they commute in $\mathrm{U}(H)$.

REMARK 2. In the projective space $(V-\{0\}) / C^{*}$, the intersection of the two 3-surfaces containing $\left(\widehat{R_{1} R_{2}}\right)^{-1}$ and $\widehat{R_{2} R_{3}}$ meet in two complex lines, one meeting the ball $V^{-} / C^{*}$ and one not. As $\arg \varphi^{3}$ passes through the value $-(\pi / 2-\pi / p)$, we get $\eta i \varphi-\eta^{2} \bar{\varphi}^{2}=0$ and the two complex lines coincide. The intersection of $I_{2}$ with the boundary of the ball is thus a single point of tangency for $\bar{\varphi}^{3}=\bar{\eta} i$.

\section{The vertices $p_{i j}, s_{i j}, s_{i j}, t_{i k}$.}

In this and the next two sections, we verify that a region $\Omega(\varphi)$ related to the region $F(\varphi)$ satisfies the codimension-1 condition (CD1). In order to achieve this, we need information about certain $k$-faces of $F(\varphi)$ for $k=0,1,2,3$. The apexes $p_{i j}, i \neq j \in\{1,2,3\}$, and the $k$-faces containing an apex have been discussed in $\S 10$. It remains to discuss those $k$-faces which contain no apex. We do this in stages.

First we define points $s_{i j}, \widetilde{s}_{i j}, t_{i k}$ lying on the intersection of four spinal surfaces containing 3 -faces of $F(\varphi)$. In $\S 13$, we calculate the images of these points under $\gamma$ for the $\hat{\gamma}$ which contain them. In $\S 14.3$, we define the region $\Omega(\varphi)$ and in $\S 14.4$ we verify the (CD1) condition for all its 3 -faces.

The shape of $F(\varphi)$ undergoes a change when $\arg \left(\varphi^{3}\right)$ increases from values less than $\pi / 2-\pi / p$ to greater values. Accordingly, we ultimately consider two cases.

Case 1. $\left|\arg \left(\varphi^{3}\right)\right| \leqq \pi / 2-\pi / p$

Case 2. $\pi / 2-\pi / p \leqq\left|\arg \varphi^{3}\right|<3(\pi / 2-\pi / p)$.

In point of fact, parts of $F(\varphi)$ remain combinatorially unchanged for $-3(\pi / 2-\pi / p)<\arg \varphi^{3}<\pi / 2-\pi / p$, while other parts remain unchanged for $\arg \varphi^{-3}$ in the above range. Define for any $i$ modulo 3 , and for any $\varphi$.

$$
s_{i, i+1}=I_{i} \cap I_{i+1}^{\prime}, \widetilde{s}_{i, i-1}=I_{i} \cap I_{i-1}^{\prime} .
$$

By definition therefore

$$
s_{12}=\left(\widehat{R_{3} R_{1}}\right)^{-1} \cap \widehat{R_{1} R_{2}} \cap\left(\widehat{R_{3} R_{2}}\right)^{-1} \cap \widehat{R_{2} R_{1}} .
$$

The intersection on the right hand side is symmetric under interchange of indices 1 and 2 . Thus we can define $s_{21}=s_{12}$ without contradiction, taking care not to confuse $s_{21}$ with 


$$
{\widetilde{s}}_{21}=\left(\widehat{R_{2} R_{1}}\right)^{-1} \cap \widehat{R_{1} R_{3}} \cap\left(\widehat{R_{1} R_{2}}\right)^{-1} \cap \widehat{R_{2} R_{3}} .
$$

More generally, set

$$
\begin{aligned}
& s_{i j}=\widehat{R_{i} R_{j}} \cap \widehat{R_{j} R_{i}} \cap\left(\widehat{R_{k} R_{i}}\right)^{-1} \cap \widehat{\left(\widehat{R_{k} R_{j}}\right)^{-1}} \\
& \widetilde{s}_{i j}=\left(\widehat{R_{i} R_{j}}\right)^{-1} \cap\left(\widehat{R_{j} R_{i}}\right)^{-1} \cap \widehat{R_{i} R_{k}} \cap \widehat{R_{j} R_{k}} .
\end{aligned}
$$

Also, define for any distinct $i, k$ modulo 3 ,

$$
t_{i k}=I_{i j k} \cap e_{j}^{\perp} \text {. }
$$

Lemma 12.1. Assume $\left|\arg \left(\varphi^{3}\right)\right| \leqq \pi / 2-\pi / p$. Then

$$
\begin{aligned}
s_{12} & =\pi\left(\bar{\eta} i \varphi e_{1}+\bar{\eta} i \bar{\varphi} e_{2}+e_{3}\right) \\
\widetilde{s}_{21} & =\pi\left(-\eta i \varphi e_{1}-\eta i \bar{\varphi} e_{2}+e_{3}\right) \\
t_{32} & =\pi\left(\alpha a(\varphi) \overline{a(\varphi)} e_{1}+\overline{a(\varphi)} e_{2}+a(\varphi) e_{3}\right) \\
t_{23} & =\pi\left(\alpha a(\varphi) \overline{(\varphi)} e_{1}+\overline{a(\bar{\varphi})} e_{2}+\overline{a(\bar{\varphi}) e_{3}}\right)
\end{aligned}
$$

where $\alpha=1 /(2 \sin \pi / p), a(\varphi)=\bar{\varphi}\left(1-\eta i \varphi^{3}\right)$. Moreover, all the above points are in the ball $\pi\left(V^{-}\right)$.

Proof. $\quad$ By $\quad \S 9.2, \quad \kappa\left(R_{i}(\varphi)\right)=R_{i}(\bar{\varphi})^{-1} \quad$ for $\quad i=1,2,3$. Hence $\kappa\left(s_{12}(\varphi)\right)=\widetilde{s}_{21}(\bar{\varphi})$ and $\kappa\left(t_{23}(\varphi)\right)=t_{32}(\bar{\varphi})$. Thus it suffices to verify the lemma for $s_{12}$ and $t_{32}$. By definition $s_{12}=I_{1} \cap I_{2}^{\prime}$. By Lemma 11.2, the equation of the preimage of $I_{2}(\varphi)$ is:

$$
I_{2}(\varphi): \quad x_{3}-\bar{\eta} i \bar{\varphi} x_{1}=0 .
$$

Applying $J^{-1}$, we get as the equation for $I_{1}$ :

$$
I_{1}(\varphi): \quad x_{2}-\bar{\eta} i \bar{\varphi} x_{3}=0 .
$$

By (11.2), $I_{2}^{\prime}(\varphi)=J^{\prime} I_{2}(\bar{\varphi})$. Hence the equation of the preimage of $I_{2}^{\prime}(\varphi)$ is given by

$$
I_{2}^{\prime}(\varphi): \quad x_{1}-\bar{\eta} i \varphi x_{3}=0 .
$$

Hence a preimage of $s_{12}(\varphi)$ is given by

$$
x_{1}=\bar{\eta} i \bar{\varphi}, x_{2}=\bar{\eta} i \varphi, x_{3}=1 \text {, }
$$

verifying the assertion for $s_{12}$.

As for $t_{32}$, by definition

$$
t_{32}=I_{312} \cap e_{1}^{\perp} .
$$

The equation of $I_{312}$ is given by (12.1.1):

$$
I_{1}: \quad x_{2}-\bar{\eta} i \bar{\varphi} x_{3}=0 .
$$

By (10.1.1), the equation for $e_{1}^{\perp}$ is 


$$
e_{1}^{\perp}: \quad x_{1}-\alpha \bar{\varphi} x_{2}-\alpha \varphi x_{3}=0 .
$$

Solving these two equations simultaneously yields

$$
\begin{aligned}
\pi^{-1}\left(t_{32}\right) & =x_{3}\left(\alpha \varphi\left(\bar{\eta} i \bar{\varphi}^{3}+1\right), \bar{\eta} i \bar{\varphi}, 1\right) \\
& =x\left(\alpha \left(2+\bar{\eta} i \bar{\varphi}^{3}-\eta i \varphi^{3}, \varphi\left(1+\bar{\eta} i \bar{\varphi}^{3}\right), \bar{\varphi}\left(1-\eta i \varphi^{3}\right)\right.\right. \\
& =x(\alpha a \bar{a}, \bar{a}, a)
\end{aligned}
$$

where $a=a(\varphi)=\bar{\varphi}\left(1-\eta i \varphi^{3}\right)$ and $x \in C-\{0\}$.

It remains only to verify that the points are in the ball $\pi\left(V^{-}\right)$.

Notation. For convenience, we shall denote by $s_{i j}$ and $\widetilde{s}_{i}$, vectors in $V$. For example, we write

$$
s_{12}=\bar{\eta} i \varphi e_{1}+\bar{\eta} i \bar{\varphi} e_{2}+e_{3}, s_{21}=-\eta i \varphi e_{1}-\eta i \bar{\varphi} e_{2}+e_{3}
$$

and similarly we sometimes denote by $t_{i k}$ the vector in $V$ representing it as in the formula of Lemma 12.1. When there is risk of confusion, we write $s_{i j}$ (in the ball) to indicate $\pi\left(s_{i j}\right)$.

For the vector $s_{12}$,

$$
\begin{aligned}
\left\langle s_{12}, s_{12}\right\rangle= & 3-\frac{i}{\eta-\eta}[\bar{\eta} i \varphi(-\eta i \varphi \cdot \varphi+\bar{\varphi})+\bar{\eta} i \bar{\varphi}(-\eta i \bar{\varphi} \cdot \bar{\varphi}+\varphi) \\
& +(-\eta i \bar{\varphi} \cdot \varphi-\eta i \varphi \cdot \bar{\varphi})] \\
= & 3-\frac{i}{\eta-\bar{\eta}}\left[-2 i(\eta-\bar{\eta})+\varphi^{3}+\bar{\varphi}^{3}\right] \\
= & 1-\frac{i}{\eta-\bar{\eta}}\left[\phi^{3}+\bar{\varphi}^{3}\right] \\
= & 1-\frac{\cos \arg \varphi^{3}}{\sin \frac{\pi}{p}} .
\end{aligned}
$$

Thus $\left\langle s_{12}, s_{12}\right\rangle<0$ if and only if $\left|\arg \varphi^{3}\right|<\pi / 2-\pi / p$. Since $\kappa\left(s_{12}(\varphi)\right)=$ $\widetilde{s}_{21}(\bar{\varphi})$, we see that $\left\langle\widetilde{s}_{21}, \widetilde{s}_{21}\right\rangle=\left\langle s_{12}, s_{12}\right\rangle$ and thus $\widetilde{s}_{21} \in \pi\left(V^{-}\right)$for $\left|\arg \varphi^{3}\right|<\pi / 2-\pi / p$.

Before verifying that $t_{32} \in V^{-}$, we note the identity

$$
\begin{aligned}
a^{2} \bar{\varphi}+\bar{a}^{2} \varphi & =\bar{\varphi}^{3}\left(1-2 \eta i \varphi^{3}-\eta^{2} \varphi^{6}\right)+\varphi^{3}\left(1+2 \bar{\eta} i \bar{\varphi}^{3}-\bar{\eta}^{2} \bar{\varphi}^{6}\right) \\
& =-2 \eta i+2 \bar{\eta} i+\left(1-\eta^{2}\right) \varphi^{3}+\left(1-\bar{\eta}^{2}\right) \bar{\varphi}^{3} .
\end{aligned}
$$

Hence

$$
\begin{aligned}
\alpha\left(a^{2} \bar{\varphi}+\bar{a}^{2} \varphi\right) & =\frac{i}{\eta-\bar{\eta}}\left(2 \frac{\eta-\bar{\eta}}{i}+\eta(\bar{\eta}-\eta) \varphi^{3}+\bar{\eta}(\eta-\bar{\eta}) \bar{\varphi}^{3}\right) \\
& =2-\eta i \varphi^{3}+\bar{\eta} i \bar{\varphi}^{3} \\
& =a \bar{a} .
\end{aligned}
$$


Then

$$
\begin{aligned}
\left\langle t_{32}, t_{32}\right\rangle= & \left\langle\alpha a \bar{a} e_{1}+\bar{a} e_{2}+a e_{3}, \alpha a \bar{a} e_{1}+\bar{a} e_{2}+a e_{3}\right\rangle \\
= & \alpha a \bar{a}[\alpha a \bar{a}-\alpha \varphi a-\alpha \bar{\varphi} \bar{a}] \\
& \quad+\bar{a}\left[a-\alpha^{2} a \bar{a} \bar{\varphi}-\alpha \bar{a} \varphi\right] \\
& \quad+a\left[\bar{a}-\alpha^{2} a \bar{a} \varphi-\alpha a \bar{\varphi}\right] \\
= & 2 a \bar{a}-\alpha^{9} a \bar{a}-\alpha\left(a^{2} \bar{\varphi}+\bar{a}^{2} \varphi\right) \\
= & a \bar{a}\left(1-\alpha^{2} a \bar{a}\right)+a \bar{a}-\alpha\left(a^{2} \bar{\varphi}+\bar{a}^{2} \varphi\right) \\
= & a \bar{a}\left(1-\alpha^{2} \alpha \bar{a}\right) .
\end{aligned}
$$

Thus $\left\langle t_{32}, t_{32}\right\rangle<0$ if and only if $1-\alpha^{2} a \bar{a}<0$; that is, $\alpha^{-2}<a \bar{a}$. This is equivalent to

$$
\begin{aligned}
& \left(\frac{\eta-\bar{\eta}}{i}\right)^{2}<\left(1-\eta i \varphi^{3}\right)\left(1+\bar{\eta} i \varphi^{3}\right) \\
& 2-\eta^{2}-\bar{\eta}^{2}<2-\eta i \varphi^{3}+\bar{\eta} i \bar{\varphi}^{3} \\
& -2 \cos \frac{2 \pi}{p}<2 \sin \left(\frac{\pi}{p}+3 \theta\right), \quad \theta=\arg \varphi \\
& -\sin \left(\frac{\pi}{2}-\frac{2 \pi}{p}\right)<\sin \left(\frac{\pi}{p}+3 \theta\right) \\
& -\left(\frac{\pi}{2}-\frac{\pi}{p}\right)<3 \theta .
\end{aligned}
$$

Thus $t_{32} \in V^{-}$for $-(\pi / 2-\pi / p)<\arg \phi^{3}<3(\pi / 2-\pi / p)$. Applying $t_{23}(\varphi)=\kappa\left(t_{32}(\bar{\varphi})\right)$, we find that $t_{23} \in V^{-}$for $-3(\pi / 2-\pi / p)<\arg \varphi^{3}<$ $\pi / 2-\pi / p$.

The next lemma will allow us to determine how the cell complex $F(\phi)$ changes as $\arg \phi^{3}$ attains the values $\pi / 2-\pi / p$.

Lemma 12.2. For $\left|\arg \left(\varphi^{3}\right)\right| \leqq \pi / 2-\pi / p$, and for any integer $i$ modulo 3 ,

(i ) $s_{i, i+1}=\bar{\eta} \sqrt{-1} \varphi e_{i}+\bar{\eta} \sqrt{-1} \bar{\varphi} e_{i+1}+e_{i-1}$

$$
\begin{aligned}
& \widetilde{s}_{i+1, i}=-\eta \sqrt{-1} \varphi e_{i}-\eta \sqrt{-1} \bar{\varphi} e_{i+1}+e_{i-1} \\
& t_{i, i-1}=a(\varphi) e_{i}+\bar{a}(\varphi) e_{i-1}+\alpha a(\varphi) \bar{a}(\varphi) e_{i+1} \\
& t_{i, i+1}=a(\bar{\varphi}) e_{i}+\bar{a}(\bar{\varphi}) e_{i+1}+\alpha a(\bar{\varphi}) \bar{a}(\bar{\varphi}) e_{i-1}
\end{aligned}
$$

where $\alpha=(2 \sin \pi / p)^{-1}, a(\varphi)=\bar{\varphi}\left(1-\eta i \varphi^{3}\right)$, and $\bar{a}(\varphi)=\overline{a(\varphi)}$.

(ii) $s_{i, i+1}=\widetilde{s}_{i, i-1}$ if and only if $\arg \phi^{3}=-(\pi / 2-\pi / p)$

$$
s_{i, i+1}=\widetilde{s}_{i-1, i+1} \text { if and only if arg } \varphi^{3}=\pi / 2-\pi / p
$$

(iii) $s_{i, i+1}=t_{i-1, i+1}$ if and only if $\arg \varphi^{3}=-(\pi / 2-\pi / p)$

$$
\widetilde{s}_{i+1, i}=t_{i+1, i-1} \text { if and only if } \arg \varphi^{3}=\pi / 2-\pi / p \text {. }
$$

Proof. By Lemma 12.2

$$
\widetilde{s}_{13}=\pi\left(-\eta i \bar{\varphi} e_{1}+e_{2}-\eta i \varphi e_{3}\right)
$$


and $s_{12}=\pi\left(\bar{\eta} i \varphi e_{1}+\bar{\eta} i \bar{\varphi} e_{2}+e_{3}\right)=\pi\left(\eta^{2} e_{1}+e_{2}-\eta i \varphi e_{3}\right)$. Thus $s_{12}=\widetilde{s}_{13}$ if and only if $\varphi^{3}=-\eta i$. By applying $J$ and $J^{-1}$, we get the first assertion of (i), and by applying $\kappa$ one gets the second.

As for the remaining values of $\varphi$, the next lemma gives an explicit formula for the points $s_{i j}, \widetilde{s}_{i j}$, and $t_{i k}$ in terms of the vector $v_{i j k}$ of $V$ listed in $\S 9.1$.

LEMMA 12.3. Assume that $\pi / 2-\pi / p \leqq\left|\arg \varphi^{3}\right|<3(\pi / 2-\pi / p)$. Then for any permutation ijk of $(1,2,3)$,

$$
\begin{aligned}
s_{i j} & =\left\{\begin{array}{l}
v_{k i j} \text { if }\left\langle v_{k i j}, v_{k i j}\right\rangle<0 \\
v_{k j i} \text { if }\left\langle v_{k j i}, v_{k j i}\right\rangle<0
\end{array}\right. \\
\widetilde{s}_{i j} & =\left\{\begin{array}{l}
v_{i j k} \text { if }\left\langle v_{i j k}, v_{i j k}\right\rangle<0 \\
v_{j i k} \text { if }\left\langle v_{j i k}, v_{j i k}\right\rangle<0
\end{array}\right. \\
t_{i k} & =\left\{\begin{array}{l}
v_{i j k} \text { if }\left\langle v_{i j k}, v_{i j k}\right\rangle<0 \\
\text { by the formula }(12.2) \text { if }\left\langle v_{i j k}, v_{i j k}\right\rangle>0 .
\end{array}\right.
\end{aligned}
$$

That is,

$$
\begin{array}{r}
s_{12}=\widetilde{s}_{13}=t_{32}=v_{312} \\
\text { for }-3\left(\frac{\pi}{2}-\frac{\pi}{p}\right)<\arg \phi^{3} \leqq-\left(\frac{\pi}{2}-\frac{\pi}{p}\right) \\
\widetilde{s}_{21}=s_{31}=t_{23}=v_{213} \\
\text { for } \quad 3\left(\frac{\pi}{2}-\frac{\pi}{p}\right)>\arg \varphi^{3} \geqq 3\left(\frac{\pi}{2}-\frac{\pi}{p}\right) .
\end{array}
$$

Proof. The computation of the indicated points in the indicated range for $\varphi$ proceeds from equation (11.3.b) for $I_{i j k}$, and the verification entails straightforward solutions of two linear equations. The condition on $\varphi$ that $v_{i j k_{k}} \in V^{-}$can be read off (9.1.4) and (9.1.5).

REMARK 1. For $|\arg \varphi| \geqq \pi / 2-\pi / p, \quad \widetilde{s}_{k i}=s_{i j}$ if and only if $\left\langle v_{i j k}, v_{i j k}\right\rangle \leqq 0$.

REMARK 2. As a consequence of the given relations in the group $\Gamma$, we will see in $\S 14$ that $\left\langle v_{i j k}, v_{i k j}\right\rangle=0=\left\langle v_{i j k}, v_{j i k}\right\rangle$. This can be verified directly of course. Since $H$ has signature (two positive, one negative) $\left\langle v_{i j k}, v_{i j k}\right\rangle\left\langle 0\right.$ implies $\left.\left\langle v_{j i k}, v_{j i k}\right\rangle=\left\langle v_{i k j}, v_{i k j}\right\rangle\right\rangle 0$. If $\left\langle v_{i j k}, v_{i j k}\right\rangle=0$, then $v_{i j k} \in C v_{i k j}+C v_{j i k}$.

13. Towards verification of (CD1): images of points.

LEMMA 13.1. For all distinct $i, j$ and all values of $\varphi$ with $\left|\arg \varphi^{3}\right|<3(\pi / 2-\pi / p)$ 


$$
\pi\left(R_{i} s_{i j}\right)=\pi\left(\widetilde{s}_{i k}\right)
$$

Proof. By symmetry, it suffices to prove that

$$
\pi\left(R_{1} s_{12}\right)=\pi\left(\widetilde{s}_{13}\right) .
$$

If $\left|\arg \varphi^{3}\right| \leqq \pi / 2-\pi / p$, then by Lemma $12.1, s_{12}=\bar{\eta} i \varphi e_{1}+\bar{\eta} i \bar{\varphi} e_{2}+$ $e_{3}$ and the components of $R_{1} s_{12}$ are given by

$$
\left(\begin{array}{ccc}
\eta^{2} & -\eta i \bar{\varphi} & -\eta i \varphi \\
0 & 1 & 0 \\
0 & 0 & 1
\end{array}\right)\left(\begin{array}{c}
\bar{\eta} i \varphi \\
\bar{\eta} i \bar{\varphi} \\
1
\end{array}\right)=\left(\begin{array}{c}
\bar{\varphi}^{2} \\
\bar{\eta} i \bar{\varphi} \\
1
\end{array}\right)=\bar{\eta} i \bar{\varphi}\left(\begin{array}{c}
-\eta i \bar{\varphi} \\
1 \\
\eta i \varphi
\end{array}\right)
$$

so that $\pi\left(R_{1} s_{12}\right)=\pi\left(\widetilde{s}_{13}\right)$.

If $\pi / 2-\pi / p \leqq \arg \varphi^{3}<3(\pi / 2-\pi / p)$, then by Lemma 12.3, $s_{12}=$ $v_{321}$ since $\left\langle v_{321}, v_{321}\right\rangle=\left\langle v_{213}, v_{213}\right\rangle<0$. Thus $R_{1} s_{12}$ is given by (cf. $\S 9.1$ for $v_{321}$ and $\left.v_{132}\right)$.

$$
\left(\begin{array}{ccc}
\eta^{2} & -\eta i \bar{\varphi} & -\eta i \phi \\
0 & 1 & 0 \\
0 & 0 & 1
\end{array}\right)\left(\begin{array}{c}
\bar{\eta} i \bar{\varphi} \\
1 \\
-\eta i \varphi
\end{array}\right)=\left(\begin{array}{c}
-\eta^{2} \varphi^{2} \\
1 \\
-\eta i \varphi
\end{array}\right)=-\eta i \varphi\left(\begin{array}{c}
-\eta i \varphi \\
\bar{\eta} i \bar{\varphi} \\
1
\end{array}\right) .
$$

Thus $R_{1} s_{12}=-\eta i \varphi v_{132}=-\eta i \varphi \widetilde{s}_{13}$ and again $\pi\left(R_{1} s_{12}\right)=\pi\left(\widetilde{s}_{13}\right)$. The result for $-3(\pi / 2-\pi / p)<\arg \phi^{3} \leqq \pi / 2-\pi / p$ can be deduced from the above by applying the isometry $J^{\prime}: V(\mathscr{P}) \rightarrow V(\bar{\varphi})$.

Lemma 13.2. For all distinct $i, j$ and for all $\varphi$ with $\left|\arg \varphi^{3}\right|<$ $3(\pi / 2-\pi / p)$

$$
\pi\left(R_{k} \widetilde{s}_{j \imath}\right)=\pi\left(s_{\imath j}\right)
$$

Proof. It suffices to prove the result for $R_{3} \widetilde{s}_{21}$. If $\left|\arg \varphi^{3}\right|<$ $\pi / 2-\pi / p$, the computation is

$$
\left(\begin{array}{ccc}
1 & 0 & 0 \\
0 & 1 & 0 \\
-\eta i \bar{\varphi} & -\eta i \varphi & \eta^{2}
\end{array}\right)\left(\begin{array}{c}
-\eta i \varphi \\
-\eta i \bar{\varphi} \\
1
\end{array}\right)=\left(\begin{array}{c}
-\eta i \varphi \\
-\eta i \bar{\varphi} \\
-\eta^{2}
\end{array}\right)=-\eta^{2}\left(\begin{array}{c}
\bar{\eta} i \varphi \\
\bar{\eta} i \bar{\varphi} \\
1
\end{array}\right) .
$$

If $\pi / 2-\pi / p \leqq \arg \varphi^{3}<3(\pi / 2-\pi / p)$, then by Lemma 12.3, $\widetilde{s}_{21}=v_{213}$ and $s_{12}=v_{321}$. The computation is (cf. $\S 9.1$ ).

$$
\left(\begin{array}{ccc}
1 & 0 & 0 \\
0 & 1 & 0 \\
-\eta i \bar{\varphi} & -\eta i \varphi & \eta^{2}
\end{array}\right)\left(\begin{array}{c}
1 \\
-\eta i \varphi \\
\bar{\eta} i \bar{\varphi}
\end{array}\right)=\left(\begin{array}{c}
1 \\
-\eta i \varphi \\
-\eta^{2} \varphi^{2}
\end{array}\right)=-\eta i \varphi\left(\begin{array}{c}
\overline{\bar{\varphi}} \bar{\varphi} \\
1 \\
-\eta i \varphi
\end{array}\right) .
$$

For the remaining values of $\varphi$, the result can be deduced from the foregoing by means of the isometry $J^{\prime}: V(\varphi) \rightarrow V(\bar{\varphi})$. 
The information in Lemmas 13.1 and 13.2 yields the hexagonal diagram

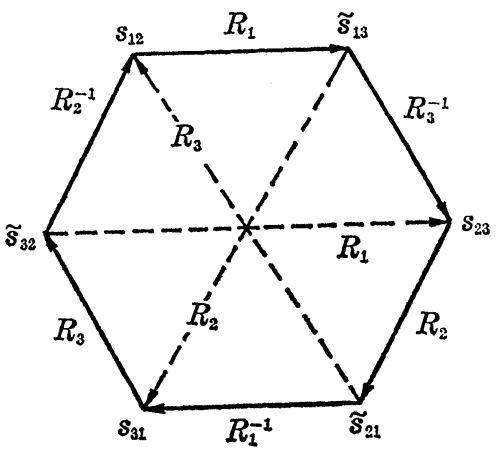

Lemma 13.3. (i ) For each index $i$ modulo 3,

$$
\begin{aligned}
& R_{i} R_{i+1}=J \text { on } I_{i} \text { if } s_{i, i+1} \neq \widetilde{s}_{i, i-1} \\
& R_{i+1} R_{i}=J^{-1} \text { on } I_{i+1}^{\prime} \text { if } s_{i, i+1} \neq \widetilde{s}_{i-1, i+1}
\end{aligned}
$$

(ii) $\left(R_{i} R_{j} R_{k}\right)^{2}$ fixes each point of $I_{i j k}$ if

$$
\left\langle v_{i j k}, v_{i j k}\right\rangle>0
$$

(iii) $R_{k} R_{i} R_{j} R_{i} s_{i j}=s_{i j}$ (in the ball)

(all permutations $(i, j, k)$ of $(1,2,3)$ in (ii) and (iii)).

Proof. (i) By symmetry, it suffices to consider the case $i=1$. Then by definition (12.1),

$$
s_{12}=I_{1} \cap I_{2}^{\prime}, \widetilde{s}_{13}=I_{1} \cap I_{3}^{\prime} .
$$

By the hypothesis $s_{12} \neq \widetilde{s}_{13}, I_{1}$ is the unique $C$-line containing $s_{12}$ and $\widetilde{s}_{13}$. From the hexagonal diagram,

$$
R_{1} R_{2} s_{12}=s_{23}, R_{1} R_{2} \widetilde{s}_{13}=\widetilde{s}_{21} .
$$

Hence $R_{1} R_{2} I_{1}=I_{2}$, the unique $C$-line containing $s_{23}$ and $\widetilde{s}_{21}$. Since the restrictions of $R_{1} R_{2}$ and $J$ to $I_{1}$ are isometries, which coincide on $s_{12}$ and $\widetilde{s}_{13}$, the isometry $J^{-1} R_{1} R_{2}$ fixes these two points and therefore every point of the geodesic line joining them, and therefore every point of the $C$-line joining them. It follows that $R_{1} R_{2}$ and $J$ coincide on $I_{1}$. That $R_{2} R_{1}=J^{-1}$ on $I_{1}^{\prime}$ comes from applying the complex conjugation isometry $\kappa_{2}: V(\bar{\varphi}) \rightarrow V(\varphi)$ :

$$
\begin{aligned}
& \kappa\left(s_{12}(\bar{\varphi})\right)=\widetilde{s}_{21}(\varphi), \kappa\left(\widetilde{s}_{13}(\bar{\varphi})\right)=s_{31}(\varphi) \\
& \kappa\left(R_{1}(\bar{\varphi})\right)=R_{1}^{-1}(\varphi), \kappa\left(R_{2}(\bar{\varphi})\right)=R_{2}^{-1}(\varphi) \\
& \kappa\left(I_{1}(\bar{\varphi})\right)=I_{1}^{\prime}(\varphi) .
\end{aligned}
$$


At $\bar{\varphi}, R_{1} R_{2} s_{12}=s_{23}, R_{1} R_{2} \widetilde{s}_{13}=\widetilde{s}_{21}$. Applying $\kappa$, we get at $\mathscr{P}: R_{1}^{-1} R_{2}^{-1} \widetilde{\mathcal{S}}_{21}=$ $\widetilde{s}_{32}, R_{1}^{-1} R_{2}^{-1} s_{31}=s_{12}$. Hence $R_{2} R_{1} \widetilde{s}_{32}=\widetilde{s}_{21}, R_{2} R_{1} s_{12}=s_{31}$. It follows that $R_{2} R_{1}$ coincides with $J^{-1}$ on $I_{2}^{\prime}$. If $\left\langle v_{123}, v_{123}\right\rangle>0\left(\right.$ resp. $\left.\left\langle v_{321}, v_{321}\right\rangle>0\right)$, we can apply Remark 1 following Lemma 12.3. The information in (i) then yields the triangular diagrams
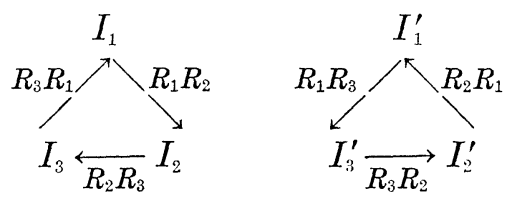

On $I_{2}, R_{1} R_{2} \cdot R_{3} R_{1} \cdot R_{23}=J^{3}=$ identity, so that $\left(R_{1} R_{2} R_{3}\right)^{2}$ fixes each point of $I_{2}$ if $\left\langle v_{123}, v_{123}\right\rangle>0$. Similarly $\left(R_{3} R_{2} R_{1}\right)^{2}$ fixes each point of $I_{2}^{\prime}$ if $\left\langle v_{321}, v_{321}\right\rangle>0$.

(iii) follows at once from the hexagonal diagram.

REMARK. Assertion (ii) of Lemma 13.3 has been pointed out before in Remark 1 following Lemma 11.2; it comes from the fact that $v_{\imath j_{k}}$ is the eigenvector of $\left(R_{i} R_{j} R_{k}\right)^{2}$ corresponding to the eigenvalue of multiplicity 1 . Thus $v_{i j k}^{\perp} \cap V^{-}$is not empty if and only if $\left\langle v_{i j k}, v_{i j_{k}}\right\rangle>0$.

Consequences in another direction of the information in the hexagonal diagram are given in the next lemma.

Lemma 13.4. For any distinct $i, j$ from $\{1,2,3\}$,

$$
\begin{aligned}
& s_{i j}=\hat{R}_{i} \cap \hat{R}_{j} \cap R_{i} \hat{R}_{j} \cap \widehat{R_{j} R_{i}} \cap R_{i} \hat{R}_{j} R_{i} \cap \hat{R}_{k}^{-1} \cap\left(\widehat{R_{k} R_{i}}\right)^{-1} \cap \widehat{\left(\widehat{R_{k} R_{j}}\right)^{-1}} \\
& \left.\widetilde{s}_{i j}=\hat{R}_{i}^{-1} \cap \widehat{R}_{j}^{-1} \cap \widehat{\left(R_{i} R_{j}\right.}\right)^{-1} \cap\left(R_{j} \hat{R}_{i}\right)^{-1} \cap\left(R_{i} \widehat{R}_{j} R_{i}\right)^{-1} \cap \widehat{R_{k}} \cap \widehat{R_{i} R_{k}} \cap \widehat{R_{j} R_{k}} \\
& t_{i k}=\widehat{R}_{j} \cap \hat{R}_{j}^{-1} \cap \widehat{R_{j} R_{k}} \cap\left(R_{i} \hat{R}_{j}\right)^{-1} \cap R_{j} \hat{R}_{k} R_{j} \cap\left(R_{j} \widehat{R}_{i} R_{j}\right)^{-1} .
\end{aligned}
$$

Proof. The definition of the above points are given in (12.1) and (12.2), explicit formulae for them being given in Lemmas 12.2 and 12.3 .

It is easy to verify that the number $\left|\left\langle p_{0}, s_{i j}\right\rangle\right|$ is invariant under the cyclic permutation automorphism $J$ and also under the isometries $J^{\prime}$ and $\kappa$ of $V(\varphi)$ to $V(\bar{\varphi})$ and hence under the group Isom $F$ of the six isometries of $V(\varphi)$ to $V(\varphi)$ generated by $J$ and $a_{12}$ (cf. $\S 10.2$ ). Under Isom $F$, the six points $\left\{s_{i j}, \widetilde{s}_{j i} ; i \neq 1\right\}$ are permuted transitively and the point $p_{0}$ is fixed. Hence for any permutation $(i j k)$ of $(1,2,3)$,

$$
d\left(p_{0}, s_{i j}\right)=d\left(p_{0}, \widetilde{s}_{i k}\right) .
$$

By Lemma 13.1, $R_{i} s_{i j}=\widetilde{s}_{i k}$ (in the ball). Hence $d\left(\widetilde{s}_{i j}, p_{0}\right)=d\left(s_{i k}, p_{0}\right)=$ $d\left(R_{i} s_{i j}, p_{0}\right)$. By definition therefore, $s_{i j} \in \hat{R}_{i}$. By symmetry $s_{i j} \in \hat{R}_{j}$. 
The proof $s_{i j} \in \hat{R}_{k}^{-1}$ is similar. Also

$$
R_{j} R_{i} s_{i j}=R_{j} \widetilde{s}_{i k}=s_{k i}
$$

so that $d\left(s_{i j}, p_{0}\right)=d\left(s_{k i}, p_{0}\right)=d\left(R_{j} R_{i} s_{i j}, p_{0}\right)$. Hence $s_{i j} \in \widehat{R_{j} R_{i}}$. Also

$$
R_{i} R_{j} R_{i} s_{i j}=R_{i}\left(s_{k i}\right)=R_{i}\left(s_{i k}\right)=\widetilde{s}_{i j}
$$

so that $d\left(s_{i j}, p_{0}\right)=d\left(\widetilde{s}_{i j}, p_{0}\right)=d\left(R_{i} R_{j} R_{i} s_{i j}, p_{0}\right)$. Hence $s_{i j} \in \widehat{R_{i} R_{j} R_{i}}$. The remaining assertions for $s_{i j}$ and $\widetilde{s}_{i j}$ result from applying Isom $F$ to the above results:

As for $t_{i k}$, by definition,

$$
t_{i k}=I_{i j k} \cap e_{j}^{\perp}=\hat{R}_{i} \cap \hat{R}_{j}^{-1} \cap R_{j} R_{k} \cap\left(\widehat{R_{i} R_{j}}\right)^{-1} .
$$

Since $R_{j}$ fixes each point of $e_{j}^{\perp}, R_{j} R_{k} R_{j}\left(t_{i k}\right)=R_{j} R_{k} t_{i k}$. If $\left.\left\langle v_{i j k}, v_{i j k}\right\rangle\right\rangle$ 0 , then $I_{i j k}$ is given by (11.3a) and Lemma 13.3 (i) yields $R_{j} R_{k} t_{i k}=$ $t_{j i}$. Thus

$$
d\left(R_{j} R_{k} R_{j} t_{i k}, p_{0}\right)=d\left(t_{j i}, p_{0}\right)=d\left(t_{i k}, p_{0}\right)
$$

and $t_{i k} \in R_{j} \widehat{R_{k} R_{j}}$. On the other hand, if $\left\langle v_{i j k}, v_{i j k}\right\rangle<0$, then $t_{i k}=$ $v_{i j k}=s_{j k}$, and

$$
R_{j} R_{k} t_{i k}=R_{j} R_{k} \widetilde{s}_{j k}=R_{j} s_{k i}=s_{i k}=v_{j k i}=t_{j i} .
$$

Thus by the same argument, $t_{i k} \in R_{j} \widehat{R_{k}} R_{j}$ is this case too. That $t_{\imath k} \in\left(R_{j} \hat{R}_{i} R_{j}\right)^{-1}$ can be deduced from the foregoing by applying the isometry $a_{i k}$. The proof of Lemma 13.4 is now complete.

Lemma 13.5. For all permutation ijk of $(1,2,3)$ and for all $\varphi$ with $\left|\arg \varphi^{3}\right|<3(\pi / 2-\pi / p)$

$$
R_{j} R_{k} t_{i k}=t_{j i} \quad(\text { in the ball }) .
$$

Proof. This was demonstrated in the proof of Lemma 13.4.

14. Some identities in $\Gamma$, some lines in $F$, and (CD1) for $\Omega(\varphi)$.

14.1. Relations in $\Gamma$. We mentioned in Remark 2 following Lemma 12.3 that $\left\langle v_{i j k}, v_{j i k}\right\rangle=0=\left\langle v_{i j k}, v_{i k j}\right\rangle$ for all permutations $(i j k)$ of $(1,2,3)$. The group relations proved in the next lemma explain these orthogonality relations and the relation $\left\langle e_{j}, v_{i j k}\right\rangle=0$ as well; they arise from the relation $R_{i} R_{j} R_{i}=R_{j} R_{i} R_{j}$.

Lemma 14.1. For any permutation (ijk) of $(1,2,3)$ and for all $\varphi$, (i) $R_{j}\left(R_{i} R_{j} R_{k}\right)^{2}=\left(R_{i} R_{j} R_{k}\right)^{2} R_{j}$. 
(ii) Let $a=R_{i} R_{j} R_{k} R_{j}, b=R_{j}^{-1} R_{i} R_{j} R_{k}, c=R_{k}^{-1} R_{i} R_{k} R_{j}$ and $I^{\prime \prime}=$ $\{\{a, b\}\}$, the subgroup of $\Gamma$ generated by $\{a, b\}$. Then $\Gamma^{\prime}$ is abelian.

(iii) $\left(R_{i} R_{j} R_{k}\right)^{2}$ and $\left(R_{i} R_{k} R_{j}\right)^{2}$ commute.

(iv) $\left(R_{i} R_{j} R_{k}\right)^{2}$ and $\left(R_{j} R_{i} R_{k}\right)^{2}$ commute.

( v ) $\quad a^{3}=\left(R_{i} R_{j} R_{k_{k}}\right)^{2} \cdot\left(R_{i} R_{k} R_{j}\right)^{2}$.

Proof. $\quad R_{j} R_{\imath} R_{j} R_{k} R_{\imath} R_{j} R_{k}=R_{i} R_{j} R_{\imath} R_{k} R_{\imath} R_{j} R_{k}$

$=R_{i} R_{j} R_{k} R_{i} R_{k} R_{j} R_{k}$

$=R_{\imath} R_{j} R_{k} R_{i} R_{j} R_{k} R_{j}$.

This proves (i).

By (i) $a b=\left(R_{\imath} R_{j} R_{k}\right)^{2}=b a$. This proves (ii).

$$
\begin{aligned}
c a & =R_{k}^{-1} R_{\imath} R_{k} R_{j} R_{i} R_{j} R_{k} R_{\jmath} \\
& =R_{k}^{-1} R_{i} R_{k} R_{j} R_{i} R_{k} R_{j} R_{k} \\
& =\left(R_{i} R_{k} R_{j}\right)^{2}, \text { by (i). }
\end{aligned}
$$

Moreover $b^{-1} a=R_{k}^{-1} R_{j}^{-1} R_{i}^{-1} R_{j} R_{i} R_{j} R_{k} R_{j}=R_{k}^{-1} R_{j}^{-1} R_{i}^{-1} \cdot R_{\imath} R_{j} R_{\imath} R_{k} R_{\text {, }}=$ $R_{k}^{-1} R_{i} R_{k} R_{j}=c$. Hence $\{\{a, b\}\}=\{a, c\}$ and $\left(R_{i} R_{k} R_{j}\right)^{2} \in \Gamma^{\prime}$. This and (ii) imply (iii). Applying the isomorphism $\kappa: \Gamma(\bar{\varphi}) \rightarrow \Gamma(\phi)$ to the relation (iii) in $\Gamma(\bar{\varphi})$ yields that $\left(R_{i}^{-1} R_{j}^{-1} R_{k}^{-1}\right)^{2}$ and $\left(R_{i}^{-1} R_{k}^{-1} R_{j}^{-1}\right)^{2}$ com-

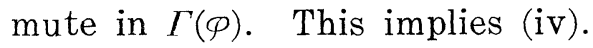

We have from above

$$
\left(R_{i} R_{j} R_{k}\right)^{2}\left(R_{i} R_{k} R_{j}\right)^{2}=a b c a=a b\left(b^{-1} a\right) a=a^{3} .
$$

This proves (iii).

\subsection{Geodesic lines.}

LEMMA 14.2.1. Assume $-(\pi / 2-\pi / p)<\arg \mathcal{P}^{3}<3(\pi / 2-\pi / p)$. For all $i$,

(i ) $I_{\imath} \cap \hat{R}_{i}$ and $I_{i} \cap \hat{R}_{i}^{-1}$ are geodesic lines.

(ii) $e_{i} \cap \widehat{R}_{i}^{R_{\imath+1}}$ and $e_{i} \cap\left(\widehat{R_{\imath-1}} R_{i}\right)^{-1}$ are geodesic lines.

Proof. $\quad I_{i}$ is a slice of $\widehat{R_{i} R_{i+1}}$ by Lemma 11.1. Clearly $e_{i}$ is a slice of $\hat{R}_{i}$. We have $e_{i}$ is orthogonal to $I_{i}$ at $t_{\imath-1, \imath+1}$; for by Lemma $11.2(\mathrm{i}), v_{123}$ is the normal to $I_{2}$, and $\left\langle e_{2}, v_{123}\right\rangle=0$ as is easily verified. Hence $I_{i} \cap \hat{R}_{2}$ is a geodesic line by Lemma 3.2.5. The same argument applies to $I_{i} \widehat{\cap R_{\imath}^{-1}}$. This proves (i).

Since the $C$-line $e_{i}$ is orthogonal to the slice $I_{i}=\left(\widehat{R_{i-1}} R_{\imath}\right)^{-1} \cap$ $R_{i} \hat{R}_{1+1}$, (ii) also follows from Lemma 3.2.5.

REMARK 1. For $-3(\pi / 2-\pi / p)<\arg \varphi^{3}<\pi / 2-\pi / p$, we get by 
the map $J^{\prime}: V(\bar{\varphi}) \rightarrow V(\varphi)$, that:

(i $)^{\prime} \quad I_{i}^{\prime} \cap \hat{R}_{i}$ and $I_{i}^{\prime} \cap \hat{R}_{i}^{-1}$ are geodesic lines.

(ii $)^{\prime} \quad e_{i}^{\perp} \cap \widehat{R_{i} R_{i-1}}$ and $I_{i}^{\prime} \cap\left(\widehat{R_{i+1}} \widehat{R_{i}}\right)^{-1}$ are geodesic lines.

Assertions (ii) and (ii) are in fact valid for $\left|\arg \varphi^{3}\right|<3(\pi / 2-\pi / p)$. For $e_{i}^{\llcorner} \cap \widehat{R_{1} R_{2}}$ is real analytic curve in the ball depending analytically on the parameter $\varphi$ throughout the interval $\left|\arg \varphi^{3}\right|<3(\pi / 2-\pi / p)$. Since it is a geodesic line on an open subinterval of $\varphi$, and since the condition of being a geodesic line is real analytic (with respect to standard nonhomogeneous coordinates centered at $p_{12}$, it amounts to being a real line), it follows that $e_{1}^{\perp} \cap R_{1} R_{2}$ is a geodesic line for all $\varphi$ with $\left|\arg \varphi^{3}\right|<3(\pi / 2-\pi / p)$.

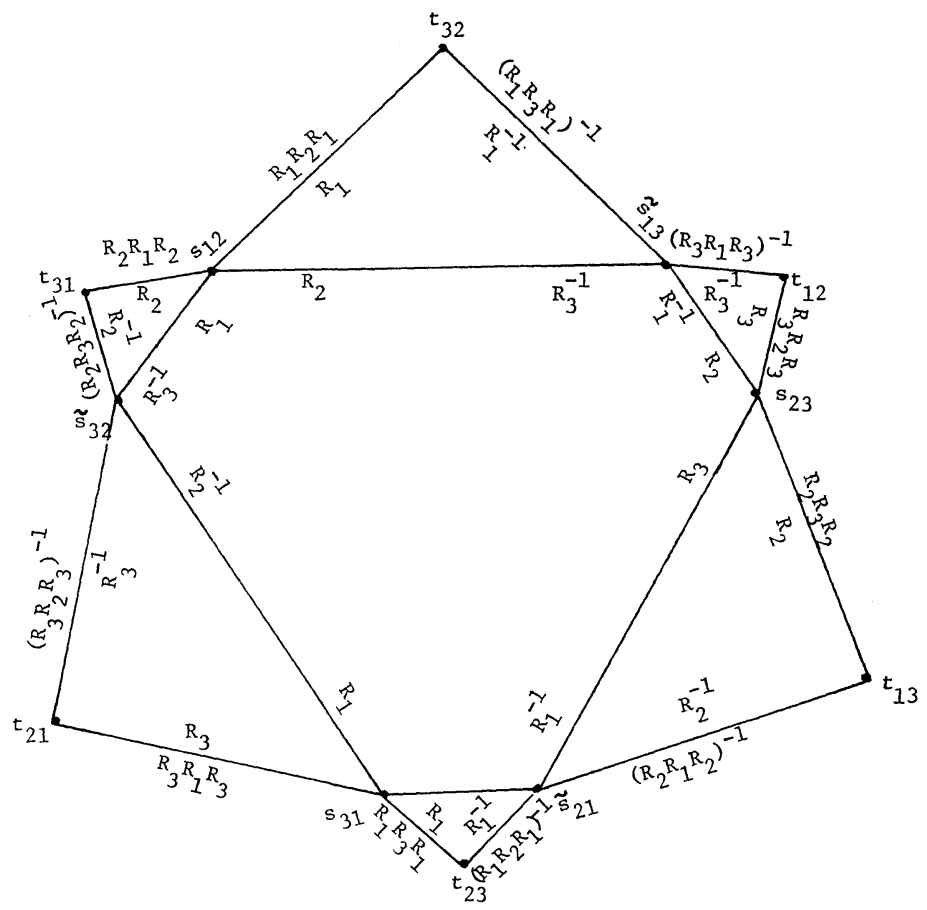

FIGURE 14.1. A schematic drawing of $\Delta_{i j k}=I_{i j k} \cap F(\varphi)$ for $\left|\arg \varphi^{3}\right|<\pi / 2-\pi / p$. As $\varphi^{3} \rightarrow \bar{\eta} i, \Delta_{321}$ approaches the point at $\infty$. For $\arg \varphi^{3}>\pi / 2-\pi / p, \Delta_{321}$ becomes a single (finite) point in the ball so long as $\arg \varphi^{3}<3(\pi / 2-\pi / p)$. For $-3(\pi / 2-\pi / p)<\arg \phi^{3} \leqq$ $-(\pi / 2-\pi / p), \Delta_{123}$ is a single point.

Lemma 14.2.2. Assume $\left\langle v_{i j k}, v_{i j k}\right\rangle>0$, then

(i) $R_{k} \hat{R}_{j} R_{k} \cap I_{i j k}=\hat{R}_{j} \cap I_{i j k}$

(ii) $\left(R_{i} \hat{R}_{j} R_{i}\right)^{-1} \cap I_{i j k}=\hat{R}_{j}^{-1} \cap I_{i j k}$

for all permutation $(i, j, k)$ of $(1,2,3)$. 
Proof. Set $l_{j}=\hat{R}_{j} \cap I_{i j k}$. Then for any $x_{1} \in l_{1}$, set $x_{2}=J x_{1}, x_{3}=$ $J^{2} x_{1}$. Then $d\left(x_{1}, p_{0}\right)=d\left(x_{2}, p_{0}\right)=d\left(x_{3}, p_{0}\right)$ since $J p_{0}=p_{0}$. By Lemma 13.3, $J=R_{i} R_{i+1}$ on $I_{i}$. Hence $d\left(R_{1} R_{2} R_{1} x_{1}, p_{0}\right)=d\left(R_{2} R_{1} R_{2} x_{1}, p_{0}\right)=$ $d\left(R_{2} x_{2}, p_{0}\right)=d\left(x_{2}, p_{0}\right)$ since $x_{2} \in \hat{R}_{2}$. Hence $d\left(R_{1} R_{2} R_{1} x_{1} p_{0}\right)=d\left(x_{1}, p_{0}\right)$. It follows that $l_{1} \subset R_{1} \hat{R}_{2} R_{1} \cap I_{312}$. By Lemma 3.2.7, $R_{1} \hat{R}_{2} R_{1} \cap I_{312}$ is a circular arc. Consequently $l_{1}=R_{1} \hat{R}_{2} R_{1} \cap I_{312}$. By symmetry $R_{j} \hat{R}_{k} R_{j} \cap$ $I_{i j k}=R_{j} \cap I_{i j k}$. Since $R_{j} R_{k} R_{j}=R_{k} R_{j} R_{k}$, (i) follows. (ii) follows from (i) by applying the automorphism $a_{i k}$.

Lemma 14.2.3. For all permutations (ijk) of (123) and for all $\varphi$ with $\left|\arg \varphi^{3}\right|<3(\pi / 2-\pi / p), R_{j} \hat{R}_{k} R_{j} \cap e_{j}^{\perp}$ is a geodesic line.

Proof. Assume first that $\left\langle v_{i j k}, v_{i j k}\right\rangle>0$. By Lemmas 14.2 and 14.1, $R_{j} \hat{R}_{k} R_{j} \cap I_{i j k}$ is a geodesic line $l_{j}$ which contains $t_{i k}$. By Lemma 3.2.7. (iv) $l_{j}$ and the spine of $R_{j} \hat{R}_{k} R_{j}$ span a geodesic $R$-2-plane $G$. The $C$-line $e_{j}^{\perp}$ is orthogonal to $I_{i j k}$ at $t_{i k}$. By Lemma 3.2.8, $R_{j} \hat{R}_{k} R_{j} \cap$ $e_{j}^{!}$is the geodesic line, for it coincides with $G \cap e_{j}^{\perp}$.

By the analytic continuation argument of Remark 1 following Lemma 14.2, $R_{j} \hat{R}_{k} R_{j} \cap e_{j}^{\perp}$ is a geodesic line for all $\varphi$ with $\left|\arg \varphi^{3}\right|<$ $3(\pi / 2-\pi / p)$.

LEMma 14.2.4. For all permutations (ijk) of (123) and for all $\varphi$ with $\left|\arg \varphi^{3}\right|<3(\pi / 2-\pi / p)$,

(i) $\hat{R}_{i}^{-1} \cap I_{i j k}$ is a geodesic line.

(ii) $\hat{R}_{i}^{-1} \cap I_{i j k}=\hat{R}_{k} \cap I_{i j k}$.

Proof. By Lemma 14.2.1(i)', $\hat{R}_{i}^{-1} \cap I_{i}^{\prime}$ is a geodesic line. By Remark 2 following Lemma 12.3, $I_{i}^{\prime}$ is orthogonal to $I_{i+1}$ at $\widetilde{s}_{i+1, i}$. By the argument above based on Lemma 3.2.8, $\hat{R}_{i}^{-1} \cap I_{i+1}$ is a geodesic line. By symmetry one infers that $\hat{R}_{i}^{-1} \cap I_{i j k}$ is a geodesic line as asserted in (i). By applying the isometry $a_{i k}: V(\bar{\varphi}) \rightarrow V(\varphi)$, one infers that $\hat{R}_{k} \cap I_{i j k}$ is a geodesic line. By Lemma 13.4, both $\hat{R}_{i}^{-1} \cap I_{i j k}$ and $\hat{R}_{k} \cap$ $I_{i j k}$ contain the points $s_{j k}$ and $\widetilde{s}_{j i}$. Since geodesic lines in $C h^{n}$ are unique, (ii) follows.

14.3. The region $\Omega(\varphi)$.

We have defined in $\S 10.2$

$$
F(\varphi)=F_{12} \cap F_{23} \cap F_{31} .
$$

From the results of $\S 10.1$, we have

$$
s_{i j} \in F_{12}, \widetilde{s}_{i j} \in F_{12}
$$

for all distinct $i, j$ (modulo 3). Also 


$$
t_{13}, t_{23}, t_{31}, t_{32} \in F_{12} \text {. }
$$

By symmetry it follows that for $i \neq j$,

$$
s_{i j}, \tilde{s}_{i j} \in F_{12} \cap F_{23} \cap F_{31}
$$

and also

$$
t_{i k}, t_{k i} \in F_{i j} \cap F_{j k}
$$

and as a matter of fact for all permutations of $i, j, k$, the points $p_{i j}, i_{i j}, \widetilde{s}_{i j}, t_{i k}$ are in $F(\varphi)$.

We would like to assert that the above points make up all the vertices of the cell $F(\varphi)$. To verify this, we would have to show that the faces of the region $F_{12}$ intersect the edges of $F(\varphi)$ in no points other than the above vertices. Proving this would entail estimates on the derivatives of $\left(\cosh d\left(x, p_{0}\right)\right)^{2}-\left(\cosh d\left(\gamma x, p_{0}\right)\right)^{2}$ and their behavior on the faces of $F(\varphi)$. We circumvent this difficulty in the following way.

Let $\Delta_{i j}=\left\{R_{i}^{ \pm 1},\left(R_{i} R_{j}\right)^{ \pm 1},\left(R_{i} R_{j} R_{i}\right)^{ \pm 1} ; i \neq j=1,2,3\right\}$, and let $\Delta=$ $\Delta_{12} \cup \Delta_{23} \cup \Delta_{31}$. As in $\S 10$ set $F_{12}=\bigcap_{r \in A_{12}} \gamma^{+}$. Let $E_{k}\left(F_{12}\right)$ denote the set of codimension $k$-faces of $F_{12}$. The set $E_{2}^{12}\left(F_{12}\right)$ consists of twenty 2-faces; set ${ }^{*} e_{i}^{\perp}={ }^{*} R_{i} \cap{ }^{*} R_{i}^{-1}$ (where ${ }^{*} \gamma=\hat{\gamma} \cap F_{12}$ for $\gamma \in \Delta_{12}$ ), $i=1,2$. Then for

(i) any $e \in E_{2}\left(F_{12}\right), e \neq{ }^{*} e_{1}^{\perp}$ or ${ }^{*} e_{2}^{\perp}$, the information of $\S 14.2$ shows that $e \cap F(\varphi)$ lies in a curvilinear triangle with vertex at $p_{12}$ and opposite edge a geodesic line.

(ii) $e_{1}^{\perp} \cap F(\varphi)$ lies in a geodesic quadrilateral $p_{12} t_{32} p_{31} t_{23}$ bounded the geodesic lines

$$
e_{1}^{\perp} \cap R_{1} \widehat{R_{2}} R_{1}, e_{1}^{\perp} \cap\left(\widehat{R_{2} R_{1}}\right)^{-1}, e_{1}^{\perp} \cap \widehat{R_{3} R_{1} R_{3}}, e_{1}^{\perp} \cap\left(\widehat{R_{2} R_{1}}\right)^{-1} .
$$

For each $e \in E_{2}\left(F_{12}\right)$, define the $\widetilde{e}$ as the above curvilinear triangle if $e \neq e_{i}^{\perp}$ and as the geodesic quadrilateral for $e=e_{1}^{\perp}$.

By use of the automorphism $J$, we define $\widetilde{e}$ for $e \in E_{2}\left(F_{12}\right) \cup$ $E_{2}\left(F_{23}\right) \cup E_{3}\left(F_{23}\right)$. (It is easy to verify that the resulting $\widetilde{e}_{2}^{\perp}=J^{*} e_{1}^{\perp}$ coincides with the geodesic quadrilateral obtainable from ${ }^{*} e_{2}^{\perp} \in E_{2}\left(F_{12}\right)$.) For any $\gamma \in \Delta$, we define $\tilde{\gamma}$ as the region of $\hat{\gamma}$ that is bounded by the 2-faces $\widetilde{e}$ lying in $\hat{\gamma}$. Finally, we define $\Omega(\varphi)$ as the 4-dimensional region which is bounded by $\{\widetilde{\gamma} ; \gamma \in \Delta\}$. It is clear that $F(\varphi) \subset \Omega(\phi)$. Computer calculations for certain values of $\varphi$ of interest to us, shows that in all such cases $F(\varphi)=\Omega(\varphi)$.

Indeed, for all cases in which $\Omega(\varphi)$ satisfies (CD1) and (CD2), $\Omega(\varphi)$ is a fundamental domain mod Aut $\Omega$ by Theorem 6.3.2. On the other hand, if $D=\bigcap_{r \in} \gamma^{+}$, then $\Gamma D=X$. Therefore $\Omega \subset$ (Aut $\left.\Omega\right) D \subset$ Aut $\Omega \cdot F=F$. Consequently $F(\varphi)=\Omega(\varphi)$ whenever $\Omega(\varphi)$ satisfies (CD1) and (CD2). 
Henceforth we focus attention on $\Omega(\varphi)$ rather than $F(\varphi)$. The 3-faces $\widetilde{R_{1}}, \widetilde{R_{1} R_{2}}, \widetilde{R_{1}} \widetilde{R_{2}} R_{1}$ in case $\left|\arg \varphi^{3}\right|<\pi / 2-\pi / p$ are shown below. The 3-faces $\widetilde{R}_{1}^{-1},\left(\widetilde{R_{2} \widetilde{R}_{1}}\right)^{-1},\left(\widetilde{R_{1} \widetilde{R_{2}} R_{1}}\right)^{-1}$ can be obtained from the complex conjugation map $V(\bar{\varphi}) \rightarrow V(\varphi)$, and the faces $\widetilde{R}_{2}, \widetilde{R_{2} R_{1}}$ can be obtained by applying to these the isometry $a_{12}: V(\varphi) \rightarrow V(\varphi)$. The remaining faces can be obtained from the symmetry $J$.

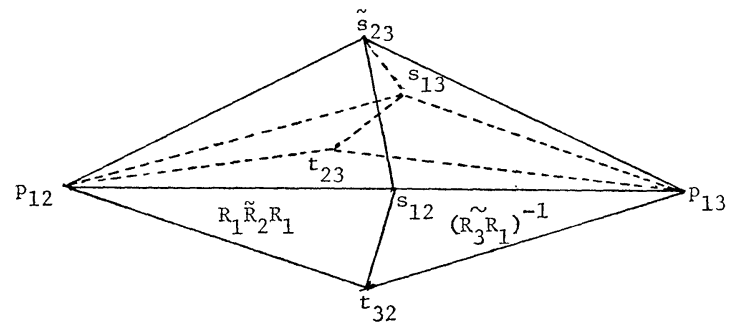

FIgURE 14.2. The nine faces of $\tilde{R}_{1}$ (labeled by the intersecting 3 -faces) for $\left|\arg \varphi^{3}\right|<\pi / 2-\pi / p$.

$R_{1} \widetilde{R_{2} R_{1}}\left(p_{12}, s_{12}, t_{32}\right), \widetilde{R_{2} R_{1}}\left(p_{12}, s_{12}, \widetilde{s_{32}}\right), \tilde{R}_{2}^{-1}\left(p_{12}, \bar{s}_{32}, s_{31}\right),\left(\widetilde{R_{2} R_{1}}\right)^{-1}\left(p_{12}, s_{31}, t_{23}\right)$ $\left(\widetilde{R_{3} R_{1}}\right)^{-1}\left(p_{31}, s_{12}, t_{32}\right), \tilde{R}_{3}^{-1}\left(p_{31}, s_{12}, \bar{s}_{32}\right), \widetilde{R_{3} R_{1}}\left(p_{31}, \bar{s}_{32}, s_{31}\right), \widetilde{R_{3} R_{1} R_{3}}\left(p_{31}, s_{31}, t_{23}\right)$ $\tilde{R}_{1}^{-1}\left(p_{12}, t_{32}, p_{31}, t_{23}\right)$

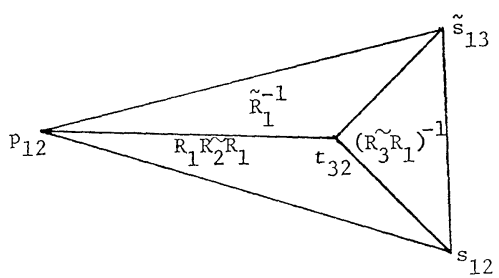

FiguRE 14.3. The four faces of $\widetilde{R_{1} R_{2}}$ for $-(\pi / 2-\pi / p)<\arg \varphi^{3}<3(\pi / 2-\pi / p)$. $R_{1} \tilde{R}_{2} R_{1}\left(p_{12}, s_{12}, t_{32}\right), R_{1}^{-1}\left(p_{12}, \widetilde{s_{31}}, t_{32}\right), \tilde{R}_{2}\left(p_{12}, s_{12}, \bar{s}_{13}\right),\left(\widetilde{R_{3} R_{1}}\right)^{-1}\left(t_{32}, s_{12}, \widetilde{s_{13}}\right)$

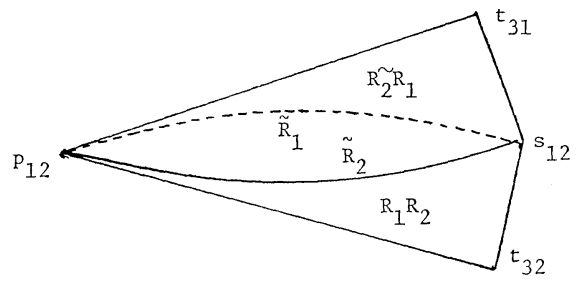

FIgURE 14.4. The four faces of $R_{1} \widetilde{R_{2} R_{3}}$ for $\left|\arg \varphi^{3}\right|<\pi / 2-\pi / p$. $\tilde{R}_{1}\left(p_{12}, s_{12}, t_{32}\right), \widetilde{R_{1} R_{2}}\left(p_{12}, s_{12}, t_{32}\right), R_{2}\left(p_{12}, s_{12}, t_{31}\right), \widetilde{R_{2} R_{1}}\left(p_{12}, s_{12}, t_{31}\right)$

14.4. Condition (CD1) for $\Omega(\varphi)$.

Proposition. For all $\varphi$ with $|\arg \varphi|<\pi / 2-\pi / p$, the region $\Omega(\varphi)$ satisfies condition (CD1). 
Proof. It suffices to prove that:

$$
\gamma: E_{k}(\tilde{\gamma})=E_{k}\left(\widetilde{\gamma}^{-1}\right)
$$

for $k=0,1,2$ and all $\gamma \in \Delta$. For this will imply that $\gamma$ maps the boundary of $\tilde{\gamma}$ onto the boundary of $\tilde{\gamma}^{-1}$ and therefore $\tilde{\gamma}$ onto $\tilde{\gamma}^{-1}$. Actually it suffices to prove (14.3.1) only for those 0,1,2-faces which do not contain the apex $p_{12}$. For by $\S 9$, each $F_{i j}$ is a fundamental domain for the finite group $\Gamma_{i j}$ and therefore satisfies (CD1) $i \neq j$, $i, j \in 1,2,3)$. The (CD1) condition for $F_{12}$, together with (14.3.1) for $e \in E_{k}(\widetilde{\gamma}) ; \gamma \in \Delta_{12}$, with $p_{12} \notin e, k=0,1,2$, yield $\gamma \cdot \tilde{\gamma}=\tilde{\gamma}^{-1}$ for all $\gamma \in \Delta_{12}$; by symmetry one gets

$$
\gamma \cdot \tilde{\gamma}=\tilde{\gamma}^{-1}, \text { all } \gamma \in \Delta .
$$

From the definition of $\Omega(\varphi)$, one sees that the only 2-faces of $\Omega(\varphi)$ which do not contain an apex are for any permutation (ijk) of $(1,2,3)$.

$$
\begin{array}{cl}
I_{i j k} & \text { for } \quad\left|\arg \varphi^{3}\right|<\frac{\pi}{2}-\frac{\pi}{p} \\
I_{123}, I_{231}, I_{312} & \text { for } \quad\left(\frac{\pi}{2}-\frac{\pi}{p}\right) \leqq \arg \varphi^{3}<3\left(\frac{\pi}{2}-\frac{\pi}{p}\right) \\
I_{321}, I_{132}, I_{213} & \text { for } \quad-3\left(\frac{\pi}{2}-\frac{\pi}{p}\right)<\arg \varphi^{3} \leqq-\left(\frac{\pi}{2}-\frac{\pi}{p}\right)
\end{array}
$$

and that each vertex and 1-face not containing an apex lies on the above 2 -faces.

Set

$$
\Delta_{i j k}=I_{i j k} \cap \Omega(\varphi) \text { for }\left\langle v_{i j k}, v_{i j k}\right\rangle>0 \text {. }
$$

From the results of $\S 14$ and $\S 10$, one sees that every $e \in E_{1}(\Omega)$ which lies in some $\tilde{\gamma} \in E_{1}(\Omega)$ but does not lie in any $f \in E_{2}\left(F_{12}\right)$ is a geodesic line segment. Inasmuch as the geodesic joining any two points in $C \mathrm{~h}^{n}$ is unique, to prove (CD1) for $\Omega$, it suffices to prove (14.3.1) for $k=0$; for reasons of symmetry, we need only consider $\gamma \in \Delta_{12}$, and $\gamma=R_{1}, R_{1} R_{2}$, or $R_{1} R_{2} R_{1}$. The requisite information

$$
\begin{aligned}
& R_{1} t_{32}=t_{32}, R_{1} s_{12}=\widetilde{s}_{13}, R_{1} \widetilde{s}_{32}=s_{23}, R_{1} s_{31}=\widetilde{s}_{21}, R_{1} t_{23}=t_{23} \\
& R_{1} R_{2} s_{12}=s_{23}, R_{1} R_{2} t_{32}=t_{13}, R_{1} R_{2} \widetilde{s}_{13}=\widetilde{s}_{21} \\
& R_{1} R_{2} R_{1} t_{32}=t_{13}, R_{1} R_{2} R_{1} s_{12}=\widetilde{s}_{21}, R_{1} R_{2} R_{1} t_{31}=t_{23}
\end{aligned}
$$

is given in $\S 13$.

15. $\left.\left(\widehat{R_{i} \hat{R}_{j}}\right)^{-1}, \widehat{R_{j} R_{k}}\right)$ and related angles. 
In order to determine for which values of the parameter $\varphi$ the region $\Omega(\phi)$ satisfies condition (CD2), we compute the angles at which the 3-faces of $\Omega(\varphi)$ meet at those $e \in E_{2}(\Omega)$ which do not contain an apex of $\Omega$. Such 2 -faces are $\Delta_{i j k}$ corresponding to all permutations (ijk) of (123) if $\left|\arg \varphi^{3}\right|<\pi / 2-\pi / p$, to the even permutations if $\pi / 2-\pi / p \leqq \arg \varphi^{3} \leqq \arg \varphi^{3} \leqq 3(\pi / 2-\pi / p)$ and to the odd permutations if $-3(\pi / 2-\pi / p)<\arg \varphi^{3} \leqq-\pi / 2-\pi / p$.

Lemma 15.1. (i) For any permutation (ijk) of $(1,2,3)\left(R_{i} \hat{R}_{j}\right)^{-1}$ and $\widehat{R}_{j} \widehat{R}_{k}$ meet in $I_{i j k}$ at a constant angle if $\left\langle v_{i j k}, v_{i j k}\right\rangle>0$. Moreover (ii) $\Varangle\left(\left(\widehat{R_{1} R_{2}}\right)^{-1}, \widehat{R_{2} R_{3}}\right)=\pi / 2-\pi / p+\arg \varphi^{3} \quad$ for $\quad \arg \varphi^{3} \geqq$ $-(\pi / 2-\pi / p)$

(iii) $\Varangle\left(\widehat{R_{3} R_{2}}\right)^{-1}, \widehat{R_{2} R_{1}}=\pi / 2-\pi / p-\arg \varphi^{3}$ for $\arg \varphi^{3} \leqq \pi / 2-\pi / p$.

Proof. By Lemma 11.1, $I_{i j k}$ is a common slice of $\left(\widehat{R_{i} R_{j}}\right)^{-1}$ and $\widehat{R_{j} R_{k}}$. By Lemma 3.2.9 (ii) the spines of $\left(\widehat{R_{i} R_{j}}\right)^{-1}$ and $\widehat{R_{j} R_{k}}$ lie in the same $C$-line. Hence by Lemma 3.2.4, (i) follows. The angle $\Varangle\left(\left(\widehat{R_{1} R_{2}}\right)^{-1}\right.$, $\left.\widehat{R_{2} R_{3}}\right)$ can be computed as $\Varangle\left(z\left(\left(\widehat{R_{1} R_{2}}\right)^{-1}\right), z\left(\widehat{R_{2} R_{3}}\right)\right)$ for any $C$-valued $S$-function $\left(S=\left(\widehat{R_{1} R_{2}}\right)^{-1}, \widehat{R_{2} R_{3}}\right)$ by Lemma 3.2.6. By (11.2), for such a function $z$, we get as the images of spinal surfaces

$$
\begin{aligned}
& \left(\widehat{R_{1} R_{2}}\right)^{-1}:\left|z+\eta^{2-2} \varphi\right|=1 \\
& R_{2} \hat{R}_{3}: \quad|z-\eta i \varphi|=1 .
\end{aligned}
$$

The angle $\theta$ at which the normals to the two circles at 0 intersect is clearly

$$
\begin{gathered}
\left(\eta i \varphi /-\eta^{2} \bar{\varphi}^{2}\right) \\
\theta=\arg \left(\eta i \varphi=\eta^{2-2} \varphi\right)=\arg \left(-\bar{\eta} i \varphi^{3}\right) .
\end{gathered}
$$

Hence the angle at which the two circles intersect is $|\pi-\theta|=$ $\pi / 2-\pi / p+\arg \varphi^{3}$ for $\arg \varphi^{3} \geqq-(\pi / 2-\pi / p)$. This proves (ii). (iii) is obtained from (ii) by applying the isometry $J^{\prime}: V(\bar{\varphi}) \rightarrow V(\varphi)$, wnich interchanges 1 and 3 .

LEMMA 15.2(i). In the geodesic triangle $\Delta_{\imath j k}$

$$
\ngtr t_{i k} s_{j k} \widetilde{s}_{j i}=\begin{aligned}
& \frac{1}{2}\left(\frac{\pi}{2}-\frac{\pi}{p}-\arg \varphi^{3}\right), \text { for even }(i j k) \\
& \frac{1}{2}\left(\frac{\pi}{2}-\frac{\pi}{p}+\arg \varphi^{3}\right), \text { for odd }(i j k)
\end{aligned}
$$

where $(i j k)$ is a permutation of (123).

Proof. By symmetry, it suffices to consider the geodesic triangle 
$\Delta_{312}$. The calculation is somewhat analagous to the one used in proving Lemma 15.1. We shall first determine the equations of the lines $\widetilde{R}_{1} \cap \Delta_{212}$ and $\widetilde{R}_{2} \cap \Delta_{312}$. From the matrices of $\S 9$, we get as the equations of $\hat{R}_{1}$ and $\hat{R}_{2}$

$$
\begin{aligned}
& \hat{R}_{1}:\left|\eta^{2} x_{1}+(1-\eta i \bar{\varphi}) x_{2}+(1-\eta i \varphi) x_{3}\right|=\left|x_{1}+x_{2}+x_{3}\right| \\
& \hat{R}_{2}:\left|(1-\eta i \varphi) x_{1}+\eta^{2} x_{2}+(1-\eta i \bar{\varphi}) x_{3}\right|=\left|x_{1}+x_{2}+x_{3}\right|
\end{aligned}
$$

From (11.3a) we get as the equation of $I_{312}$

$$
I_{312}: x_{3}=-\eta i \varphi x_{2} \text {. }
$$

Substituting for $x_{3}$ and setting $z=x_{2} /\left(x_{1}+x_{2}+x_{3}\right)$

$$
\begin{aligned}
& I_{312} \cap \hat{R}_{1}:\left|z(1-\eta i \bar{\varphi})\left(1-\eta i \varphi-\eta^{2} \varphi^{2}\right)+\eta^{2}\right|=1 \\
& I_{312} \cap \hat{R}_{2}:\left|z\left(-1+\eta i \varphi+\eta^{2} \varphi^{2}\right)+\eta^{2}\right|=1 .
\end{aligned}
$$

Setting $b=1-\eta i \varphi-\eta^{2} \varphi^{2}$, we get

$$
\begin{aligned}
& I_{312} \cap \hat{R}_{2}:\left|z+\eta^{2} / b(1-\eta i \bar{\varphi})\right|=1 /|b(1-\eta i \bar{\varphi})| \\
& I_{312} \cap \hat{R}_{1}:|z-(1-\eta i \varphi) / b|=1 /|b| .
\end{aligned}
$$

The common solution corresponding to the point $s_{12}=\bar{\eta} i \varphi e_{1}+\bar{\eta} i \bar{\varphi} e_{2}+e_{3}$ is

$$
s_{12}: z=\bar{\eta} i \bar{\varphi} /(1+\bar{\eta} i(\varphi+\bar{\varphi})) .
$$

The map of the $C$-line $I_{312}$ into $C$ given by the function $z$ is holomorphic and therefore conformal. It follows from elementary geometry of circles that one of the two angles between the two circles is the arc of

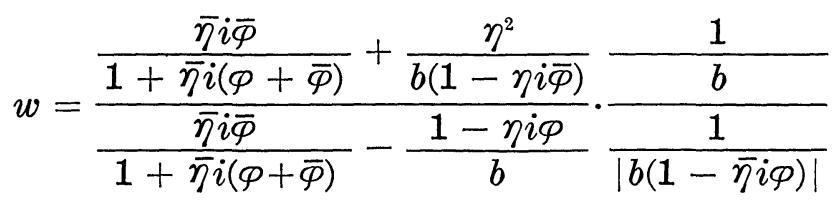

After clearing denominators and simplifying numerators we get

$$
w=\frac{\left(1+\bar{\eta} i \bar{\varphi}+\bar{\eta}^{2}\right)(1-\eta i \bar{\varphi})}{\left(1+\bar{\eta} i \varphi+\eta^{2}\right)(1-\eta i \bar{\varphi})}
$$

Note that $1+\eta i \bar{\varphi}+\bar{\varphi}^{2}=\bar{\varphi}^{2}\left(1+\eta i \varphi+\eta^{2}\right)$. Hence

$$
\begin{aligned}
w^{2} & =\varphi^{-4} \frac{(1-\eta i \bar{\varphi})(1+\bar{\eta} i \bar{\varphi})}{(1-\eta i \bar{\varphi})(1-\eta i \bar{\varphi})} \\
& =\varphi^{-4} \frac{\bar{\eta} i \varphi(1-\eta i \bar{\varphi})}{1-\eta i \bar{\varphi}} \\
& =\bar{\eta} i \bar{\varphi}^{3} .
\end{aligned}
$$


It follows that $\Varangle t_{32} s_{12} \widetilde{s}_{13}=\left(\pi / 2-\pi / p-\arg \varphi^{3}\right) / 2$. From this the lemma follows.

REMARK 1. The angles above can be computed by applying the analogue of Napier's rule for a right triangle $A B C$ with hypotenuse $c$ :

$$
\cot A \cot B=\cosh 2 c
$$

in the constant curvature $C$-line $I_{i j k}$. The resulting computation is longer than the one presented here.

REMARK 2. Note that the triangle $\Delta_{i j k}$ is isosceles since $R_{j}$ carries one side into another by Lemmas 14.2.1(i) and 13.4. Hence

$$
\Varangle t_{i k} s_{j k} \widetilde{s}_{j i}=\Varangle t_{i k} \widetilde{s}_{j l} s_{j k}=\frac{1}{2} \Varangle\left(\left(\widehat{R_{k} R_{j}}\right)^{-1}, \widehat{R_{j} R_{i}}\right)
$$

for $\left|\arg \varphi^{3}\right|<\pi / 2-\pi / p$.

LeMma 15.3. $\Varangle t_{13} p_{12} t_{31}=((6-p) / 2 p) \pi(p=3,4,5)$.

Proof. Let $\Delta_{i}=F_{12} \cap e_{i}^{\perp}(i=1,2)$. From the description of the fundamental domain $F_{12}$ in $\S 10.1$, we see that $F_{12}$ has only two 2dimensional faces fixed under a $C$-reflection; namely $\Delta_{1}$ and $A_{2}$. Consequently, from $X=\Gamma_{12} F_{12}$ we infer

$$
e_{1}^{\perp}=\bigcup\left\{\gamma \Delta_{2} ; \gamma \Delta_{2} \subset e_{2}^{\perp}\right\} \cup\left\{\gamma \Delta_{1} ; \gamma \Delta_{1} \subset e_{2}^{\perp}\right\} .
$$

Clearly $\gamma \Delta_{1} \subset e_{2}^{\perp}$ implies $\gamma e_{2}^{\perp}=e_{2}^{\perp}$ and therefore $\gamma \in Z \Gamma_{2}$ where $Z$ is the center of $\Gamma_{12}$ and $\Gamma_{2}=\left\{\left\{R_{2}\right\}\right\}$. We have $R_{1} R_{2} e_{1}=-\eta i \varphi e_{2}$ by $\S 9.1$. Hence $R_{1} R_{2} R_{1} e_{1}^{\perp}=R_{1} R_{2} e_{1}^{\perp}=e_{2}^{\perp}$. Inasmuch as $Z e_{i}^{\perp}=e_{i}^{\perp}(i=1,2)$ and $\left(R_{1} R_{2} R_{1}\right)^{2} \in Z$, we see that

$$
\left\{\gamma \in \Gamma_{12} ; \gamma \Delta_{2} \subset e_{1}^{\perp}\right\}=Z R_{1} R_{2} R_{1} \Gamma_{2} .
$$

Hence

$$
e_{2}^{\perp}=Z \Delta_{2} \cup Z R_{1} R_{2} R_{1} \Delta_{1} .
$$

Thus $e_{2}^{\perp}$ is a union of $2 \# Z$ sectors with disjoint interiors. It follows at once that

$$
\Varangle t_{13} p_{12} t_{31}=\frac{1}{2 \# Z} 2 \pi=\frac{6-p}{2 p} \pi
$$

by (2.2.4).

16. The stabilizer of $\Omega(\varphi)$ in $\Gamma$.

Let $\rho$ be order (in the multiplicative group of nonzero complex 
numbers) of $\bar{\eta} i \varphi^{3}$, and let $\sigma$ be the order of $\bar{\eta} i \bar{\varphi}^{3}$. Let

$$
\begin{aligned}
& r=\operatorname{order}\left(R_{1} R_{2} R_{3}\right)^{2} \quad \text { in } \mathrm{PU}(H) \\
& s=\operatorname{order}\left(R_{3} R_{2} R_{1}\right)^{2} \quad \text { in } \mathrm{P}(\mathrm{U})(H) .
\end{aligned}
$$

Let $\operatorname{Aut}_{\Gamma} \Omega$ denote the stabilizer in $\Gamma$ of the region $\Omega(\varphi)$. From $\S 9.2$, we know that the order $\# \operatorname{Aut}_{\Gamma} \Omega$ is at most 3 . The following theorem determines Aut $_{\Gamma} \Omega$.

Lemma 16.1. Assume that $\rho$ is finite (or equivalently that $\sigma$ is finite) $\bmod \rho$

(i) if $3 \nmid \rho$ then $\left(R_{1} R_{2} R_{3}\right)^{2 \mu} R_{1} R_{2}=J$ in $C h^{2}$, where $3 \mu+1 \equiv 0$

(ii) if $3 \nmid \sigma$, then $\left(R_{3} R_{2} R_{1}\right)^{2 \nu} R_{3} R_{2}=J^{-1}$ in $C h^{2}$, where $3 \nu+1 \equiv 0$ $\bmod \sigma$,

(iii) $\quad\left(R_{1} R_{2} R_{3}\right)^{2 \mu} R_{1} R_{2}=\left(R_{2} R_{3} R_{1}\right)^{2 \mu} R_{2} R_{3}=\left(R_{3} R_{1} R_{2}\right)^{2 \mu} R_{3} R_{1}$ $\left(R_{3} R_{2} R_{1}\right)^{2 \nu} R_{3} R_{2}=\left(R_{1} R_{3} R_{2}\right)^{2 \nu} R_{1} R_{3}=\left(R_{2} R_{1} R_{3}\right)^{2 \nu} R_{2} R_{1}$

(iv) $r=\operatorname{order}\left(\bar{\eta} i \varphi^{3}\right)^{3}, s=\operatorname{order}\left(\bar{\eta} i \bar{\varphi}^{2}\right)^{3}$.

Proof. Set $\xi=\bar{\eta} i \varphi^{3}$. From (9.1.1) we see that the eigenvalues $\left(R_{1} R_{2} R_{3}\right)^{2}$ are

$$
-\eta^{6} \varphi^{-6}, \quad \eta^{3} i \varphi^{3}, \quad \eta^{3} i \varphi^{3}
$$

In addition by (9.1.3)

$$
\begin{aligned}
& \left(R_{1} R_{2} R_{3}\right)^{2} v_{123}=-\eta^{6} \Phi^{-6} v_{123} \\
& \left(R_{1} R_{2} R_{3}\right)^{2} e_{2}=\eta^{3} i \varphi^{3} \\
& \left\langle e_{2}, v_{123}\right\rangle=0 .
\end{aligned}
$$

This implies that $\left(R_{1} R_{2} R_{3}\right)^{2}$ fixes the point $\pi\left(v_{123}\right)$ of $C P^{2}$, and each point of the complex projective line $\pi\left(v_{123}^{\perp}\right)$, rotates $C P^{2}$ around the point $\pi\left(v_{123}\right)$ by the scalar multiple

$$
\eta^{3} i \varphi^{3} /-\eta^{6} \varphi^{-6}=\bar{\xi}^{3}
$$

stabilizes $e_{2}^{\perp}$, and rotates the complex projective line $\pi\left(e_{2}^{1}\right)$ around the points

$$
\pi\left(v_{123} \cap e_{2}^{\perp}\right) \text { and } \pi\left(v_{123}^{\perp} \cap e_{2}^{\perp}\right) \quad \text { by }
$$

scalar multiples $\left(\eta i \varphi^{3}\right) /\left(-\eta^{6} \varphi^{-6}\right)=\bar{\xi}^{3}$ and $\xi^{3}$ respectively.

The order of $\left(R_{1} R_{2} R_{3}\right)^{2}$ in $\mathrm{PU}(H)$ is the order of $\xi^{3}$. This proves the first part of (iv) and proof of the second part is similar.

By Lemma 12.3

$$
t_{13}=\left\{\begin{array}{lll}
\pi\left(v_{123}^{\perp} \cap e_{2}^{\perp}\right) & \text { if }\left\langle v_{123}, v_{123}\right\rangle>0 \\
\pi\left(v_{123} \cap e_{2}^{\perp}\right) & \text { if }\left\langle v_{123}, v_{123}\right\rangle<0
\end{array}\right.
$$


From $\S 9.1$ we see that $R_{3} R_{1} e_{3}=-\eta i \varphi e_{1}$ (by symmetry from $\left.R_{1} R_{2} e_{1}=-\eta i \varphi e_{2}\right)$ so that $R_{3} R_{1} e_{3}^{\perp}=e_{1}^{\perp}$. By Lemma 13.5, $R_{3} R_{1} t_{21}=t_{32}$. Hence $R_{3} R_{1}$ sends the quadrilateral $\boldsymbol{Q}_{3}=\left[p_{31} t_{12} p_{23} t_{21}\right]$ into the $\boldsymbol{C}$-line $\pi\left(e_{1}^{\perp}\right)$ so as to abot the quadrilateral $\boldsymbol{Q}_{1}=\left[p_{12} t_{23} p_{31} t_{32}\right]$ along the geo-

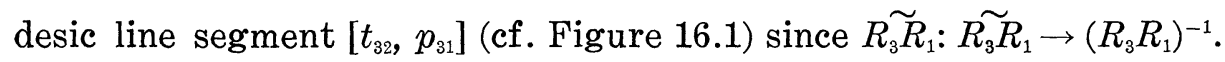

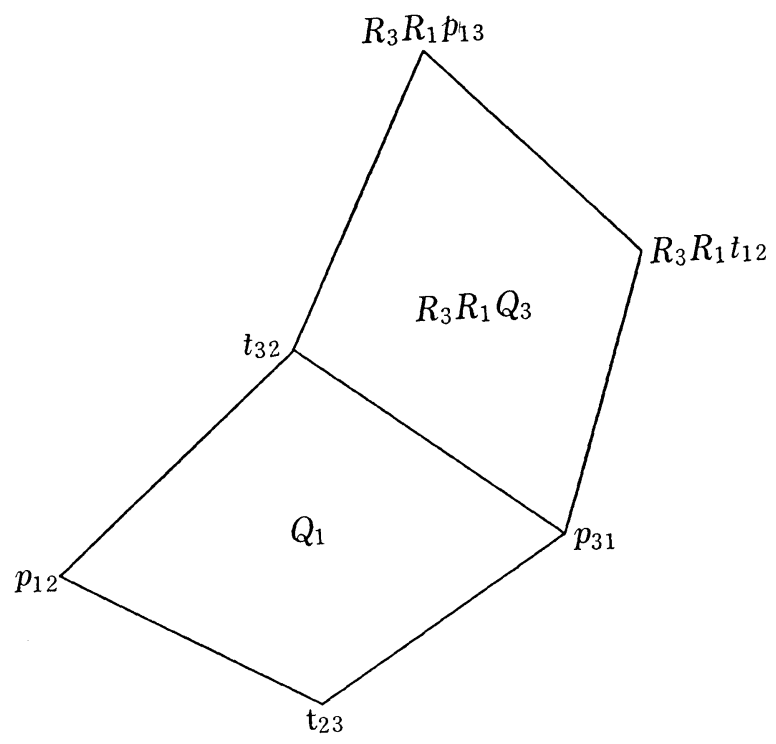

FIGURE 16.1

By symmetry one sees that image $R_{3} R_{1} \cdot R_{2} R_{3} \cdot R_{1} R_{2} Q_{1}$ is given by rotation about $t_{32}$ through the angle $3 \Varangle\left(R_{3} R_{1}\right)^{-1}, R_{1} R_{2}=3(\pi / 2-\pi / p+$ $\left.\arg \varphi^{3}\right)=3 \arg \xi$ in the sense from $p_{12}$ to $p_{31}$, this is a rotation about the point $t_{32}$ by the scalar multiple $\xi^{3}$ if $\left\langle v_{123}, v_{123}\right\rangle>0$ and by the scalar multiple $\bar{\xi}^{3}$ if $\left\langle v_{123}, v_{123}\right\rangle<0$.

Suppose now that $3 \nmid \rho$. Then one can choose $\mu$ so that $\left(R_{1} R_{2} R_{3}\right)^{2 \mu}$ rotates $R_{3} R_{1} Q_{3}$ back into $Q_{1}$; one need only select $\mu$ so that

$$
\begin{aligned}
& \left(\xi^{3}\right)^{\mu}=\bar{\xi}^{1} \quad \text { if } \quad\left\langle v_{123}, v_{123}\right\rangle>0 \\
& \left(\bar{\xi}^{3}\right)^{\mu}=\bar{\xi}^{-1} \quad \text { if } \quad\left\langle v_{123}, v_{123}\right\rangle<0 .
\end{aligned}
$$

In either case $\xi^{3 \mu+1}=1$; so select $\mu$ to satisfy $3 \mu+1 \equiv 0(\bmod \rho)$. Then we have

$$
\begin{aligned}
& \left(R_{3} R_{1} R_{2}\right)^{2 \mu} R_{3} R_{1} R_{31}=p_{12} \\
& \left(R_{3} R_{1} R_{2}\right)^{2 \mu} R_{2} R_{1} p_{23}=p_{31} .
\end{aligned}
$$

In addition, by Lemma 13.3 (i), $R_{3} R_{1}=J$ on $I_{3}$ sending $I_{3}$ to $I_{1}$ if $\left\langle v_{123}, v_{123}\right\rangle>0$. In this case, $\left(R_{3} R_{1} R_{2}\right)^{2 \mu} R_{3} R_{1}=J$ on $I_{3}$ (by Lemma 13.3(ii)) as well as on $\pi\left(e_{3}^{\perp}\right)$ and therefore on the ball.

It remains to consider the case $\left\langle v_{123}, v_{123}\right\rangle<0$. In this case, the 
triangles $\Delta_{312}, \Delta_{123}, \Delta_{231}$ collapse to points and the reasoning of the proof of Lemma 13.3(i) does not apply. Instead, one argues as follows: $R_{3} R_{1}$ carries $I_{3}$ to $I_{1}$ and $R_{3} R_{1} \cdot R_{2} R_{3} \cdot R_{1} R_{2}$ carries $I_{1}$ to $I_{1}$ rotating the $\boldsymbol{C}$-line $I_{1}$ about the point $t_{32}$ by the scalar factor $\bar{\xi}^{3}$. Hence by symmetry $R_{3} R_{1}$ restricted to $I_{3}$ is the map $\rho_{1} \circ J$ where $\rho_{1}$ is the rotation of the ball about $t_{32}$ by the scalar multiple $\bar{\xi}$. Consequently, $R_{3} R_{1}=\rho_{1} \cdot J$. By (16.1), $\left(R_{3} R_{1} R_{2}\right)^{2 \prime \prime}=\rho_{1}^{-1}$. Consequently $J=\rho_{1}^{-1} R_{3} R_{1}=\left(R_{3} R_{1} R_{2}\right)^{2 \mu} R_{3} R_{1}$. This proves (i).

(ii) follows from (i) by applying $J^{\prime}: V(\bar{\varphi}) \rightarrow(\varphi)$.

(iii) follows from (i) and (ii) by symmetry. The lemma is now proved.

REMARK 1. From (9.1.6) it follows that the order in $\mathrm{PU}(H)$ of $R_{2} R_{1} R_{2} R_{3}$ is 1.c.m.(order $\left.-\eta i \varphi^{3},-\eta i \bar{\rho}^{3}\right)=\operatorname{lcm}(\sigma, \rho)$.

REMARK 2. If $\Omega(\varphi)$ satisfies conditions (CD1) and (CD2), then $3 \mid \rho$ and $3 \mid \sigma$ implies that $\# \operatorname{Aut}_{\Gamma} \Omega=1$. For then the circuits around $\Delta_{i j l}$ result in the identity map. By Theorem 6.3.3(a), $\operatorname{Aut}_{\Gamma} \Omega=(1)$.

REMARK 3. More generally, in the joined $\Gamma \Omega(\varphi)$ space, if $3 \mid \rho$ and $3 \mid \sigma$, then $\# \operatorname{Aut}_{\Gamma} \Omega=1$, provided that $\left|\arg \varphi^{3}\right|<\pi / 2-\pi / p$. We shall prove this in $\S 18.4$.

Remark 4. By Lemma 13.3(i), $J^{-1} R_{1} R_{2}$ fixes each point of $I_{1}$. Clearly $J^{-1} R_{1} R_{2} p_{12}=J^{-1} p_{12}=p_{31}$. Consequently, $J^{-1} R_{1} R_{2}$ is a rotation of $C P^{2}$ about the line $I_{1}$ stabilizing the line $e_{1}^{\perp}$ and rotating $p_{12}$ into $p_{31}$ about $t_{32}$. Comparison with (16.1) shows that $J^{-1} R_{1} R_{2}$ is a rotation of $C P^{2}$ about $I_{1}$ by the multiple $\xi$. Moreover, the matrices $J^{-1} R_{1} R_{2}$ and $R_{1}$ commute. For $J R_{i}=R_{i+1} J$ for any $i \bmod 3$; hence

$$
J^{-1} R_{1} R_{2} R_{1}=J^{-1} R_{2} R_{1} R_{2}=R_{1} J^{-1} R_{1} R_{2} .
$$

17. Nonarithmetic lattices $\Gamma(\varphi)$.

17.1. Values of $\varphi$ for which $\Omega(\varphi)$ satisfies (CD2).

Set

$$
\begin{aligned}
& \rho=\operatorname{order}\left(\bar{\eta} i \varphi^{3}\right), \quad \sigma=\operatorname{order}\left(\bar{\eta} i \bar{\varphi}^{3}\right) \\
& r=\operatorname{order}\left(\bar{\eta} i \varphi^{3}\right)^{3}, \quad s=\operatorname{order}\left(\bar{\eta} i \bar{\varphi}^{3}\right)^{3} .
\end{aligned}
$$

From Lemma 16.1 we know that $r=\operatorname{order}\left(R_{1} R_{2} R_{3}\right)^{2}$ in $\mathrm{PU}(H)$, and $s=$ order $\left(R_{3} R_{2} R_{1}\right)^{2}$ in $\mathrm{PU}(H)$. From the fact that $F_{i j}$ is a fundamental domain for the finite group $\Gamma_{i j}$, we know that the codimension two condition (CD2) is satisfied for all 2-faces of $\Omega(\varphi)$ which contain an apex $p_{i j}, i \neq j, i, j \in\{1,2,3\}$. The only 2 -faces not containing an 
apex are $\Delta_{i j k}$ with $i j k$ ranging over all even permutations of (123) if $-(\pi / 2-\pi / p)<\arg \varphi^{3}<3(\pi / 2-\pi / p)$, and over all odd permutations if $-3(\pi / 2-\pi / p)<\arg \varphi^{3}<\pi / 2-\pi / p$. The circuit around $\Delta_{i j k}$ is $R_{i} R_{j} \cdot R_{k} R_{i} \cdot R_{j} R_{k} \cdots \Omega$. Each such circuit places side by side around $\Delta_{i j k}$ the region bounded by two spinal surfaces whose spines lie in a common $C$-line and which intersect in the slice $I_{i j k}$. By Lemma 15.1, $\left(\widehat{R_{i} R_{j}}\right)^{-1}$ and $\widehat{R_{j} R_{k c}}$ meet at a constant angle equal to $\pi / 2-\pi / p \pm$ $\arg \varphi^{3}$ (the $(+)$ or $(-)$ corresponding to even or odd permutations). Since $\left(\bar{\eta} i \varphi^{3}\right)^{\rho}=1$, the image of $\Omega(\varphi)$ after $\rho$ terms of the circuit coincides with $\Omega(\varphi)$, and by definition of $\rho$ no shorter circuit brings $\Omega(\varphi)$ back to coincidence with itself. On the other hand, by (CD2) all the interiors of the $\rho$ images of $\Omega(\varphi)$ in the circuit must be disjoint. Hence for $\left|\arg \varphi^{3}\right|<3(\pi / 2-\pi / p)$.

(i) $\rho\left(\pi / 2-\pi / p+\arg \varphi^{3}\right)=2 \pi$, if $\arg \varphi^{3}>-(\pi / 2-\pi / p)$.

Similarly,

(ii) $\sigma\left(\pi / 2-\pi / p-\arg \varphi^{3}\right)=2 \pi$, if $\arg \varphi^{3}<\pi / 2-\pi / p$. If $\left|\arg \varphi^{3}\right| \leqq \pi / 2-\pi / p$, both (i) and (ii) apply and adding we get

$$
\frac{1}{2}-\frac{1}{\rho}=\frac{1}{\rho}+\frac{1}{\sigma} \text {. }
$$

Setting $m=\inf (\rho, \sigma)$, this implies $m \leqq 4 p /(p-2) \leqq 2 m$. Thus

$$
\begin{aligned}
& \text { (iii) } 6 \leqq m \leqq 12 \text { if } p=3 \\
& 4 \leqq m \leqq 8 \text { if } p=4 \\
& 4 \leqq m \leqq 6 \quad \text { if } \quad p=5 \text {. }
\end{aligned}
$$

If $3(\pi / 2-\pi / p)>\arg \varphi^{3}>\pi / 2-\pi / p$, we see from (i) that

$$
\rho \cdot\left(\frac{1}{2}-\frac{1}{p}\right)<2<\rho \cdot 4\left(\frac{1}{2}-\frac{1}{p}\right) .
$$

Thus $p /(p-2)<\rho<2 p / p-2$, yielding

$$
\text { (iv) } \begin{aligned}
& 4 \leqq \rho \leqq 5 \text { for } p=3 \\
& 3 \leqq \rho \leqq 3 \text { for } p=4 \\
& 2 \leqq \rho \leqq 3 \text { for } p=5 .
\end{aligned}
$$

In this latter range for $\varphi, \Delta_{123} \Delta_{231}$, and $\Delta_{312}$ are the only 2-faces of $\Omega(\varnothing)$ not containing an apex.

In order to determine all the $\varphi$ for which $\Gamma(\varphi)$ is a discrete group it suffices to consider the range $0 \leqq \arg \phi^{3}<3(\pi / 2-\pi / p)$, inasmuch as the negative values of $\varphi$ are given by the symmetry $J^{\prime}: V(\varphi) \rightarrow V(\bar{\varphi})$. We shall give the $\varphi$ in two lists, one for $0 \leqq \arg \varphi^{3} \leqq$ $\pi / 2-\pi / p$, the other for $\pi / 2-\pi / p<\arg \varphi^{3}<3(\pi / 2-\pi / p)$. In case $\arg \varphi^{3}=\pi / 2-\pi / p$, the geodesic triangle $\Delta_{321}$ degenerates to a point 
at $\infty$ and it can be regarded as limiting value of $\arg \varphi^{3}>$ or $<$ $\pi / 2-\pi / p$; we list this case on our first table.

Set $t=(1 / \pi) \arg \phi^{3}$, then $t=2 / \rho-(1 / 2-1 / p)$. If $0 \leqq t$ then $\inf (\rho, \sigma)=\rho$. We therefore arrange the tables according to increasing values of the integer $\rho$. In Table $1,1 / \rho+1 / \sigma=1 / 2-1 / p$; in Table 2 , we have instead, $1 / \rho+1 / \sigma=t$. The requirement that $\sigma$ be an integer reduces the number

TABLE $1 . \quad 0 \leqq t \leqq 1 / 2-1 / p$

\begin{tabular}{rrrrrrr}
\hline$p$ & $\rho$ & $\sigma$ & \multicolumn{1}{c}{$t$} & \# Aut $_{\Gamma} \Omega$ & $r$ & \multicolumn{1}{c}{$s$} \\
\hline 3 & 6 & $\infty$ & $1 / 6$ & 1 & 2 & $\infty$ \\
3 & 7 & 42 & $5 / 42$ & 3 & 7 & 14 \\
3 & 8 & 24 & $1 / 12$ & 3 & 8 & 8 \\
3 & 9 & 18 & $1 / 18$ & 1 & 3 & 6 \\
3 & 10 & 15 & $1 / 30$ & 3 & 10 & 5 \\
3 & 12 & 12 & 0 & 1 & 4 & 4 \\
4 & 4 & $\infty$ & $1 / 4$ & 3 & 4 & $\infty$ \\
4 & 5 & 20 & $3 / 20$ & 3 & 5 & 20 \\
4 & 6 & 12 & $1 / 12$ & 1 & 2 & 4 \\
4 & 8 & 8 & 0 & 3 & 8 & 8 \\
5 & 4 & 20 & $1 / 5$ & 3 & 4 & 20 \\
5 & 5 & 10 & $1 / 10$ & 3 & 5 & 10 \\
\hline
\end{tabular}

TABLE 2. $1 / 2-1 / p<t<3(1 / 2-1 / p)$

\begin{tabular}{rrrrrrr}
\hline$p$ & $\rho$ & $\sigma$ & $t$ & \# Aut $_{\Gamma} \Omega$ & $r$ & \multicolumn{1}{c}{$s$} \\
\hline 3 & 4 & 12 & $1 / 3$ & 3 & 4 & 4 \\
3 & 5 & 30 & $7 / 30$ & 3 & 5 & 10 \\
4 & 3 & 12 & $5 / 12$ & 1 & 1 & 4 \\
5 & 2 & 5 & $7 / 10$ & 3 & 2 & 5 \\
5 & 3 & 30 & $11 / 30$ & 1 & 1 & 10 \\
\hline
\end{tabular}

of cases in (iii).

REMARK. Let $p=5, \varphi^{3}=i, \psi^{3}=\exp (7 \pi i / 10)$. We will see in $\S 21$ that $\Gamma(\varphi)$ is an arithmetic lattice despite the fact $\Omega(\varphi)$ does not satisfy (CD2). In this case $\Omega(\varphi)$ is not a fundamental domain $\bmod \mathrm{Aut}_{\Gamma} \Omega$; nevertheless, $\Gamma(\varphi)$ is a lattice. In fact, as we show in $\S 21$, there is an isomorphism of $\Gamma(\varphi)$ onto $\Gamma(\psi)$. Thus (CD2) is a sufficient condition for $\Gamma(\varphi)$ to be discrete but is not necessary. On the other hand, the failure of (CD2) for $\Omega(\varphi),\left|\arg \varphi^{3}\right|<\pi / 2-\pi / p$, seems to imply the $\Gamma(\phi)$ is not discrete. For example, (CD2) fails for $p=5$, $t=0$. In this case, one can compute that $\left.\left\langle R_{1} R_{2} R_{3}\right)^{9} e_{1}, e_{1}\right\rangle$ is a nonadmissible value (cf 2.4.3). This implies that $\left.\left\{R_{1} R_{2} R_{3}\right)^{9} R_{1}\left(R_{1} R_{2} R_{3}\right)^{-9}, R_{1}\right\}$ is a $C$-reflection group which fixes a point in the ball but is infinite. It follows that $\Gamma(1)$ is not discrete.

17.2. The field $\boldsymbol{Q}[\operatorname{Tr} \operatorname{Ad} \Gamma]$. In applying the test of $\S 4$ for 
arithmeticity of the lattice $\Gamma(\varphi)$, we must determine the field generated by $\operatorname{Tr} \operatorname{Ad} \gamma$, as $\gamma$ ranges over $\Gamma(\varphi)$; we denote this by $\boldsymbol{Q}[\operatorname{Tr} \operatorname{Ad} \Gamma]$. For any subfield $k$ of $C$ stable under complex conjugation, we denote by $\operatorname{Re} k$ the subfield $k \cap \boldsymbol{R}$. We continue the notation $\rho=$ order $\bar{\eta} i \varphi^{3}, \eta=e^{\pi i / p}$. For $\arg \varphi^{3}>-(\pi / 2-\pi / p), \bar{\eta} i \varphi^{3}=e^{2 \pi i / \rho /}$ by 17.1(i).

Lemma 17.2.1. $\boldsymbol{Q}[\operatorname{Tr} \operatorname{Ad} \Gamma]=\operatorname{Re} \boldsymbol{Q}\left[e^{2 \pi i / \rho}, 2 e_{z}{ }^{\pi i / p}\right]$ for $\arg \varphi^{3}>$ $-(\pi / 2-\pi / p)$ and for $\Gamma(\varphi)$ discrete.

Proof. We identify $\operatorname{Hom}(V, V)$ with $V \otimes V^{*}$ in the standard way so that for any $e, f \in V, \alpha, \beta \in V^{*}$,

$$
\begin{aligned}
& (e \otimes \alpha)(f)=\alpha(f) e \\
& (e \otimes \alpha)(f \otimes \beta)=\alpha(f) e \otimes \beta .
\end{aligned}
$$

Let $\left\{e^{1}, e^{2}, e^{3}\right\}$ denote the dual base to the base $\left\{e_{1}, e_{2}, e_{3}\right\}$ of our vector space $V(\varphi)$. We rewrite the generating $C$-reflections $R_{1}$ of $\Gamma(\varphi)$ :

$$
\begin{aligned}
& R_{1}(x)=x+\left(\eta^{2}-1\right)\left\langle x_{1} e_{1}\right\rangle e_{1}=\eta^{2} x_{1} e_{1}-\eta i \bar{\varphi} x_{2} e_{1}-\eta i \varphi x_{3} e_{1} \\
& R_{1}=\eta^{2} e_{1} \otimes e^{1}-\eta i \bar{\varphi} e_{1} \otimes e^{2}-\eta i \varphi e_{1} \otimes e^{3}+e_{2} \otimes e^{2}+e_{3} \otimes e^{3}
\end{aligned}
$$

and

$$
R_{j}=\sum_{k \neq j} e_{k} \otimes e^{k}+\sum_{k=1}^{3} a_{j k} e_{j} \otimes e^{k}, a_{i j}=\left\{\begin{array}{cc}
-\eta i \varphi^{\varepsilon(j, k)}, & j \neq k \\
\eta^{2} & , \quad j=k
\end{array}\right.
$$

where $\varepsilon(j, k)=(-1)^{k-j},(j, k=1,2,3)$. Then

$$
\begin{aligned}
\operatorname{Tr} R_{j_{1}} R_{j_{2}} \cdots R_{j_{l}} & =\sum_{q=1}^{l} \sum_{1 \leqq i_{1}<i_{2}<\cdots<i_{q}<l} a_{j_{i_{1}} j_{i_{2}}} a_{j_{i} j_{2} i_{3}} \cdots a_{j_{i_{q} i_{1}}} \\
& =\sum_{q}\left(\eta^{2}\right)^{q-m}(\eta i)^{m}\left(\varphi^{3}\right)^{n} \\
& =\sum_{q}\left(\eta^{2}\right)^{q}(\bar{\eta} i)^{m} \phi^{3 n}
\end{aligned}
$$

where $n$ is the topological degree of the map of the loop $\left(i_{1} i_{2} \cdots i_{p} i_{1}\right)$ into the circular loop (1231) and $m-3 n$ is a nonnegative even integer $2 u$. (The only terms having $\varphi$ or $\bar{\varphi}$ have $\eta i$ with them.) Write $\gamma=R_{j_{1}} \cdots R_{j_{k}}$. Then $\operatorname{Tr} \gamma$ is a sum of terms of the form $\eta^{2 p}(\bar{\eta} i)^{m-n}\left(\bar{\eta} i \varphi^{3}\right)^{n} \quad$ with $\quad(\bar{\eta} i)^{m-n}=(\bar{\eta} i)^{2(u+n)}=\bar{\eta}^{2(u+n)}(-1)^{u+n}$. Hence $\operatorname{Tr} \gamma \in \boldsymbol{Q}\left(\eta^{2}, \bar{\eta} i \varphi^{3}\right)$. But $\bar{\eta} i \varphi^{3}=e^{2 \pi i / \rho}$ for $\arg \varphi^{3}>-(\pi / 2-\pi / p)$ and $\operatorname{Tr} \operatorname{Ad} \gamma=(\operatorname{Tr} \gamma)^{2}$ by $\S 4$. Consequently

$$
\boldsymbol{Q}[\operatorname{Tr} \operatorname{Ad} \Gamma] \subset \operatorname{Re} \boldsymbol{Q}\left(e^{2 \pi i / \rho}, e^{2 \pi i / p}\right) .
$$

In order to prove the converse inclusion, we consider $\operatorname{Tr} \operatorname{Ad} \gamma$ for $\gamma=R_{2} R_{1} R_{2} R_{3}$. By (9.1.6) the eigenvalues of $R_{2} R_{1} R_{2} R_{3}$ are $\eta^{3} i \varphi^{3}$, $\eta^{3} i \bar{\varphi}^{3},-\eta^{2}$ and therefore 


$$
\begin{aligned}
(\operatorname{Tr} \gamma)^{2} & =\left|(-\eta)^{2}\left(-\eta i \varphi^{3}-\eta i \bar{\varphi}^{3}+1\right)\right|^{2} \\
& =3+2 \operatorname{Re}\left(\bar{\eta} i \varphi^{3}+\bar{\eta} i \bar{\varphi}^{3}+\varphi^{6}\right) \\
& =3+2\left(\cos \frac{2 \pi}{\rho}+\cos \frac{2 \pi \nu}{\sigma}+\cos 2 \pi t\right)
\end{aligned}
$$

where $t=\pi^{-1} \arg \varphi^{3}$ and $\nu$ is an integer prime to $\sigma$, the order of $\bar{\eta} i \bar{\varphi}^{3}$, with $\nu=1$ for $|t|<1 / 2-1 / p$.

Additional elements of $Q[\operatorname{Tr} A d \Gamma]$ are $\cos 2 \pi / p$ and $\cos 6 \pi / \rho$ arising from $\operatorname{Tr} \operatorname{Ad} R_{1}$ and $\operatorname{Tr}\left(R_{1} R_{2} R_{3}\right)^{2}$ respectively (cf. (9.1.1)).

Let $K$ denote the field $\boldsymbol{Q}\left[e^{2 \pi i / \rho}, e^{2 \pi i / p}\right] ; K$ is generated by a primitive root of unity $z$, and its Galois group is given by automorphisms $z \rightarrow z^{m}$ for integers $m$ prime to the order of $z$. The Galois group of $K$, Gal $K$, is thus abelian and the subfield $Q[\operatorname{Tr} A d \Gamma]$ is stable under all automorphisms of $K$. Hence

$$
\cos \frac{2 m \pi}{\rho}+\cos \frac{2 m \nu \pi}{\sigma}+\cos 2 m \pi t \in \boldsymbol{Q}[\operatorname{Tr} \operatorname{Ad} \Gamma]
$$

for all $m$ such that $z \rightarrow z^{m}$ is in Gal $K$. Examining the cases in Tables 1 and 2 one by one, it is easy to verify that some linear combination of the foregoing elements in $\boldsymbol{Q}(\operatorname{Tr} \operatorname{Ad} \Gamma)$ yield each of $\cos 2 \pi / \rho$, $\cos 2 \pi n / \sigma$, and $\cos 2 \pi t$ except in the case $p=4, \rho=5 / 12$. In the latter case one computes

$$
\begin{aligned}
\operatorname{Tr} R_{1} R_{2} R_{1} R_{2} R_{3} & =\eta^{3}\left(\eta^{3} i \bar{\varphi}^{3}-\eta-1-\eta i \bar{\varphi}^{3}+\eta i \varphi^{3}\right) \\
& =\eta^{2}\left[-\left(\frac{3}{2}+\sqrt{3}\right)+i\left(1+\frac{\sqrt{3}}{2}\right)\right]
\end{aligned}
$$

thus $\boldsymbol{Q}(\operatorname{Tr} \operatorname{Ad} \Gamma)$ contains $-(\sqrt{3} / 2)=\cos (2 \pi n / \sigma)$ in this case too.

The field $K$ has as a primitive generator any element $z=e^{2 \pi i N / L}$ where $L$ is the least common multiple of $p$ and $\rho$ and $\operatorname{gcd}(N, L)=1$. Thus each element of the field $K$ has the form $\sum_{j=0}^{l-1} a_{j} z^{j}$ with $a_{j} \in \boldsymbol{Q}$, and each element of the field $\operatorname{Re} K$ has the form

$$
\sum_{j=0}^{l-1} a_{j} \cos \frac{2 \pi j N}{L}
$$

As is well known, $\cos j \theta$ is a polynomial in the powers of $\cos \theta$ with integer coefficients for any integer $j$. Thus

$$
\operatorname{Re} K=\boldsymbol{Q}\left[\cos \frac{2 \pi N}{L}\right] \text {. }
$$

On the other hand, we have

$$
\frac{1}{2}-\frac{1}{p}+t=\frac{2}{p} \arg \bar{\eta} i \bar{\varphi}^{3}=\pi\left(\frac{1}{2}-\frac{1}{p}-t\right)=\left(1-2\left(\frac{1}{p}+\frac{1}{\rho}\right)\right) .
$$


Hence $\sigma$, the order of $\bar{\eta} i \bar{\varphi}^{3}$ is the order of $e^{2 \pi i / p} \cdot 2 \pi i / \rho$ if $\sigma$ is even, and half that order if $\sigma$ is odd. Thus if $\sigma$ is even and $\operatorname{gcd}(p, \rho)=1$, then $\sigma=L$ and $\exp (2 \pi i \nu / \sigma)$ generates the field $K$. In these cases $\cos (2 \pi \nu / \sigma)$ generates $\operatorname{Re} K$. In the remaining cases of Tables 1 and 2 , $\exp 2 \pi i t$ generates $K$ and $\cos 2 \pi t$ generates Re $K$. As noted above, these generators for $\operatorname{Re} K$ are in $\operatorname{Re} Q[\operatorname{Tr} A d \Gamma]$. Hence $\operatorname{Re} K \subset$ $\operatorname{Re} \operatorname{Tr} \operatorname{Ad} \Gamma$. From this the lemma follows.

\subsection{Non-artithmetic lattices.}

It remains only to apply the criterion of $\S 4$ to the groups $\Gamma(\varphi)$ listed in Tables 1 and 2 of $\S 17.1$ to determine which of them are non-arithmetic lattices.

TABLE 3

\begin{tabular}{|c|c|c|c|c|c|c|c|}
\hline$p$ & $\rho$ & $t$ & $k$-gen. & $\# \mathrm{Cal} k$ & $\Delta$ & & Arith. \\
\hline 3 & 6 & $1 / 6$ & 1 & 1 & & & $\mathrm{~A}$ \\
\hline 3 & 7 & $5 / 42$ & $\cos \frac{\pi}{21}$ & 6 & $-\cos \frac{5 \pi}{42} /\left(3 \sin \frac{\pi}{3}\right)$ & $\sigma_{5}(\Delta)=-\cos \frac{25 \pi}{42} /\left(3 \sin \frac{5 \pi}{3}\right)<0$ & NA \\
\hline 3 & 8 & $1 / 12$ & $\cos \frac{\pi}{12}$ & 4 & $-\cos \frac{\pi}{12} /\left(3 \sin \frac{\pi}{3}\right)$ & $\sigma_{7}(\Delta)=-\cos \frac{7 \pi}{12} /\left(-3 \sin \frac{7 \pi}{3}\right)<0$ & NA \\
\hline 3 & 9 & $1 / 18$ & $\cos \frac{\pi}{9}$ & 3 & $-\cos \frac{\pi}{18} /\left(3 \sin \frac{\pi}{3}\right)$ & $\sigma_{5}(\Delta)>0, \sigma_{25}(\Delta)>0$ & A \\
\hline 3 & 10 & $1 / 30$ & $\cos \frac{\pi}{15}$ & 4 & $-\cos \frac{\pi}{30} /\left(3 \sin \frac{\pi}{3}\right)$ & $\sigma_{11}(\Delta)=-\cos \frac{11 \pi}{30} /\left(-3 \sin \frac{11 \pi}{3}\right)<0$ & NA \\
\hline 3 & 12 & 0 & $\cos \frac{\pi}{6}$ & 2 & $-1 / 3 \sin \frac{\pi}{3}$ & $\sigma_{5}(\Delta)>0$ & $\mathrm{~A}$ \\
\hline 4 & 4 & $1 / 4$ & 1 & 1 & -1 & & $\mathrm{~A}$ \\
\hline 4 & 5 & $3 / 20$ & $\cos \frac{\pi}{10}$ & 4 & $-\frac{1}{2}\left(1+\frac{\cos 3 \pi / 20}{\sin \pi / 4}\right)$ & $\sigma_{3}(\Delta)=-\frac{1}{2}\left(1+\frac{\cos 9 \pi / 20}{-\sin \pi / 4}\right)<0$ & NA \\
\hline 4 & 6 & $1 / 12$ & $\cos \frac{\pi}{6}$ & 2 & $-\frac{1}{2}\left(1+\frac{\cos \pi / 20}{\sin \pi / 4}\right)$ & $\sigma_{5}(\Delta)=-\frac{1}{2}\left(1+\frac{\cos 5 \pi / 12}{\sin 5 \pi / 4}\right)<0$ & NA \\
\hline 4 & 8 & 0 & $\cos \frac{\pi}{4}$ & 2 & $-\frac{1}{2}\left(1+\frac{1}{\sin \pi / 4}\right)$ & $\sigma_{5}(\Delta)>0$ & $\mathrm{~A}$ \\
\hline 5 & 4 & $1 / 5$ & $\cos \frac{\pi}{10}$ & 4 & $1-\frac{3}{4 \sin ^{2} \pi / 5}-\frac{\cos \pi / 5}{4 \sin ^{3} \pi / 5}$ & $\sigma_{11}(\Delta)=1-2.1708+.9959<0$ & NA \\
\hline 5 & 5 & $1 / 10$ & $\cos \frac{\pi}{5}$ & 2 & $1-\frac{3}{4 \sin ^{2} \pi / 5} \frac{\cos \pi / 10}{4 \sin ^{3} \pi / 5}$ & $\sigma_{3}(\Delta)>0$ & $\mathrm{~A}$ \\
\hline 3 & 4 & $1 / 3$ & $\cos \frac{\pi}{6}$ & 2 & $-\frac{1}{2 \cdot 3 \sin \pi / 3}$ & $\sigma_{5}(\Delta)>0$ & A \\
\hline 3 & 5 & $7 / 30$ & $\cos \frac{2 \pi}{15}$ & 4 & $-\frac{\cos 7 \pi / 30}{3 \sin \pi / 3}$ & $\sigma_{7}(\Delta)>0, \quad \sigma_{7^{2}}(\Delta)>0, \quad \sigma_{7^{2}}(\Delta)>0$ & A \\
\hline 4 & 3 & $5 / 12$ & $\cos \frac{\pi}{6}$ & 2 & $-\frac{1}{2}\left(1+\frac{\cos 5 \pi / 12}{3 \sin \pi / 4}\right)$ & $\sigma_{5}(\Delta)>0$ & $\mathrm{~A}$ \\
\hline 5 & 2 & $7 / 10$ & $\cos \frac{\pi}{5}$ & 2 & $1-\frac{3}{4 \sin ^{2} \pi / 5}+\frac{\cos 3 \pi / 10}{4 \sin ^{3} \pi / 5}$ & $\sigma_{3}(\Delta)>0$ & $\mathrm{~A}$ \\
\hline 5 & 3 & $11 / 30$ & $\cos \frac{2 \pi}{15}$ & 4 & $1-\frac{3}{4 \sin ^{2} \pi / 5}-\frac{\cos 11 \pi / 30}{4 \sin ^{3} \pi / 5}$ & $\sigma_{49}(\Delta)=1-2.17-\frac{\cos 539 \pi / 30}{4 \sin ^{3} 49 \pi / 5}>0$ & NA \\
\hline
\end{tabular}


All the groups of $\S 17.1$ are discrete. Except for the two cases $(p, \rho)=(3.6)$ and (4.4) in which $\sigma=\infty$, the regions $\Omega(\varphi)$ are compact and therefore $P U(H(\varphi)) / \Gamma(\varphi)$ is compact; in the other two cases, $\Omega(\varphi)$ has three cusps at the boundary of the ball but nevertheless has finite measure. Accordingly $\Gamma$ is a lattice subgroup of $P U(H)$ for all the groups listed in Tables 1 and 2 .

We list the arithmeticity results in Table 3 . The fourth column lists a primitive generator of the field $k=\boldsymbol{Q}[\mathrm{Ad} \Gamma]$, the fifth column lists the order of the Galois group of $k . \quad \Delta=1-3 \alpha^{2}-\alpha^{3}\left(\varphi^{3}+\bar{\varphi}^{3}\right)$, the determinant of the matrix $\left\langle e_{i}, e_{j}\right\rangle(i, j=1,2,3)$. The sixth column lists the effect of the automorphism $\sigma_{n}: z \rightarrow z^{n}$ an $\Delta$, where $z$ is a primitive generator of the cyclotomic field containing $\eta^{2}, i, \varphi^{3}$, and the value $\sigma_{n}(\Delta)$ for some $n$ when $\sigma_{n}(\Delta)<0$ and $\sigma_{n} \neq$ identity on $k=$ $\boldsymbol{Q}[\operatorname{Tr} \operatorname{Ad} \Gamma]$. In the last column $\mathrm{A}$ and $\mathrm{NA}$ denote arithmetic and non-arithmetic respectively.

In our situation, $\Delta=1-3 /\left(4 \sin ^{2}(\pi / p)\right)-\cos \pi t /\left(4 \sin ^{3}(\pi / p)\right)$ which simplifies to $-(\cos \pi t /) /(3 \sin \pi / 3),-(1 / 2)(1+(\cos \pi t) /(\sin \pi / 3))$ for $p=$ 3,4 respectively.

Summing up the information contained in Table 3, we get the following result.

THEOREM 17.3. There exists in $P U(2,1)$ a non-arithmetic lattice generated by $\boldsymbol{C}$-reflections of order 3,4 , or 5 . Up to an isometry, any such lattice with Coxeter diagram

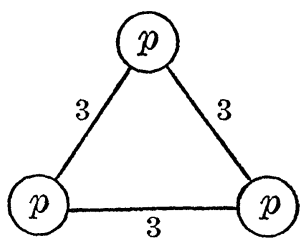

and $\left\langle e_{1}, e_{2}\right\rangle\left\langle e_{2}, e_{3}\right\rangle\left\langle e_{3}, e_{1}\right\rangle=e^{\pi 2 t} i s$ given by the seven values

$$
\begin{aligned}
(p, t)= & (3,5 / 42),(3,1 / 12),(3,1 / 30) \\
& (4,3 / 20),(4,1 / 12) \\
& (5,1 / 5),(5,11 / 30)
\end{aligned}
$$

The non co-compact lattices $\Gamma(\varphi)$ are arithmetic.

18. The space $Y(\varphi),\left|\arg \varphi^{3}\right|<\pi / 2-\pi / p,(\arg \varphi) / \pi \in \boldsymbol{Q}$.

18.1. The joined space.

We have seen in $\$ 14.2$ that for $\left|\arg \varphi^{3}\right|<\pi / 2-\pi / p$, the region $\Omega(\varphi)$ is bounded by the 24 3-dimensional faces 


$$
\left.E_{1}(\Omega)=\left\{\widetilde{R}_{i}^{ \pm 1}, R_{i}, \widetilde{R}_{j}\right)^{ \pm 1},\left(R_{i} R_{j} \widetilde{R}_{i}\right)^{ \pm 1} ; i, j=1,2,3 ; i \neq j\right\}
$$

and that for each $\gamma \in \Gamma$,

$$
\gamma: \tilde{\gamma} \longrightarrow \tilde{\gamma}^{-1} \text {. }
$$

Set $\Delta(\varphi)=\left\{R_{i}^{ \pm 1},\left(R_{i} R_{j}\right)^{ \pm 1},\left(R_{i} R_{j} R_{i}\right)^{ \pm 1} ; i, j=1,2,3 ; i \neq j\right\}$ and let $\Gamma(\varphi)$ denote the subgroup of Isom $B$ generated by $\Delta(\varphi)$. Write $F, \Delta, \Gamma$ for $\Omega(\varphi), \Delta(\varphi)$, and $\Gamma(\varphi)$ respectively. For any $\gamma \in \Delta$, set

$$
e(\gamma)=\tilde{\gamma}^{-1}, \quad T(\tilde{\gamma})=\gamma^{-1} .
$$

By the proposition of $\S 14.4$, we may assert

$$
\gamma(F) \cap F=e(\gamma) \text {, all } \gamma \in \Delta .
$$

Set $\mathscr{F}-\Gamma F$. By Theorem 6.3.2(I), $\mathscr{F}$ is a connected abutted family of polyhedra. We let $\mathscr{N}$ denote the adjacency of $\mathscr{F}$ and we let $Y(\varphi)$ denote the joined $\mathscr{F}$-space. In $\S 14.3$ we have seen that the polyhedron $F$ has 15 vertices of 4 distinct types, up to equivalence. Mod Aut $F$

$$
\begin{array}{lll}
p_{i j}(i \neq j) & \text { with } & p_{i j}=p_{j i} \\
s_{i j}(i \neq j) & \text { with } & s_{i j}=s_{j i} \\
\widetilde{s}_{i j}(i \neq j) & \text { with } & \widetilde{s}_{i j}=\widetilde{s}_{j i} \\
t_{i j}(i \neq j) . & &
\end{array}
$$

We recapitulate here the results from $\S 16$. Let

$$
\begin{aligned}
& r=\operatorname{order}\left(R_{1} R_{2} R_{3}\right)^{2}=\operatorname{order} \eta^{3} i \bar{\varphi}^{9}=\operatorname{order}(\bar{\eta} i \varphi)^{3} \\
& s=\operatorname{order}\left(R_{3} R_{2} R_{1}\right)^{2}=\operatorname{order} \eta^{3} i \varphi^{9}=\operatorname{order}\left(\bar{\eta} i \bar{\varphi}^{3}\right)^{3} \\
& \rho=\operatorname{order} \bar{\eta} i \varphi^{3} \\
& \sigma=\operatorname{order} \bar{\eta} i \bar{\varphi}^{3} .
\end{aligned}
$$

These integers are finite if and only if $(2 \pi)^{-1} \arg \varphi \in \boldsymbol{Q}$. In this case, $\Gamma_{F}=Z_{3}$ if either $\rho=r$ or $\sigma=s$; equivalently 3 does not divide $\rho$ or $\sigma$. Cyclic permutations of indices make up Aut $F$ for all $\varphi \neq 1$. Thus for $\varphi \neq 1$, Aut $F=Z_{3}$. If $\varphi=1, F$ is stable under all permutations and Aut $F=Z_{\mathrm{\varepsilon}}$. Set $\Gamma_{\alpha}=(\operatorname{Aut} \Gamma) \Gamma$. Then modulo $\Gamma_{\alpha}$, the vertices of $E_{1}(F)$ are

$$
\begin{aligned}
& E_{4}(F) / \Gamma_{\alpha}: p_{12}, s_{12}, t_{32}, t_{23} \\
& \text { modulo } \Gamma_{\alpha}, \text { the remaining }(4-k) \text {-faces } E_{k}(F) \text { are } \\
& E_{3}(F) / \Gamma_{\alpha}: p_{12} t_{32}, p_{12}, t_{23}, p_{12} s_{12}\left(\text { via } s_{12}^{*}\right), p_{12} s_{12}\left(\text { via } s_{21}^{*}\right) \\
& \quad s_{12} t_{32}, s_{31} t_{23}, \\
& \quad \widetilde{s}_{13} s_{12}, \widetilde{s}_{21} s_{31}
\end{aligned}
$$




$$
E_{2}(F) / \Gamma_{\alpha}: e_{1}^{\perp} \cap F, \Delta_{123}, \Delta_{321}
$$

(18.1.2) $\quad \widetilde{R}_{1} \cap \widetilde{R}_{1} R_{2}, \widetilde{R}_{2} \cap \widetilde{R}_{1} R_{2}, \widetilde{R}_{1} \cap R_{1} \widetilde{R}_{2} R_{1}, \widetilde{R}_{2} \cap R_{1} \widetilde{R}_{2} R_{1}, R_{1} \widetilde{R}_{2} \cap R_{1} \widetilde{R}_{2} R_{1}$, $\widetilde{R}_{2} R_{1} \cap R_{1} \widetilde{R}_{2} R_{1}$

$$
E_{1}(F) / \Gamma_{\alpha}: \widetilde{R}_{1}, \widetilde{R}_{1} R_{2}, \widetilde{R}_{2} R_{1}, R_{1} \widetilde{R}_{2} R_{1} .
$$

\subsection{Stabilizers.}

Up to an automorphism of $\Gamma$, the stabilizers of all faces in the polyhedral decomposition $\mathscr{F}$ of the space $Y(\varphi)$ is given by the stabilizers of the faces $\eta(e, F)$ where $\eta$ denotes the canonical map of $F \times \mathscr{F}$ onto $Y(\varphi)$ (cf. $\S 6.2$ ) and $e$ ranges over the faces in the list (18.1). As above, $\Gamma(e, F)$ denotes the stabilizer of the face $\eta(e, F)$.

(18.2.1) $\Gamma_{\left(p_{12}, F\right)}$ is the subgroup $\Gamma_{12}$ of Aut $B(=\mathrm{PU}(H))$ generated by $R_{1}$ and $R_{2}$; it has order $24(p /(6-p))^{2}$.

Proof. Let $\gamma \in \Gamma_{\left(p_{12}, F\right)}$. Then $\gamma p_{12}=p_{12}$ and $\gamma F$ is $p_{12}$-connected to $F$ in $\mathscr{F}$, and $\gamma F \in \mathscr{F}_{p_{12}, F}$. By Remark 2 of $\S 6.6, \mathscr{F}_{p_{12}, F}=\Gamma_{12} F_{p_{12}}$. Since $F$ lies in a fundamental domain for the action of $\Gamma_{12}$ on $B, \Gamma_{12}$ operates simply transitively on $\mathscr{F}_{p_{12}, F}$ and $\gamma \in \Gamma_{12}$. The order of $\Gamma_{12}$ is given in $\S 2$.

Proposition 18.2.3.

(i ) $\quad \Gamma_{\left(s_{12}, F^{\prime}\right)}=\left\{\left\{R_{3} R_{1} R_{2} R_{1}, R_{1}^{-1} R_{3} R_{1} R_{2}\right\}\right\}=\left\{\left\{R_{3} R_{2} R_{1} R_{2}, R_{2}^{-1} R_{3} R_{2} R_{1}\right\}\right\}$ $\Gamma_{\left(\tilde{s}_{12}, F\right)}=\left\{\left\{R_{1} R_{2} R_{1} R_{3}, R_{2} R_{1} R_{3} R_{1}^{-1}\right\}\right\}=\left\{\left\{R_{2} R_{1} R_{2} R_{3}, R_{1} R_{2} R_{3} R_{2}^{-1}\right\}\right\}$ and these stabilizers are abelian.

(ii) (a) If $3 \nmid \rho \sigma$, then $\Gamma_{\left(s_{12}, F^{\prime}\right)}=\left\{\left\{\left(R_{3} R_{1} R_{2}\right)^{2},\left(R_{3} R_{2} R_{1}\right)^{3}\right\}\right\}=Z_{r} \times \boldsymbol{Z}_{s}$.

(b) If $3 \mid \rho \sigma$, then $\left.\left\{\left\{\left(R_{3} R_{1} R_{1}\right)^{2},\left(R_{3} R_{2} R_{1}\right)\right\}^{2}\right\}\right\}$ is a subgroup of index 3 in $\Gamma_{\left(s_{12}, F\right)}$.

(iii) $\Gamma_{\left(s_{12}, F\right)} \mid G\left(s_{12}, F\right)$ has 6 elements represented by

$$
\text { 1, } R_{1}^{-1}, R_{2}^{-1}, R_{3}, R_{3} R_{1}, R_{3} R_{2} \text {. }
$$

(iv) $\Gamma_{\left(s_{12}, F\right)} \mid \mathscr{F}_{s_{12}, F}$ has $\begin{cases}2 \text { elements represented by } F, R_{3} F \\ & \text { if } \Gamma_{F} \neq\{1\} \\ 6 \text { elements } & \text { if } \Gamma_{F}=1 .\end{cases}$

Proof. Set $a=R_{3} R_{1} R_{2} R_{1}, b=R_{1}^{-1} R_{3} R_{1} R_{2}, c=b^{-1} a . \quad$ Then $c=$ $R_{2}^{-1} R_{1}^{-1} R_{3}^{-1}\left(R_{1} R_{3} R_{1}\right) R_{2} R_{1}=R_{2}^{-1} R_{1}^{-1} R_{3}^{-1} R_{3} R_{1} R_{3} R_{2} R_{1}=R_{2}^{=1} R_{3} R_{2} R_{1}$. Set $\Gamma^{1}=$ $\{\{a, b\}\}$. Then $\Gamma^{1}=\{\{a, c\}\}=\left\{\left\{R_{3} R_{2} R_{1} R_{2}, R_{2}^{-1} R_{3} R_{2} R_{1}\right\}\right\}$.

We shall prove (i) with the help of Proposition 6.6. By $\S 14$, the 3-faces of $F$ containing $s_{12}$ are

$$
\widetilde{R}_{1}, \widetilde{R}_{2}, \widetilde{R}_{3}^{-1}, R_{1} \widetilde{R}_{2}, \widetilde{R}_{2} R_{1},\left(R_{3} \widetilde{R}_{1}\right)^{-1},\left(R_{3} \widetilde{R}_{2}\right)^{-1}, R_{1} \widetilde{R}_{2} R_{1} .
$$


Thus the adjacent cells $\mathscr{N}\left(F_{s_{12}}\right)$ are of the form $(\gamma F)_{s_{12}}$ with $\gamma$ in the subset of $\Delta$ denoted

$$
F\left[s_{12}\right]: R_{1}^{-1}, R_{2}^{-1}, R_{3},\left(R_{1} R_{2}\right)^{-1},\left(R_{2} R_{1}\right)^{-1}, R_{3} R_{1}, R_{3} R_{2},\left(R_{1} R_{2} R_{1}\right)^{-1} .
$$

We first verify

$$
\mathscr{N}\left(\mathscr{N}\left(F_{s_{12}}\right)\right) \subset \Gamma^{1}\left(\mathscr{N}\left(F_{s_{12}}\right) \cup F_{s_{12}}\right) .
$$

In verifying this, we make use of the evident fact: for any $\gamma \in I$, $F \in \mathscr{F}, e \in E_{l}(\gamma F)$

$$
\mathscr{N}\left((\gamma F)_{e}\right)=\gamma \mathscr{N}\left(F_{i-1}\right) .
$$

In order to prove (1), we must show for each $\gamma \in F\left[s_{12}\right]$ and $\delta \in$ $F\left[\gamma^{-1} s_{12}\right]$ that

$$
\gamma \delta \hat{\delta} \in \Gamma^{1}\left(F\left[s_{12}\right] \cup 1\right) .
$$

For $\gamma=R_{1}^{-1}$, we find $\gamma^{-1} s_{12}=R_{1} s_{12}=\widetilde{s}_{31}$. From $\S 11$, we find the 3faces of $F$ containing $\widetilde{s}_{31}$ and accordingly we get for

$$
F\left[\widetilde{s}_{31}\right]: R_{3}, R_{1}, R_{3} R_{1}, R_{1} R_{3}, R_{1} R_{3} R_{1}, R_{2}^{-1},\left(R_{1} R_{2}\right)^{-1},\left(R_{3} R_{2}\right)^{-1}
$$

and $R_{1}^{-1} F\left[\widetilde{s}_{31}\right]=R_{1}^{-1} R_{3}, 1, R_{1}^{-1} R_{3} R_{1}, R_{3}, R_{3} R_{1},\left(R_{2} R_{1}\right)^{-1},\left(R_{1} R_{2} R_{1}\right)^{-1},\left(R_{3} R_{2} R_{1}\right)^{-1}$. We have $R_{1}^{-1} R_{3} R_{1} R_{2}=b \in \Gamma^{1}$. Thus

$$
R_{1}^{-1} R_{3}=b\left(R_{1} R_{2}\right)^{-1} \subset \Gamma^{1} F\left[s_{12}\right] .
$$

Similarly $R_{1}^{-1} R_{3} R_{1}=\left(R_{1}^{-1} R_{3} R_{1} R_{2}\right) R_{2}^{-1} \subset \Gamma^{1} F\left[s_{12}\right]$, and

$$
\left(R_{3} R_{2} R_{1}\right)^{-1}=\left(R_{2}^{-1} R_{3} R_{2} R_{1}\right)^{-1} R_{2}^{-1} \subset \Gamma^{1} F\left[s_{12}\right] .
$$

Consequently $R_{1}^{-1} F\left[\widetilde{s}_{31}\right] \subset \Gamma^{1}\left(F\left[s_{12}\right] \cup 1\right)$. The verification of $\left(1^{\prime}\right)$ for $\gamma=$ $R_{2}^{-1}$ and $R_{3}$ is similar. For $\gamma=\left(R_{1} R_{2}\right)^{-1}$, we find $\left(R_{1} R_{2}\right) s_{12}=s_{23}$, and

$$
\begin{aligned}
& F\left[s_{23}\right]: R_{2}^{-1}, R_{3}^{-1}, R_{1},\left(R_{2} R_{3}\right)^{-1},\left(R_{3} R_{2}\right)^{-1}, R_{1} R_{2}, R_{1} R_{3},\left(R_{2} R_{3} R_{2}\right)^{-1} \\
& \left(R_{1} R_{2}\right)^{-1} F\left[s_{23}\right]:\left(R_{1} R_{2} R_{1}\right)^{-1},\left(R_{3} R_{1} R_{2}\right)^{-1}, R_{2}^{-1},\left(R_{2} R_{3} R_{1} R_{2}\right)^{-1}\left(R_{3} R_{2} R_{1} R_{2}\right)^{-1}, \\
& 1, R_{2}^{-1} R_{3},\left(R_{2} R_{3} R_{2} R_{1} R_{2}\right)^{-1} \\
& \quad R_{2}^{-1} R_{3}=R_{2}^{-1} R_{3} R_{2} R_{1}\left(R_{2} R_{1}\right)^{-1} \in \Gamma^{1} F\left[s_{12}\right] .
\end{aligned}
$$

Similarly, $\left(R_{2} R_{3} R_{2} R_{1} R_{2}\right)^{-1}=a^{-1} R_{2}^{-1} \in \Gamma^{1} F\left[s_{12}\right]$. Verification of (1') for $\gamma=\left(R_{2} R_{1}\right)^{-1}, R_{3} R_{1}, R_{3} R_{1}$, and $\left(R_{1} R_{2} R_{1}\right)^{-1}$ is similar. For example, $R_{1} R_{2} R_{1} s_{12}=\widetilde{s}_{12}$ and we have for

$$
\begin{aligned}
& F\left[\widetilde{s}_{12}\right]: R_{1}, R_{2}, R_{1} R_{2}, R_{2} R_{1}, R_{2} R_{1} R_{2}, R_{3}^{-1}, R_{2} \\
& \left(R_{1} R_{2} R_{1}\right)^{-1} F\left(\widetilde{s}_{12}\right):\left(R_{2} R_{1}\right)^{-1},\left(R_{1} R_{2}\right)^{-1}, R_{1}^{-1}, R_{2}^{-1}, 1,\left(R_{3} R_{1} R_{2} R_{1}\right)^{-1}, \quad \text { and } \\
& \quad\left(R_{2} R_{3} R_{1} R_{2} R_{1}\right)^{-1}=a^{-1} R_{2}^{-1},\left(R_{1} R_{3} R_{1} R_{2} R_{1}\right)^{-1}=a^{-1} R_{1}^{-1}
\end{aligned}
$$

all in $\Gamma^{1}\left(F\left[s_{12}\right] \cup 1\right)$, in verification of $\left(1^{\prime}\right)$. 
Next we compute $S=\left(\{1\} \cup F\left[s_{12}\right]\right) T_{F} \cap \Gamma_{\left(s_{12}, F\right)}$. Let $J$ denote the automorphism of $B$ arising from the cyclic permutation $(1,2,3) \rightarrow$ $(2,3,1)$. Then Aut $F=\{\{J\}\}$. From $\S 16$ we know that if 3 does not divide $\rho$,

$$
J=\left(R_{1} R_{2} R_{3}\right)^{2 \mu} R_{1} R_{2}=\left(R_{2} R_{3} R_{1}\right)^{2 \mu} R_{2} R_{3}=\left(R_{3} R_{1} R_{2}\right)^{2 \nu} R_{3} R_{1}
$$

where $3 \mu+1 \equiv 0(\bmod \rho)$; if 3 does not divide $\sigma$

$$
J^{-1}=\left(R_{3} R_{2} R_{1}\right)^{2 \nu} R_{3} R_{2}=\left(R_{2} R_{1} R_{3}\right)^{2 \nu} R_{2} R_{1}=\left(R_{1} R_{3} R_{2}\right)^{2 \nu} R_{1} R_{3}
$$

where $3 \nu+1 \equiv 0(\bmod \sigma)$; if $3 \mid \rho$ and $3 \mid \sigma$ then $\Gamma_{F}=(1)$.

From the relation of $\S 13$ :

$$
R_{i} s_{i j}=\widetilde{s}_{i k}, R_{k} \widetilde{s}_{i j}=s_{i j}(i \neq j)\left(s_{i j}=s_{j i}\right) .
$$

These permit us to find

$$
\begin{aligned}
& R_{1} s_{12}=\widetilde{s}_{13}, R_{2} s_{12}=\widetilde{s}_{32}, R_{3} \widetilde{s}_{21}=s_{12} \\
& R_{1} R_{2} s_{12}=s_{23}, R_{2} R_{1} s_{12}=s_{31}, R_{3} R_{1} s_{31}=s_{12}, R_{3} R_{2} s_{23}=s_{12} R_{1} R_{2} R_{1} s_{12}=\widetilde{s}_{21} .
\end{aligned}
$$

Consequently if

[A]

[B]

$$
\begin{aligned}
& \Gamma_{F}=(1), S=\left\{\gamma \in\{1\} \cup F\left[s_{12}\right] ; \gamma s_{12}=s_{12}\right\}=(1) \\
& \Gamma_{F}=\{\{J\}\}, S=\left\{1,\left(R_{1} R_{2}\right)^{-1} J,\left(R_{2} R_{1}\right)^{-1} J^{-1}, R_{3} R_{1} J^{-1}, R_{3} R_{2} J\right\} .
\end{aligned}
$$

If $3 \nmid \rho,\left(R_{1} R_{2}\right)^{-1} J=R_{2}^{-1} R_{1}^{-1}\left(R_{1} R_{2} R_{3}\right)^{2 \mu} R_{1} R_{2}=\left(R_{3} R_{1} R_{2}\right)^{2 \mu} \in \Gamma^{1}$. If $3 \nmid \sigma$, $\left(R_{1} R_{2}\right)^{-1} J=\left(R_{1} R_{2}\right)^{-1}\left(R_{3} R_{2}\right)^{-1}\left(R_{3} R_{2} R_{1}\right)^{-2 \nu}=\left(R_{3} R_{2} R_{1} R_{2}\right)^{-1}\left(R_{3} R_{2} R_{1}\right)^{-2 \nu} \in \Gamma^{1}$. Similarly, we find in Case [B] that $S \subset \Gamma^{1}$. Hence $\Gamma^{1}=\Gamma_{\left(s_{12}, F\right)}$. By Proposition 6.6. $\Gamma_{\left(s_{12}, F\right)}=\Gamma^{1} S=\Gamma^{1}$. The result for $\Gamma_{\left(\widetilde{s_{12}}, F\right)}$ follows from the isomorphism of $\Gamma(\varphi)$ to $\Gamma(\bar{\varphi})$ provided by complex conjugation which sends $s_{i j}(\varphi)$ to $\widetilde{s}_{i j}(\bar{\varphi})$ and $R_{i}(\varphi)$ to $R_{i}^{-1}(\bar{\varphi})$.

Proof of (ii). $a c=\left(R_{3} R_{2} R_{1}\right)^{2}$ and $b c=R_{1}^{-1} R_{3} R_{1} R_{3} R_{2} R_{1}=R_{1}^{-1} R_{1} R_{3} R_{1} R_{2} R_{1}=$ $a$. Therefore $a^{3}=a b \cdot a c \in \Gamma^{1}$. Let $E$ denote the subgroup $\{\{a b, a c\}\}$. Then $\Gamma^{1} / E$ has order dividing 3 . By $\S 10$ the matrix $R_{3} R_{1} R_{2} R_{1}$ is diagonalizable with eigenvalues $\left(\eta^{3} i \bar{\varphi}^{3}, \eta^{3} i \varphi^{3},-\eta^{2}\right)$ so that its order in $\operatorname{PU}(H)$ is the order of the diagonal matrix $d=\left(-\eta i \bar{\varphi}^{3},-\eta i \varphi^{3}\right)$. On the other hand, by $\S 10$, the order $\left(R_{3} R_{1} R_{2}\right)^{2}$ in $\mathrm{PU}(H)$ is the order of $\eta^{3} i \bar{\varphi}^{9}=$ $\left(-\eta i \bar{\varphi}^{3}\right)^{3}$ and the order of $\left(R_{3} R_{2} R_{1}\right)^{2}=\left(-\eta i \varphi^{3}\right)^{3}$. If 3 does not divide the order of $d$, then $a^{3}$ has the same order as $a$ and $a^{3} \in E$ implies that $a \in E$. Therefore $E=\Gamma^{1}$, and $\Gamma_{\left(s_{12}, F\right)}=\left\{\left(R_{3} R_{1} R_{2}\right)^{2},\left(R_{3} R_{2} R_{1}\right)^{2}\right\}$. The intersection of the cyclic groups $\left\{\left(R_{3} R_{1} R_{2}\right)^{2} \cap\left\{\left(R_{3} R_{2} R_{1}\right)^{2}\right\}=(1)\right.$ for any element in this intersection fixes each point of the orthogonal $C$-lines containing $\Delta_{312}$ and $\Delta_{321}$ respectively and therefore fixes all the points of $B$. Thus $\Gamma_{\left(s_{12}, F\right)}=Z_{r} \times Z_{s}$ in Case 3 does not divide the order of $d$, or equivalently the order of $\bar{d}=\left(\bar{\eta} i \varphi^{3}, \bar{\eta} i \bar{\varphi}^{3}\right)$. This proves (iia). 
If 3 divides the order of $d$, then 3 divides the order of $a$ so that $a$ is not in $E$. Hence $E$ is of index 3 in $\Gamma_{\left(s_{12}, F\right)}$, proving (iib).

Proof of (iii). Write $\Gamma^{1}$ for $\Gamma_{\left(s_{12}, F\right)}, \mathscr{F}^{1}$ for $\mathscr{F}_{s_{12}, F}$. We know from the proof of (i) that

$$
G\left(s_{12}, F\right)=\Gamma^{1}\left(\{1\} \cup F\left[s_{12}\right]\right) F .
$$

Clearly

$$
\begin{aligned}
& \left(R_{3} R_{1} R_{2} R_{1}\right)^{-1} R_{3} R_{1}=\left(R_{2} R_{1}\right)^{-1} \\
& \left(R_{3} R_{2} R_{1} R_{2}\right)^{-1} R_{3} R_{2}=\left(R_{1} R_{2}\right)^{-1} \\
& \left(R_{3} R_{2} R_{1} R_{2}\right)\left(R_{1} R_{2} R_{1}\right)^{-1}=R_{3} .
\end{aligned}
$$

Consequently, $G\left(s_{12}, F\right)=\Gamma^{1}\left\{1, R_{1}^{-1}, R_{2}^{-1}, R_{3}, R_{3} R_{1}, R_{3} R_{2}\right\}$. For any two distinct elements $\gamma_{1}, \gamma_{2}$ of the six elements $Q=\left\{1, R_{1}^{-1}, R_{2}^{-1}, R_{3}, R_{3} R_{1}\right.$, $R_{3} R_{2}$ \} one can order them so that $\gamma_{1} \gamma_{2}^{-1}$ is either in $Q$ or is one of $\left\{R_{1}^{-1} R_{2}, R_{3} R_{1}^{2} R_{3} R_{2}^{2} R_{3} R_{1} R_{2} R_{3} R_{2} R_{1} R_{3} R_{1} R_{2}^{-1} R_{3}^{-1}\right\}$. From $\S 13$ we have the diagrams

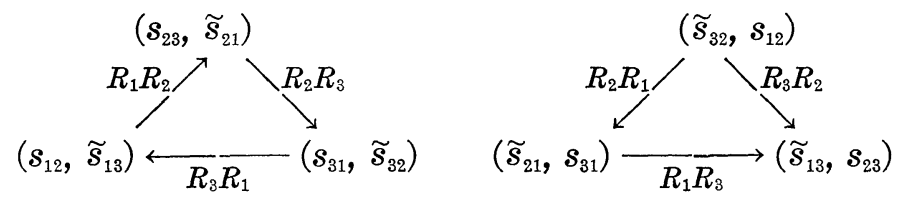

and also

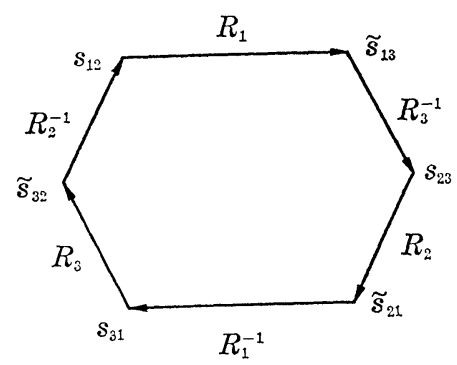

From these relations it is easy to verify that for any $\gamma_{1}, \gamma_{2} \in Q$, $\gamma_{1} \gamma_{2}^{-1} s_{12} \neq s_{12}$. For example

$$
R_{3} R_{1} R_{2}^{-1}\left(s_{12}\right)=R_{3} R_{1}\left(s_{23}\right) \neq s_{12}
$$

since $\left(R_{3} R_{1}\right)^{-1} s_{12}=s_{31} \neq s_{23}$. Hence $G\left(s_{12}, F\right)$ has exactly six $\Gamma_{\left(s_{12}, F\right)}$ orbits.

Proof of (iv). The set $\left.\Gamma_{\left(s_{12}, F\right)}\right) \mathscr{F}_{\mathrm{s}_{12}, F}$ is the space of double cosets $\left.\Gamma_{\left(s_{12}, F\right)}\right) G\left(s_{12}, F\right) / \Gamma_{F}$. It remains therefore to find which pairs of ele- 
ments $g_{1}$ and $g_{2}$ of $Q$ are in the same double coset; this is the case if and only if

$$
g_{2} J^{ \pm 1} g_{1} \in \Gamma_{\left(s_{12}, F\right)} \quad\left(=\Gamma^{1}\right) .
$$

Clearly if $\Gamma_{F}=\{1\}$, then $\mathscr{F}_{s_{2}, F}$ is represented by the six elements of $G\left(s_{12}, F\right)$. If $\Gamma_{F} \neq\{1\}$, then either $3 \nmid \rho$ or $3 \nmid \sigma$.

Case 1. $3 \nmid \rho$. Then

$$
\begin{aligned}
& R_{3} R_{1} \cdot J^{-1} \cdot 1=R_{3} R_{1}\left[\left(R_{3} R_{1} R_{2}\right)^{2 \mu} R_{3} R_{1}\right]^{-1} \in \Gamma^{1} \\
& \begin{aligned}
R_{3} R_{2} \cdot J \cdot 1 & =R_{3} R_{2}\left[\left(R_{1} R_{2} R_{3}\right)^{2 \mu} R_{1} R_{2}\right]=R_{3}\left(R_{1} R_{2} R_{3}\right)^{2 \mu} R_{2} R_{1} R_{2} \\
& =\left(R_{3} R_{1} R_{2}\right)^{2 \mu} R_{3} R_{2} R_{1} R_{2} \in \Gamma^{1}
\end{aligned} \\
& \begin{aligned}
R_{3} J R_{1}= & R_{3}\left(R_{1} R_{2} R_{3}\right)^{2 \mu} R_{1} R_{2} R_{1}=\left(R_{3} R_{1} R_{2}\right)^{2 \mu} R_{3} R_{1} R_{2} R_{1} \in \Gamma^{1} \\
R_{3} J^{-1} R_{2} & =R_{3}\left[\left(R_{2} R_{3} R_{1}\right)^{2 \mu} R_{2} R_{3}\right]^{-1} R_{2}=R_{3}\left[R_{2}\left(R_{3} R_{1} R_{2}\right)^{2 \mu} R_{3}\right]^{-1} R_{2} \\
& =\left(R_{3} R_{1} R_{2}\right)^{2 \mu} \in \Gamma^{1} .
\end{aligned}
\end{aligned}
$$

Case 2. $3 \nmid \sigma$

$$
\begin{aligned}
& R_{3} R_{1} \cdot J^{-1} \cdot 1=R_{3}\left(R_{2} R_{1} R_{3}\right)^{2 \nu} R_{1} R_{2} R_{1}=\left(R_{3} R_{2} R_{1}\right)^{2 \nu} R_{3} R_{1} R_{2} R_{1} \in \Gamma^{1} \\
& R_{3} R_{2} J \cdot 1=R_{3} R_{2}\left[\left(R_{3} R_{2} R_{1}\right)^{2 \nu} R_{3} R_{2}\right]^{-1} \in \Gamma^{1} \\
& R_{3} J R_{1}=R_{3}\left[\left(R_{1} R_{3} R_{2}\right)^{2 \nu} R_{1} R_{3}\right]^{-1} R_{1}=\left[R_{1}\left(R_{3} R_{2} R_{1}\right)^{2 \nu}\right]^{-1} R_{1} \in \Gamma^{1} \\
& R_{3} J R_{2}=R_{3}\left[\left(R_{2} R_{1} R_{3}\right)^{2 \nu} R_{2} R_{1}\right] R_{2}=\left(R_{3} R_{2} R_{1}\right)^{2 \nu} R_{3} R_{2} R_{1} R_{2} \in \Gamma^{1} .
\end{aligned}
$$

Thus if either $3 \nmid \rho$ or $3 \nmid \sigma$, the space of double cosets has at most 2 distinct elements represented by 1 and $R_{3}$. It is easily verified that $R_{3} J^{ \pm 1} s_{12} \neq s_{12}$. Proof of (iv) is now complete.

Proposition 18.2.4. Let $\rho=\operatorname{order} \bar{\eta} i \bar{\varphi}^{3}, \quad \sigma=\operatorname{order} \bar{\eta} i \bar{\eta}^{3}, \quad r=$ $\operatorname{order}\left(R_{1} R_{2} R_{3}\right)^{2}, s=\operatorname{order}\left(R_{3} R_{2} R_{1}\right)^{2}$.

(i) If $3 \nmid \rho \sigma$, then $\Gamma_{\left(t_{13}, F\right)}=\left\{\left\{R_{2},\left(R_{1} R_{2} R_{3}\right)^{2}\right\}\right\}=Z_{p} \times Z_{r}$ and $\Gamma_{\left(t_{31}, F^{\prime}\right)}=\left\{\left\{R_{2},\left(R_{3} R_{2} R_{1}\right)^{2}\right\}\right\}=Z_{p} \times Z_{s}$, and $r=\rho, \sigma=s$.

(ii) (a) If $3 \mid \sigma$ but $3 \nmid \rho$, then

$$
\Gamma_{\left(t_{13}, F^{\prime}\right)}=\left\{\left\{R_{2},\left(R_{1} R_{2} R_{3}\right)^{2}\right\}\right\}=Z_{p} \times Z_{r}, \quad r=\rho
$$

and

$$
\Gamma_{\left(t_{31}, F\right)}=\left\{\left\{R_{2},\left(R_{3} R_{1} R_{2}\right)^{2 \mu} R_{3} R_{1} R_{2} R_{1}\right\}\right\}=Z_{p} \times Z_{3 s}, \sigma=3 s
$$

where $3 \mu+1 \equiv 0(\bmod r)$.

(b) If $3 \mid \rho$ but $3 \nmid \sigma$, then

$$
\Gamma_{\left(t_{31}, F\right)}=\left\{\left\{R_{2},\left(R_{3} R_{2} R_{1}\right)^{2}\right\}\right\}=Z_{p} \times Z_{s}, \quad s=\sigma
$$

and

$$
\Gamma_{\left(t_{13}, F\right)}=\left\{\left\{R_{2},\left(R_{2} R_{1} R_{3}\right)^{2 \nu} R_{2} R_{1} R_{2} R_{3}\right\}\right\}=Z_{p} \times Z_{3 r}, \quad \rho=3 r
$$


where $3 v+1 \equiv 0(\bmod s)$.

(iii) If $3 \mid \rho$ and $3 \mid \sigma$, then

$$
\begin{array}{ll}
\Gamma_{\left(t_{13}, F\right)}=\left\{\left\{R_{2},\left(R_{1} R_{2} R_{3}\right)^{2}\right\}\right\}=Z_{p} \times Z_{r}, & \rho=3 r \\
\Gamma_{\left(t_{31}, F\right)}=\left\{\left\{R_{2},\left(R_{3} R_{2} R_{1}\right)^{2}\right\}\right\}=Z_{p} \times Z_{s}, & \sigma=3 s .
\end{array}
$$

(iv) $\left.\Gamma_{\left(t_{13}, F\right)}\right) \mathscr{F}_{t_{13}, F}$ has 3 elements represented by $F,\left(R_{2} R_{3}\right)^{-1} F$, $\left(R_{1} R_{2}\right) F$ if $\Gamma_{F}=\{1\}$, and has only one element if $\Gamma_{F} \neq\{1\}$.

Proof. From $\S 13$, we have $R_{i} R_{j} t_{k j}=t_{i k}$ for any distinct $i, j, k$. Thus we get the diagrams
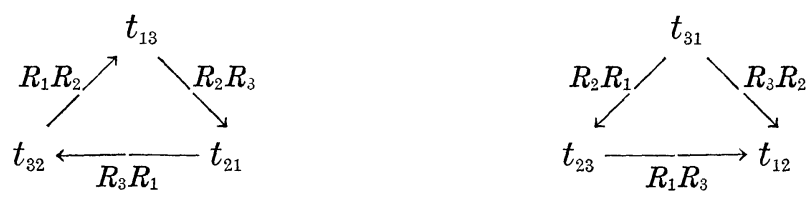

Let $J$ be the element in Aut $F$ permuting $(1,2,3)$ into $(2,3,1)$. From $\S 16$ we know that if $3 \nmid \rho$,

$$
J=\left(R_{1} R_{2} R_{3}\right)^{2 \mu} R_{1} R_{2}=\left(R_{2} R_{3} R_{1}\right)^{2 \mu} R_{2} R_{3}=\left(R_{3} R_{1} R_{2}\right)^{2 \mu} R_{3} R_{1}
$$

where $3 \mu+1 \equiv 0(\bmod \rho)$.

Similarly, if $3 \nmid \sigma$,

$$
J^{-1}=\left(R_{3} R_{2} R_{1}\right)^{2 \nu} R_{3} R_{2}=\left(R_{2} R_{1} R_{3}\right)^{2 \nu} R_{2} R_{1}=\left(R_{3} R_{1} R_{2}\right)^{2 \nu} R_{3} R_{1}
$$

where $3 \nu+1 \equiv 0(\bmod \sigma)$.

For every product of the form $\gamma=\left(R_{1} R_{2}\right)\left(R_{2} R_{3}\right)\left(R_{3} R_{1}\right) \cdots, t_{13} \in \gamma F$; and thus every such $\gamma$ which fixes $t_{13}$ is in $\Gamma_{\left(t_{13}, F\right)}$. A similar assertion holds for any permutation of $(1,2,3)$.

If 3 does not divide $\rho$, we infer from $J R_{2} R_{1}\left(t_{31}\right)=J\left(t_{23}\right)=t_{31}$ that $J R_{2} R_{1} \in \Gamma_{\left(t_{12}, F^{\prime}\right)}$. Moreover, $\left(J R_{2} R_{1}\right)^{3}=\left(\left(R_{3} R_{1} R_{2}\right)^{2 \mu} R_{3} R_{1} R_{2} R_{1}\right)^{3} \cdot=\left(R_{3} R_{1} R_{2}\right)^{8 \mu}$ $\left(R_{3} R_{1} R_{2} R_{1}\right)^{3}$ since by Proposition 18.2.3(i), $R_{3} R_{1} R_{2} R_{1}$ commutes with $\left(R_{3} R_{1} R_{2}\right)^{2}$. As before, set $a=R_{3} R_{1} R_{2} R_{1}, b=R_{1}^{-1} R_{3} R_{1} R_{2}, c=R_{2}^{-1} R_{3} R_{2} R_{1}$. Then $a^{3}=a^{2} \cdot a=a^{2} b c=a b \cdot a c=\left(R_{3} R_{1} R_{2}\right)^{2}\left(R_{3} R_{2} R_{1}\right)^{2}$. Therefore

$$
\left(R_{3} R_{1} R_{2}\right)^{6 \prime \prime}\left(R_{3} R_{1} R_{2} R_{1}\right)^{3}=\left(R_{3} R_{1} R_{2}\right)^{-2} \cdot\left(R_{3} R_{1} R_{2}\right)^{2}\left(R_{3} R_{2} R_{1}\right)^{2}=\left(R_{3} R_{2} R_{1}\right)^{2} .
$$

Similarly, if $3 \nmid \sigma$, then we infer from $J^{-1} R_{2} R_{3}\left(t_{13}\right)=J^{-1}\left(t_{21}\right)=t_{13}$ that $J^{-1} R_{2} R_{3} \in \Gamma_{\left(t_{13}, F\right)}$. Moreover,

$$
\left(J^{-1} R_{2} R_{3}\right)^{3}=\left(R_{2} R_{1} R_{3}\right)^{2 \nu} R_{2} R_{1} R_{2} R_{3} \cdot J^{-2}=\left(R_{2} R_{1} R_{3}\right)^{6 \nu}\left(R_{2} R_{1} R_{2} R_{3}\right)^{3}
$$

since $R_{2} R_{1} R_{2} R_{3}$ commutes with $\left(R_{2} R_{1} R_{3}\right)^{2}$ by the cited result.

As before

$$
\left(R_{2} R_{1} R_{3}\right)^{6 \nu}\left(R_{2} R_{1} R_{2} R_{3}\right)^{3}=\left(R_{2} R_{1} R_{3}\right)^{-2 \nu} \cdot\left(R_{2} R_{1} R_{3}\right)^{2}\left(R_{1} R_{2} R_{3}\right)^{2}=\left(R_{1} R_{2} R_{3}\right)^{2} .
$$


If $3 \nmid \rho$, then $\left\{\left\{J R_{1} R_{2}\right\}\right\}=\left\{\left\{\left(J R_{1} R_{2}\right)^{3}\right\}\right\}=\left\{\left\{\left(R_{3} R_{2} R_{1}\right)^{2}\right\}\right\}$, and similarly, if $3 \nmid \sigma$, then $\left\{\left\{J^{-1} R_{2} R_{3}\right\}\right\}=\left\{\left\{\left(R_{1} R_{2} R_{3}\right)^{2}\right\}\right\}$. Set

$$
\begin{aligned}
\Gamma^{1} & =\left\{\begin{array}{lll}
\left\{\left\{R_{2},\left(R_{2} R_{1} R_{3}\right)^{2 \nu} R_{2} R_{1} R_{2} R_{3}\right\}\right\} & \text { if } & 3 \nmid \sigma \\
\left\{\left\{R_{2},\left(R_{1} R_{2} R_{3}\right)^{2}\right\}\right\} & \text { if } & 3 \mid \sigma
\end{array}\right. \\
\widetilde{\Gamma}^{1} & =\left\{\begin{array}{lll}
\left\{\left\{R_{2},\left(R_{3} R_{1} R_{2}\right)^{2 \mu} R_{3} R_{1} R_{2} R_{1}\right\}\right\} & \text { if } 3 \nmid \rho \\
\left\{\left\{R_{2},\left(R_{3} R_{2} R_{1}\right)^{2}\right\}\right\} & \text { if } & 3 \mid \rho .
\end{array}\right.
\end{aligned}
$$

We have $\Gamma^{1} \subset \Gamma_{\left(t_{13}, F^{\prime}\right)}$ and $\hat{\Gamma}^{1} \subset \Gamma_{\left(t_{31}, F^{\prime}\right)}$. By Proposition 18.2.3(i), $\left(R_{2} R_{1} R_{3}\right)^{2 \nu} R_{2} R_{1} R_{2} R_{3}$ fixes the point $\widetilde{s}_{12}$ as well as $t_{13}$, hence each point of the $C$-line through $\Delta_{123}$, and hence commutes with $R_{2}$. Each element in $\left\{\left\{R_{2}\right\}\right\} \cap\left\{\left\{\left(R_{2} R_{1} R_{3}\right)^{2 \nu} R_{2} R_{1} R_{2} R_{3}\right\}\right\}$ fixes each point of two $C$-lines and therefore is the identity. Hence the order of $\Gamma^{1}$ is po. Similarly, the order of $\widetilde{\Gamma}^{1}=p \sigma$. Thus assertions (i), (ii), (iii) of the proposition is equivalent to: $\Gamma^{1}=\Gamma_{\left(t_{13}, F^{\prime}\right)}$ and $\widetilde{\Gamma}^{1}=\Gamma_{\left(t_{31}, F\right)}$. Making use of the isomorphism $\Gamma(\varphi) \rightarrow \Gamma(\bar{\varphi})$ given by complex conjugation, which sends $t_{31}(\varphi)$ to $t_{13}(\bar{\varphi})$, it suffices to prove only that $\Gamma^{1}=\Gamma_{\left(t_{13}, F\right)}$.

To prove this equality, we apply Proposition 6.6, arguing as in the proof of the preceding proposition. By Lemma 13.4 the 3-faces of $F$ containing $t_{13}$ are

$$
\widetilde{R}_{2}, \widetilde{R}_{2}^{-1}, \widetilde{R}_{2} R_{3},\left(R_{1} \widetilde{R}_{3}\right)^{-1}, R_{2} \widetilde{R}_{3} R_{2},\left(R_{2} \widetilde{R}_{1} R_{3}\right)^{-1} .
$$

Thus the cells in $\mathscr{F}_{t_{13}, F}$ adjacent to $F_{t_{13}}$ are of the form $\gamma F$ with $\gamma$ in the set

$$
F\left[t_{13}\right]: R_{2}^{-1}, R_{2},\left(R_{2} R_{3}\right)^{-1}, R_{1} R_{2},\left(R_{2} R_{3} R_{2}\right)^{-1}, R_{2} R_{1} R_{2} .
$$

First we verify

$$
\mathscr{N}\left(\mathscr{N}\left(F_{t_{13}}\right)\right) \subset \Gamma^{1}\left(\mathscr{N}\left(F_{t_{13}}\right) \cup F_{t_{13}}\right) \text {. }
$$

We must show that for each $\gamma \in F\left[t_{13}\right]$ and $\delta \in F\left[\gamma^{-1} t_{13}\right]$

$$
\gamma \delta \in \Gamma^{1}\left(F\left[t_{13}\right] \cup 1\right) \text {. }
$$

For $\gamma=R_{2}^{+1}$, we have $\gamma \in \Gamma^{1}$ and the assertion is obvious. If $\gamma=$ $\left(R_{2} R_{3}\right)^{-1}, \gamma^{-1} t_{13}=R_{2} R_{3} t_{13}=t_{21}$; we have for

$$
\begin{gathered}
F\left[t_{21}\right]: R_{3}^{-1}, R_{3},\left(R_{3} R_{1}\right)^{-1}, R_{2} R_{3},\left(R_{3} R_{1} R_{3}\right)^{-1}, R_{3} R_{2} R_{3} \\
\begin{aligned}
&\left(R_{2} R_{3}\right)^{-1} F\left[t_{21}\right]: R_{3}^{-1} R_{2}^{-1} R_{3}^{-1}, R_{3}^{-1} R_{2}^{-1} R_{3}, R_{3}^{-1} R_{2}^{-1} R_{1}^{-1} R_{3}^{-1}, 1,\left(R_{3} R_{1} R_{3} R_{2} R_{3}\right)^{-1}, \\
& R_{2} \\
&\left(R_{3} R_{2} R_{3}\right)^{-1} \in F\left[t_{13}\right], R_{3}^{-1} R_{2}^{-1} R_{3}=R_{2} R_{3}^{-1} R_{2}^{-1}=R_{2}\left(R_{2} R_{3}\right)^{-1} \in \Gamma^{1} F\left[t_{13}\right], \\
& R_{3}^{-1} R_{2}^{-1} R_{1}^{-1} R_{3}^{-1}=\left(R_{1} R_{2} R_{3}\right)^{-2} R_{1} R_{2} \in \Gamma^{1} F\left[t_{13}\right],
\end{aligned}
\end{gathered}
$$

and

$$
\left(R_{3} R_{1} R_{3} R_{2} R_{3}\right)^{-1}=\left(R_{3} R_{1} R_{2} R_{3} R_{2}\right)^{-1}=R_{2}^{-1}\left(R_{1} R_{2} R_{3}\right)^{-2} R_{1} R_{2} R_{3} \cdot R_{3}^{-1} \in \Gamma^{1} R_{1} R_{2} .
$$


Thus $\left(R_{2} R_{3}\right)^{-1} F\left[t_{21}\right] \subset \Gamma^{1}\left(F\left[t_{13}\right] \cup 1\right)$. In a similar way we verify the remaining claims in $\left(1^{\prime}\right)$. For example,

$$
\begin{aligned}
& R_{2} R_{1} R_{2} F\left[\left(R_{2} R_{1} R_{2}\right)^{-1} t_{13}\right]=R_{2} R_{1} R_{2} F\left[t_{32}\right] \\
& \quad=R_{2} R_{1} R_{2}\left\{R_{1}^{-1}, R_{1},\left(R_{1} R_{2}\right)^{-1}, R_{3} R_{1},\left(R_{1} R_{2} R_{1}\right)^{-1}, R_{1} R_{3} R_{1}\right\} \\
& \quad=\left\{R_{1} R_{2}, R_{2} R_{1} R_{2} R_{1}, R_{2}, R_{2} R_{1} R_{2} R_{3} R_{1}, 1, R_{2} R_{1} R_{2} R_{1} R_{3} R_{1}\right\} .
\end{aligned}
$$

We have $R_{2} R_{1} R_{2} R_{3} R_{1}=R_{2}\left(R_{1} R_{2} R_{3}\right)^{2}\left(R_{2} R_{3}\right)^{-1}$ and $R_{2} R_{1} R_{2} R_{1} R_{3} R_{1}=$ $R_{2}\left(R_{1} R_{2} R_{3}\right)^{2}\left(R_{2} R_{3}\right)^{-1} R_{3}$, and $R_{3}^{-1} R_{2}^{-1} R_{3}=R_{2} R_{3}^{-1} R_{2}^{-1}$. Therefore

$$
R_{2} R_{1} R_{2} R_{1} R_{3} R_{1}=R_{2}\left(R_{1} R_{2} R_{3}\right)^{2} R_{2} R_{3}^{-1} R_{2}^{-1}=R_{2}^{2}\left(R_{1} R_{2} R_{3}\right)^{2}\left(R_{2} R_{3}\right)^{-1} .
$$

In this way we see that $R_{2} R_{1} R_{2} F\left[\left(R_{2} R_{1} R_{2}\right)^{-1} t_{13}\right] \subset \Gamma^{1}\left(F\left[t_{13}\right] \cup 1\right)$. So much for the proof of (1).

From Proposition 6.6, it follows that

$$
\Gamma_{\left(t_{13}, F\right)}=\Gamma^{1} S
$$

where $S=\left\{\gamma \in\left(F\left[t_{13}\right] \cup\{1\}\right) \Gamma_{F} ; \gamma t_{13}=t_{13}\right\}$. Set $T^{1}=F\left[t_{13}\right] \cup\{1\}$. For any subset $S^{1} \subset T^{1}$ such that $\Gamma^{1} T^{1}=\Gamma^{1} S^{1}$, we have $\Gamma^{1}\left(S^{1} \Gamma_{F^{1}} \cap \Gamma_{\left(t_{13}, F\right)}\right)=$ $\Gamma_{\left(t_{13}, F\right)}$ by Remark 1 following Proposition 6.6. The images of $t_{13}$ under $F\left[t_{13}\right] \Gamma_{F}$ are seen from

$$
R_{2} t_{13}=t_{13},\left(R_{2} R_{3}\right)^{-1} t_{21}=t_{13},\left(R_{1} R_{2}\right) t_{32}=t_{13}, R_{1} R_{2} R_{1} t_{32}=t_{13} .
$$

Inasmuch as $\Gamma^{1}\left(R_{2} R_{3}\right)^{-1}$ contains $\left(R_{2} R_{3} R_{2}\right)^{-1}$, we can ignore the contribution of $\left(R_{2} R_{3} R_{2}\right)^{-1}$. That is, set $S^{1}=\left\{1,\left(R_{2} R_{3}\right)^{-1}, R_{1} R_{2}\right\}$. Then

$$
\begin{aligned}
\Gamma^{1} S^{1} & =\Gamma^{1}\left(\{1\} \cup F\left[t_{13}\right]\right), \\
t_{13} & =\left(R_{2} R_{3}\right)^{-1} J t_{13}=\left(R_{1} R_{2}\right) J^{i 1} t_{13} .
\end{aligned}
$$

Consequently

$$
\Gamma_{\left(t_{13}, F\right)}=\left\{\begin{array}{l}
\Gamma^{1}\left\{1,\left(R_{2} R_{3}\right)^{-1} J,\left(R_{1} R_{2}\right) J^{-1}\right\} \quad \text { if } \quad J \in \Gamma_{F} \\
\Gamma^{1} \text { if } J \notin \Gamma_{F} .
\end{array}\right.
$$

All the assertions of the proposition follow from $\left(^{* *}\right)$ and $\left(^{*}\right)$.

REMARK 18.2.5. The number of polyhedra in the polyhedral space $Y\left(t_{13}, \mathscr{F}\right)$ surrounding $t_{13}$ is

$$
\# \mathscr{F}_{t_{13}, F}=p \rho \text {. }
$$

Similarly,

$$
\# \mathscr{F}_{t_{31}, F}=p \sigma \text {. }
$$

From Proposition 18.2.3, we have

$$
\# \mathscr{F}_{s_{i j}, F}=\# \mathscr{F}_{s_{i j}, F}=2 \rho \sigma \text {. }
$$


Proposition 18.2.6. The stabilizer in $\Gamma$ of $e \in E_{k}(F)(k=1,2,3)$ is given by the following lists

\begin{tabular}{|c|c|c|}
\hline$\eta(e, F)$ & $\Gamma_{(\boldsymbol{c}, F)}$ & order \\
\hline$p_{12} t_{32}$ or $p_{12} t_{23}$ & $\left\{\left\{R_{1}\right\}\right\}$ & $p$ \\
\hline$p_{12} s_{12}\left(v i a s_{12}^{*}\right.$ or $\left.s_{21}^{*}\right)$ & $\{1\}$ & 1 \\
\hline & $\left\{\left\{\left\{J^{-1} R_{1} R_{2}\right\}\right\}\right.$ & $\int \rho$ if $J \in \Gamma$ \\
\hline$s_{12} \iota_{32}$ & $\left\{\left\{\left\{\left(R_{3} R_{1} R_{2}\right)^{2}\right\}\right\}\right.$ & $\left\{\begin{array}{l}r \\
\text { if } J \notin \Gamma\end{array}\right.$ \\
\hline ct & $\int\left\{\left\{J R_{1} R_{3}\right\}\right\}$ & $\int \sigma$ if $J \in \Gamma$ \\
\hline$s_{31} \iota_{23}$ & $\left\{\left\{\left\{\left(R_{2} R_{1} R_{3}\right)^{2}\right\}\right\}\right.$ & $\{s$ if $J \notin \Gamma$ \\
\hline$\widetilde{s}_{13} s_{12}$ & $\left\{\begin{array}{l}\left\{\left\{J^{-1} R_{1} R_{2}, R_{3} R_{1} R_{2}\right\}\right\} \\
\left\{\left\{R_{3} R_{1} R_{2}\right\}\right\}\end{array}\right.$ & $\begin{cases}2 \rho & \text { if } J \in \Gamma \\
2 r & \text { if } J \notin \Gamma\end{cases}$ \\
\hline$\widetilde{s}_{21} s_{31}$ & $\left\{\left\{R_{2} R_{1} R_{3}\right\}\right\}$ & $2 s$ \\
\hline$e_{1}^{\vdash} \cap F$ & $\left\{\left\{R_{1}\right\}\right\}$ & $p$ \\
\hline$\Delta_{123}$ & $\left\{\begin{array}{l}J^{-1} R_{1} R_{2} \\
\left\{\left\{\left(R_{1} R_{2} R_{3}\right)^{2}\right\}\right\}\end{array}\right.$ & $\begin{array}{l}\rho \text { if } J \in \Gamma \\
r \text { if } J \notin \Gamma\end{array}$ \\
\hline$\Delta_{321}$ & $\left\{\begin{array}{l}J R_{1} R_{3} \\
\left\{\left\{\left(R_{3} R_{2} R_{1}\right)^{2}\right\}\right\}\end{array}\right.$ & $\begin{cases}\sigma & \text { if } J \in \Gamma \\
s \text { if } J \notin \Gamma\end{cases}$ \\
\hline $\begin{array}{l}\widetilde{R_{1}} \cap \widetilde{R_{1} R_{2}}, \widetilde{R_{2}} \cap \widetilde{R_{1} R} \\
\widetilde{R_{1} R_{2}} \cap R_{1}{\widetilde{R_{2}}}_{2}, \widetilde{R_{2} R_{1}} \\
\widetilde{R_{1}}, \widetilde{R_{1} R_{2}}, \widetilde{R_{2} R_{1}}, R_{1} \widetilde{R}_{2}\end{array}$ & $\begin{array}{l}\cap R_{1} \widetilde{R}_{2} R_{1}, \widetilde{R}_{2} \cap R_{1} \widetilde{R}_{2} R_{1} \\
R_{1} \widetilde{R}_{2} R_{1}\end{array}$ & $\begin{array}{l}1 \\
1 \\
1\end{array}$ \\
\hline
\end{tabular}

Proof. One verifies that $R_{3} R_{1} R_{2} \widetilde{s}_{13}=s_{12}$ and $R_{3} R_{1} R_{2} s_{12}=\widetilde{s}_{13}$. Thus the stabilizer of the 1-face $\widetilde{s}_{13} s_{12}$ contains the cyclic group $\left\{\left\{R_{3} R_{1} R_{2}\right\}\right\}$. Similar for $\widetilde{s}_{21} s_{31}$. By reasoning as in the preceding propositions, one proves the asserted results for $\widetilde{s}_{13} s_{12}$ and $\widetilde{s}_{21} s_{31}$. For $\widetilde{R}_{1} \cap \widetilde{R}_{1} R_{2}$, the stabilizer leaves fixed each of its vertices $p_{12}, \widetilde{s}_{32}, s_{12}$ and its stabilizer is in the intersection of the stabilizer of its vertices; hence the stabilizer fixes each point of the smallest geodesic subspace containing the faces.

\subsection{Riemannian manifold structure on $Y$.}

Assume that $\left|\arg \varphi^{3}\right|<\pi / 2-\pi / p$ and that $(1 / \pi) \arg \varphi^{3} \in \boldsymbol{Q}$. Then the space $Y(\varphi)$ has been defined as well as the canonical map $\pi$ of $Y$ onto $\mathrm{Ch}^{2}$. At any point of $Y$ which is not in the $\Gamma$ orbit of a point in one of the six $\Delta_{i j h}$, the map $\pi$ is a local homeomorphism. At faces lying in some $\Delta_{i j h}$, the neighborhood of the faces are given by the results in $\S 18.2$. The hypotheses of Proposition 6.4.1 are satisfied by the abutted family $\Gamma \Omega$. Indeed one can give $Y$ a complex analytic structure so that $\pi: Y \rightarrow C h^{2}$ is holomorphic (cf. Proposition 6.4.2). Thus we conclude (cf. $\S 19$ for details). 
Lemma 18.3. For $\left|\arg \varphi^{3}\right|<\pi / 2-\pi / p$ and $(1 / \pi) \arg \varphi^{3} \in \boldsymbol{Q}$, the space $Y(\varphi)$ has the structure of a Riemannian manifold such that $\pi: Y \rightarrow C h^{2}$ is differentiable.

The infinitesimal Riemannian metric in $C h^{2}$ pulls back to a metric on $Y$ which may be degenerate at points at which $\pi$ is not a local homeomorphism. However, it is not difficult to define a Riemannian metric on $Y(\varphi)$ that is preserved by $\Gamma$. For example, let $\Gamma_{0}$ be a torsion-free normal subgroup of $\Gamma$ of finite index - such a subgroup exists in any finitely generated metric group by a result of Selberg. Then choose a Riemannian metric on the manifold $\Gamma_{0} \backslash Y$. Averaging over the finite group $\Gamma / \Gamma_{0}$, we can assume that the metric $d$ on $\Gamma_{0} \mid Y$ is $\Gamma$-stable. The pull-back of $d$ to $Y$ gives a $\Gamma$-stable metric on $Y$.

\section{4. $\operatorname{Aut}_{\Gamma} \Omega,\left|\arg \varphi^{3}\right|<\pi / 2-\pi / p, 3 \mid \operatorname{gcd}(\rho, \sigma)$.}

We take up the question of the order of $\operatorname{Aut}_{\Gamma} \Omega$ that was mentioned in Remark 3 of $\S 16$.

Lemma 18.5. Assume $\left|\arg \varphi^{3}\right|<\pi / 2-\pi / p$. Let $\rho=\operatorname{order} \bar{\eta} i \varphi^{3}$, $\sigma=\operatorname{order} \bar{\eta} i \bar{\varphi}^{3}$ and assume that $3 \mid \operatorname{gcd}(\rho, \sigma)$. Then $\# \operatorname{Aut}_{\Gamma} \Omega=1$.

Proof. Let $Y$ denote the manifold $Y(\varphi)$, let $Y^{*}$ denote the simply connected covering space of $Y$, and let $\Gamma^{*}$ denote the lift of $\Gamma$ to $Y$. Then $\Gamma^{*} / \pi_{1}(Y)=\Gamma$ and $\Gamma^{*}$ operates discontinuously as a group of isometries on the Riemannian manifold $Y^{*}$. Let $\Omega^{*}$ denote a lift of the polyhedron $\Omega$; inasmuch as $\Omega$ is a topological cell (by Lemma 3.3.2) $\Omega^{*}$ is a cell mapping homeomorphically onto $\Omega$. The space $Y^{*}$ is the joined $\Gamma^{*}$-space of the abutted family of polyhedron $\Gamma^{*} \Omega^{*}$. The group $\Gamma^{*}$ operates discontinuously on $Y^{*}$ and satisfies both (CD1) and (CD2) of Theorem 6.3.3. It follows that $\mathrm{Aut}_{\Gamma^{\circ}} \Omega^{*}$ is generated by $R_{2}^{*}$, the set of words corresponding to shortest circuits in $Y^{*}$ around 2 -faces of $\Omega^{*}$. From this in turn it follows that $\operatorname{Aut}_{\Gamma} \Omega$ is generated by $\mathscr{R}_{2}$, the set of words corresponding to shortest circuits in $Y$ around 2 -faces of $\Omega$. Inasmuch as the circuits around 2-faces of $\Omega$ which do not contain an apex correspond to trivial words, we need only consider the circuits around $\Delta_{i j h}$ for permutations ( $\left.i j h\right)$ of (123). By symmetry, it suffices to consider only circuits around $\Delta_{123}$.

Given such a circuit $\Omega, R_{1} R_{2}, R_{1} R_{2} \cdot R_{3} R_{1} \Omega, R_{1} R_{2} \cdot R_{3} R_{1} \cdot R_{2} R_{3} \Omega, \cdots$ with $R_{1} R_{2} \cdot R_{3} R_{1} \cdots \Omega=\Omega$, then set $\gamma=R_{1} R_{2} \cdot R_{3} R_{1} \cdots$. We have $\gamma \in \operatorname{Aut}_{\Gamma} \Omega$. We can assume that $\varphi \neq 1$, otherwise $3 \mid \rho$ and $3 \mid \sigma$, imply $p=3$ and $\Omega(\varphi)$ satisfies $\left(\mathrm{CD}_{2}\right)$; in that case the lemma is known (cf. Remark 2 of $\S 16)$. Hence $\operatorname{Aut}_{\Gamma} \Omega=Z_{3}$ and $\gamma=1, J$, or $J^{-1}$. Thus $\gamma$ sends into $\Delta_{123}$ either $\Delta_{123}, \Delta_{312}$, or $\Delta_{231}$. Accordingly, $\gamma$ has the form 


$$
\left(R_{1} R_{2} R_{3}\right)^{2 m},\left(R_{1} R_{2} R_{3}\right)^{2 m} R_{1} R_{2},\left(R_{1} R_{2} R_{3}\right)^{2 m} R_{1} R_{2} R_{3} R_{1} \text {. }
$$

We wish to prove $\gamma=1$. Hence we need only dismiss the possibilities $\gamma=\left(R_{1} R_{2} R_{3}\right)^{2 m} R_{1} R_{2}$ or $\left(R_{1} R_{2} R_{3}\right)^{2 m} R_{1} R_{2} R_{3} R_{1}$.

Consider the canonical map $\pi: Y \rightarrow C h^{2}$. The circuit in $C h^{2}$ corresponding to $\gamma$ places side by side $3 m+1$ (resp. $3 m+2)$ images of $\Omega$ bounded by spinal surfaces meeting in a common slice containing $\Delta_{123}$. Each of the $3 m+1$ (resp. $\left.3 m+2\right)$ angles formed equals $\Varangle\left(R_{1} \hat{R}_{2}\right)^{-1}$, $R_{2} R_{3}=\arg \bar{\eta} i \varphi^{3}$ (cf. $\S 15$ ). Hence $\left(\bar{\eta} i \varphi^{3}\right)^{3 m+\varepsilon}=1 \varepsilon=1$ or 2 . Consequently, $\rho \mid 3 m+\varepsilon(\varepsilon=1,2)$. This contradicts $3 \mid \rho$. It follows that $\operatorname{Aut}_{\Gamma} \Omega=1$.

19. Complex analytic structure on $Y(\varphi),\left|\arg \varphi^{3}\right|<\pi / 2-\pi / p$, $\pi^{-1} \arg \varphi \in \boldsymbol{Q}$.

We continue the notation of $\S 18$, writing $\Gamma=\Gamma(\varphi), \Delta=\Delta(\varphi)$, $F=\Omega(\varphi), Y=Y(\varphi), \rho=$ order $\bar{\eta} i \varphi^{3}, \sigma=\operatorname{order} \bar{\eta} i \bar{\varphi}^{3}$. Assume that $\rho$ and $\sigma$ are finite. Then

$$
\rho\left(\frac{\pi}{2}-\frac{\pi}{p}+\arg \varphi^{3}\right)=2 m \pi, \sigma\left(\frac{\pi}{2} \frac{\pi}{p}-\arg \varphi^{3}\right)=2 n \sigma
$$

where $\operatorname{gcd}(m, \rho)=1=\operatorname{gcd}(n, \sigma)$. Set

$$
\xi=\exp (2 \pi i / \rho), \quad \xi=\exp (2 \pi i / \sigma) .
$$

Then $\xi^{m}=\bar{\eta} i \varphi^{3}, \zeta^{n}=\bar{\eta} i \bar{\varphi}^{3}$.

The canonical map $\pi$ of $Y$ onto the ball $B$ is clearly a homeomorphism in the neighborhood of any point $p$ of thecell $\eta(\gamma, F)$ of $Y$ if $p$ does not lie on $\eta\left(\gamma, \Delta_{i j k}\right)(i, j, k$ any permutation of $1,2,3)$; in the neighborhood of such $p$, one chooses as coordinates the pull-back of a standard coordinate system on the ball $B$.

We next describe the choice of a coordinate system in the neighborhood of a point in $\eta\left(\gamma, \Delta_{i j k}\right)$. By symmetry, we can take $\gamma=1$ and $p \in \eta\left(1, \Delta_{312}\right)$. We shall cover a neighborhood of $\eta\left(1, \Delta_{312}\right)$ in $Y$ by pull-backs of three balls in $B$ centered at the vertices of $\Delta_{312}$.

We first consider the case $p=s_{12}$. Recall that (cf. (12.1)) $s_{12}=$ $I_{1} \cap I_{2}^{\prime}$ and that the $C$-lines $I_{1}, I_{2}^{\perp}$ are orthogonal at $s_{12}$ (cf. Remark 2 following Lemma 12.3). Therefore we can choose a standard nonhomogeneous coordinate system centered at $s_{12}$ with $u=0$ on $I_{2}^{\prime}$ and $v=0$ on $I_{1}$. Since $u$ and $v$ are unique up to a scalar factor of modulus one, (each line has a unique holomorphic structure induced from $C^{3}$ !) we can make the choice unique by the additional conditions $u\left(\widetilde{s}_{13}\right)>0, v\left(\widetilde{s}_{32}\right)>0$. Let $\alpha$ be a positive number such that the ball $B_{\alpha}\left(s_{12}\right)$ with center at $s_{12}$ and radius $d$ meet no face of $F$ other than those containing $s_{12}$. Let $C_{12}$ denote the subset of $B_{\alpha} \times C \times C$ defined by 


$$
C_{12}=\left\{(x, \widetilde{u}, \widetilde{v}) ; \widetilde{u}^{m}=u(x), \widetilde{v}^{n}=v(x)\right\} .
$$

Let $\psi: C_{12} \rightarrow B_{\alpha}\left(s_{12}\right)$ denote the projection on the first factor. We can define an operation of $\Gamma_{\left(s_{12}, F\right)}$ on $C_{12}$ so that $\psi$ is a $\Gamma_{\left(s_{12}, F\right)}$ map. We see this as follows.

By (9.1.6) the eigenvalue of $R_{2} R_{1} R_{2} R_{3}$ corresponding to its fixed point $s$ in $B$ is $-\eta^{2}$ and on standard nonhomogeneous coordinates centered at $s$ its eigenvalues are $\left(\eta^{3} i \varphi^{3} /-\eta^{2}, \eta^{3} i \bar{\varphi}^{3}\left(-\eta^{2}\right)\right)$; that is, $\left(\bar{\zeta}^{n}, \bar{\xi}^{m}\right)$. The same is true for $R_{3} R_{2} R_{1} R_{2}$ (for it has the same characteristic polynomial) which fixes $s_{12}$. Hence

$$
\begin{aligned}
& u\left(R_{3} R_{2} R_{1} R_{2} x\right)=\bar{\zeta}^{n} u(x) \\
& v\left(R_{3} R_{2} R_{1} R_{2} x\right)=\bar{\xi}^{m} v(x)
\end{aligned}
$$

for all $x \in B$. Similarly by (9.1.1) and (9.1.2)

$$
\begin{aligned}
& u\left(\left(R_{3} R_{1} R_{2}\right)^{2} x\right)=u(x), v\left(\left(R_{3} R_{1} R_{2}\right)^{2} x\right)=\bar{\xi}^{m} v(x) \\
& u\left(\left(R_{3} R_{2} R_{1}\right)^{2} x\right)=\bar{\zeta}^{n} u(x), v\left(\left(R_{3} R_{2} R_{1}\right)^{2} x\right)=v(x)
\end{aligned}
$$

for all $x \in B$.

Thus we define for all $y=(x, \widetilde{u}, \widetilde{v}) \in C_{12}$,

$$
\begin{aligned}
& \widetilde{u}\left(R_{3} R_{2} R_{1} R_{2} y\right)=\bar{\zeta} \widetilde{u}(y), \quad \widetilde{v}\left(R_{3} R_{2} R_{1} R_{2} y\right)=\bar{\xi} \widetilde{v}(y) \\
& \widetilde{u}\left(\left(R_{3} R_{1} R_{2}\right)^{2} y\right)=\widetilde{u}(y), \quad \widetilde{v}\left(\left(R_{3} R_{1} R_{2}\right)^{2} y\right)=\bar{\xi} \widetilde{v}(y) \\
& \widetilde{u}\left(\left(R_{3} R_{2} R_{1}\right)^{2} y\right)=\bar{\zeta} \widetilde{u}(y), \quad \widetilde{v}\left(\left(R_{3} R_{2} R_{1}\right)^{2} y\right)=\widetilde{v}(y) .
\end{aligned}
$$

Holomorphically, take $C_{12} \approx\left\{(\tilde{u}, \widetilde{v}) \in C^{2} ;|\tilde{u}|^{2 m}+|\widetilde{v}|^{2 n}<r^{2}\right\}$. It is clear now that $\psi: C_{12} \rightarrow B_{\alpha}\left(s_{12}\right)$ is a $\Gamma_{\left(s_{12}, F\right)}$ map and also holomorphic. The canonical map $\pi: y_{s_{12}, F} \rightarrow B$ branches over $B_{r}\left(s_{12}\right)$ in exactly the same way that $C_{12}$ does. Hence the projection $\psi: C_{12} \rightarrow B$ factors through $Y_{s_{12}, F}$ and there is a unique $\Gamma_{\left(s_{12}, F\right)}$ homeomorphism $f_{12}: C_{12} \rightarrow Y_{s_{12}, F} \cap$ $\pi^{-1}\left(B_{\alpha}\right)$ such that $\pi \circ f_{12}=\psi, \tilde{u}>0$ on $f_{12}^{-1}\left(\eta\left(\left[s_{12}, \widetilde{s}_{13}\right], F\right)\right)$, and $\widetilde{v}>0$ on $f_{12}^{-1}\left(\left(\eta\left[s_{12}, \widetilde{s}_{13}\right], F\right)\right)$. We sometimes denote $f_{12}$ as $f_{s_{12}}$.

In exactly the same way, one defines a space $C_{i j}$ (resp. $C_{\tilde{i} j}$ ) for each vertex $s_{i j}$ (resp. $\left.s_{i_{j}}\right), i \neq j, i, j=1,2,3$; this yields six space. Given any $\gamma \in G\left(s_{12}, F\right)$ (cf. $\S 6.6$ for definition), $\bar{\gamma}^{-1} s_{12} \in \mathscr{S}=\left\{s_{i j}, s_{i j}\right.$; $i \neq j, i, j=1,2,3\}$, and for each $s \in \mathscr{S}$ we get a commutative diagram

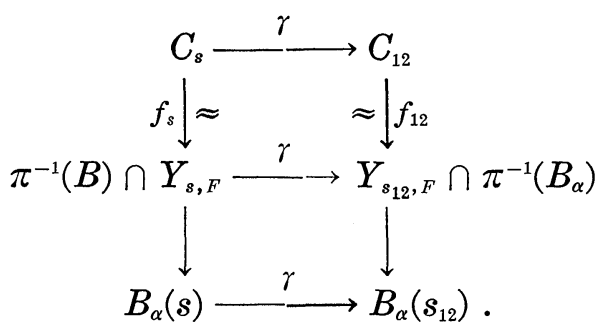

We next prove that:

$\gamma: C_{s} \longrightarrow C_{12}$ is holomorphic for all $\gamma \in G\left(s_{12}, F\right)$ where $s=\gamma^{-1} s_{12}$. 
Inasmuch as $f_{s}$ is a $\Gamma_{(s, F)}$ map, it suffices to prove that $\gamma$ is holomorphic for a set of representatives of $\Gamma_{\left(s_{12}, F\right)} G\left(s_{12}, F\right)$; by Proposition 18.2.3(iii), $\left\{1, R_{1}^{-1}, R_{2}^{-1}, R_{3}, R_{3} R_{1}, R_{3} R_{2}\right\}$ is a set of representatives. We shall give the argument only for the cases $\gamma=R_{3}, \gamma=R_{1}^{-1}$ and $\gamma$ $R_{3} R_{1}$, the other cases following by symmetry.

Returning to Figure 14.1, we see that

$$
R_{3}\left[\widetilde{s}_{21}, s_{23}\right]=\left[s_{12}, \widetilde{s}_{13}\right] .
$$

Thus $R_{3} I_{2}=I_{1}$, and in the $C$-line $I_{1}$ the geodesic triangle $R_{3} \Delta_{123}$ abuts $\Delta_{312}$ along the geodesic line segment $\left[s_{12}, \widetilde{s}_{13}\right]$. Similarly

$$
R_{3}\left[\widetilde{s}_{21}, s_{31}\right]=\left[s_{12}, \widetilde{s}_{32}\right], \quad R_{3} I_{1}^{\prime}=I_{2}^{\prime}
$$

and the geodesic triangle $R_{3} \Delta_{213}$ abuts $\Delta_{321}$ along the geodesic line segment $\left[s_{12}, \widetilde{s}_{32}\right]$. Inasmuch as $R_{3}$ is an isometry of the ball, it carries any standard nonhomogeneous coordinate on the $C$-line $I_{2}$ (resp. $I_{1}^{\prime}$ ) centered at $\widetilde{s}_{21}$ to a standard nonhomogeneous coordinate on the $\boldsymbol{C}$ line $I_{1}$ (resp. $I_{2}^{\prime}$ ) centered at $s_{12}$. We next compute the transformation of the coordinates of $C_{21}$ induced by $R_{3}$.

By definition $C_{21}$ has coordinates $\widetilde{u}, \widetilde{v}$; $\widetilde{u}^{m}\left(\right.$ resp. $\left.\widetilde{v}^{n}\right)$ is the unique standard nonhomogeneous coordinates on $I_{2}$ (resp. $I_{1}^{\prime}$ ) centered at $\widetilde{s}_{21}$ with $\tilde{u}>0$ on $f_{21}^{-1}\left(\eta\left(\left[\widetilde{s}_{21}, s_{23}\right], F\right)\right)($ resp. $\widetilde{v}>0)$ on $f_{21}^{-1}\left(\eta\left(\left[\widetilde{s}_{21}, s_{31}\right], F\right)\right)$. By (19.3), $R_{3}$ sends the defining data of the $\widetilde{u}, \widetilde{v}$ coordinates of $C_{21}$ to the defining data for the $\widetilde{u}, \widetilde{v}$ coordinates of $C_{12}$. Consequently, the map $R_{3} C_{\widetilde{21}} \rightarrow C_{12}$ is the map $(\widetilde{u}, \widetilde{v}) \rightarrow(\widetilde{u}, \widetilde{v})$. Thus $R_{3}$ is holomorphic.

Consider next the map $R_{1}^{-1}: C_{13} \rightarrow C_{12}$. We have (cf. (13.1))

$$
\begin{aligned}
& R_{1}^{-1}\left[\widetilde{s}_{13}, t_{32}\right]=\left[s_{12}, t_{32}\right] \\
& R_{1}^{-1}\left[\widetilde{s}_{13}, s_{23}\right]=\left[s_{12}, \widetilde{s}_{32}\right] .
\end{aligned}
$$

The second relation implies that $R_{1}^{-1}$ sends the $\widetilde{v}$ coordinates of $C_{\tilde{13}}$ to the $\widetilde{v}$ coordinates of $C_{12}$, by the same argument that was used above. The first relation yields the diagram

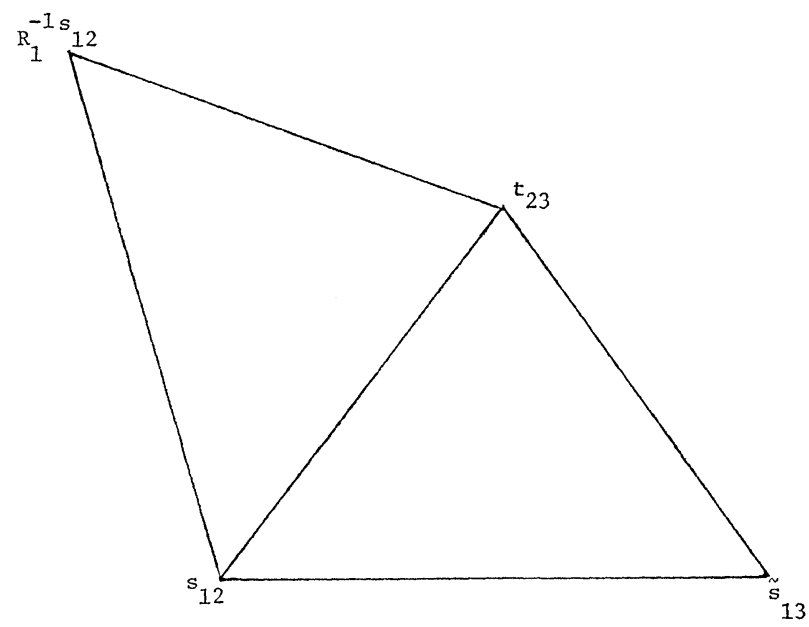


This shows that the image $R_{1}^{-1}\left[\widetilde{s}_{13}, s_{23}\right]$ is the geodesic line segment from $s_{12}$ to $R_{1}^{-1} s_{12}$, which forms with $\left[s_{12}, \widetilde{s}_{13}\right]$ an angle equal to $2 \Varangle$ $t_{32} s_{12} \widetilde{s}_{13}=\pi / 2-\pi / p-\arg \varphi^{3}$ by Lemma 15.2. Thus transformation of coordinates induced by $R_{1}^{-1}: C_{\tilde{13}} \rightarrow C_{12}$ is

$$
(\widetilde{u}, \widetilde{v}) \longrightarrow(\zeta \tilde{u}, \widetilde{v}) \text {; }
$$

it too is holomorphic.

Finally, consider the map $R_{3} R_{1}: C_{s} \rightarrow C_{2}$ where $s=\left(R_{3} R_{1}\right)^{-1} s_{12}=s_{31}$ by (13.1)

$$
\begin{aligned}
& R_{3} R_{1}\left[s_{31}, \widetilde{s}_{32}\right]=\left[s_{12}, \widetilde{s}_{13}\right] \\
& R_{3} R_{1}\left[s_{31}, t_{23}\right]=\left[s_{12}, R_{3} t_{23}\right] .
\end{aligned}
$$

The first relation implies by the argument used above that $R_{3} R_{1}$ transforms the $\tilde{u}$ coordinate of $C_{31}$ to the $\tilde{u}$ coodinate of $C_{12}$. The implication of the second relation becomes clear upon observing that

$$
\begin{aligned}
& R_{3} t_{23}=R_{3}\left(R_{2} R_{1} t_{31}\right) \quad \text { by Lemma 13.3(i) } \\
& R_{3} R_{1} t_{23}=\left(R_{3} R_{2} R_{1}\right) t_{31}
\end{aligned}
$$

and $\left.R_{3} R_{2} R_{1} s_{12}, \widetilde{s}_{32}\right]=\left[\widetilde{s}_{32}, s_{12}\right]$. Consequently $R_{3} R_{2} R_{1}$ rotates $\Delta_{321}$ about the midpoint of $\left[s_{12}, \widetilde{s}_{32}\right] 180^{\circ}$ and the image of $\Delta_{213}$ under $R_{3} R_{1}$ is given (cf. the diagram)

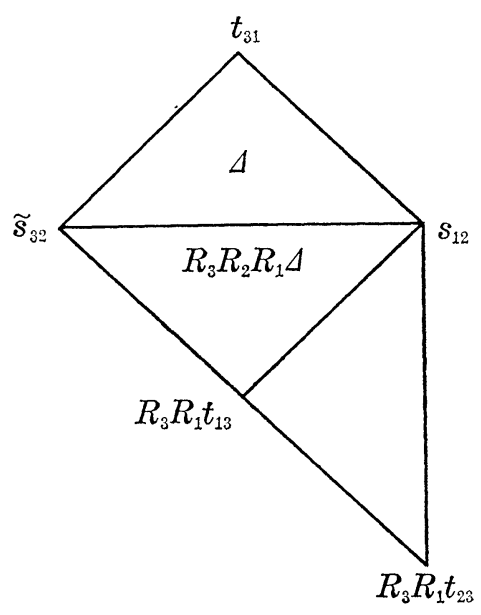

by rotating $\left[s_{12}, \widetilde{s}_{32}\right]$ towards the direction of $\operatorname{Im} u<0$ through an angle $2 \Varangle t_{31} s_{12} \widetilde{s}_{32}$; that is $-\left(\pi / 2-\pi / p+\arg \varphi^{3}\right)$ by Lemma 15.2. Thus the transformation of coordinates induced by $R_{3} R_{1}: C_{31} \rightarrow C_{12}$ is

$$
(\widetilde{u}, \widetilde{v}) \longrightarrow(\widetilde{u}, \bar{\xi} \widetilde{v})
$$

which is holomorphic. 
Analogously, we can consider balls $B_{\beta}\left(t_{32}\right)$ centered at $t_{32}$ of radius $\beta$ and meeting no edge of $\Delta_{312}$ other than those containing $t_{32}$. We can select $\alpha$ and $\beta$ so that

$$
\Delta_{312} \subset B_{\alpha}\left(s_{12}\right) \cup B_{\alpha}\left(\widetilde{s}_{13}\right) \cup B_{\beta}\left(t_{32}\right) .
$$

Define for any integer $i \bmod 3$,

$$
C_{i}=\left\{(x, \widetilde{w}) ; x \in B_{\beta}\left(t_{i}\right), \widetilde{w} \in C ; \widetilde{w}^{m}=w(x)\right\}
$$

where $t_{i}=t_{i-1, i+1}, w$ is the unique standard nonhomogeneous coordinates on $I_{i}$ centered at $t_{i}$ and positive on the geodesic lin segment $\left[t_{i} s_{i i+1}\right]$. Then define the homeomorphism

$$
f_{i}: C_{i} \stackrel{\approx}{\longrightarrow} Y_{t_{i}, F} \cap \pi^{-1}\left(B_{\beta}\left(t_{i}\right)\right)
$$

with properties analogous to (19.1). By the same type of reasoning as above, one proves:

(19.4) For all $\gamma \in G\left(t_{i}, F\right)$ the map induced by $\gamma$ from $C_{j}$ to $C_{i}$ is holomorphic, where $\gamma^{-1} t_{i}=t_{j}(i=1,2,3)$.

The coordinate system on $y$ that we have selected above has the property:

(19.5) For any two overlapping coordinate neighborhoods, the coordinates are biholomorphically related.

The proof is quite simple. For by choice of coordinates, the intersection of two coordinate neighborhoods either

(i) projects by $\pi$ biholomorphically onto a neighborhood in the ball; or

(ii) has one of the forms

(a) $Y_{s, F} \cap Y_{s^{\prime}, F} \cap \pi^{-1}\left(B_{\alpha}(s) \cap B_{\alpha}(\widetilde{s})\right), s, s^{\prime} \in \Delta_{i j k}$

(b) $Y_{s, F} \cap Y_{t, F} \cap \pi^{-1}\left(B_{\alpha}(s) \cap B_{\beta}(t)\right), s, t \in \Delta_{i j k}$.

In Case (i), assertion (19.5) is obvious. In Case ii(a) the two coordinate systems are related by $R_{i} R_{j} R_{k}$ which belongs to $G(s, F)$. In case $s=s_{12}$, this is assured by (19.2); for any other $s$, the analogue of (19.2) is valid by symmetry. Thus it remains only to consider Case (iib). By symmetry, we may take $s=s_{12}, t=t_{32}$. In the overlap (iib), the coordinate function $\widetilde{v}$ of $C_{12}$ has no branch locus on $B_{\beta}(t)$. Moreover, the $\widetilde{u}^{m}$ of $C_{12}$ and $\widetilde{w}^{m}$ of $C_{1}$ are related by the fractional linear transformation of the Poincare disc $I_{1}$ which relates the two standard nonhomogeneous coordinates centered at $s_{12}$ and $t_{32}$ respectively. Hence

$$
\widetilde{u}=\sqrt[m]{\frac{a \widetilde{w}^{m}+b}{b \widetilde{w}^{m}+\bar{a}}}
$$


where neither numerator nor denominator vanish on $B_{\alpha}\left(s_{12}\right) \cap B_{\beta}(t)$. From this assertion (19.5) follows.

From (19.2) and (19.4) we can conclude that the group $\Gamma$ acts holomorphically on $Y$. We summarize our conclusions in the following theorem.

THEOREM 19.1. Let $\phi$ be any complex number of modulus 1 with $\left|\arg \varphi^{3}\right|<\pi / 2-\pi / p$ and $(1 / \pi) \arg \varphi$ a rational number. Let $\Gamma(\varphi)$ denote the group generated by $\mathrm{C}$-reflections with Coxeter diagram

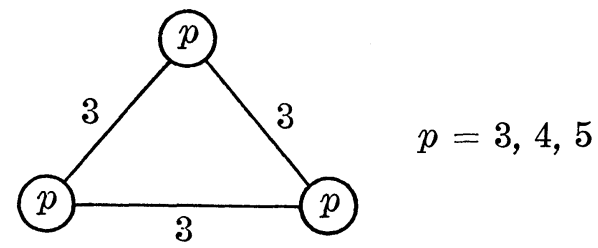

and phase shift $\varphi_{12}=\varphi_{23}=\varphi_{31}=\varphi$. Let $Y(\varphi)$ denote the joined $\Gamma(\varphi)$-space on which $\Gamma(\varphi)$ operates discontinuously (cf. $\S 6.5)$, and $\pi: Y(\varphi) \rightarrow C h^{2}$ the canonical $\Gamma(\varphi)$-map of $Y(\varphi)$ to the ball. Then $Y(\varphi)$ has the structure of a complex analytic manifold satisfying

(1) $\pi$ is holomorphic.

(2) Each $\gamma \in \Gamma(\varphi)$ acts biholomorphically on $Y(\varphi)$.

REMARK 1. If $\pi / 2-\pi / p<\arg \varphi^{3}<3(\pi / 2-\pi / p)$ and $(1 / \pi) \arg \varphi \epsilon$ $Q$, the abutted smooth family of polyhedra $\Gamma \Omega(\varphi)$ satisfies conditions BR of $\S 6.4$ but not hypothesis (2) of Proposition 6.4.2. For $\arg \varphi^{3}>$ $\pi / 2-\pi / p, \Delta_{123} \cap \Delta_{231}=\widetilde{s}_{21}=s_{31}$ and $\Delta_{123} \cap \Delta_{312}=s_{23}=\widetilde{s}_{13}$, the $C$-reflections $\left(R_{1} R_{2} R_{3}\right)^{2},\left(R_{2} R_{3} R_{1}\right)^{2}$ may not be an admissible pair (cf. $\S 2$ ). The subgroup of $\Gamma(\varphi)$ that fixes the point $s_{31}$ may not be finite. Thus hypothesis (3) of Proposition 6.4.2 is generally violated.

REMARK 2. The proof of Theorem 19.1 is so much longer than the proof of Proposition 6.4.2 because it proves the added assertion: $\Gamma$ operates holomorphically on $Y$.

REMARK 3. By a well-known theorem of Selberg (cf. [9]) any finitely generated matrix group $\Gamma$ has a subgroup of finite index $\Gamma_{0}$ that has no elements of finite order. It follows readily that if $\gamma p=$ $p, \gamma \in \Gamma_{0}$ and $p \in B$, then $\gamma=1$. A fortiori, $\Gamma_{0}$ operates freely on $Y$. Therefore $Y / \Gamma_{0}$ is a complex analytic manifold and compact for $\left|\arg \varphi^{3}\right|<\pi / 2-\pi / p$ and $(1 / \pi) \arg \varphi^{3} \in \boldsymbol{Q}$. In order to prove that $Y / \Gamma_{0}$ is an algebraic manifold, it suffices by Kodaira's fundamental theorem to construct a Kaehler metric on $Y / \Gamma_{0}$ admitting a positive line bundle. 3uch a metric can be constructed with the help of the 
Bergman kernel function for the domain $|u|^{2 m}+|v|^{2 n}<1$ in $C^{2}$. The canonical line bundle turns out to be positive (cf. [8]). In [8], it is shown that if $m=1$, the Kaehler metric can be selected so as to have everywhere negative sectional curvature.

\section{Presentation for $\Gamma$.}

Let $\varphi$ be a complex number with $|\varphi|=1, \varphi^{N}=1$, and $\left|\arg \varphi^{3}\right|<$ $\pi / 2-\pi / p$. Let $\Gamma=\Gamma(\varphi), Y=Y(\varphi)$, and $\pi: Y \rightarrow C h^{2}$ the canonical $F$ map. Let $Y^{\natural}$ denote the simply connected covering space of $Y$, let $\Gamma^{\natural}$ denote the lift of $\Gamma$ to $Y^{\natural}$, and let $\sigma: Y^{\natural} \rightarrow Y$ denote the covering map. Thus for each $\gamma^{\natural} \in \Gamma^{\natural}$ we have the commutative diagram

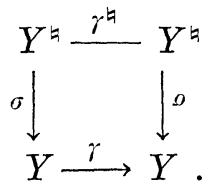

The cell decomposition $Y=\Gamma \Omega$ lifts to a cell decomposition $Y^{\natural}=$ $\Gamma^{\sharp} \Omega^{\natural}$ with $\sigma: \Omega^{\natural} \rightarrow \Omega$ a homeomorphism. By the result in $\S 18.3, Y$ has a Riemannian matrix which is preserved by $\Gamma$. The pull-back of this metric to $Y^{\natural}$ gives a metric preserved by $\Gamma^{\natural}$. The hypotheses of Theorem 6.3.2 are satisfied by $\left(\Omega^{\natural}, \Gamma^{\natural}, Y^{\natural}\right)$.

THEOREM 20.1. Let $\phi$ be a complex number of modulus 1 with $|\arg \varphi|<\pi / 2-\pi / p$. Let $\eta=\exp (\pi i / p)$. Set

$$
\begin{array}{clrl}
\rho=\operatorname{order} \bar{\eta} i \varphi^{3}, & \sigma & =\text { order } \bar{\eta} i \bar{\varphi}^{3} \\
r=\rho \text { if } 3 \nmid \rho, & s & =\sigma \text { if } 3 \nmid \sigma \\
\rho / 3 \text { if } 3 \mid \rho & & =\sigma / 3 \text { if } 3 \mid \sigma .
\end{array}
$$

Choose $\mu, \nu$ so that

$$
\begin{array}{ll}
3 \mu+1 \equiv 0 \bmod \rho & \text { if } 3 \nmid \rho \\
3 \nu+1 \equiv 0 \bmod \sigma & \text { if } 3 \nmid \sigma .
\end{array}
$$

Let $\mathscr{F}$ denote the free group with generators $\left\{R_{i} i=1,2,3\right\}$. Let $\mathscr{R}^{\prime}$ denote the normal subgroup of $\mathscr{F}$ generated by the words

$$
\left\{R_{i}^{p}, R_{i} R_{j} R_{i} R_{j}^{-1} R_{i}^{-1} R_{j}^{-1},\left(R_{1} R_{2} R_{3}\right)^{2 r},\left(R_{3} R_{2} R_{1}\right)^{2 s}, i, j=1,2,3\right\} \text {. }
$$

Set

$$
\begin{aligned}
& \rho_{i}=\left(R_{i} R_{i+1} R_{i+2}\right)^{2 \mu} R_{i} R_{i+1} \text { if } 3 \nmid \rho \\
& \sigma_{i}=\left(R_{i} R_{i-1} R_{i-2}\right)^{2 \nu} R_{i} R_{i-1} \text { if } 3 \nmid \sigma .
\end{aligned}
$$

Let $\mathscr{R}^{\prime \prime}$ denote the normal subgroup of $\mathscr{F}$ generated by the words 


$$
\begin{array}{ll}
\left\{\rho_{1}=\rho_{2}, \sigma_{1}=\sigma_{2}\right\} & \text { if } 3 \nmid \rho \sigma,\left|\arg \varphi^{3}\right|<\frac{\pi}{2}-\frac{\pi}{p} \\
\left\{\rho_{1}=\rho_{2}\right\} & \text { if } 3 \nmid \rho, 3|\sigma,| \arg \varphi^{3} \mid<\frac{\pi}{2}-\frac{\pi}{p} \\
\left\{\sigma_{1}=\sigma_{2}\right\} & \text { if } 3|\rho, 3 \nmid \sigma,| \arg \varphi^{3} \mid<\frac{\pi}{2}-\frac{\pi}{p} \\
\quad\{1\} & \text { if } 3 \mid \rho \text { and } 3|\sigma,| \arg \varphi \mid<\frac{\pi}{2}-\frac{\pi}{p} \\
\left\{\rho_{1}=\rho_{2}\right\} & \text { if } 3 \nmid \rho, \frac{\pi}{2}-\frac{\pi}{p}<\arg \varphi^{3} \\
\left\{\sigma_{1}=\sigma_{2}\right\} & \text { if } 3 \mid \rho, \arg \varphi^{3}<-\left(\frac{\pi}{2}-\frac{\pi}{p}\right) .
\end{array}
$$

Let $\Gamma$ denote the action of $\Gamma(\varphi)$ on $\mathrm{Ch}^{2}$.

(i) If, in addition $\left|\arg \varphi^{3}\right|<\pi / 2-\pi / p$, then

$$
\Gamma^{\natural}=\mathscr{F} \mid \mathscr{R}^{\prime} \cdot \mathscr{R}^{\prime \prime} \text {. }
$$

(ii) If $|\arg \varphi|<\pi / 2-\pi / p$ and $\Omega(\varphi)$ satisfies (CD1) and (CD2) (cf. $\S 6.3)$, then

$$
\Gamma=\mathscr{F} \mid \mathscr{R}^{\prime} \mathscr{R}^{\prime \prime}
$$

Proof. We first observe that the relations $\mathscr{R}^{\prime}$ and $\mathscr{R}^{\prime \prime}$ are symmetric in $1,2,3 . \mathscr{R}^{\prime}$ is symmetric since $R_{i}^{-1}\left(R_{i} R_{j} R_{k}\right)^{2} R_{i}=$ $\left(R_{j} R_{k} R_{i}\right)^{2}$ for any $i, j, k$. As for $\mathscr{R}^{\prime \prime}$, given $\rho_{1}=\rho_{2}$, we have on the one hand

$$
R_{2}^{-1} \rho_{1}=\left(R_{1} R_{2} R_{3}\right)^{2 \mu} R_{2}^{-1} R_{1} R_{2} \quad \text { (cf. Proof of Lemma } 14.1 \text { (i)). }
$$

From $R_{1} R_{2} R_{1}=R_{2} R_{1} R_{2}$, we get $R_{2}^{-1} R_{1} R_{2}=R_{1} R_{2} R_{1}^{-1}$. Hence $R_{2}^{-1} \rho_{1}=$ $\rho_{1} R_{1}^{-1}$. On the other hand

$$
R_{2}^{-1} \rho_{1}=R_{2}^{-1} \rho_{2}=\left(R_{3} R_{1} R_{2}\right)^{2} R_{3}=\rho_{3} R_{1}^{-1} .
$$

Hence $\rho_{1} R_{1}^{-1}=\rho_{3} R_{1}^{-1}$ and $\rho_{1}=\rho_{3}$. Similarly $\sigma_{1}=\sigma_{2}$ implies $\sigma_{2}=\sigma_{3}$. The relations $\mathscr{R}^{\prime} \cup \mathscr{R}^{\prime \prime}$ coincide with the relations obtained around the codimension-2 circuits of the region $\Omega(\varphi)$ by (10.1.2) and the results in $\S 17.1$ and $\S 18.4$. Theorem 20.1 now follows directly from Theorem 6.3.2.

REMARK 1. From the relations $\mathscr{R}^{\prime}$ one can infer

$$
\rho_{3} \rho_{2} \rho_{1}=1 \quad \sigma_{1} \sigma_{2} \sigma_{3}=1 .
$$

Proof. 


$$
\begin{aligned}
\rho_{3} \rho_{2} \rho_{1} & =\left(R_{3} R_{1} R_{2}\right)^{2 \mu+1} R_{2}^{-1} \cdot\left(R_{2} R_{3} R_{1}\right)^{2 \mu+1} R_{1}^{-1}\left(R_{1} R_{2} R_{3}\right)^{2 \mu+1} R_{3}^{-1} \\
& =\left(R_{3} R_{1} R_{2}\right)^{2 \mu+1}\left(R_{3} R_{1} R_{2}\right)^{2 \mu+1} R_{2}^{-1} R_{1}^{-1}\left(R_{1} R_{2} R_{3}\right)^{2 \mu+1} R_{3}^{-1} \\
& =\left(R_{3} R_{1} R_{2}\right)^{4 \mu+2+2 \mu}=\left(R_{3} R_{1} R_{2}\right)^{2(3 \mu+1)}=1 .
\end{aligned}
$$

The isomorphism $J^{\prime} V(\varphi) \rightarrow V(\bar{\varphi})$ yields $\sigma_{1} \sigma_{2} \sigma_{3}=1$. The added relation $\rho_{1}=\rho_{2}$ therefore implies $\rho_{1}^{3}=1$. The independence of $\mathscr{R}^{\prime \prime}$ from $\mathscr{R}^{\prime}$ is assured by the existence of the 3 -fold branched $\Gamma$-cover $Y^{\sharp}$ of $\S 6.5$. Similarly $\sigma_{1}=\sigma_{2}$ implies that $\sigma_{1}^{3}=1$. The group $\mathscr{F} / \mathscr{R}^{\prime}$ is the group operating on the lift of $\Gamma$ to the simply connected covering space of $Y^{\sharp}$ (which is not a manifold!).

REMARK 2. For those $\varphi$ for which $\Omega(\varphi)$ satisfies condition (CD1) and (CD2) (cf. §17.1) Theorem 20 gives a presentation for the image of the lattice subgroup $\Gamma(\varphi)$ in $\mathrm{PU}(H)$ - or equivalently, for $\Gamma(\varphi) / Z$, where $Z$ is the set of scalar multiplies of the identity matrix in the matrix group $\Gamma(\varphi)$. In general, one may possibly have a nontrivial extension

$$
1 \longrightarrow N \longrightarrow \Gamma \longrightarrow \Gamma \longrightarrow 1
$$

where $N=\pi_{1}(Y)$, the fundamental group of the space $Y(\varphi)$. I do not know whether there exist any values of $\varphi$ with $N(\varphi) \neq\{1\}$; equivalently, can $Y(\varphi)$ fail to be simply connected?

\section{Some examples of isomorphisms among $\Gamma(\varphi)$.}

Let $c_{i}=\left(R_{i} R_{i+1} R_{i+2}\right)^{2}$, regarded as an element of $\mathrm{PU}(H)$ for any integer $i \bmod 3$.

LEMMA 21.1. Let $\Gamma$ denote the group $\Gamma(\varphi)$ action on $C h^{2}$ with diagram

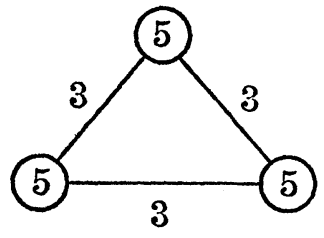

and $\varphi^{3}=\sqrt{-1}$. Then

(i) $\Gamma$ is an arithmetic lattice in $\mathrm{PU}(H)$.

(ii) $\Gamma$ is generated by $\left\{c_{1}, c_{2}, c_{3}\right\}$.

Proof of (i). Let $k=\boldsymbol{Q}[\operatorname{Tr} \mathrm{Ad} \Gamma]$. Then $k=\boldsymbol{Q}[\cos 2 \pi / 5]$ by Lemma 17.2.1. Direct calculation shows that ${ }^{\circ} \Delta>0$ for $1 \neq \sigma \in \mathrm{Gal} k$, $\Delta$ denoting the determinant of the matrix $\left\langle e_{i}, e_{j}\right\rangle(i, j=1,2,3)$. Hence $\Gamma$ is an arithmetic lattice (cf. $\S 4$ ). 
Proof of (ii). By Lemma 16.1, the order of the transformation $c_{j}$ equals the order of $\bar{\eta} i \varphi^{3}$, which is the order of $-\eta^{5}$. Thus $c_{j}$ is of order 5 for $j=1,2,3$. Inasmuch as $\arg \varphi^{3}>-(\pi / 2-\pi / 5)$, we have $\left\langle v_{123}, v_{123}\right\rangle>0$ (by $\left.(9.1 .4)^{\prime}\right)$ and $I_{i+1}=v_{i, i+1, i+2}^{\perp} \cap V^{-}$is not empty. By Lemma 13.3 (ii), $c_{i}$ fixes each point of $I_{i+1}$ and is thus a $\boldsymbol{C}$-reflection. Moreover, by Lemma 12.3

$$
I_{i} \cap I_{i+1}=t_{i, i+1} \quad(i \text { integer } \bmod 3)
$$

and thus each of $I_{1}, I_{2}, I_{3}$ meets the other two. Thus $c_{1}, c_{2}$ fix the point $t_{23}$. Direct calculation shows that for $\varphi^{3}=i$,

$$
\begin{aligned}
\frac{\left\langle v_{123}, v_{231}\right\rangle}{\left|v_{123}\right|\left|v_{231}\right|}= & -\alpha \varphi \frac{\left(1-\eta-2 \bar{\eta}^{2}\right)}{1+\eta+\bar{\eta}^{2}}=-\alpha \varphi \bar{\eta}^{2} \\
& \left(R_{3} R_{1} R_{2}\right)^{-2} t_{23}=p_{12} .
\end{aligned}
$$

The first equation implies that $\left\{\left\{c_{1}, c_{2}\right\}\right\} \approx F_{12}$. The second equation states that the stabilizer $\Gamma_{p_{12}}$ of the point $p_{12}$ contains $\left\{c_{3}^{-1} c_{1} c_{3}\right.$, $\left.c_{3}^{-1} c_{2} c_{3}\right\}$. Since $\Gamma$ is a discrete group, $\Gamma_{p_{12}}$ is a finite group. Since (5) 3 (5) is a maximal subgroup of PU (2) generated by $C$-reflections, it follows that $\left\{\left\{c_{3}^{-1} c_{1} c_{3}, c_{3}^{-1} c_{2} c_{3}\right\}\right\}=\Gamma_{12}$. Hence $\left\{\left\{c_{1}, c_{2}, c_{3}\right\}\right\} \supset\left\{R_{1}\right.$, $\left.R_{2}\right\}$. By symmetry $\left\{\left\{c_{1}, c_{2}, c_{3}\right\}\right\} \supset\left\{R_{1}, R_{2}, R_{3}\right\}$. This implies (ii).

EXAMPLE 1. Equation (21.1) has an interesting interpretation. $\left(\varphi \bar{\eta}^{2}\right)^{3}=\phi^{3} \bar{\eta}^{6}=-\bar{\eta} i$. Thus there is an isomorphism $A$ of $\Gamma$ to $\Gamma(\psi)$ with arg $\psi^{3}=-7 \pi / 10$ given by

A:

$$
c_{i}(\varphi) \longrightarrow R_{i}^{2}(\psi) \quad(i=1,2,3) .
$$

The reason for not sending $c_{1}$ to $R_{1}$ is that $c_{1}$ rotates about its fixed point set via $\bar{\eta} i \varphi^{3}$ by (9.1.1), i.e., by $-\bar{\eta}$. Thus $c_{1}^{3}$ should map to $R_{1}(\psi)$. The isomorphism $A$ is induced by the automorphism of $C^{3}$

$$
v_{i, i+1, i+2} \longrightarrow e_{i} \quad(i \text { integer } \bmod 3) .
$$

An alternative description is to say that $A$ is induced by the isometry of the $\boldsymbol{C} h^{2}$ formed from $V(\varphi)$ to the $\boldsymbol{C} h^{2}$ formed from $V(\psi)$ which takes

$$
t_{23} \longrightarrow p_{12}, t_{31} \longrightarrow p_{23}, t_{12} \longrightarrow p_{23} \text {. }
$$

If we compose $A$ with $J^{\prime}: V(\psi) \rightarrow V(\psi)$, we get that $J^{\prime} \circ A$ induces

$$
t_{12} \longrightarrow p_{12}, t_{23} \longrightarrow p_{23}, t_{31} \longrightarrow p_{31} \text {. }
$$

This example provides us with a fundamental domain for the arithmetic lattice $\Gamma(\varphi)$. For $\Omega(\varphi)$ does not satisfy condition (CD2) 
and is therefore not a fundamental domain $\bmod \mathrm{Aut}_{\Gamma} \Omega$. However, $\Omega(\psi)$ is a fundamental domain $\bmod \operatorname{Aut}_{\Gamma} \Omega(\psi)\left(=\left\{1, J, J^{2}\right\}\right)$.

The next example will provide a geometric isomorphism between two arithmetic lattices generated by $\boldsymbol{C}$-reflections having different Coxeter diagrams.

EXAMPLE 2. Let $\Gamma^{\prime}$ denote the group generated by reflections with diagram

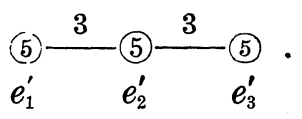

Inasmuch as there are no closed loops in the diagram, the phase shifts can be arbitrary. Here $\left\{\left\{R_{1}^{\prime}, R_{2}^{\prime}\right\}\right\}$ and $\left\{\left\{R_{2}^{\prime}, R_{3}^{\prime}\right\}\right\}$ are isomorphic groups of $\Gamma^{\prime}$ where as $R_{1}^{\prime} R_{3}^{\prime}=R_{3}^{\prime} R_{1}^{\prime}$. It is easily verified that $\Gamma^{\prime}$ is discrete by the arithmeticity test of $\S 4$. Let $\varphi=\exp \pi i / 6$ as in Example 1. Then there is an isomorphism of $\Gamma^{\prime}$ to $\Gamma(\varphi)$ given by

$\mathrm{A}^{\prime}$ :

$$
\begin{aligned}
& R_{1}^{\prime} \longrightarrow R_{1}(\varphi) \\
& R_{2}^{\prime} \longrightarrow R_{2}(\varphi) \\
& R_{3}^{\prime} \longrightarrow\left(R_{3} R_{1} R_{2}\right)^{6}(\varphi) .
\end{aligned}
$$

Let $p_{i j}^{\prime}=e_{i}^{\perp} \cap e_{j}^{\perp}(i, j=1,2,3, i \neq j)$. The isomorphism $\mathrm{A}^{\prime}$ is induced by the isometry of $C h^{2}$ which takes

$$
p_{13}^{\prime} \longrightarrow t_{32}(\varphi), p_{12}^{\prime} \longrightarrow p_{12}(\varphi), p_{23}^{\prime} \longrightarrow t_{31}(\varphi) \text {. }
$$

It should be noted that the image of $\mathrm{A}^{\prime}$ is all of $\Gamma(\varphi)$ since it contains $\left(R_{3} R_{1} R_{2}\right)^{2},\left(R_{2} R_{3} R_{1}\right)^{2}=R_{2}\left(R_{3} R_{1} R_{2}\right)^{2} R_{2}^{-1}$, and $\left(R_{1} R_{2} R_{3}\right)^{2}$.

\section{Nonstandard homomorphisms.}

Given a nonarithmetic lattice $\Gamma$ in $\mathrm{PU}(2,1)$, there is a field automorphism $\sigma$ of $C$ such that ${ }^{\sigma} \Gamma$ is not a bounded set of matrices and $\sigma$ is nontrivial on the field $Q[\operatorname{Tr} A d \Gamma]$ (cf. Lemma 4.1). Such a monomorphism $\sigma$ of $\Gamma$ cannot be extended to a rational representation of $\mathrm{PU}(2,1)$ - for such a representation $\rho$ would satisfy $\operatorname{Tr} \operatorname{Ad} \rho(\gamma)=$ $\operatorname{Tr} \operatorname{Ad} \gamma$ for all $\gamma \in \Gamma$. This is in sharp contrast to the remarkable "super-rigidity" theorem proved by Margulis for semi-simple groups of $\boldsymbol{R}$-rank $>1$.

There is however, another kind of violation of super-rigidity: There is a homomorphism $\rho$ of $\Gamma\left(\varphi_{1}\right)$ onto $\Gamma\left(\phi_{2}\right)$ which is not a composition of a rational homomorphism and a field automorphism; this will follow once we show that $\operatorname{Ker} \rho$ is infinite. Moreover, in our example $\Gamma\left(\varphi_{1}\right)$ and $\Gamma\left(\varphi_{2}\right)$ are arithmetic lattices. 
Let $\Gamma_{1}=\Gamma\left(\varphi_{1}\right)$ and $\Gamma_{2}=\Gamma\left(\varphi_{2}\right)$ be the groups generated by $C$ reflections with Coxeter diagrams

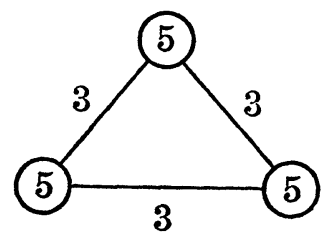

with $\arg \varphi_{1}^{3}=-\pi / 10, \arg \varphi_{2}^{3}=7 \pi / 10$. By Theorem 20.1, we know that the relations in the presentation for $\Gamma_{1}$ and $\Gamma_{2}$ are $\left\{R_{i}^{5}=1\right.$, $\left.R_{i} R_{j} R_{i}=R_{j} R_{i} R_{j} i, j=1,2,3\right\}$ and in addition for $\Gamma_{1}$

$$
\left\{\begin{array}{l}
\left(R_{1} R_{2} R_{3}\right)^{20}=1,\left(R_{3} R_{2} R_{1}\right)^{10}=1 \\
\left(R_{1} R_{2} R_{3}\right)^{6} R_{1} R_{2}=\left(R_{2} R_{3} R_{1}\right)^{6} R_{2} R_{3}=\left(\left(R_{3} R_{2} R_{1}\right)^{6} R_{3} R_{2}\right)^{-1}
\end{array}\right.
$$

and for $\Gamma_{2}$

$$
\left\{\begin{array}{l}
\left(R_{1} R_{2} R_{3}\right)^{4}=1 \\
\left(R_{1} R_{2} R_{3}\right)^{2} R_{1} R_{2}=\left(R_{2} R_{3} R_{1}\right)^{2} R_{2} R_{3}
\end{array}\right.
$$

Moreover, in $\Gamma_{2}$ we have $\left(\left(R_{3} R_{2} R_{1}\right)^{6} R_{3} R_{2}\right)^{-1}=\left(R_{1} R_{2} R_{3}\right)^{6} R_{1} R_{2}$, by Lemma 16.1 and $\left(R_{3} R_{2} R_{1}\right)^{10}=1$ by (9.1.2). Thus the map $\rho: R_{i}\left(\varphi_{1}\right) \rightarrow R_{i}\left(\varphi_{2}\right)$ $(1=1,2,3)$ is a homomorphism. Its kernel is a normal subgroup $N$ of $\Gamma\left(\varphi_{1}\right)$ containing $\left(R_{1} R_{2} R_{3}\right)^{4},\left(R_{2} R_{2} R_{1}\right)^{4},\left(R_{3} R_{1} R_{2}\right)^{4}$, whose common fixed point set is $I_{1} \cap I_{2} \cap I_{3}$ which is empty. Since every finite subgroup of $\Gamma_{1}$ fixes a point in the ball, $N$ is not finite. Consequently $\rho$ is not a composition of the stated type. Consulting Table 3 of $\S 17.3$, we see that both $\Gamma\left(\varphi_{1}\right)$ and $\Gamma\left(\varphi_{2}\right)$ are arithmetic lattices. Both these lattices are cocompact.

\section{REFERENCES}

1. A. Borel, Density properties for certain subgroups of semi-simple groups without compact components, Ann. of Math., 72 (1960), 179-188.

2. A. Borel and Harish-Chandra, Arithmetic subgroups of algebraic groups, Ann. of Math., 75 (1962), 485-535.

3. A. Cohen, Finite complex reflection groups, Ann. Scient. Ec. Norm. Sup., 9 (1976), $379-436$.

4. H. S. M. Coxeter, Finite groups generated by unitary reflections, Abh. Math. Sem. Univ. Hamburg, 31 (1967), 125-135.

5. G. P. Hochschild and G. D. Mostow, Representations and representative functions on Lie groups, Ann. of Math., 66 (1957), 495-542.

6. G. D. Mostow, Strong rigidity of locally symmetric spaces, Ann. of Math. Studies. 78 (1973), 1-195.

7. - Existence of a non-arithmetic lattice in $S U(2,1)$, Proc. Nat. Acad. Sci., 75, 3029-3033.

8. G. D. Mostow, and Y. T. Siu, A compact Kaehler surface of negative curvature not covered by the ball, to appear in Annals of Math. 
9. A. Selberg, On discontinuous groups in higher dimensional symmetric spaces, International Colloquium on Function Theory, Tata Inst. of Fund. Research (Bombay) (1960), 147-164.

10. G. C. Shepherd and J. A. Todd, Finite unitary reflection groups, Canad. J. Math., 6 (1954), 274-304.

Received August 3, 1979. Supported in part by N. S. F. Grant \#MCS76-10435.

YALE UNIVERSITY

New Haven, CT 06520 


\section{PACIFIC JOURNAL OF MATHEMATICS}

\section{EDITORS}

DONALD BABBITT (Managing Editor)

University of Galifornia

Los Angeles, California 90024

HUGo RossI

University of Utah

Salt Lake City, UT 84112

C. C. MOORE AND ANDREW OGG

University of California

Berkeley, CA 94720
J. DUGUNDJI

Department of Mathematics University of Southern California Los Angeles, California 90007

R. FINN AND J. MILGRAM Stanford University Stanford, California 94305

\section{ASSOCIATE EDITORS}

E. F. BECKENBACH

B. H. NEUMANN

F. WOLF

K. YosHIDA

\section{SUPPORTING INSTITUTIONS}

UNIVERSITY OF BRITISH COLUMBIA CALIFORNIA INSTITUTE OF TECHNOLOGY UNIVERSITY OF CALIFORNIA MONTANA STATE UNIVERSITY UNIVERSITY OF NEVADA, RENO NEW MEXICO STATE UNIVERSITY OREGON STATE UNIVERSITY UNIVERSITY OF OREGON
UNIVERSITY OF SOUTHERN CALIFONIA STANFORD UNIVERSITY UNIVERSITY OF HAWAII UNIVERSITY OF TOKYO UNIVERSITY OF UTAH WASHINGTON STATE UNIVERSITY UNIVERSITY OF WASHINGTON 


\section{Pacific Journal of Mathematics}

\section{Vol. 86, No. $1 \quad$ November, 1980}

Gert Einar Torsten Almkvist, Invariants, mostly old ones .............. 1

Hyman Bass, Groups of integral representation type ................ 15

A. Białynicki-Birula, On action of SL(2) on complete algebraic

varieties........................................

Frederick Paul Greenleaf and Martin Allen Moskowitz, Groups of

automorphisms of Lie groups: density properties, bounded orbits, and

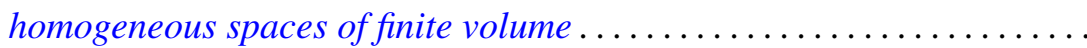

Raymond Taylor Hoobler, A cohomological interpretation of Brauer groups

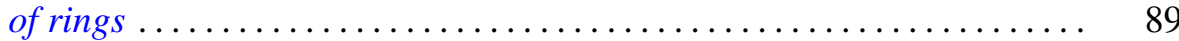

Irving Kaplansky, Superalgebras ........................ 93

Jerrold Lewis Kleinstein and Alex I. Rosenberg, Succinct and

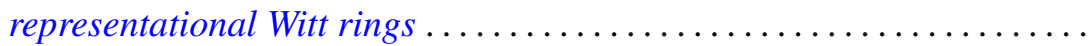

E. R. Kolchin, On universal extensions of differential fields ............ 139

Andy R. Magid, Analytic subgroups of affine algebraic groups. II ....... 145

Calvin Cooper Moore, The Mautner phenomenon for general unitary

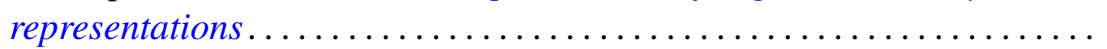

George Daniel Mostow, On a remarkable class of polyhedra in complex hyperbolic space ................................ 171

Brian Lee Peterson, Extensions of pro-affine algebraic groups. II . . . . . . 277

John Henry Reinoehl, Lie algebras and affine algebraic groups......... 287

Maxwell Alexander Rosenlicht, Differential valuations .

John Brendan Sullivan, The second Lie algebra cohomology group and Weyl modules..................................... 321

Moss Eisenberg Sweedler, Right derivations and right differential

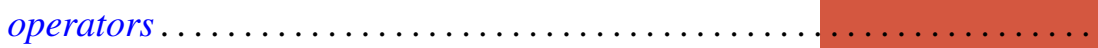

Bostwick Frampton Wyman, Time varying linear discrete-time systems. II. Duality.................................. 